Nevada

Environmental

Restoration

Project

\title{
Closure Report for Corrective Action Unit 115: Area 25 Test Cell A Facility, Nevada Test Site, Nevada
}

Controlled Copy No.:

Revision: 0

March 2006

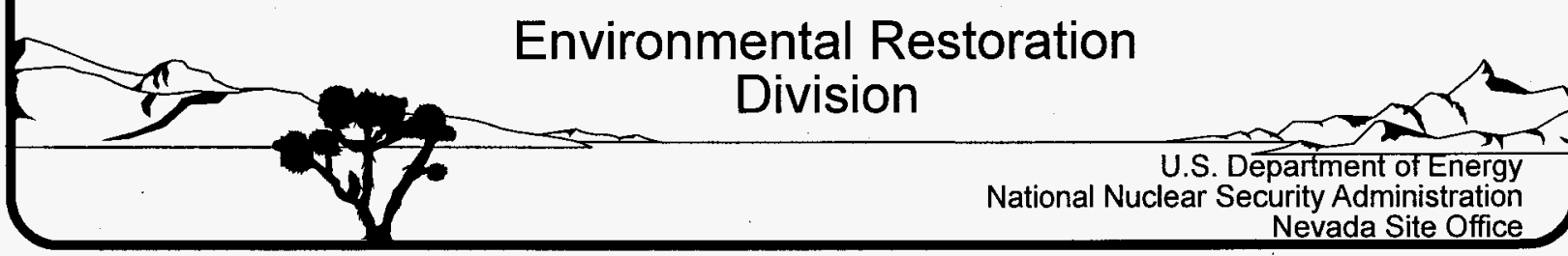




\section{DISCLAIMER}

Reference herein to any specific commercial product, process, or service by trade name, trademark, manufacturer, or otherwise, does not necessarily constitute or imply its endorsement, recommendation, or favoring by the United States Government or any agency thereof or its contractors or subcontractors.

This report has been reproduced directly from the best available copy.

Available for sale to the public from:

U.S. Department of Commerce

National Technical Information Service

5285 Port Royal Road

Springfield, VA 22161-0002

Telephone: (800) 553-6847

Fax: (703) 605-6900

E-mail: orders@ntis.gov

Online ordering: http://www.ntis.gov/ordering.htm

Available electronically at http://www.osti.gov/bridge.

Available for a processing fee to the U.S. Department of Energy and its contractors, in paper, from:

U.S. Department of Energy

Office of Scientific and Technical Information

P.O. Box 62

Oak Ridge, TN 37831-0062

Telephone: (865) 576-8401

Fax: (865) 576-5728

E-mail: reports@adonis.osti.gov 


\title{
CLOSURE REPORT FOR CORRECTIVE ACTION UNIT 115: AREA 25 TEST CELL A FACILITY, NEVADA TEST SITE, NEVADA
}

\author{
U.S. Department of Energy \\ National Nuclear Security Administration \\ Nevada Site Office \\ Las Vegas, Nevada
}

Controlled Copy No.

Revision: 0

March 2006 
THIS PAGE INTENTIONALLY LEFT BLANK 


\section{CLOSURE REPORT FOR CORRECTIVE ACTION UNIT 115: AREA 25 TEST CELL A FACILITY, NEVADA TEST SITE, NEVADA}

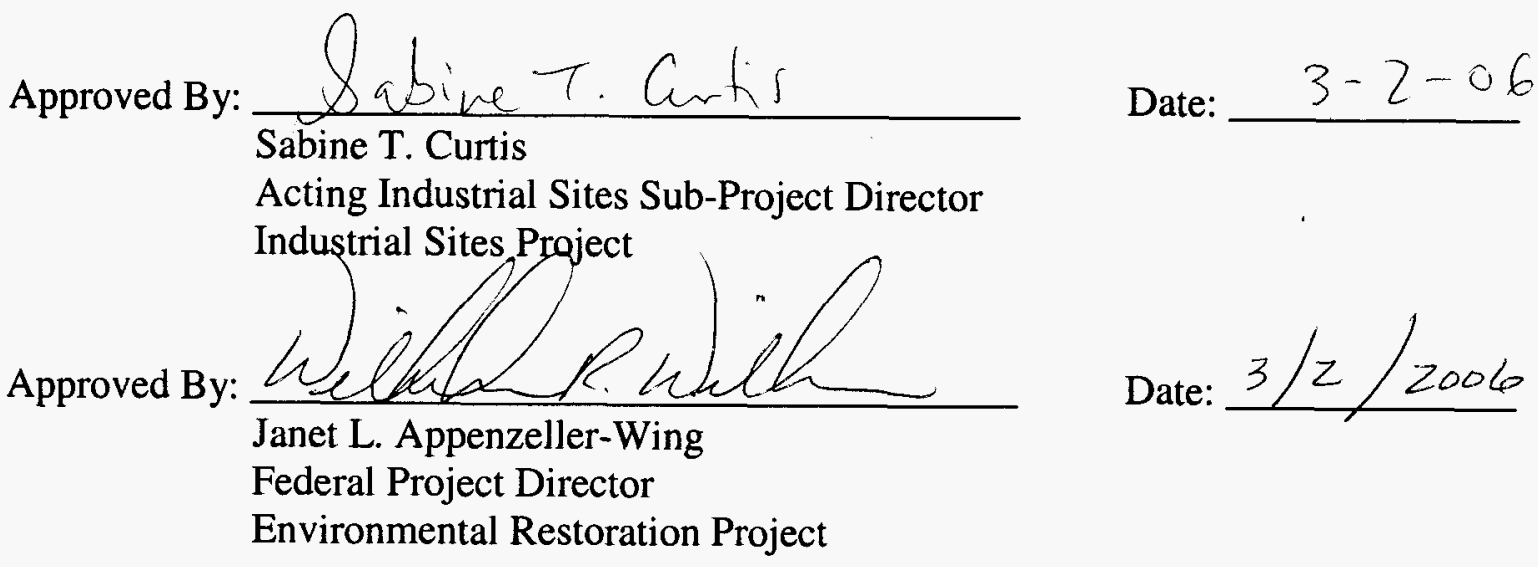


THIS PAGE INTENTIONALLY LEFT BLANK 


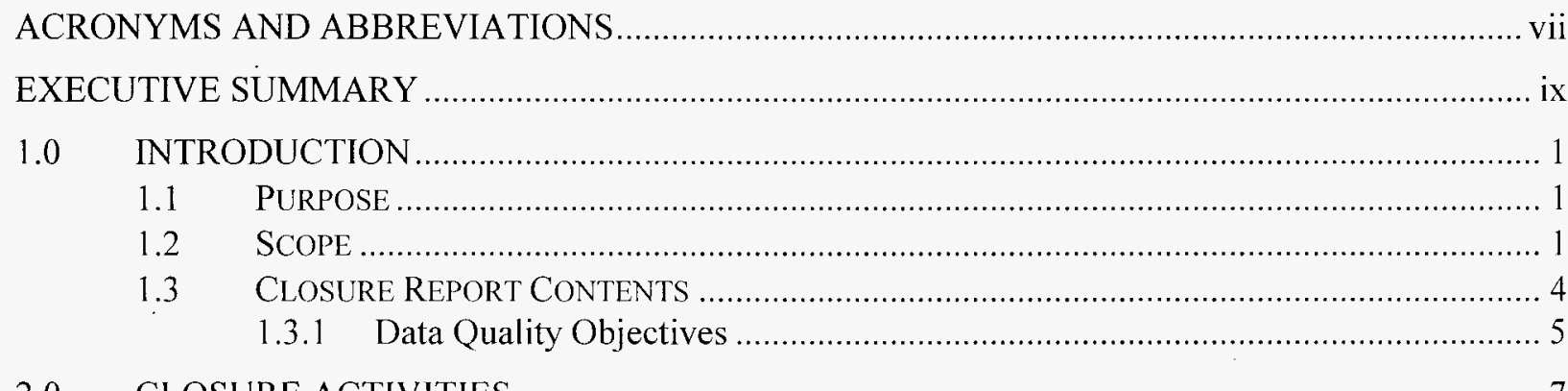

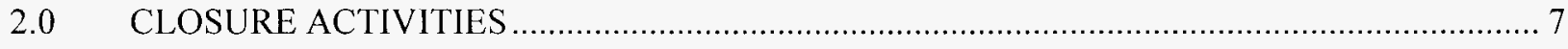

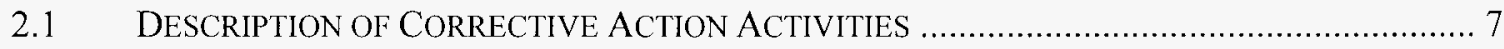

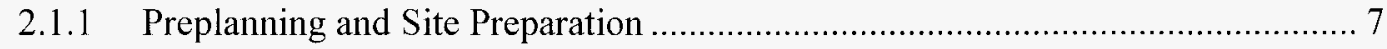

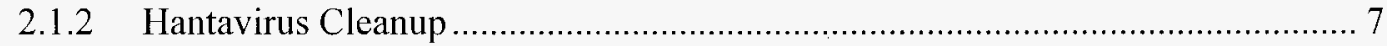

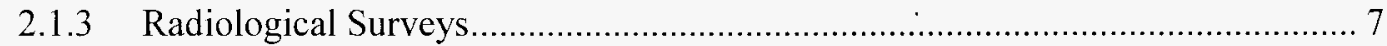

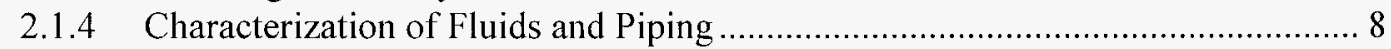

2.1.5 Removal of Hazardous/Regulated Waste.................................................... 8

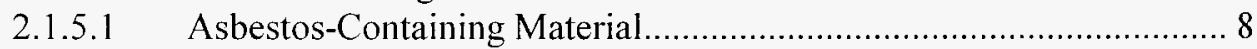

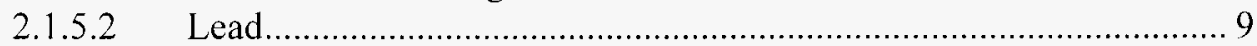

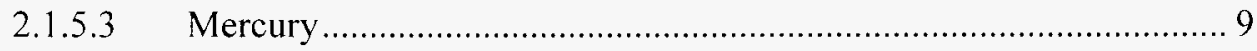

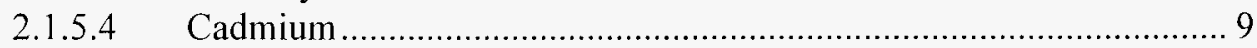

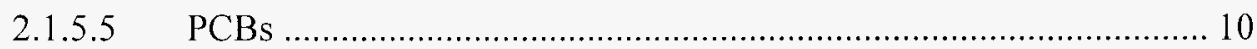

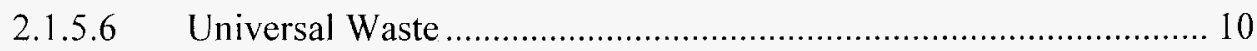

2.1.6 Removal of Radiologically Impacted Soil ................................................. 10

2.1.7 Characterization and Decontamination of Radiologically Impacted Surfaces .... 10

2.1.8 Demolition of Aboveground Structures ........................................................ 11

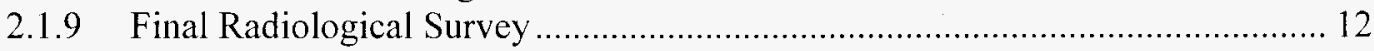

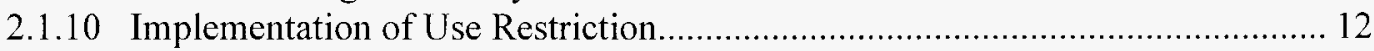

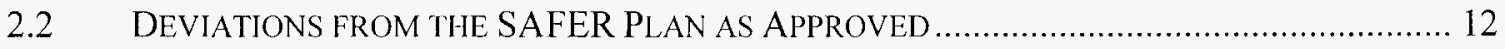

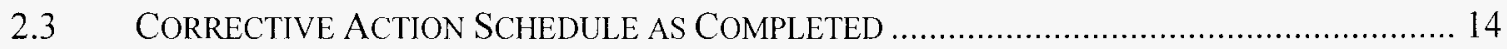

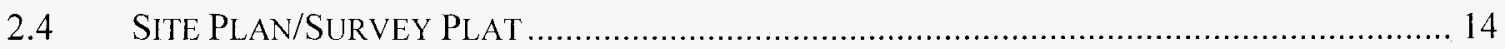

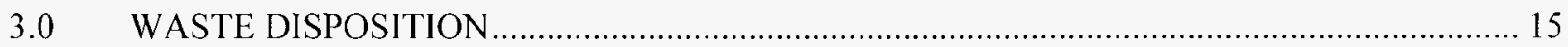

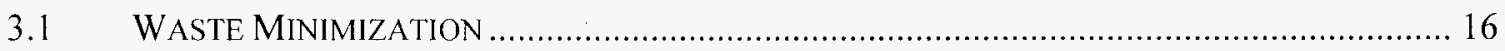

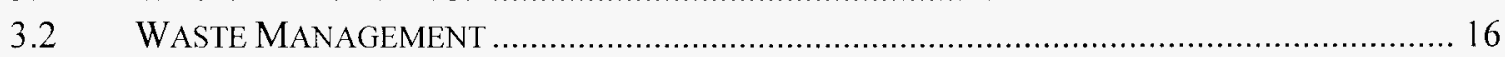

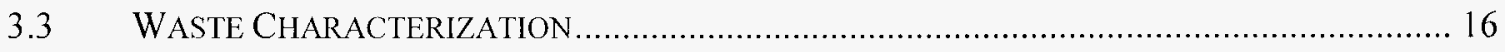

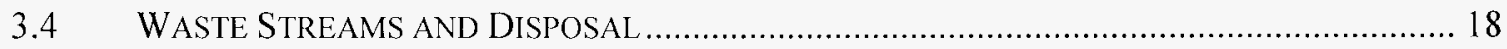

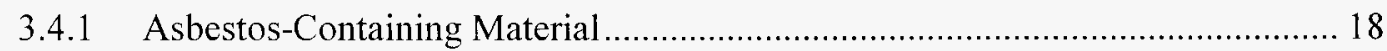

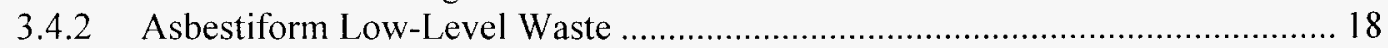

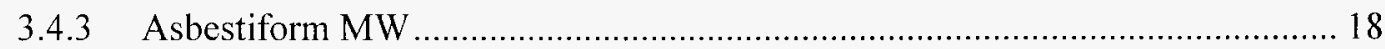

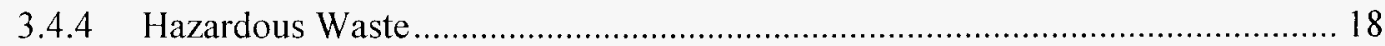

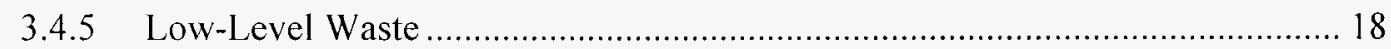

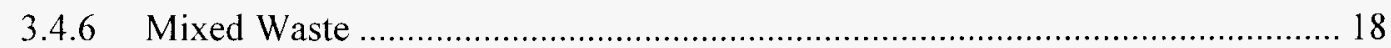

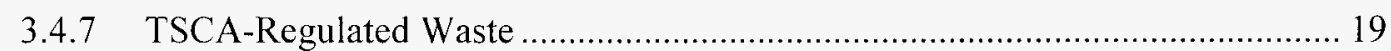

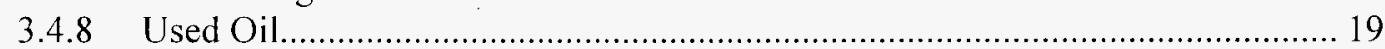

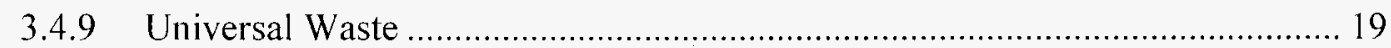

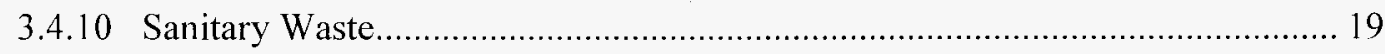




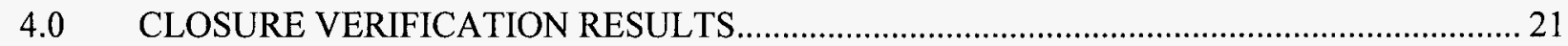

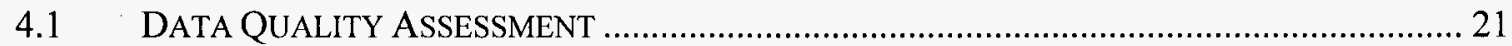

4.1.1 Quality Assurance/Quality Control Procedures ................................................ 21

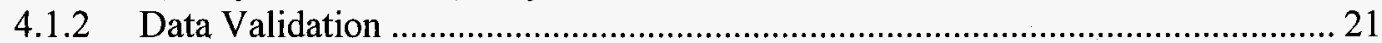

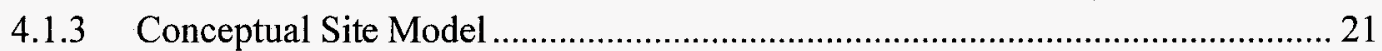

4.2 USE RESTRICTION................................................................................................. 22

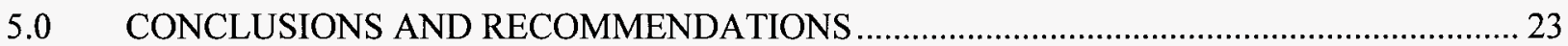

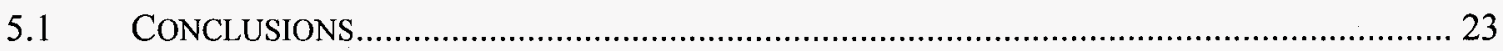

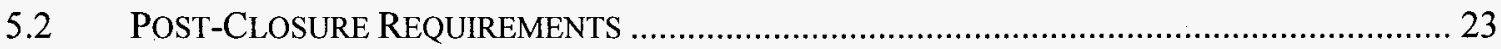

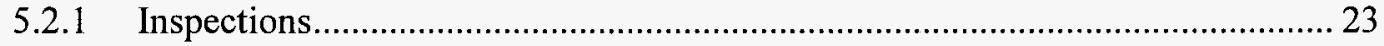

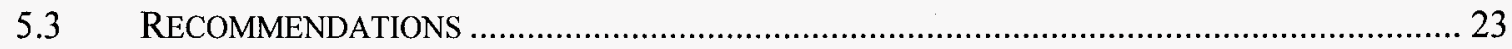

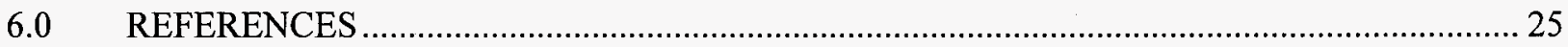

\section{FIGURES}

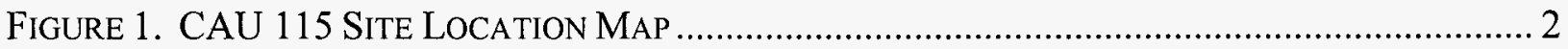

FigURE 2. CAU 115 BoundaRY AND FACILITY LAYOUT ......................................................... 3

FIGURE 3. CAU 115 USE RESTRICTION BOUNDARY ........................................................ 13

\section{TABLES}

Table 1. CAU 115 Use-Restricted Area, UTM, Zone 11, NAD 27, METERS ........................ 12

TABle 2. CAU 115 Closure Activities SCHEdULE ............................................................ 14

TABLE 3. CAU 115 WASTE Disposition SUMMARY …........................................................... 15

TABlE 4. CAU 115 WASTE Characterization SAMPLE RESUltS.......................................... 17

\section{APPENDICES}

APPENDIX A - DATA QUALITY OBJECTIVES

APPENDIX B - SAMPLE ANALYTICAL RESUlts

APPENDiX C - Final RADIOLOGICAL SURVEY RESUlts

APPENDIX D - WASTE DISPOSITION DOCUMENTATION

APPENDIX E - USE RESTRICTION DOCUMENTATION

APPENDiX F - Site Closure Photographs

APPENDIX G - RECORD OF TECHNICAL CHANGE TO THE SAFER PLAN

LIBRARY DISTRIBUTION LIST 


\section{ACRONYMS AND ABBREVIATIONS}

ACM asbestos-containing material

ALLW asbestiform low-level waste

BMP best management practice

BN Bechtel Nevada

CA contaminated area

CAS(s) Corrective Action Site(s)

CAU Corrective Action Unit

COC(s) contaminant(s) of concern

CR Closure Report

CSM conceptual site model

DOE U.S. Department of Energy

DOE/NV U.S. Department of Energy, Nevada Operations Office (used prior to April 2001)

EPA U.S. Environmental Protection Agency

ER Environmental Restoration

FFACO Federal Facility Agreement and Consent Order

$\mathrm{ft} \quad$ foot (feet)

$\mathrm{ft}^{3} \quad$ cubic foot (feet)

FY fiscal year

HEPA high-efficiency particulate air

HW hazardous waste

ISOCS In-Situ Object Counting System

LLW low-level waste

$\mathrm{mg} / \mathrm{kg} \quad$ milligram(s) per kilogram

$\mathrm{mg} / \mathrm{L} \quad$ milligram(s) per liter

MW mixed waste

NDEP Nevada Division of Environmental Protection

NNSA/NSO U.S. Department of Energy, National Nuclear Security Administration Nevada Site Office

NNSA/NV U.S. Department of Energy, National Nuclear Security Administration Nevada Operations Office

NTS Nevada Test Site 


\section{ACRONYMS AND ABBREVIATIONS (continued)}

NV/YMP Nevada/Yucca Mountain Project

PCBs polychlorinated biphenyls

QA quality assurance

QAPP Industrial Sites Quality Assurance Project Plan

QC quality control

RCRA Resource Conservation and Recovery Act

RMA(s) Radioactive Materials Area(s)

ROTC Record of Technical Change

RWMC Radioactive Waste Management Complex

SAFER Streamlined Approach for Environmental Restoration

SDG Sample Delivery Group

SVOC(s) semi-volatile organic compound(s)

TCLP Toxicity Characterization Leaching Procedure

TSCA Toxic Substances Control Act

URMA Underground Radioactive Materials Area

UW universal waste

VOC(s) volatile organic compound(s)

WMA(s) waste management area(s)

XRF $\quad x$-ray fluorescence 
The Area 25 Test Cell A Facility is identified in the Federal Facility Agreement and Consent Order (FFACO) of 1996 as Corrective Action Unit (CAU) 115. CAU 115 is located on F Road in Area 25 of the Nevada Test Site, approximately 87 miles northwest of Las Vegas, Nevada, and consists of the following four Corrective Action Sites (CASs):

- CAS 25-41-04, Test Cell A Facility

- CAS 25-99-04, Asbestos Wrapped Pipes

- CAS 25-99-05, Asbestos Wrapped Pipes

- CAS 25-99-06, Asbestos Wrapped Pipes

CAU 115 closure activities were conducted from September 2004 to September 2005 according to the FFACO and the Nevada Division of Environmental Protection-approved Streamlined Approach for Environmental Restoration (SAFER) Plan for CAU 115 (U.S. Department of Energy, National Nuclear Security Administration Nevada Site Office, 2004). The approved corrective action alternative was clean closure. However, because some of the concrete was found to be radiologically activated and could not be decontaminated to meet free release levels, the site was closed in place and a use restriction was implemented under an approved Record of Technical Change to the SAFER Plan, which is included as Appendix G of this report.

Site characterization data and process knowledge indicated that significant surface areas were radiologically contaminated above release limits and that regulated and/or hazardous wastes were present in the facility. The closure activities included:

- Removal of all hazardous and regulated wastes

- Decontamination, demolition, and disposal of Buildings 3113,3113A, and 3113B and related equipment and structures

- Characterization and partial decontamination of the concrete reactor pad and impacted concrete surfaces

- Removal of the dewar as a best management practice

- Performance of final radiological survey to establish appropriate radiological controls and document final site conditions

- Posting of radiological warning and use restriction signs

Closure activities generated waste streams consisting of asbestos-containing material, asbestiform low-level waste, asbestiform mixed waste, non-hazardous sanitary waste, hazardous waste, low-level waste, mixed waste, Toxic Substances Control Act regulated waste, used oil, and universal waste. Waste minimization activities included segregation of waste streams, recycling, and size reduction. Some wastes exceeded land disposal restriction limits and required offsite treatment prior to disposal. Other wastes meeting land disposal restrictions were disposed of in appropriate onsite or offsite landfills. Waste disposition documentation is included as Appendix D of this report. 
Extensive final radiological verification surveys were performed to determine the radiological condition of the remaining concrete slab. The survey results are presented in Appendix $\mathrm{C}$ of this report, and use restriction documentation is included as Appendix $\mathrm{E}$ of this report. The proposed post-closure requirements consist of annual site inspections to determine the condition of fencing and postings. The post-closure plan is presented in detail in Section 5.2. 
This Closure Report (CR) documents the closure activities for Corrective Action Unit (CAU) 115, Area 25 Test Cell A Facility, according to the Federal Facility Agreement and Consent Order (FFACO) of 1996 and the Nevada Division of Environmental Protection (NDEP)-approved Streamlined Approach for Environmental Restoration (SAFER) Plan (U.S. Department of Energy, National Nuclear Security Administration Nevada Site Office [NNSA/NSO], 2004). CAU 115 is located on F Road in Area 25 of the Nevada Test Site (NTS), approximately 87 miles northwest of Las Vegas, Nevada (Figure 1). CAU 115 consists of the following four Corrective Action Sites (CASs):

- CAS 25-41-04, Test Cell A Facility

- CAS 25-99-04, Asbestos Wrapped Pipes

- CAS 25-99-05, Asbestos Wrapped Pipes

- CAS 25-99-06, Asbestos Wrapped Pipes

Figure 2 shows the boundary of the CAU and the general layout of the facility. CAS 25-41-04 included Buildings 3113, 3113A, 3113B and related equipment, but did not include any piping or soil below the building foundations. CAS 25-99-04 included piping located south of Building 3113A and west of the concrete reactor pad. CAS 25-99-05 included piping within Building 3113A. CAS 25-99-06 included piping on the roof of the facility.

\subsection{PURPOSE}

This CR describes the activities performed to close CAU 115, Area 25 Test Cell A Facility, as presented in the NDEP-approved SAFER Plan (NNSA/NSO, 2004). The SAFER Plan includes a summary of the site history, process knowledge, and closure standards. This CR provides a summary of the completed closure activities, documentation of waste disposal, and analytical and radiological data to confirm that the remediation goals were met and to document final site conditions.

The approved closure alternative as presented in the SAFER Plan for CAU 115 (NNSANSO, 2004) was clean closure; however, closure in place was implemented under a Record of Technical Change (ROTC) to the SAFER Plan when radiological surveys indicated that the concrete reactor pad was radiologically activated and could not be decontaminated to meet free release levels. The ROTC is included as Appendix G of this report.

\subsection{SCOPE}

The objectives of closure were to remove any trapped residual liquids and gases, dispose regulated and hazardous waste, decontaminate removable radiological contamination, demolish and dispose aboveground structures, remove the dewar as a best management practice (BMP), and characterize and restrict access to all remaining radiological contamination. Radiological contaminants of concern (COCs) included cobalt-60, cesium-137, strontium-90, uranium234/235/236/238, and plutonium-239/240. Additional COCs included Resource Conservation and Recovery Act (RCRA) metals, polychlorinated biphenyls (PCBs), and asbestos. 


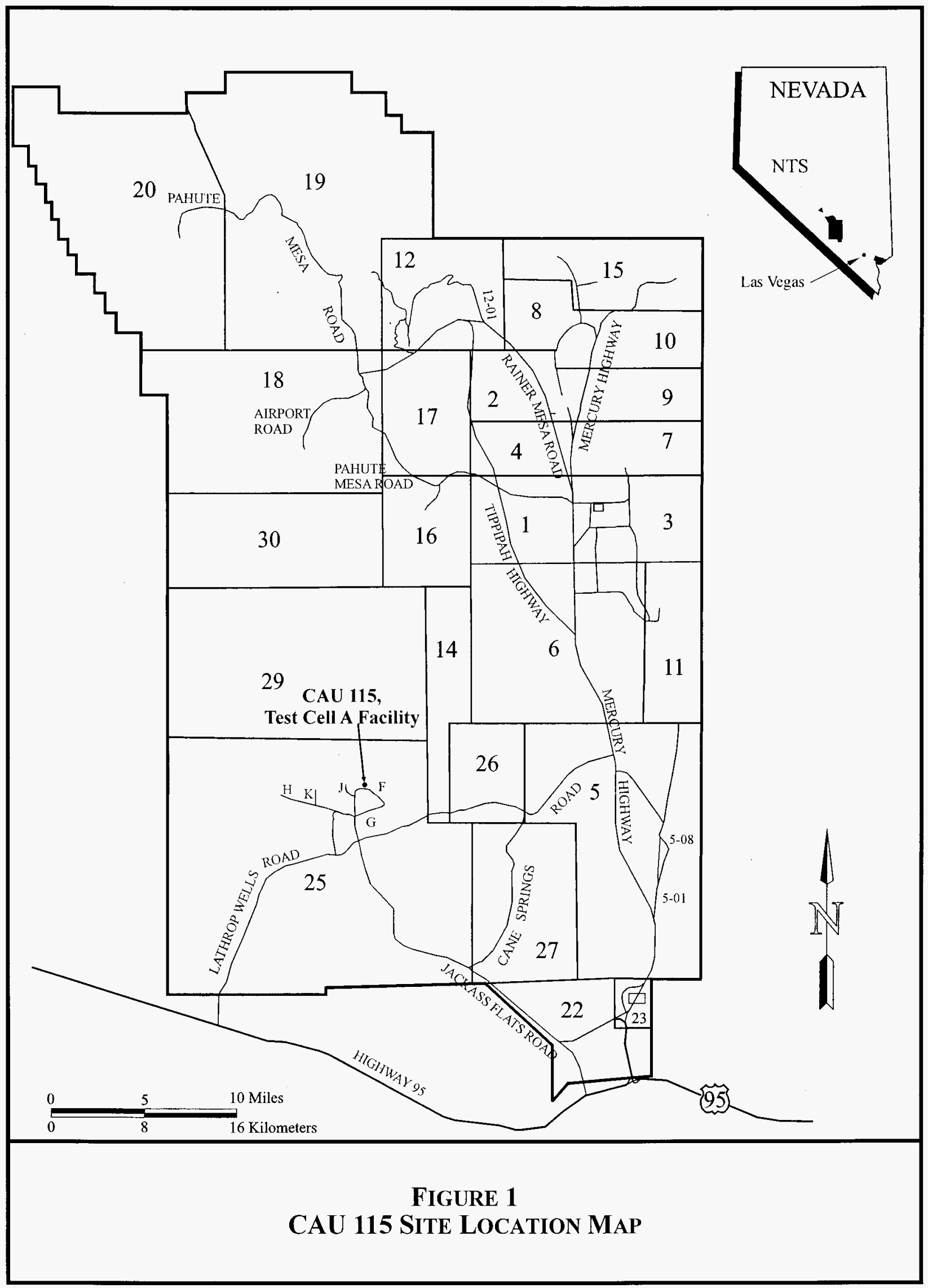




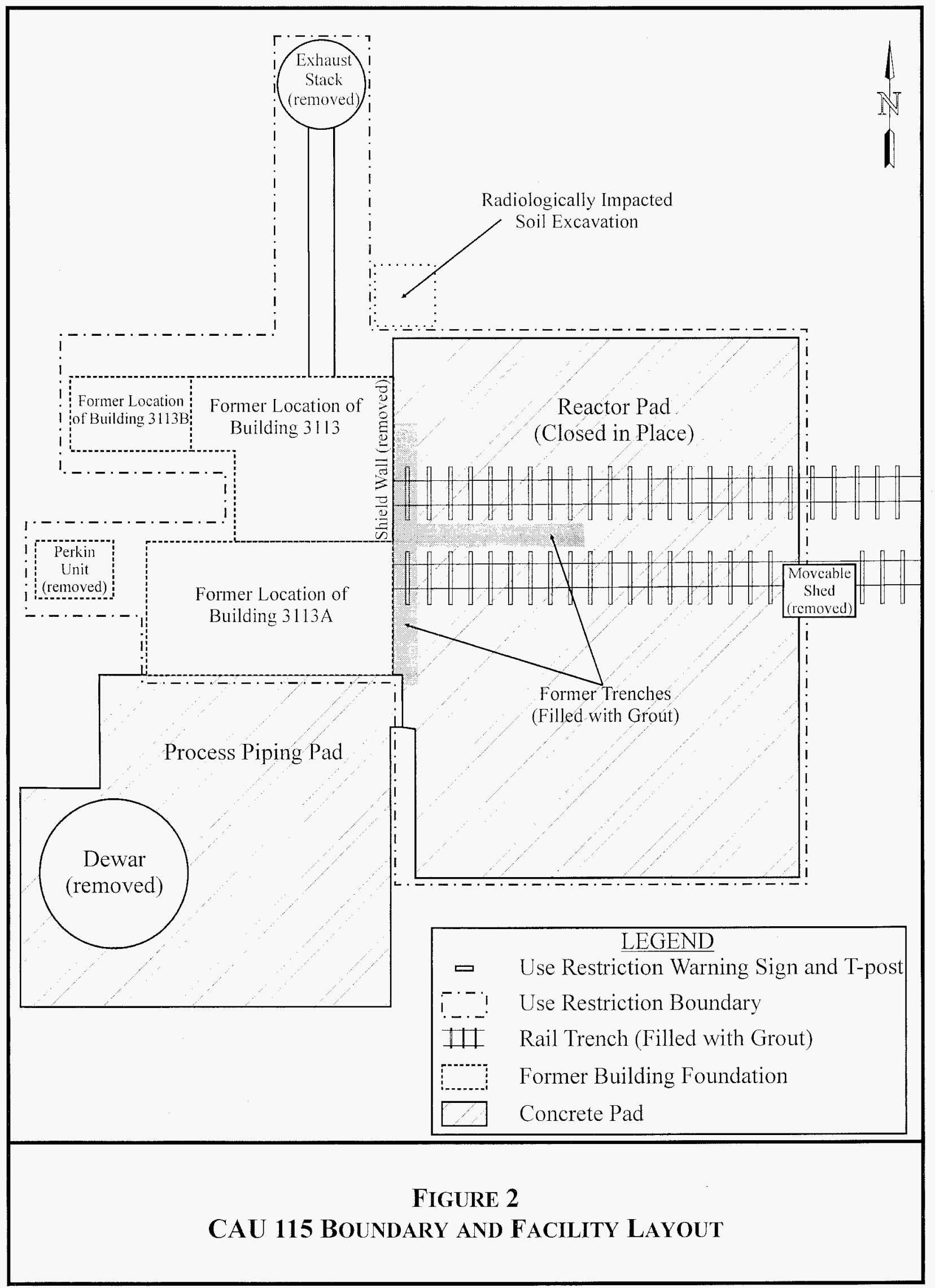


The scope of work included the following activities:

- Mitigation of safety hazards

- Removal of trapped residual liquids and gases

- Removal of all hazardous and regulated wastes

- Asbestos-containing material (ACM) (pipe/conduit insulation, roof mastic sealant)

- Lead (bricks, wool)

- Mercury (circuit boards)

- Cadmium (foil covering insulated pipe/conduit)

- PCBs (fluorescent light ballasts, paint)

- Decontamination, demolition, and disposal of Buildings 3113, 3113A, and 3113B and related equipment and structures

- Characterization and decontamination of removable radiological contamination on the reactor concrete pad and building foundations

- Removal of the dewar as a BMP

- Performance of final radiological survey to establish appropriate radiological controls and document final site conditions

- Posting of radiological and use restriction warning signs

- Appropriate disposal of all generated waste

Clean closure and free release of the site, as originally planned, was not possible due to radiological activation of the concrete near the former location of the reactor. Soil and railroad ties in trenches on and adjacent to the concrete reactor pad were also significantly radiologically impacted. The scope was modified to grout the trenches. Also, additional decontamination activities would not reduce the posting requirements. Therefore, fixed contamination in the reactor pad concrete and the radiologically impacted soil and railroad ties within the grouted trenches were closed in place, and a use restriction was implemented to mitigate potential for future disturbance.

\subsection{Closure Report Contents}

This CR includes the following sections:

- Section 1.0 - Introduction

- Section 2.0 - Closure Activities

- Section 3.0 - Waste Disposition

- Section 4.0 - Closure Verification Results

- Section 5.0 - Conclusions and Recommendations

- Section 6.0 - References 
- Appendix A - Data Quality Objectives

- Appendix B - Sample Analytical Results

- Appendix C - Final Radiological Survey Results

- Appendix D - Waste Disposition Documentation

- Appendix E - Use Restriction Documentation

- Appendix F - Site Closure Photographs

- Appendix G - Record of Technical Change to the SAFER Plan

- Library Distribution List

This report was developed using information and guidance from the following documents:

- SAFER Plan for CAU 115 (NNSA/NSO, 2004)

- Industrial Sites Quality Assurance Project Plan (QAPP) (U.S. Department of Energy, National Nuclear Security Administration Nevada Operations Office [NNSA/NV], 2002)

\subsubsection{Data Quality Objectives}

The data quality objectives used for closure of CAU 115 were presented in Appendix A.1 of the SAFER Plan (NNSA/NSO, 2004) and are included as Appendix A of this report.

The conceptual site model (CSM) was developed and presented in the approved SAFER Plan (NNSA/NSO, 2004). The CSM was based on process knowledge, historical background information, site analysis, and personnel interviews. The CSM assumed that radiologically impacted areas of the facility were limited to the reactor pad, Building 3130 (moveable shed), the shield wall, the concrete pad and pipes on the south side of Building 3113A, the roofs of Buildings 3113 and 3113A, and the floor drain in Room 1. The CSM also assumed that PCBs were present in fluorescent light ballasts and paint, the majority of the solid lead present was not radiologically impacted, $\mathrm{ACM}$ was present as roof mastic sealant and pipe insulation, and mercury was present only in fluorescent light bulbs. The actual site conditions varied from the CSM in that significantly larger areas of radiological contamination were present that were not practical to decontaminate, solid lead items throughout the facility were radiologically contaminated, mercury was present in approximately 800 circuit boards, and cadmium was present in foil surrounding insulated pipes. 
Revision: 0

Date: March 2006

THIS PAGE INTENTIONALLY LEFT BLANK 
This section details the specific activities completed during the closure of CAU 115, deviations from the CAU 115 SAFER Plan as approved, the schedule of completed activities, and the final site plan.

\subsection{DESCRIPTION OF CORRECTIVE ACTION ACTIVITIES}

Closure activities were conducted from September 2004 through September 2005 by the Bechtel Nevada (BN) Environmental Restoration (ER) group. The following sections detail the activities completed during the closure of CAU 115. Photographs in Appendix F of this report document the state of the site before corrective actions were implemented, field work in progress, and the site conditions after completion of work.

\subsubsection{Preplanning and Site Preparation}

Closure activities for CAU 115 were completed using the NDEP-approved SAFER Plan (NNSA/NSO, 2004). Prior to site closure activities, the following documents were prepared:

- National Environmental Policy Act checklist

- Site-Specific Health and Safety Plan

- Field Management Plan

- NNSA/NSO Real Estate/Operations Permits

- BN work control packages

- Sampling and Analysis Plan

- Demolition Plan

- Controlled Explosive Demolition Plan

- Radiological Monitoring Survey Plan

\subsubsection{Hantavirus Cleanup}

Prior to beginning closure activities and throughout closure activities as needed, potential Hantavirus-bearing rodent droppings were treated, removed, and disposed. Hantavirus waste generated from inside a radiologically contaminated area (CA) was managed as low-level waste (LLW). Waste generated outside of a CA was radiologically surveyed. Survey results indicated that the waste was free of radiological impact, and the waste was disposed of as sanitary waste.

\subsubsection{Radiological Surveys}

Extensive radiological surveys were performed prior to and throughout closure activities to determine the presence of contamination, ensure that no contamination was migrating from the CAs, verify the effectiveness of and guide decontamination activities, monitor worker exposure, and determine final waste disposition. All portable survey instruments were calibrated to a known radiological source on a daily basis. Radiological survey reports were completed following procedures outlined in the Nevada/Yucca Mountain Project (NV/YMP) Radiological 
Control Manual (U.S. Department of Energy, Nevada Operations Office [DOE/NV], 2004).

Calibration records, copies of the radiological survey reports, and supporting documentation are on file in the Radiological Control offices in Mercury, Nevada, and are available upon request. A summary of the radiological survey results is included as Appendix $\mathrm{C}$ of this report.

\subsubsection{Characterization of Fluids and Piping}

All piping was inspected for the presence of residual gases, fluids, and pressure before asbestos abatement activities and cutting or removal of pipes. In addition, all electrical lines and utilities were verified to be de-energized.

Prior to removal of equipment, all reservoirs were inspected for the presence of hydraulic oil and lubricating fluids. Fluids were drained and sampled to determine if PCBs were present.

Analytical results for PCBs (Section 3.3, Table 4, Sample CAU1 150503) were below the regulatory limit of 0.74 milligrams per kilogram $(\mathrm{mg} / \mathrm{kg})$, so the fluids were recycled as used oil. Approximately 20 gallons of lubricating fluid and 20 gallons of hydraulic oil were transferred to BN Fleet Services for recycling.

\subsubsection{Removal of Hazardous/Regulated Waste}

Hazardous and regulated materials were removed from Buildings 3113, 3113A, and 3113B and related facilities prior to demolition of the structures.

\subsubsection{Asbestos-Containing Material}

The roof mastic sealant located around roof penetrations on Buildings 3113 and 3113A was determined to be ACM. The material was removed and double bagged. Radiological surveys established that the roof mastic sealant was radiologically impacted and was therefore managed as asbestiform low-level waste (ALLW). The material is currently staged onsite and will be transported to the Area 5 Radioactive Waste Management Complex (RWMC) for disposal by the end of fiscal year (FY) 2006. Waste disposition documentation for the ACM will be provided to the NDEP following disposal.

A total of approximately 520 linear feet (ft) of piping insulated with friable asbestos was removed and double wrapped in plastic. Other ACM, including a fire hose, two fire suits, electrical cables, pipe elbows, and putty, were removed and double bagged. The ACM was analyzed by In-Situ Object Counting System (ISOCS) to determine whether it is radiologically impacted. After the results are evaluated, any ACM that is free of radiological impact will be transported to the Area 23 Sanitary Landfill for disposal. Any ACM that is determined to be radiologically impacted will be disposed of as ALLW at the Area 5 RWMC. A total of approximately 75 cubic feet $\left(\mathrm{ft}^{3}\right)$ of ACM is currently staged onsite awaiting disposal. The waste will be disposed of appropriately based on ISOCS results by the end of FY 2006. Waste disposition documentation for the ACM will be provided to the NDEP following disposal.

In addition, ACM piping covered with insulating foil was removed and double bagged. The foil was sampled and determined to be hazardous (Section 2.1.5.4), and radiological surveys were performed. Depending on the results of the radiological surveys, the material was classified as either hazardous waste (HW) or asbestiform mixed waste (MW). A total of approximately $500 \mathrm{ft}^{3}$ of the material was classified as HW and transported to the Area $5 \mathrm{HW}$ Storage Unit. It was shipped to a permitted offsite facility for treatment and disposal on April 4, 2005. A total of 
approximately $1,330 \mathrm{ft}^{3}$ of the material was classified as asbestiform MW and transported to the Area 5 TRU Pad. It was shipped to a permitted offsite facility for treatment and disposal on January 24, 2006.

\subsubsection{Lead}

Solid lead in the form of sheets, doors, bricks, and wool was used as shielding material in Building 3113. Other lead items included a lead collar and a lead-lined box. All lead-containing materials were removed and surveyed for radiological contamination. The lead was determined to be radiologically impacted and was managed as MW. A total of approximately 76,300 pounds of lead that was radiologically contaminated was transported to the Area 5 TRU Pad. The lead was shipped to a permitted offsite facility for treatment and disposal on September 21, 2005.

Field screening using x-ray fluorescence (XRF) indicated that the paint in Building 3113 contained lead. To determine the waste disposal pathway for the paint, paint samples were collected and analyzed by Toxicity Characterization Leaching Procedure (TCLP) for lead. Leachable lead concentrations in the paint did not exceed the RCRA regulatory level of 5.0 milligrams per liter $(\mathrm{mg} / \mathrm{L})$ for hazardous waste (Section 3.3, Table 4, Samples 1153113-1, 1153113B-1, 1153113B-2, and 115N-1).

Swipe samples indicated several surfaces in the facility were contaminated with lead dust. Contaminated surfaces were washed and/or vacuumed with a high-efficiency particulate air (HEPA) vacuum to remove surface contaminants and loose paint chips prior to demolition activities.

\subsubsection{Mercury}

Approximately 800 circuit boards containing mercury were removed and containerized in drums. The circuit boards were surveyed and determined to be free of radiological contamination. The drums were transported to the Area $5 \mathrm{HW}$ Storage Unit. The circuit boards were shipped to a permitted offsite facility for treatment and disposal on September 13, 2005.

\subsubsection{Cadmium}

Insulating foil covering piping and loose foil scattered throughout the facility was identified as possibly containing hazardous constituents. Two representative samples of the foil were collected and analyzed for TCLP metals. The sample results indicated that the foil contained cadmium above the RCRA regulatory level of $1.0 \mathrm{mg} / \mathrm{L}$ for hazardous waste (Section 3.3, Table 4, Samples CAU115050101 and CAU115050201). The foil was removed with the piping/conduit and asbestos that it was attached to, containerized in drums, and radiologically surveyed. A total of approximately $1,330 \mathrm{ft}^{3}$ of the material was radiologically impacted and was transported to the Area 5 TRU Pad. The material was shipped as MW to a permitted offsite facility for treatment and disposal on January 24, 2006. A total of approximately $500 \mathrm{ft}^{3}$ of the material was free of radiological contamination and was transported to the Area $5 \mathrm{HW}$ Storage Unit. The material was shipped as HW to a permitted offsite facility for treatment and disposal on September 13, 2005. 


\subsubsection{PCBs}

Equipment containing hydraulic oil was suspected to contain PCBs in concentrations greater than the land disposal limits. All fluids were drained, and one representative sample was collected and analyzed for PCBs. PCBs were not detected at concentrations exceeding the regulatory limit of $0.74 \mathrm{mg} / \mathrm{kg}$ (Section 3.3, Table 4, Sample CAU1150503), and the fluids were transferred to BN Fleet Services for recycling.

Previous sample results indicated that paint on many of the building surfaces contained PCBs at concentrations exceeding the NTS sanitary landfill limit. A total of approximately $15 \mathrm{ft}^{3}$ of paint was removed from the building surfaces and reactor pad by scabbling and HEPA vacuums and was containerized in drums. Radiological surveys indicated that the paint was also radiologically impacted. The paint chips were managed as radioactive PCB bulk-product waste and are currently staged onsite. The paint chips will be transported to the NTS RWMC for disposal by the end of FY 2006. Waste disposition documentation will be provided to the NDEP following disposal.

In addition, 12 fluorescent light ballasts containing PCBs were removed and managed as Toxic Substances Control Act (TSCA) waste. The ballasts were found to be leaking, transported in drums to a permitted offsite facility on January 3, 2006, and incinerated on January 22, 2006.

\subsubsection{Universal Waste}

Six lead acid batteries and 38 fluorescent light bulbs were removed from the facility and managed as universal waste (UW). The batteries and fluorescent light bulbs were transported to an offsite facility for recycling. Fire extinguishers throughout the facility were also removed and transported to the Mercury fire station for recharging and reuse.

\subsubsection{Removal of Radiologically Impacted Soil}

A total of approximately $100 \mathrm{ft}^{3}$ of radiologically impacted soil was removed from an area adjacent to the northwest corner of the concrete pad and packaged in B25 boxes. This soil was outside the CAU boundary but was removed as a BMP due to elevated radiological levels. The soil is currently staged onsite and will be disposed of as LLW at the NTS RWMC by the end of FY 2006. Waste disposition documentation for the soil will be provided to the NDEP following disposal.

A total of approximately $125 \mathrm{ft}^{3}$ of radiologically impacted soil and tumbleweeds was removed from the trenches located on the reactor pad and packaged in B25 boxes. The soil is currently staged onsite and will be disposed of as LLW at the NTS RWMC by the end of FY 2006. Waste disposition documentation for the soil will be provided to the NDEP following disposal. The trenches were grouted with concrete to increase shielding of the remaining contamination and allow the area to be posted as an Underground Radioactive Materials Area (URMA).

\subsubsection{Characterization and Decontamination of Radiologically Impacted Surfaces}

Concrete core samples were collected from the shield wall to determine compressive strength to refine the demolition strategy. Concrete core samples from the shield wall, the roof of Building 3113, the reactor pad, and other concrete surfaces were analyzed by ISOCS to determine the levels of radiological contamination and guide decontamination and demolition activities. 
Surfaces impacted by radionuclides, including the roof of Building 3113 and the reactor pad, were decontaminated using a combination of aggressive (e.g., scabbling) and non-aggressive (e.g., HEPA vacuuming) methods. Fixed contamination within the roof paint was removed using aggressive methods while decontamination activities on the reactor pad were limited to removal of loose contamination. ISOCS data indicated that the pad was activated to a depth of $2 \mathrm{ft}$. Therefore, aggressive decontamination to remove the pad surface would not have lowered dose readings to below regulatory levels.

\subsubsection{Demolition of Aboveground Structures}

Buildings $3113,3113 \mathrm{~A}$, and 3113B were torn down using conventional demolition methods. As a BMP, Building 3130 (moveable shed), Building 3134 (hydrogen fill stations), the dewar, the exhaust stack system, and the Perkin unit were also removed or demolished by conventional means. Heavy equipment, including demolition shears, were used to knock down and size-reduce the buildings and associated structures. Radiological surveys were performed on the demolition debris.

Due to the concrete thickness and heavy reinforcements, the shield wall was demolished using explosives. A BN subcontractor was responsible for the demolition activities along with the size-reduction and staging of the debris. After demolition, the debris from the shield wall was radiologically surveyed.

The dewar, which had been used to store liquid nitrogen for the nuclear reactor tests, was removed as a BMP. The top of the dewar was accessed, and the Perlite insulation located in the space between the inner and outer walls of the dewar was sampled and analyzed by ISOCS to determine appropriate handling and disposal. The Perlite was not radiologically impacted above the Area 9 U10c disposal limits. Demolition shears were used to remove the top of the dewar, and a total of approximately $7,500 \mathrm{ft}^{3}$ of Perlite insulation was removed and transported as sanitary waste at the Area 9 U10c Landfill from July 11, 2005, through September 1, 2005. The remaining metal structure was torn down and size-reduced using shears and torches, and the debris was screened for radiological contamination.

During demolition activities, radiological surveys were performed on the demolition debris from Buildings 3113, 3113A, 3113B, 3130, 3134, the dewar, the exhaust stack system, the Perkin unit, and the shield wall. The debris was segregated into sanitary waste and LLW streams. A total of approximately $13,500 \mathrm{ft}^{3}$ of demolition debris was disposed of at the Area 9 U10c Landfill from February 14, 2005, through June 7, 2005. A total of approximately $40,000 \mathrm{ft}^{3}$ of radiologically impacted debris is currently staged onsite. The radiologically impacted debris will be disposed of as radioactive PCB bulk-product waste due to the fact that the majority of the facility was painted with paint containing PCBs. The demolition debris will be disposed of at the NTS RWMC by the end of FY 2006. Waste disposition documentation for the debris will be provided to the NDEP following disposal.

After demolition of the aboveground structures, remaining piping was cut at the ground surface. The pipe ends, floor drains, and other openings were sealed with cement. 


\subsubsection{Final Radiological Survey}

After all demolition activities were completed and waste and debris was removed or staged, final radiological surveys were performed to document final site conditions and establish appropriate radiological controls. A summary of the results is included as Appendix C of this report.

\subsubsection{Implementation of Use Restriction}

Due to radiological activation of the concrete reactor pad and remaining radiologically impacted soil and railroad ties, clean closure was not practical for CAU 115. A use restriction was established for the remaining radiological contamination in the reactor pad. The locations of the corners of the use-restricted area are listed in Table 1. Figure 3 shows the boundary of the userestricted area.

The reactor pad was posted as an URMA. Radiological postings were installed every $100 \mathrm{ft}$. In addition, use restriction warning signs were posted on T-posts near each of the four corners of the reactor pad according to the FFACO Use Restriction Posting Guidance (FFACO, 2003).

TABLe 1. CAU 115 USE-Restricted AREA, UTM, Zone 11, NAD 27, Meters

\begin{tabular}{|c|c|c|}
\hline CoRNER & NoRTHING & EASTING \\
\hline Northeast & $4,076,007.5$ & $566,256.7$ \\
\hline Southeast & $4,075,962.9$ & $566,256.7$ \\
\hline Southwest & $4,075,962.9$ & $566,230.3$ \\
\hline Northwest & $4,076,007.5$ & $566,230.3$ \\
\hline
\end{tabular}

\subsection{DEVIATIONS FROM THE SAFER Plan AS APPROVED}

Closure activities followed the approach specified in the SAFER Plan (NNSA/NSO, 2004). Exceptions to the original SAFER Plan were documented in a ROTC, which is included as Appendix $\mathrm{G}$ of this report. Radiological surveys and ISOCS core samples collected during closure activities revealed conditions that were not anticipated during project planning, resulting in a ROTC to the SAFER Plan. The ROTC addressed the following:

- Clean closure would not be achieved for CAU 115 due to radiological activation of the concrete reactor pad as determined by ISOCS analysis. The reactor pad would instead be posted as an URMA, and a use restriction would be implemented. A final radiological survey would be conducted to establish proper controls.

- The railroad trenches would be filled with grout, rather than removing the fixed contamination present on the interior surface, since removing fixed contamination would not remove the posting requirements on the reactor pad due to radiological activation of the concrete. Secondary reasons for filling the trenches included covering and shielding the fixed contamination located in the trenches, reducing worker exposure, and shortening the duration of decontamination activities.

- The dewar would be removed as a BMP. 
Date: March 2006

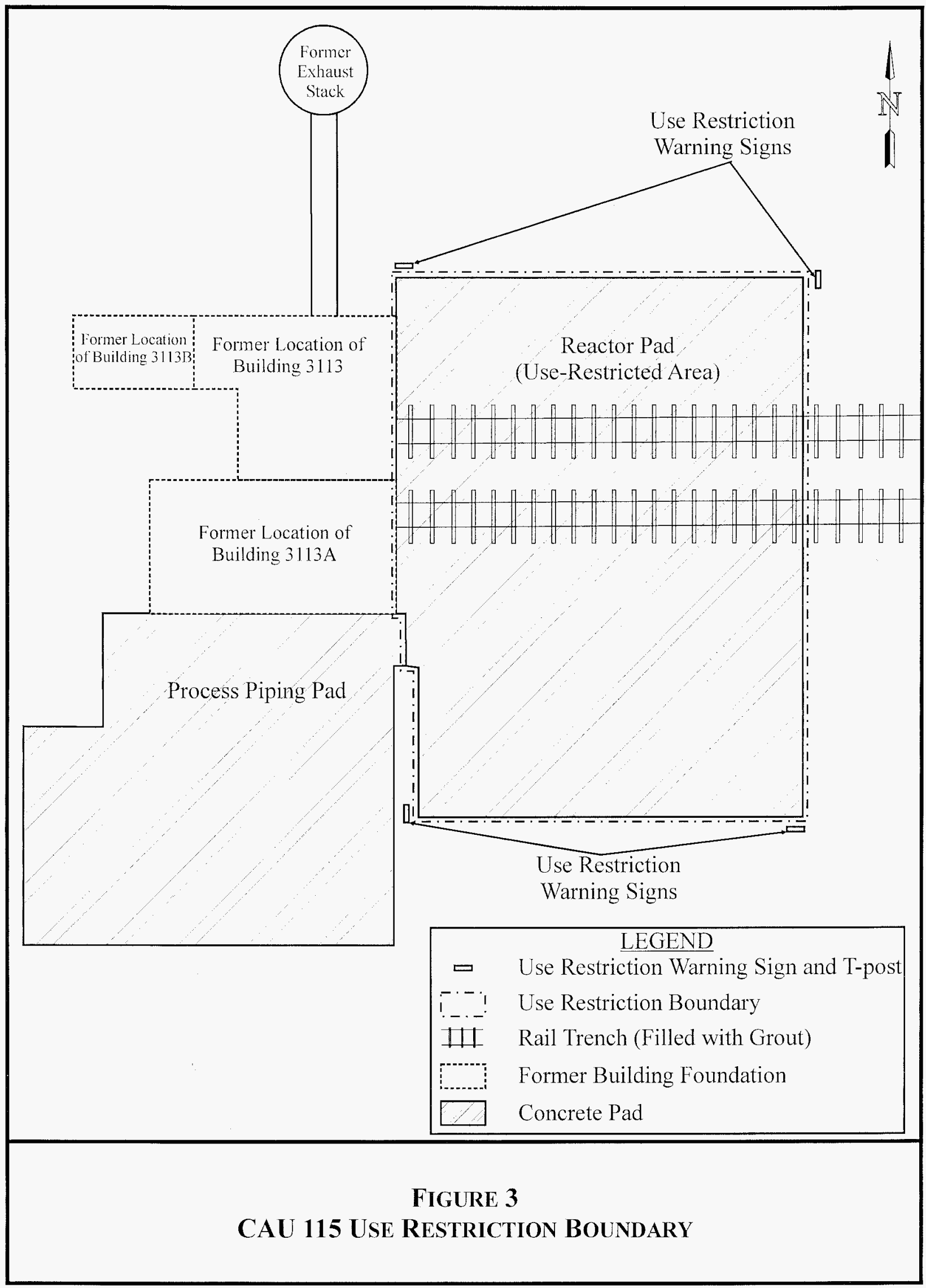




\subsection{CoRRective ACTION SCHEdUle AS COMPLETED}

The closure activities began in September 2004 and were completed in September 2005. Details of the closure field activities schedule are provided in Table 2.

Table 2. CAU 115 Closure Activities Schedule

\begin{tabular}{|l|l|l|}
\hline \multicolumn{1}{|c|}{ ACTIVITY } & \multicolumn{1}{|c|}{ START DATE } & \multicolumn{1}{c|}{ END DATE } \\
\hline Mobilization and Site Setup & September 30, 2004 & January 12, 2005 \\
\hline Removal of Hazardous and Regulated Waste & January 12, 2005 & March 24, 2005 \\
\hline $\begin{array}{l}\text { Decontamination Activities and Removal of Radiologically } \\
\text { Impacted Soil }\end{array}$ & March 29, 2005 & June 03, 2005 \\
\hline Demolition of Aboveground Structures & May 12, 2005 & September 07, 2005 \\
\hline Final Radiological Survey & July 07, 2005 & July 18, 2005 \\
\hline Demobilization & September 07, 2005 & September 13, 2005 \\
\hline
\end{tabular}

\subsection{Site Plan/Survey Plat}

Remaining radiological contamination for CAU 115 was closed in place with administrative controls (i.e., a use restrictions was implemented). A figure showing the locations of the surveyed points delineating the use-restricted area is included in Appendix $E$ of this report. 


\subsection{WASTE DISPOSITION}

This section describes the waste streams generated during closure activities and their final disposition. Waste streams generated included ACM, ALLW, asbestiform MW, HW, LLW, MW, TSCA regulated waste, used oil, UW, and non-hazardous sanitary waste. All waste was characterized and managed according to state and federal regulations, U.S. Department of Energy (DOE) orders, and BN procedures. Waste characterization sample analytical results are included in Appendix B of this report. Waste disposition is summarized in Table 3 and discussed in detail in the following sections. Waste disposition documentation is included in Appendix D of this report.

Table 3. CAU 115 Waste Disposition Summary

\begin{tabular}{|c|c|c|c|}
\hline DESCRIPTION & AMOUNT & Waste TyPE & DISPOSITION \\
\hline Non-Impacted ACM & Amount Pending* & $\mathrm{ACM}$ & $\begin{array}{l}\text { Staged onsite pending ISOCS results } \\
\text { for disposal at the Area } 23 \text { Sanitary } \\
\text { Landfill by the end of FY } 2006\end{array}$ \\
\hline Radiologically Impacted ACM & Amount Pending* & ALLW & $\begin{array}{l}\text { Staged onsite pending ISOCS results } \\
\text { for disposal at the Area } 5 \text { RWMC by } \\
\text { the end of FY } 2006\end{array}$ \\
\hline $\begin{array}{l}\text { Radiologically Impacted ACM } \\
\text { with Cadmium Foil }\end{array}$ & $1,330 \mathrm{ft}^{3}$ & $\begin{array}{l}\text { Asbestiform } \\
\text { MW }\end{array}$ & $\begin{array}{l}\text { Transported to a permitted offsite } \\
\text { facility for treatment and disposal on } \\
01 / 24 / 2006\end{array}$ \\
\hline Cadmium Foil & $500 \mathrm{ft}^{3}$ & \multirow{3}{*}{ HW } & \multirow{3}{*}{$\begin{array}{l}\text { Transported to a permitted offsite } \\
\text { facility for treatment and disposal on } \\
09 / 13 / 2005\end{array}$} \\
\hline Circuit Boards & 800 circuit boards & & \\
\hline Vacuum Tubes & 4 tubes & & \\
\hline $\begin{array}{l}\text { Soil from northwest corner of } \\
\text { reactor pad }\end{array}$ & $100 \mathrm{ft}^{3}$ & \multirow{3}{*}{ LLW } & \multirow{3}{*}{$\begin{array}{l}\text { Staged onsite for disposal at the NTS } \\
\text { RWMC by the end of FY } 2006\end{array}$} \\
\hline $\begin{array}{l}\text { Soil and tumbleweeds from } \\
\text { railroad trenches }\end{array}$ & $125 \mathrm{ft}^{3}$ & & \\
\hline Construction Debris & $40,000 \mathrm{ft}^{3}$ & & \\
\hline Solid Lead & 76,300 pounds & MW & $\begin{array}{l}\text { Transported to a permitted offsite } \\
\text { facility for treatment and disposal on } \\
09 / 21 / 2005\end{array}$ \\
\hline Fluorescent Light Ballasts & 12 bulbs & TSCA Waste & $\begin{array}{l}\text { Transported to a permitted offsite } \\
\text { facility on } 01 / 03 / 2006 \text { for } \\
\text { incineration on } 01 / 22 / 2006\end{array}$ \\
\hline Paint Chips & $15 \mathrm{ft}^{3}$ & $\begin{array}{l}\text { Radioactive } \\
\text { PCB Bulk- } \\
\text { Product Waste }\end{array}$ & $\begin{array}{l}\text { Staged onsite for disposal at the NTS } \\
\text { RWMC by the end of FY } 2006\end{array}$ \\
\hline Lubricating Fluid & 20 gallons & \multirow{2}{*}{ Used Oil } & \multirow{2}{*}{ Recycled at BN Fleet Services } \\
\hline Hydraulic Oil & 20 gallons & & \\
\hline Lead Acid Batteries & 6 batteries & \multirow{2}{*}{ UW } & \multirow{2}{*}{ Recycled at an offsite facility } \\
\hline Fluorescent Light Bulbs & 38 bulbs & & \\
\hline $\begin{array}{l}\text { Construction Debris and } \\
\text { Perlite }\end{array}$ & $21,000 \mathrm{ft}^{3}$ & Sanitary Waste & $\begin{array}{l}\text { Disposed of at the Area } 9 \mathrm{U} 10 \mathrm{c} \\
\text { Landfill }\end{array}$ \\
\hline
\end{tabular}

* A total of $75 \mathrm{ft}^{3}$ of ACM is currently staged onsite. After review of ISOCS analysis results, the waste will either be disposed of as ACM at the Area 23 Sanitary Landfill or as ALLW at the Area 5 RWMC by the end of FY 2006. 


\subsection{Waste Minimization}

Industry standard waste minimization practices were applied throughout the course of closure activities. These practices included:

- Using highly efficient industry standard decontamination tools that removed radiologically impacted material

- Using field instrumentation (i.e., survey instruments, ISOCS analysis) to identify the areas requiring decontamination

- Using a portable XRF unit to field screen for lead contamination

- Using laboratory analysis and ISOCS to correctly characterize and classify waste streams

- Recycling items that had potential for future use, including lubricating fluid, hydraulic oil, lead acid batteries, and fluorescent light bulbs

\subsection{WASte MANAGEMENT}

All waste was managed according to applicable state and federal regulations, DOE orders, the CAU 115 SAFER Plan (NNSA/NSO, 2004), BN procedures, and company directives.

Waste management areas (WMAs) were established throughout the project, as needed. All WMAs were identified with appropriate signs and boundaries to restrict unauthorized access. The WMAs were inspected on a weekly or monthly basis, as required, to ensure that all containers were intact, not leaking, and not exceeding storage duration times as specified by state regulations and BN operating procedures. Applicable WMAs were posted as Radioactive Materials Areas (RMAs) whenever radiological waste was stored in the area. Upon removal of radiologically impacted waste, the RMA was surveyed and de-posted.

Waste containers were purchased either new or reconditioned. All containers were inspected prior to use to verify that they were in good condition (e.g., no leaks, rust, or dents), lined or made of material that would not react with the waste, and met U.S. Department of Transportation requirements. The containers remained closed while stored unless waste was being added or removed. Containers were also handled in such a manner that the integrity of the container was not compromised. Drums containing liquid regulated waste were stored on spill containment pallets. Appropriate labels were affixed and relevant information was marked on the containers with an indelible marker. All information was legible and clearly visible.

\subsection{Waste Characterization}

Waste streams were characterized according to the CAU 115 SAFER Plan (NNSA/NSO, 2004), BN Solid Waste Operations, BN Hazardous Waste Operations, and BN Waste Generator Services procedures. Laboratory samples were collected, sealed with a custody seal, cooled to four degrees Celsius, and logged on a chain of custody. The waste characterization samples collected during closure activities are listed in Table 4, and summary results are included in Appendix B of this report. Waste was also screened for radiological contamination using radiological survey instruments and ISOCS analysis. 


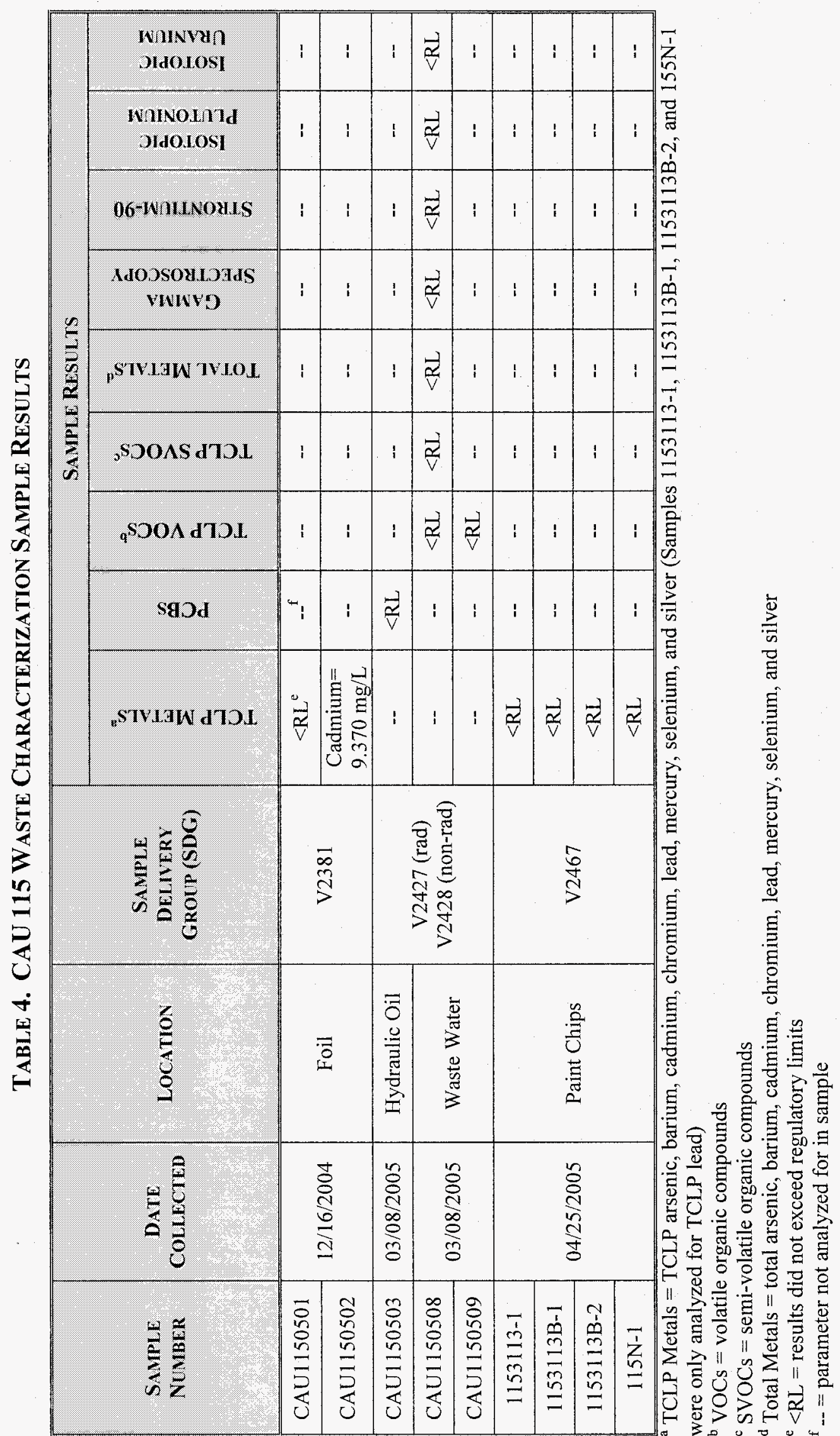




\subsection{WASTe Streams AND DisPosaL}

Waste streams generated during closure activities at CAU 115 included ACM, ALLW, asbestiform MW, HW, LLW, MW, TSCA regulated waste, used oil, UW, and non-hazardous sanitary waste. Waste disposition documentation is included in Appendix D of this report.

\subsubsection{Asbestos-Containing Material}

A total of approximately $75 \mathrm{ft}^{3}$ of ACM, including pipe insulation, a fire hose, two fire suits, electrical cables, pipe elbows, and putty, were analyzed by ISOCS to determine whether they are radiologically impacted. The material is currently being stored onsite in drums. After the ISOCS results are evaluated, ACM that is not radiologically impacted will be transported to the Area 23 Sanitary Landfill for disposal. The ACM will be disposed of by the end of FY 2006, and waste disposition documentation will be provided to the NDEP following disposal.

\subsubsection{Asbestiform Low-Level Waste}

Any of the ACM that is currently onsite awaiting ISOCS results evaluation (Section 3.4.1) that is determined to be radiologically impacted above sanitary landfill limits will be disposed of as ALLW at the Area 5 RWMC by the end of FY 2006, and waste disposition documentation will be provided to the NDEP following disposal.

\subsubsection{Asbestiform MW}

A total of approximately $1,330 \mathrm{ft}^{3}$ of ACM piping covered with foil containing hazardous levels of cadmium was radiologically impacted. The material was accumulated in drums and transported to the Area 5 TRU Pad. The MW was shipped to a permitted offsite facility for treatment and disposal on January 24, 2006.

\subsubsection{Hazardous Waste}

HW that was determined to be free of radiological impact included approximately $500 \mathrm{ft}^{3}$ of cadmium foil, approximately 800 mercury-containing circuit boards, and 4 vacuum tubes. HW was stored in drums and transported to the Area $5 \mathrm{HW}$ Storage Unit. It was shipped to a permitted offsite facility for treatment and disposal on September 13, 2005.

\subsubsection{Low-Level Waste}

A total of approximately $225 \mathrm{ft}^{3}$ of LLW was accumulated in B25 boxes and is currently staged onsite to be transported to the NTS RWMC for disposal by the end of FY 2006. This included approximately $100 \mathrm{ft}^{3}$ of soil excavated from a location near the northwest corner of the reactor pad and approximately $125 \mathrm{ft}^{3}$ of soil and tumbleweeds removed from the railroad trenches on the reactor pad. Waste disposition documentation will be provided to the NDEP following disposal.

\subsubsection{Mixed Waste}

A total of approximately 76,300 pounds of radiologically impacted solid lead, including bricks, wool, doors, and a lead-lined box, was packaged in drums and transported to the Area 5 TRU 
Pad. The lead was shipped to a permitted offsite facility for treatment and disposal on September 21, 2005.

\subsubsection{TSCA-Regulated Waste}

A total of approximately $15 \mathrm{ft}^{3}$ of paint chips impacted with PCBs and radionuclides was managed as radioactive PCB bulk-product waste. The paint was packaged in drums and is currently staged onsite. It will be transported to the NTS RWMC for disposal by the end of FY 2006. In addition, 12 fluorescent light ballasts containing PCBs were transported in drums to a permitted offsite facility on January 3,2006, and incinerated on January 22, 2006.

In addition, a total of approximately $40,000 \mathrm{ft}^{3}$ of radiologically impacted construction debris from the demolition of structures at Test Cell $\mathrm{A}$ is currently staged onsite. The majority of the facility was painted with paint containing PCBs, so the debris will be disposed of as radioactive PCB bulk-product waste. The demolition debris will be disposed of at the NTS RWMC by the end of FY 2006. Waste disposition documentation for the debris will be provided to the NDEP following disposal.

\subsubsection{Used Oil}

Fluids that were drained from equipment reservoirs were determined to be free of radiological and hazardous constituents and were recycled as used oil. Approximately 20 gallons of lubricating fluid and 20 gallons of hydraulic oil were transferred to BN Fleet Services for recycling.

\subsubsection{Universal Waste}

Various forms of UW were generated during the closure of CAU 115. Six lead acid batteries and 38 fluorescent light bulbs were removed, surveyed, and managed as UW. The batteries and fluorescent light bulbs were transported to an offsite facility for recycling. Fire extinguishers throughout the facility were also removed and transported to the Mercury fire station for recharging and reuse.

\subsubsection{Sanitary Waste}

A total of approximately $21,000 \mathrm{ft}^{3}$ of sanitary construction debris and Perlite was generated during demolition activities at CAU 115 . The debris was determined to be sanitary waste, based on radiological surveys. All sanitary waste was transported in end-dump trucks to the Area 9 U10c Landfill for disposal. 


\section{THIS PAGE INTENTIONALLY LEFT BLANK}




\subsection{CLOSURE VERIFICATION RESULTS}

To document final site conditions and establish appropriate radiological controls, final radiological surveys were performed. A summary of the results is included in Appendix C of this report.

\subsection{Data Quality Assessment}

Accurate and defensible analytical data were collected to verify that waste was properly characterized, managed, and disposed of during CAU 115 closure activities. The following sections describe the quality assurance $(\mathrm{QA})$ / quality control $(\mathrm{QC})$ procedures, data validation process, and a reconciliation of the CSM with actual findings during CAU 115 closure activities. More detail on the QA/QC procedures for CAU 115 can be found in the CAU 115 SAFER Plan (NNSA/NSO, 2004).

\subsubsection{Quality Assurance/Quality Control Procedures}

Waste characterization samples were collected with disposable polyethylene scoops and placed in appropriately labeled sample containers secured with custody seals. All samples were labeled with a unique sample number, placed on ice, and transported under a chain of custody. Standard QA/QC samples were collected (i.e., one blind duplicate per 20 samples). Samples were analyzed by BN contract laboratories. Analytical results were validated at the laboratory using stringent QA/QC procedures, including matrix spike/matrix spike duplicates, spiked surrogate recovery analysis, verification of analytical results, and data quality indicator requirements. Detailed information regarding the QA/QC program can be found in the Industrial Sites QAPP (NNSA/NV, 2002).

\subsubsection{Data Validation}

Data validation was performed according to the Industrial Sites QAPP (NNSA/NV, 2002) which is based on the U.S. Environmental Protection Agency (EPA) functional guidelines for data quality (EPA, 1994 and 1999). Data were reviewed to ensure that samples were appropriately processed and analyzed, and that the results are valid. All sample data were internally validated by qualified BN personnel at the Tier I and Tier II levels. No anomalies were discovered in the data that would discredit any of the waste characterization or verification sample results. While only summary laboratory QC data for waste characterization samples are included in Appendix B of this report, the complete data set, including validation reports for waste characterization samples, is maintained in the BN ER project files and available upon request.

\subsubsection{Conceptual Site Model}

The CSM was developed and presented in the approved SAFER Plan (NNSA/NSO, 2004). The CSM was based on process knowledge, historical background information, site analysis, and personnel interviews. The CSM assumed that radiologically impacted areas of the facility were limited to the reactor pad, Building 3130 (moveable shed), the shield wall, the concrete pad and pipes on the south side of Building 3113A, the roofs of Buildings 3113 and 3113A, and the floor drain in Room 1. The CSM also assumed that PCBs were present in fluorescent light ballasts and paint, the majority of the solid lead present was not radiologically impacted, ACM was 
present as roof mastic sealant and pipe insulation, and mercury was present only in fluorescent light bulbs. The actual site conditions varied from the CSM in that significantly larger areas of radiological contamination were present that were not practical to decontaminate, solid lead items throughout the facility were radiologically contaminated, mercury was present in approximately 800 circuit boards, and cadmium was present in foil surrounding insulated pipes.

\subsection{USE RESTRICTION}

A use restriction has been implemented for CAU 115. Fixed radioactive contamination in the concrete reactor pad and railroad trenches that was not practical to remediate was left in place. Radiological postings identify the reactor pad as an URMA, and use restriction warning signs were posted on T-posts at each of the four corners of the reactor pad to warn against intrusive activity according to the FFACO Use Restriction Posting Guidance (FFACO, 2003). The CAU Use Restriction Information form and a figure showing the locations of the surveyed points delineating the use-restricted area are included in Appendix E of this report. Annual site inspections will be performed to ensure that all signs are in good repair and that the use restriction has been maintained. Details on the post-closure requirements are included in Section 5.2 . 


\subsection{CONCLUSIONS AND RECOMMENDATIONS}

\subsection{Conclusions}

The following site closure activities were performed at CAU 115 and are documented in this CR:

- All hazardous and regulated waste was removed from the facility and disposed, including the following:

- ACM (pipe insulation, roof mastic sealant)

- Solid lead (bricks, wool)

- Mercury (circuit boards)

- Cadmium (foil covering insulated pipes)

- PCBs (fluorescent light ballasts, paint)

- Buildings 3113, 3113A, and 3113B and related equipment and structures were partially decontaminated, demolished, and disposed.

- The reactor concrete pad was characterized and partially decontaminated.

- The dewar was removed as a BMP.

- Final radiological surveys were performed to establish appropriate radiological controls and document final site conditions.

- Radiological postings and use restriction warning signs were installed.

\subsection{PoST-CLOSURE REQUIREMENTS}

\subsubsection{Inspections}

Inspections will be performed annually as part of the Decontamination and Decommissioning Program under the Surveillance and Maintenance Plan. Inspections will consist of visual observations to verify that all signs are in good repair and that the use restriction has been maintained. If any maintenance or repair requirements are identified during the inspection, funding will be requested and the repairs scheduled. Any repair or maintenance performed at this site shall be documented in writing at the time of the repair and included in a letter report.

\subsection{RECOMMENDATIONS}

Since closure activities for CAU 115 have been completed following the NDEP-approved SAFER Plan (NNSA/NSO, 2004) as documented in this report, NNSA/NSO requests the following:

1. A Notice of Completion be provided by the NDEP to the NNSA/NSO for the closure of CAU 115.

2. The transfer of CAU 115 from Appendix III to Appendix IV, Closed Corrective Action Units, of the FFACO (FFACO, 1996). 
Closure Report - CAU 115

Section: Conclusions \& Recom

Revision: 0

Date: March 2006

THIS PAGE INTENTIONALLY LEFT BLANK 


\subsection{REFERENCES}

DOE/NV, see U.S. Department of Energy, Nevada Operations Office.

EPA, see U.S. Environmental Protection Agency.

Federal Facility Agreement and Consent Order. 1996 (as amended). Agreed to by the state of Nevada, the U.S. Department of Energy, and the U.S. Department of Defense.

Federal Facility Agreement and Consent Order. 2003. Use Restriction Posting Guidance.

FFACO, see Federal Facility Agreement and Consent Order.

NNSA/NSO, see U.S. Department of Energy, National Nuclear Security Administration Nevada Site Office

NNSA/NV, see U.S. Department of Energy, National Nuclear Security Administration Nevada Operations Office

U.S. Department of Energy, National Nuclear Security Administration Nevada Operations Office. 2002. Nevada Environmental Restoration Project Industrial Sites Quality Assurance Project Plan, Nevada Test Site, Nevada. DOE/NV--372--REV 3. Las Vegas, NV.

U.S. Department of Energy, National Nuclear Security Administration Nevada Site Office. 2004. Streamlined Approach for Environmental Restoration Plan for Corrective Action Unit 115: Area 25 Test Cell A Facility, Nevada Test Site, Nevada. DOE/NV--987-REV 1. Las Vegas, NV.

U.S. Department of Energy, Nevada Operations Office. 2004. NV/YMP Radiological Control Manual. DOE/NV/11718--079-REV 5. Las Vegas, Nevada.

U.S. Environmental Protection Agency. 1994. Guidance for the Data Quality Objectives Process, EPA QA/G-4. Washington D.C.

U.S. Environmental Protection Agency. 1999. Contract Laboratory Program National Functional Guidelines for Organic Data Review, EPA540/R-99/008. Washington D.C. 
Closure Report - CAU 115

Section: References

Revision: 0

Date: March 2006

THIS PAGE INTENTIONALLY LEFT BLANK 


\section{APPENDIX A*}

\section{DATA QUALITY OBJECTIVES}

* As presented and published in the approved Streamlined Approach for Environmental Restoration Plan for Corrective Action Unit 115: Area 25 Test Cell A Facility, Nevada Test Site, Nevada, 2004, DOE/NV--987-REV 1. Las Vegas, NV. 
Closure Report - CAU 115

Section: Appendix A

Revision: 0

Date: March 2006

THIS PAGE INTENTIONALLY LEFT BLANK 
SAFER Plan - CAU 115

Section: Appendix A.1

Revision: 1

Date: December 2004

APPENDIX A.1 DATA QUALITY OBJECTIVES

A.1-1 
SAFER Plan - CAU 115

Section: Appendix A.1

Revision: 1

Date: December 2004

THIS PAGE INTENTIONALLY LEFT BLANK

A.1-2 
SAFER PLAN - CAU 115

Section: Appendix A.1

Revision:

Date: December 2004

APPENDIX A.1

DATA QUALITY OBJECTIVES FOR CORRECTIVE ACTION UNIT 115: AREA 25 TEST CELL A FACILITY

A. 1-3 


\section{TABLE OF CONTENTS}

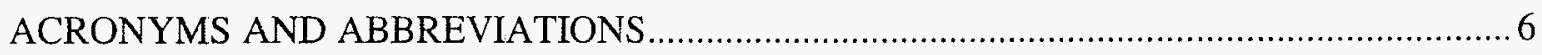

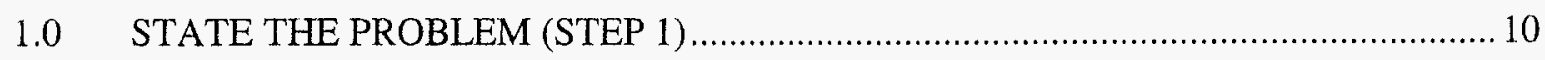

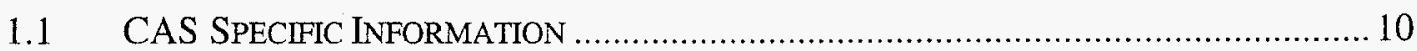

1.1.1 CAS 25-41-04, Test Cell A Facility .................................................. 10

1.1.2 CAS 25-99-04, Asbestos Wrapped Piping (Exterior) ............................ 11

1.1.3 CAS 25-99-05, Asbestos Wrapped Piping (Interior) .............................. 11

1.1.4 CAS 25-99-06, Asbestos Wrapped Piping (Roof) ................................. 11

1.2 DEVELOP/REFINE THE CONCEPTUAL MODEL .............................................. 12

1.2.1 Test Cell A Deactivation and Decommissioning Conceptual Site Model 12

1.3 SECONDARy CONCEPTUAL SITE MOdELS FOR CORRECTIVE ACTION UNIT $115 \ldots 14$

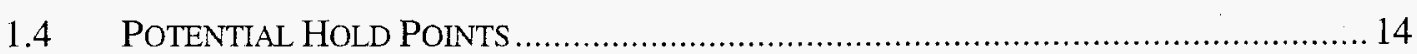

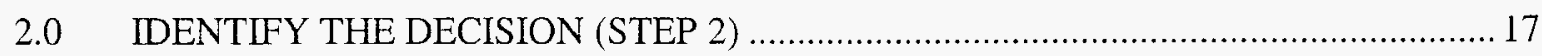

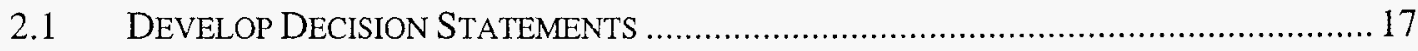

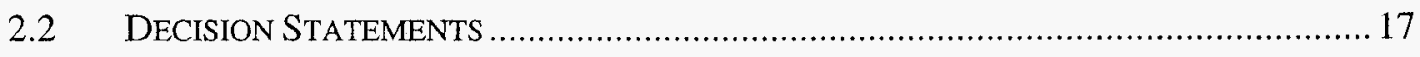

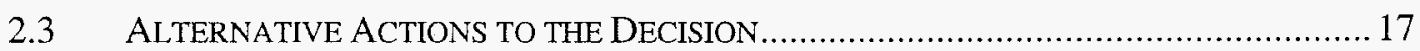

3.0 IDENTIFY THE INPUTS TO THE DECISION (STEP 3) ..................................... 19

3.1 IDENTIFY THE INFORMATION INPUTS NEEDED AND RESOLVE THE DECISION .......... 19

3.1.1 Quantitative Data......................................................................... 20

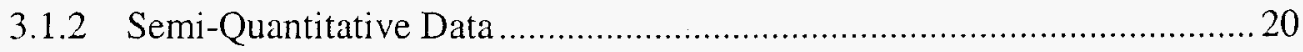

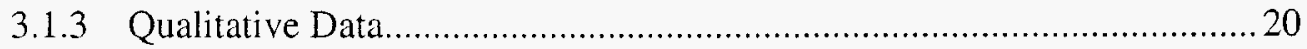

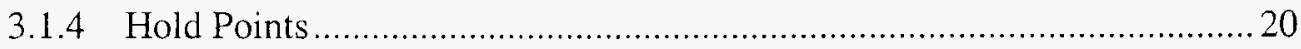

3.2 List Types of CONTAMINANTS OF POTENTIAL CONCERN AND AFFECTED MEDIA . 20

3.3 IDENTIFY POTENTIAL SAMPLING APPROACHES AND APPROPRIATE

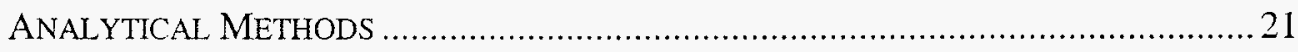

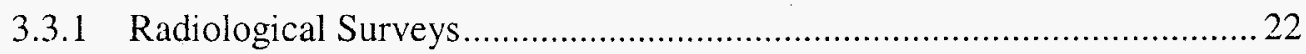

3.3.2 Radiological Field Screening .......................................................... 22

4.0 DEFINE THE STUDY BOUNDARIES (STEP 4) .............................................. 23

4.1 DeFINE THE GEOGRAPHIC AREAS OF THE FIELd INVESTIGATION ...........................23

4.1.1 Define the Geographic Area Within Which all Decisions Must Apply ....23

4.1.2 Specify the Characteristics that Define the Population of Interest ............ 23

4.2 DeFINE THE TIME FramE OF THE Decision.................................................... 23

4.2.1 Determine the Time Frame to Which the Study Data Apply ................... 23

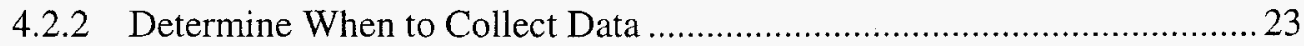

4.2.3 Define Relevant Time Constraints ......................................................... 23 


\section{TABLE OF CONTENTS (continued)}

4.3 DENTIFy ANy PRactical Constraints on Data Collection ..........................24

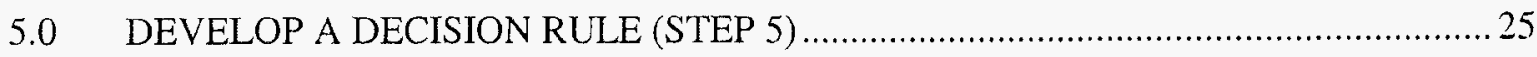

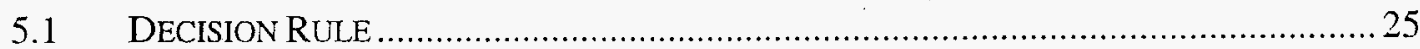

5.2 SPECIFY THE ACTION LEVEL OR PRELIMINARY ACTION LEVEL FOR THE DECISION 25

6.0 SPECIFY TOLERABLE LIMITS ON DECISION ERRORS (STEP 6) ..........................27

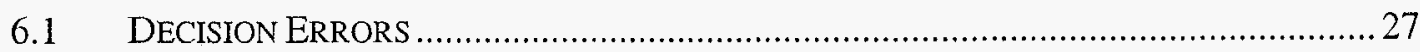

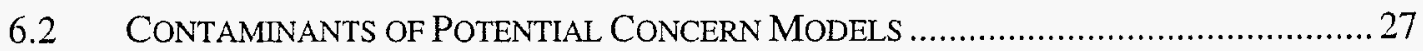

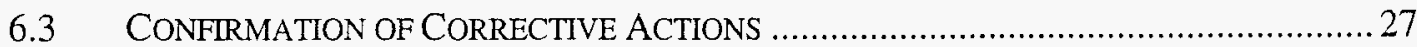

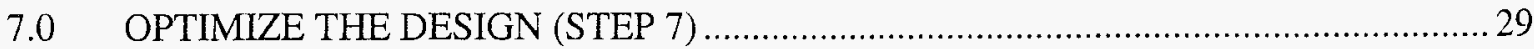

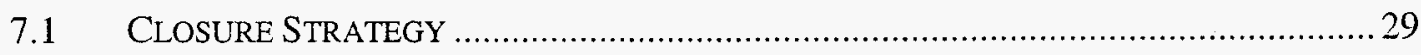

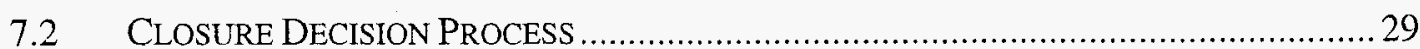

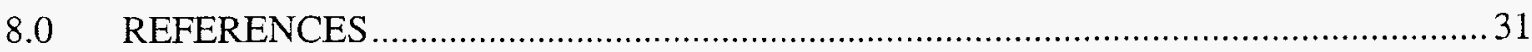




\section{ACRONYMS AND ABBREVIATIONS}

\begin{tabular}{|c|c|}
\hline $\mathrm{ACM}$ & Asbestos Containing Materials \\
\hline ALLW & Asbestiform Low-Level Waste \\
\hline $\mathrm{BN}$ & Bechtel Nevada \\
\hline CAS & Corrective Action Site \\
\hline CAU & Corrective Action Unit \\
\hline $\mathrm{COC}$ & Contaminants of concern \\
\hline $\mathrm{COPC}$ & Contaminants of potential concern \\
\hline CSM & Conceptual Site Model \\
\hline $\mathrm{D} \& \mathrm{D}$ & Deactivation and Decommissioning \\
\hline DOE & U.S. Department of Energy \\
\hline $\mathrm{DOE} / \mathrm{NV}$ & U.S. Department of Energy, Nevada Operations Office \\
\hline DQO & Data Quality Objective(s) \\
\hline EPA & U.S. Environmental Protection Agency \\
\hline FFACO & Federal Facility Agreement and Consent Order \\
\hline $\mathrm{ft}$ & foot (feet) \\
\hline $\mathrm{ft}^{2}$ & square feet \\
\hline $\mathrm{ft}^{3}$ & cubic feet \\
\hline FY & fiscal year \\
\hline gal & gallons \\
\hline HVAC & heating, ventilation, and air conditioning \\
\hline 1 & liter \\
\hline $\mathrm{m}$ & meter(s) \\
\hline $\mathrm{m}^{2}$ & square meters \\
\hline $\mathrm{m}^{3}$ & cubic meters \\
\hline NDEP & Nevada Division of Environmental Protection \\
\hline \multirow[t]{2}{*}{ NNSA/NSO } & U.S. Department of Energy, National Nuclear Security Administration Nevada \\
\hline & Site Office \\
\hline NTS & Nevada Test Site \\
\hline PCB & polychlorinated biphenyls \\
\hline QA/QC & Quality Assurance/Quality Control \\
\hline SAFER & Streamlined Approach for Environmental Restoration \\
\hline
\end{tabular}


SAFER PLAN - CAU 115

Section: Appendix A.1

Revision: 1

Date: December 2004

\section{ACRONYMS AND ABBREVIATIONS (continued)}

TCA Test Cell A

TCLP Toxicity Characterization Leaching Procedure

XRF X-Ray Fluorescence 
SAFER PLAN - CAU 115

Section: Appendix A.I

Revision: 1

Date: December 2004

\section{THIS PAGE INTENTIONALLY LEFT BLANK}

A. $1-8$ 


\section{APPENDIX A.1 \\ DATA QUALITY OBJECTIVES FOR CORRECTIVE ACTION UNIT 115: AREA 25 TEST CELL A FACILITY, NEVADA TEST SITE, NEVADA}

\section{Presentation of Known Data Related to Corrective Action Unit 115}

The information presented in this worksheet is based on historical data generated from preliminary assessment activities for Corrective Action Unit (CAU) 115: Test Cell A (TCA) Facility at the Nevada Test Site (NTS). The Data Quality Objective (DQO) worksheet follows the U.S. Environmental Protection Agency (EPA) DQO guidance outline (EPA, 2000b). The steps systematically build on the data acquired during preliminary assessment work and background research. Copies of the preliminary assessment work are retained in the CAU 115 project files.

Members of the Planning Team (* indicates members present at the DQO meeting):

1. U.S. Department of Energy, National Nuclear Security Agency Nevada Site Office (NNSA/NSO) Janet Appenzeller-Wing*

Sabine Curtis*

2. Bechtel Nevada (BN)

Jeff Smith

Pat Morris*

Dave Madsen*

Andrew Lacey*

Brian Konrad*

William Templeton

3. Nevada Division of Environmental Protection (NDEP)

John Wong*

4. Core Decision Team

Janet Appenzeller-Wing (NNSA/NSO)*

Sabine Curtis (NNSA/NSO)*

Pat Morris (BN)*

5. Primary Decision Makers

Janet Appenzeller- Wing (NNSA/NSO)*

Sabine Curtis (NNSA/NSO)* 


\subsection{STATE THE PROBLEM (STEP 1)}

Concisely describe the problem to be studied. Review prior studies and existing information to gain a sufficient understanding to define the problem.

The general location, nature, and extent of the CAU 115 corrective action sites (CASs) are understood; however, additional information is needed to verify that the existing information is adequate, confirm the existence of contamination and/or waste, and/or verify previously completed cleanup activities. Information will be used to close these CASs under the Streamlined Approach to Environmental Restoration (SAFER) process.

\subsection{CAS SPECIFIC INFORMATION}

CAU 115 is located on F Road in Area 25 of the NTS, approximately 87 miles north of Las Vegas, Nevada. It is comprised of four CASs:

- CAS 25-41-04, TCA Facility (Including Buildings 3113, 3113A, and 3113B)

- CAS 25-99-04 Asbestos Wrapped Piping (Exterior)

- CAS 25-99-05 Asbestos Wrapped Piping (Interior)

- CAS 25-99-06 Asbestos Wrapped Piping (Roof)

The following sections describe the CASs in CAU 115 and the conceptual site models (CSMs) that apply to each CAS.

\subsubsection{CAS 25-41-04, Test Cell A Facility}

CAU 115, TCA Facility, includes Buildings 3130, 3130-A, and 3130-B. Buildings 3113, 3113A, and $3113 \mathrm{~B}$ are constructed of steel-reinforced concrete. Total floor space of all three buildings is estimated to be 355 square meters $\left(\mathrm{m}^{2}\right)\left(3,821\right.$ square feet $\left.\left[\mathrm{ft}^{2}\right]\right)$. Building 3113 was constructed in 1958 and consists of two floors totaling $146 \mathrm{~m}^{2}\left(1,573 \mathrm{ft}^{2}\right)$. The first floor has approximately $111 \mathrm{~m}^{2}\left(1,200 \mathrm{ft}^{2}\right)$ and consists of an instrument room, equipment room, and the N-3 and N-4 rooms. The second floor consists of the Penthouse and Neutronics room. Building 3113A was constructed in 1961 and is approximately $139 \mathrm{~m}^{2}\left(1,500 \mathrm{ft}^{2}\right)$. This structure consists of the Flow Control Room. Building $3113 \mathrm{~B}$ contains approximately $69 \mathrm{~m}^{2}\left(748 \mathrm{ft}^{2}\right)$ and consists of restrooms and a computer storage room. Building 3130 is constructed of steel I-beams and corrugated metal panels. The TCA Facility was used to conduct the initial tests of nuclear rockets developed under Project Rover in Area 25 of the NTS. The NTS is located approximately 62 miles north of Las Vegas, Nevada.

The Preliminary Assessment (IT/LV, 1999) detailed the historical operations. From 1959 to 1966, the Kiwi, Nerva, and Phoebus reactors were tested at the TCA Facility. The reactors operated by fission of highly enriched Uranium-235 (U-235). The energy created by the fission of U-235 was absorbed by pumping hydrogen gas through the reactor where it was heated to 4,000 degrees Fahrenheit. The heated hydrogen was then exhausted through a nozzle at very 
SAFER PLAN - CAU 115

Section: Appendix A.I

Revision: 1

Date: December 2004

high velocities to produce thrust. After exiting the nozzle, the hydrogen gas was ignited, producing water vapor. The reactors were mounted on railcars and fired upward. Test runs ranged from several seconds to about one hour.

Testing resulted in the facility being contaminated with fission products and fuel particles. Most of the contamination consisted of isotopes with relatively short half-lives, and has since decayed away. The remaining isotopes of primary concern include Cobalt-60 (Co-60), Cesium-137 (Cs-137), and Strontium-90 (Sr-90). Additional isotopes that may be present in lesser abundance include Uranium-234 (U-234), -235 (U-235), -236 (U-236), -238 (U-238), and Plutonium-239 (Pu-239) and Plutonium-240 (Pu-240).

The TCA Facility was deactivated in 1966 and placed into long-term mothball status in 1973. The facility, excluding the roof, was successfully decontaminated in 1978 (International Technologies Las Vegas [ITLV], 1999). Since January 1999, the TCA Facility has been maintained under the NTS Deactivation and Decommissioning (D\&D) Surveillance \& Maintenance program.

The TCA facility contains the following known hazardous materials: lead, mercury, and asbestos containing materials (ACM) which are discussed further in the following sections. Suspected ACM building materials were sampled in November 2003 (Appendix A.4). Approximately 1,200 linear feet of ACM-insulated piping and an unspecified volume of $A C M$ in the form of mastic sealant were identified within the TCA Facility.

\subsubsection{CAS 25-99-04, Asbestos Wrapped Piping (Exterior)}

This CAS includes asbestos-wrapped piping located outside of the facility below the roofline. Specific small diameter piping sections covered with asbestos-containing insulation materials have been identified. These pipes and selected electrical cables are covered with silver-colored metallic foil. Previous analysis showed the foil to contain lead at a concentration of $1,000 \mathrm{mg} / \mathrm{kg}$ (ITLV, 1999), however, the X-Ray Fluorescence (XRF) field screening did not confirm these results. Samples of the silver-colored metallic foil covering the ACM insulation were collected and analyzed for toxicity characterization leading procedure (TCLP) lead.

\subsubsection{CAS 25-99-05, Asbestos Wrapped Piping (Interior)}

This CAS addresses a section of ducting in Building 3113A. The ducting is wrapped with asbestos-containing insulation materials and is not radiologically impacted.

\subsubsection{CAS 25-99-06, Asbestos Wrapped Piping (Roof)}

The mastic roofing sealant is located around roof penetrations on Buildings 3130 and $3130-\mathrm{A}$. This material was previously analyzed and determined to contain non-friable ACM at greater than one percent. The material was surveyed and confirmed to be radiologically impacted. The roofing material will be removed by qualified Asbestos Workers and containerized in 208-liter ( $l$ ) (55-gallon [gal]) steel drums. The waste will be managed as non-radiological Asbestiform Low-Level Waste (ALLW), as specified in Section 5.2.2, "Asbestiform Low-Level Waste."

\section{A.1-11}


SAFER PLAN - CAU 115

Section: Appendix A.1

Revision: 1

Date: December 2004

\subsection{DEVELOP/REFINE THE CONCEPTUAL MODEL}

The available information from which the conceptual model is based was derived from site process knowledge, historical background information, site analysis, and personnel interviews regarding historical activities related to the CASs. The conceptual side model is presented below.

\subsubsection{Test Cell A Deactivation and Decommissioning Conceptual Site Model}

\subsubsection{Radioactive Contamination}

The types and quantities of radiological contamination generated by the tests conducted at CAU 115 are well documented. Considerable amounts of information on the operation of the facility are presented in published literature and historical records. Available information includes the types of reactors, type of fuel used, power levels, run times, effluent, and results of past decontamination efforts. In addition, interviews with past workers were conducted to provide information on how facility operations were conducted and the known contaminants of concern (COC) produced during testing. Radiological contamination information is documented in the process knowledge of the facility.

Radiologically impacted areas of the facility are limited to the reactor pad, Building 3130 , the shielding wall of Building 3113 , the concrete pad and cryogenic gas transfer pipes on the south side of $3113 \mathrm{~A}$, the roofs of Buildings 3113 and $3113 \mathrm{~A}$, and the floor drain in Room 1.

Limited sections of piping and electrical conduit are embedded within the concrete walls and floors of the structure. Surveys indicate that these areas are slightly radiologically impacted (Appendix A.3). Heating, ventilation, and air conditioning (HVAC) systems are also present in portions of the facility. Preliminary surveys of a limited portion of the HVAC systems did not detect any radiological contamination (radiological contamination in the remaining areas was sporadic and of low activities).

Subsurface radiological soil contamination exists surrounding the facility. However, soil remediation is not included in the scope for CAU 115. Analytical results from three surface soil samples collected in October 2003 did not indicate any significant contamination.

\subsubsection{Chemical Contamination}

\subsection{Polychlorinated Biphenyls}

Analytical results confirm that polychlorinated biphenyls (PCBs) are present in fluorescent light ballasts and in the paint covering the walls and floors of Buildings 3113 and 3113A. The PCB concentrations range from 17 to 1,600 milligrams per kilogram $(\mathrm{mg} / \mathrm{kg})$. The TCLP lechate analysis was completed on two samples of paint. Results confirm that the PCB paint did not leach above regulatory guidelines. In its current state, the PCB paint does not pose a threat to human health or the environment. Applied dried paints, classified as PCB Bulk Product Waste 
SAFER PLAN - CAU 115

Section: Appendix A.1

Revision: 1

Date: December 2004

under Title 40 Code of Federal Regulations (CFR) 761.50 (b) 7 (EPA, 1996d), will be disposed of in a on-site disposal facility as demolition debris without treatment.

Analytical results confirm that $\mathrm{PCBs}$ are not present in the lubricating fluids known to be present at the TCA Facility and the main transformer that provided power to the facility was inspected and determined not to contain PCB oil.

\subsubsection{1 $\underline{\text { Lead }}$}

Hazardous materials present at the TCA Facility are limited to solid lead. Lead is present in shielding doors, as lead bricks, and as lead plate. Field screening results using an XRF instrument confirm that lead is not present in the paint.

The Preliminary Assessment reported that lead alloy foil is present around the ACM insulation that covers the majority of piping and electrical cable at the facility. However, field screening using an XRF instrument did not confirm these observations.

Solid lead was confirmed by visual observation. Lead is present as plate, bricks, and wool. Radiological surveys confirm that the majority of lead is not radiologically impacted. Lead bricks in the wall on as shielding in the reactor wall penetrations are shown in Figure 10.

\subsection{Asbestos Containing Materials}

The presence of ACM was confirmed by laboratory analysis. Non-friable ACM is present in the mastic roofing sealant located around roof penetrations on Buildings 3113 and 3113A. Friable ACM is present as pipe and HVAC duct insulation and around electrical cable. These areas of asbestos have been identified by CAS below and are identified by CAS:

- CAS 25-99-04 Asbestos wrapped pipes (exterior)

- CAS 25-99-05 Asbestos wrapped pipes (interior)

- CAS 25-99-06 Asbestos wrapped pipes (roof)

Suspected ACM building materials were sampled in November 2003 (Appendix A.4).

Approximately 1,200 linear feet of ACM-insulated piping and an unspecified volume of ACM in the form of mastic sealant were identified within the TCA Facility.

Most of the ACM insulated pipe and electrical cables are covered with silver-colored metallic foil. Previous analysis showed the foil to contain lead at a concentration of $1,000 \mathrm{mg} / \mathrm{kg}$ (ITLV, 1999). However, XRF field screening did not confirm these results. Samples of the silver foil covering the ACM insulation were collected and analyzed for TCLP lead.

The mastic roofing sealant is located around roof penetrations on Buildings 3130 and $3130-\mathrm{A}$. This material was previously analyzed and determined to contain non-friable ACM at greater than 1 percent. The material was surveyed and confirmed to be radiologically impacted. The 
roofing material will be removed by qualified Asbestos Workers and will be containerized in 208-L (55-gal) steel drums. The waste will be managed as non-radiological ALLW, as specified in Section 5.2.2, "Asbestiform Low-Level Waste."

\subsection{Mercury}

Mercury is only present in fluorescent light bulbs. No other mercury-containing items have been observed in the TCA Facility. All fluorescent light bulbs will be removed prior to demolition and managed appropriately. Possible mercury containing items and instrumentation will be identified for sampling. A representative item will be removed and analyzed for mercury and other hazardous constituents.

\subsection{Secondary Conceptual Site Models for CoRrective ACTION UNIT 115}

Based on the extensive available historical information and process knowledge, confirmed by site inspections and walkdowns, the initial CSM is substantiated and additional CSMs are not needed.

\subsection{Potential Hold Points}

During closure activities, certain conditions affecting the project schedule and budget may require decisions prior to continuing work. Primary hold/decision points that may occur during the CAU 115 SAFER process have been identified and include the review of data for the selection of potential site locations for further investigation and the review of analytical data.

During closure activities, certain conditions affecting the project schedule and budget may require decisions to be made prior to continuing work. If at any time during the course of closure activities a hold point is reached, the NNSA will obtain consensus with the NDEP prior to beginning the next phase of closure activities.

The D\&D Project Team will anticipate and minimize the necessary hold points. By establishing specific alternative actions, disruptions to the project schedule will be minimized. Primary hold/decision points that may occur during the CAU 115 SAFER process have been identified and include the review of data for the selection of potential site locations for further investigation and the review of analytical data.

Specific potential hold points include the following:

- $\quad$ Mitigation of safety hazards

- Verification of removal of hazardous materials

- Decontamination of reactor concrete pad and impacted concrete surfaces.

- Demolition and disposal of Buildings 3113, 3113A, 3113B, and 3130

- $\quad$ Final radiologically survey to free-release remaining concrete slab. 
In addition to the previously discussed hold/decision points, work may be temporarily suspended until the issue can be satisfactorily resolved if any of the following unexpected conditions occur:

- Conditions outside the scope of work are encountered

- Radiological screening yields results which require an upgrade in procedures to continue survey work in specific areas

- $\quad$ Elevated levels of additional COPCs are found that were not originally identified as being present at the sites

- Unexpected conditions including waste and/or contamination are encountered

- $\quad$ Out-of-scope work activities are required due to the detection of other COC that would require re-evaluating a disposal pathway, such as with hazardous or low level waste

- Unsafe conditions or work practices posing a threat to personnel, equipment, or the environment, not originally documented in the Site Specific Health and Safety Plan, are encountered

Other technical factors are encountered that require the preparation of a Record of Technical Change to the approved SAFER Plan. 
SAFER PLAN - CAU 115

Section: Appendix A:1

Revision: 1

Date: December 2004

\section{THIS PAGE INTENTIONALLY LEFT BLANK}

A.1-16 


\subsection{IDENTIFY THE DECISION (STEP 2)}

Identify what questions the study will attempt to resolve and what actions may result.

\subsection{DEVELOP DECISION STATEMENTS}

A SAFER Plan will be prepared based on the currently available process knowledge, historical information, geophysical data, and radiological survey data. Sites where the general location and nature of debris/contamination are known include the following CASs:

- CAS 25-41-04, TCA Facility (including Buildings 3113, 3113A, and 3113B)

- CAS 25-99-04 Asbestos Wrapped Piping (Exterior)

- CAS 25-99-05 Asbestos Wrapped Piping (Interior)

- CAS 25-99-06 Asbestos Wrapped Piping (Roof)

CASs with chemical (hazardous) or radiologically impacted materials will be clean closed by decontamination or removal of the impacted material. Clean closure will be verified by conducting a radiological survey on the remaining concrete pad to confirm all radioactive and chemical contamination has been removed.

\subsection{DECISION STATEMENTS}

Decision I: Is waste present and/or is contamination present above action levels within a CAS? A contaminant of potential concern (COPC) that is detected at a concentration exceeding action levels will be considered a COC. A COC is defined as a site-related constituent that exceeds the screening criteria or is detected during surface radiological surveys.

Decision II: If waste is present and/or contamination is present above action levels or screening levels, has the lateral and vertical extent been determined, and is it technically feasible to remove the waste/contamination? (If technically feasible, all CASs containing waste/contamination will be clean closed.) Hazardous materials will be removed and preliminary radiological surveys will determine the location and extent of contamination.

\subsection{Alternative ACtions to THE DECISION}

If a COPC is not present, further assessment of that COPC in the CAS is not required. If a COC is present, resolve Decision II. The alternative for Decision II is: "If the extent and migration of a COC is defined in both the lateral and vertical directions, further assessment of the CAS is not required. If the extent of a COC is not defined, reevaluate site conditions and collect additional samples." 
SAFER PLAN - CAU 115

Section: Appendix A.1

Revision: 1

Date: December 2004

THIS PAGE INTENTIONALLY LEFT BLANK

A. $1-18$ 


\subsection{IDENTIFY THE INPUTS TO THE DECISION (STEP 3)}

Identify the information that needs to be obtained and the measurements that need to be taken to resolve the decision statement. This step identifies the information needed and sources of information, the basis for establishing action levels, and sampling and analysis methods that can meet the data requirements.

\subsection{IDENTIFY THE INFORMATION INPUTS NEEDED AND RESOLVE THE DECISION}

In order to confirm the site CSM and to determine the nature and extent of contamination, data must be collected and analyzed following the following three criteria: 1) Survey data must be collected in areas containing impacted debris and/or contamination; 2) Samples will be collected from areas or items suspect to containing one of the identified COCs; and 3) The data must be adequate to detect COC.

Additional asbestos sampling will be performed prior to asbestos abatement activities beginning. Samples will be taken of electrical panels and components to identify if hazardous components exist. Verification samples will be taken as appropriate combined with final radiological surveys of the concrete pad.

In order to determine if a COC (e.g., radionuclides, asbestos, lead, PCBs, and mercury) is present at a particular CAS, sample data must be collected and analyzed by two criteria: 1) Samples must be collected in areas most likely to be contaminated; and 2) The requested analyses must be sufficient to detect any contamination present within the samples. Areas and specific items (i.e., piping, equipment, insulation, and penetrations) have been previously identified and sampled for their COC.

In order to confirm the extent of contamination, data must be collected and analyzed using a data collection method adequate to detect the COC. Samples will be collected based on radiological and geophysical surveys, field observations, and field screening results. Samples will be collected from areas expected not to be impacted by the COC so that the impacted area can be bounded and defined.

Biasing factors to support the determination of the nature and extent criteria include the following:

- Radiological surveys

- Geophysical surveys

- Documented process knowledge on source and location of release

- Field observations

- Experience and data from investigations of similar sites

- Professional judgment 
- Field screening results

\subsubsection{Quantitative Data}

Quantitative data measures the quantity or amount of a characteristic or component within the population of interest. These data require the highest level of quality assurance/quality control (QA/QC) in collection and measurement systems because the intended use of the data is to resolve primary decisions, and/or to verify that closure standards have been met. Laboratory analytical data are generally considered quantitative.

\subsubsection{Semi-Quantitative Data}

Semi-quantitative data indirectly measure the quantity or amount of a characteristic or component. Inferences are drawn about the quantity or amount of a characteristic or component because a correlation has been shown to exist between the indirect measurement and the results from a quantitative measurement. The $\mathrm{QA} / \mathrm{QC}$ requirements on semiquantitative collection and measurement systems are high but may not be as rigorous as a quantitative measurement system. Semiquantitative data contribute to decision making but are not sued alone to resolve primary decisions. Field-screening data are generally considered semiquantitative. The data are often used to guide investigations toward quantitative data collection.

\subsubsection{Qualitative Data}

Qualitative data identify or describe the characteristics or components of the population of interest. The QA/QC requirements are the least rigorous on data collection methods and measurement systems. The intended use of the data is for information purposes, to refine conceptual models, and to guide investigations rather than resolve primary decisions. This measurement of quality is typically assigned to historical information and data where QA/QC may be highly variable or not known. Professional judgment is often used to generate qualitative data.

\subsubsection{Hold Points}

Hold points will be designed into the investigation and closure activities for CAU 115. Hold points are designed to allow decision makers to review the existing data and decide which of the available options are most suitable. Hold points include the review of geophysical and radiological data and field observations for selection of targets for investigation, selection of corrective actions. The major hold points for this project have been identified and are discussed in Section 1.4.

\subsection{List TyPes of CONTAMinants OF Potential CONCERn AND AFFECTED MEDIA}

The following COCs have been identified as being associated with CAU 115:

- Lead 
- $\quad \mathrm{PCBs}$

- Asbestos

- Mercury

- Radionuclides

A list of identified COCs can be found in Table 1.

TABLE 1. CoRrective ACtion UnIT 115 WaSte AND CONTAMINANTS OF CONCERN DESCRIPTION

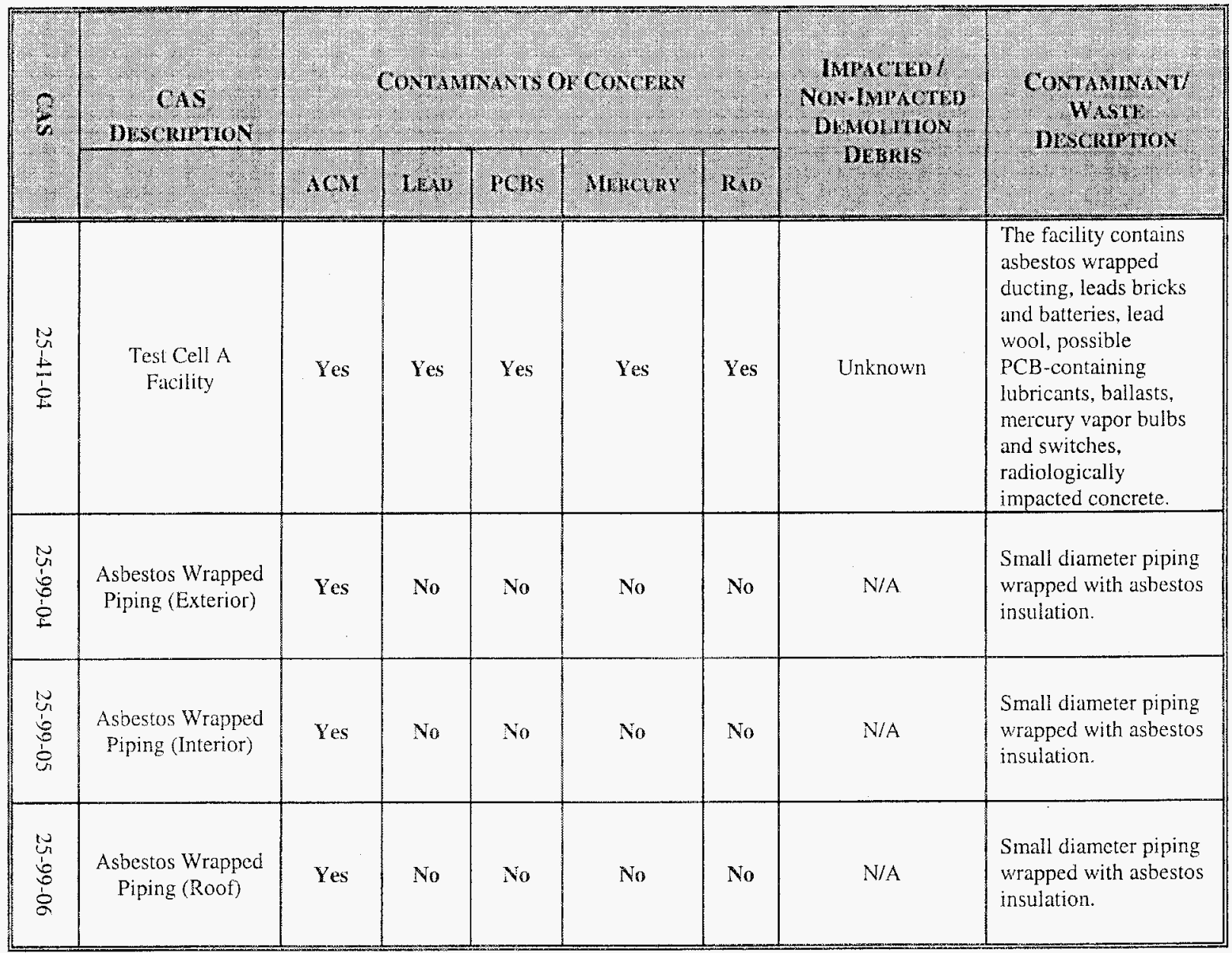

3.3

IDENTIFY POTENTIAL SAMPLING APPROACHES AND APPROPRIATE ANALYTICAL METHODS

The sampling techniques and analytical methods identified below will be used to resolve the decision rules and confirm the nature and extent of contamination at each CAS. 
Date: December 2004

\subsubsection{Radiological Surveys}

Radiological surveys will be used to determine the presence and lateral extent of radiological contamination. Preliminary surveys will determine the locations and extent of contamination on the reactor concrete pad. This information will be used to determine the appropriate equipment and controls to be used during decontamination.

\subsubsection{Radiological Field Screening}

Based on site conditions and available data, field-screening activities may be conducted for alpha and beta/gamma radiation. A handheld radiological survey instrument or method may be used, based on the possibility that radiologically contaminated soil/debris may be present. If determined appropriate, on-site gamma spectrometry may also be used to screen samples.

Field screening techniques may be used during the Decision I and II sampling activities. These field-screening techniques will provide semiquantitative data that can be used to guide potential confirmatory sampling and waste management activities. 


\subsection{DEFINE THE STUDY BOUNDARIES (STEP 4)}

Specify the time periods and spatial area to which decisions will apply. Determine when and where data should be collected. The purpose of this step is to define the target population of interest, specify the spatial and temporal features of that population that are pertinent for decision making, determine practical constraints on data collection, and define the scale of decision making relevant to target populations for Decision I and Decision II.

\subsection{DEFINE THE GEOGRAPHIC AREAS OF THE FIELD INVESTIGATION}

\subsubsection{Define the Geographic Area Within Which all Decisions Must Apply}

The CAU 115 limits are defined in the SAFER plan. The boundaries of the CAU and extent of the scope of work are outlined in this document.

\subsubsection{Specify the Characteristics that Define the Population of Interest}

The population of interest is the entire interior, exterior, and roof of the TCA Facility, including the concrete pad and moveable shed.

\subsection{DEFINE THE TIME FRAME OF THE DECISION}

\subsubsection{Determine the Time Frame to Which the Study Data Apply}

The study data should be relevant to the length of time allowed for by the SAFER process under the Federal Facility Agreement and Consent Order (FFACO) agreement. The decisions will be based on the documentation and data collection activities planned for 2004 and combined with the planned surveys to determine the proper recommendations for each of the CASs.

\subsubsection{Determine When to Collect Data}

Data collection activities are scheduled to begin in the fourth quarter of fiscal year (FY) 2004 and closure activities will be completed after approval of the final SAFER Plan. Data will be collected at times that meet the personnel and equipment availability constraints of the NTS, and at times when weather conditions that allow adequate site access and safe working conditions. A tentative schedule of activities for the completion of CAU 115 is presented in the SAFER Plan.

Radiological screening will be used to detect radiation above free-release criteria before any debris is removed off-site. There will be no other data collection, unless staining or other evidence of the presence of COPC or potential environmental impact is visibly detected and is determined to be related to CAU 115 .

\subsubsection{Define Relevant Time Constraints}

- The final SAFER Plan is due to the NNSA/NSO by September 19, 2004.

- The FFACO deadline for the SAFER Plan is September 30, 2004.

- $\quad$ Fieldwork is tentatively scheduled to begin during the first quarter of FY2005. 


\subsection{Inentify any Practical Constraints on Data Collection}

1. Approval of the SAFER Plan and the DQO process by the NDEP

2 Equipment access and mobility at the NTS

3. Meteorological events that may impact fieldwork activities

4. Health and safety of workers

5. Operational/Security issues at the NTS

6. Unforeseen conditions including unsafe working conditions or other factors 


\subsection{DEVELOP A DECISION RULE (STEP 5)}

Define the parameter of interest, specify the action level, and integrate the previous DQO inputs into a single statement that describes the logical basis for choosing among alternative actions. This step integrates outputs from the previous steps, with the inputs developed in this step into a decision rule ("If..., then...") statement. This decision rule describes the conditions under which possible alternative actions would be chosen.

\subsection{DECISION RULE}

Decision I: If waste is present and/or contamination is present above action levels, then the horizontal and vertical extent will be determined.

Decision II: $\quad$ If waste or contamination is present above action levels, and it is technically feasible to clean close the site, then the site will be clean closed.

If contamination is inconsistent with the CSM or extends beyond the identified CAS boundaries, work will be suspended and the investigation strategy will be reevaluated. If contamination is consistent with the CSM and is within CAS boundaries, the decision will be to define its extent.

\subsection{Specify the Action Level or Preliminary Action LeVel FOR THE DECISION}

Sufficient process knowledge and site surveys exist to support the CSMs. Asbestos, PCBs, lead, mercury, and radionuclides are the COCs within CAU 115. 
SAFER PLAN - CAU 115

Section: Appendix A.1

Revision: 1

Date: December 2004

\section{THIS PAGE INTENTIONALLY LEFT BLANK}

A.1-26 


\subsection{SPECIFY TOLERABLE LIMITS ON DECISION ERRORS (STEP 6)}

Define the decision makers' tolerable decision error rates based on a consideration of the consequences of making an incorrect decision.

\subsection{DECISION ERRORS}

If present, radiological contamination will be detected by radiological survey instrumentation, decontaminated, and removed to free release criteria as specified in DOE Order 5400.5. If radiological contamination is not present, then the remaining concrete foundation is already below free release criteria; therefore, decision error limits are not applicable.

\subsection{Contaminants of Potential Concern Models}

Models have been developed to characterize the COPCs for CASs within CAU 115. The models contain assumptions and statistical methodologies as appropriate to achieve the investigation/closure objectives. Table 1 summarizes the COPCs expected at CAU 115.

\subsection{CONFIRMATION OF CORRECTIVE ACTIONS}

The CAU 115 will be clean closed by removal of all hazardous materials, decontamination of radiologically impacted surfaces, and demolition of the structure(s). A confirmation radiological survey will be performed on the remaining concrete slab to determine that the remaining slab meets free release criteria as specified in DOE Order 5400.5, which is consistent with the Table 2 of the Yucca Mountain Project/NTS RadCon Manual. No soil excavation or removal will be performed as part of this closure activity. 
SAFER PLAN - CAU 115

Section: Appendix A.1

Revision: 1

Date: December 2004

\section{THIS PAGE INTENTIONALLY LEFT BLANK}

A. $1-28$ 


\subsection{OPTIMIZE THE DESIGN (STEP 7)}

Evaluate information from the previous steps and generate alternative data collection designs. Choose the most resource-effective design that meets all DQOs. This section presents an overview of the resource-effective strategy planned to obtain the data required to meet the project DQOs developed in previous steps. As additional data or information is obtained, this step will be reevaluated and refined, if necessary to reduce uncertainty and increase the confidence that the SAFER Corrective Action Investigation has met its intended goals.

\subsection{Closure Strategy}

The COCs will be addressed by the execution of the following closure strategy:

- $\quad$ Mitigate safety hazards

o Install portable lighting

o Hantavirus cleanup

o Site setup

- Removal of hazardous materials

o ACMs

o Lead (bricks, wool, etc.)

o Electrical components containing hazardous materials

o Radiologically impacted materials

o $\quad \mathrm{PCB}$ containing liquids (if present)

- Characterization and decontamination of reactor concrete pad and impacted concrete surfaces

- Demolition and disposal of Buildings 3113, 3113A, 3113B, and 3130 (Including exterior piping and exhaust stack). Dewar not included in the scope of this project.

- $\quad$ Final radiologically survey to free-release remaining concrete slab.

The attached "CAU 115 DQO Presentation" (Attachment A), which was presented to NNSA/NVO and the State of Nevada, summarizes this DQO document.

\subsection{Closure Decision Process}

Hold/decision points have been included in the process to allow critical data to be reviewed by the parties prior to proceeding with the closure activities. The three main hold points have been identified in the SAFER Plan. Only CASs determined to be associated with CAU 115 will be addressed within the corrective actions described in these DQOs. 
SAFER PLAN - CAU 115

Section: Appendix A.1

Revision: 1

Date: December 2004

THIS PAGE INTENTIONALLY LEFT BLANK

A. $1-30$ 


\subsection{REFERENCES}

EPA, see U.S. Environmental Protection Agency.

NDEP, see Nevada Division of Environmental Protection.

NNSA/NSO, see U.S. Department of Energy, National Nuclear Security Administration Nevada Site Office

U.S. Environmental Protection Agency. 2000a. Guidance for Choosing a Sampling Design for Environmental Data Collection (EPA QA/G-5), Office of Environmental Information, Washington, D.C.

U.S. Environmental Protection Agency. 2000b. Guidance for the Data Quality Objective Process, EPA QA/G-4, Washington, D.C.

U.S. Environmental Protection Agency. 2002. Region IX Preliminary Remediation Goals (PRGs), San Francisco, CA. 
SAFER PLAN - CAU 115

Section: Appendix A.1

Revision: 1

Date: December 2004

THIS PAGE INTENTIONALLY LEFT BLANK

A. $1-32$ 
Closure Report - CAU 115

Section: Appendix B

Revision: 0

Date: March 2006

\section{APPENDIX B}

\section{SAMPLE ANALYTICAL RESULTS}


Closure Report - CAU 115

Section: Appendix B

Revision: 0

Date: March 2006

THIS PAGE INTENTIONALLY LEFT BLANK 

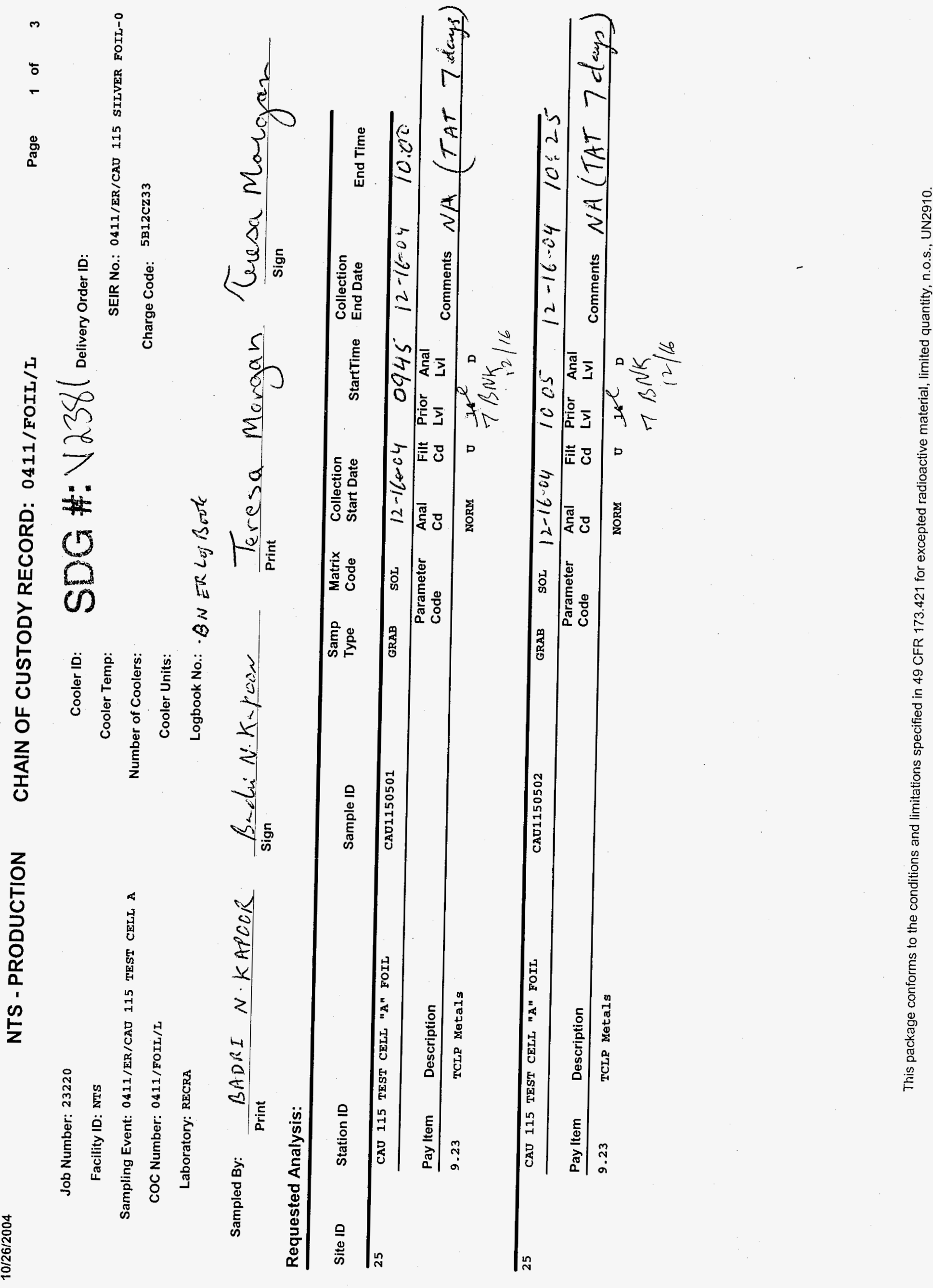
CLIENT: BECHTEL NEVADA V2381

WORK ORDER: 60052-001-001-0001-00

\begin{tabular}{|c|c|c|}
\hline SAMPLE & SITE ID & ANALYTE \\
\hline$====\approx==$ & 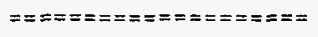 & 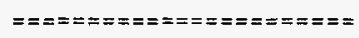 \\
\hline-003 & CAU115050101 & $\begin{array}{l}\text { Silver, TCLP Leachate } \\
\text { Arsenic, TCLP Leachate } \\
\text { Barium, TCLP Leachate } \\
\text { Cadmium, TCLP Leachate } \\
\text { Chromium, TCLP Leachate } \\
\text { Mercury, TCLP Leachate } \\
\text { Lead, TCLP Leachate } \\
\text { Selenium, TCLP Leachate }\end{array}$ \\
\hline-004 & CAU115050201 & $\begin{array}{l}\text { Silver, TCLP Leachate } \\
\text { Arsenic, TCLP Leachate } \\
\text { Barium, TCLP Leachate } \\
\text { Cadmium, TCLP Leachate } \\
\text { Chromium, TCLP Leachate } \\
\text { Mercury, TCLP Leachate } \\
\text { Lead, TCLP Leachate } \\
\text { Selenium, TCLP Leachate }\end{array}$ \\
\hline
\end{tabular}

LVL LOT \#: 0412L489

\begin{tabular}{|c|c|c|c|c|}
\hline & & & REPORTING & DILUTION \\
\hline RESULT & & UNITS & LIMIT & EACTOR \\
\hline$==\mathbf{=}=\mathbf{=}=$ & & $\Rightarrow=====$ & $\Rightarrow=== \pm==\equiv==$ & $=======$ = \\
\hline 6.0 & $\mathrm{u}$ & $\mathrm{UG} / \mathrm{L}$ & 6.0 & 6.0 \\
\hline 15.6 & $u$ & $\mathrm{UG} / \mathrm{L}$ & 15.6 & 6.0 \\
\hline 153 & & $U G / L$ & 2.4 & 6.0 \\
\hline 180 & & UG/L & 1.8 & 6.0 \\
\hline 6.2 & & UG/L & 4.8 & 6.0 \\
\hline 0.10 & $\mathrm{u}$ & $\mathrm{UG} / \mathrm{L}$ & 0.10 & 1.0 \\
\hline 783 & & UG / L & 13.2 & 6.0 \\
\hline 22.2 & $\mathrm{u}$ & UG/L & 22.2 & 6.0 \\
\hline 6.0 & $\mathrm{u}$ & UG/L & 5.0 & 6.0 \\
\hline 15.6 & $\mathbf{u}$ & $\mathrm{UG} / \mathrm{L}$ & 15.6 & 6.0 \\
\hline 217 & & $U G / L$ & 2.4 & 6.0 \\
\hline 9370 & & UG/L & 1.8 & 6.0 \\
\hline 11.0 & & UG $/ \mathrm{L}$ & 4.8 & 6.0 \\
\hline 0.10 & u & UG / L & 0.10 & 1.0 \\
\hline 528 & & $\mathrm{UG} / \mathrm{L}$ & 13.2 & 6.0 \\
\hline 22.2 & $\mathrm{u}$ & UG $/ L$ & 22.2 & 6.0 \\
\hline
\end{tabular}


Lionvilie Laboratory, Inc.

INORGANICS METHOD BLANI DATA SUMMAPY PAGE 12/28/04

CLIENT: BECHTEL NEVADA. V2331

WORK ORDER: 60052-001-001-0001-00

LVL LOT \#: O412L489

\begin{tabular}{|c|c|c|c|c|c|c|c|}
\hline & & & & & & REPORTING & DILUTION \\
\hline SAMPLE & SITE ID & ANALYTE & RESULT & & UNITS & LIMIT & FACTOR \\
\hline$======$ & 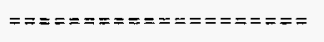 & = = = = = = = = = = = = = = = = = = = = = & $==\approx= \pm==$ & & $======$ & 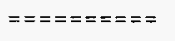 & $==\equiv=====$ \\
\hline \multirow[t]{7}{*}{ BLANK1. } & $04 \mathrm{~L} 0772-\mathrm{MB} 1$ & Silver, tCLP Leachate & 1.0 & $\mathrm{u}$ & UG /L & 1.0 & 1. 0 \\
\hline & & Arsenic, TCLP Leachate & 2.6 & u. & UG /L & 2.6 & 1.0 \\
\hline & & Barium, TCLP Leachate & 2.2 & & UG $/ \mathrm{L}$ & 0.40 & 1.0 \\
\hline & & Cadmium, TCLP Leachate & 0.30 & $\mathrm{u}$ & UG /L & 0.30 & 1.0 \\
\hline & & Chromium, TCLP Leachate & 1.9 & & UG $/ L$ & 0.80 & 1.0 \\
\hline & & Lead, TCLP Leachate & 2.2 & u & UG/L & 2.2 & 1.0 \\
\hline & & Selenium, TCLF Leachate & 3.7 & $u$ & UG /L & 3.7 & 1.0 \\
\hline \multirow[t]{7}{*}{ BLANNK2 } & $04 \mathrm{~L} 0772-\mathrm{ME} 2$ & Silver, TCLP Leachate & 6.0 & $\mathrm{u}$ & $\mathrm{UG} / \mathrm{L}$ & 6.0 & 6.0 \\
\hline & & Axsenic, TCLP Leachate & 15.6 & $\mathrm{u}$ & UG / L & 15.6 & 6.0 \\
\hline & & Barium, TCLP Leachate & 7.1 & & $\mathrm{UG} / \mathrm{L}$ & 2.4 & 6.0 \\
\hline & & Cadmium, TCLP Leachate & 1.8 & $\mathrm{u}$ & UG $/ \mathrm{L}$ & 1.8 & 6.0 \\
\hline & & Chromium, TCLP Leachate & 4.8 & $\mathrm{u}$ & UG/L & 4.8 & 6.0 \\
\hline & & Lead, TCLP Leachate & 13.2 & $u$ & $\mathrm{UG} / \mathrm{L}$ & 13.2 & 6.0 \\
\hline & & Selenium, TCLP Leachate & 22.2 & $\mathrm{u}$ & UG /L & 22.2 & 6.0 \\
\hline BLANIKI & $04 \mathrm{C} 0282-M B 1$ & Mercury, Total. & 0.10 & $u$ & $U G / L$ & 0.10 & 1.0 \\
\hline BLANIK2 & $04 \mathrm{C} 0282-M B 2$ & Mercury, TCLP Leachate & 0.10 & $\mathrm{u}$ & UG /L & 0.10 & 1.0 \\
\hline BLANK3 & $04 \mathrm{CO} 282-\mathrm{MB} 3$ & Mercury, TCLP Leachate & 0.10 & $u$ & $\mathrm{UG} / \mathrm{L}$ & 0.10 & 1.0 \\
\hline
\end{tabular}


Lionville Laboratory, Inc.

INORGÄNICS ACCURACY REPORT 12/28/04

CLIENT: BECHTEL NEVADA V2381

WORK ORDER: 60052-001-001-0001-00
LVL LOT \#: $0412 \mathrm{~L} 489$

\begin{tabular}{|c|c|c|c|c|c|}
\hline & SPIKED & INITIAL & SEIKED & & DILUTION \\
\hline NALYTE & SAMPLE & RESULI & AMOUNT & \%RECOV & FACTOR (SPIK) \\
\hline = = = = = = = = = = = = = = = = = = & $\Rightarrow=z== \pm=$ & $======$ & $=====$ & $====\equiv=z$ & $=\Sigma= \pm=\Sigma=\Sigma==$ \\
\hline ilver, TCLP Leachate & 4250 & $6.0 \mathrm{u}$ & 5000 & 85.0 & 6.0 \\
\hline rsenic, TCLP Leachate & 4200 & $15.6 \mathrm{u}$ & 5000 & 84.0 & 6.0 \\
\hline rium, TCLP Leachate & 80800 & 153 & 100000 & 80.6 & 6.0 \\
\hline admium, TCLP Leachate & 1010 & 180 & 1000 & 83.3 & 6.0 \\
\hline hromium, TCLP Leachat & 4230 & 6.2 & 5000 & 84.5 & 6.0 \\
\hline ercury, TCLP Leachate & 179 & $0.10 \mathrm{u}$ & 200 & 89.5 & 100 \\
\hline ad, TCLP Leachate & 4990 & 783 & 5000 & 84.1 & 6.0 \\
\hline lenium, TCLP Leachat & 860 & $22.2 \mathrm{u}$ & 1000 & 86.0 & 6.0 \\
\hline
\end{tabular}


Lionville Laboratory, Inc.

INORGANICS LABORATORY CONTROL STANDARDS REPORT 12/28/04

CLIENT: BECHTEL NEVADA V2381

WORT ORDER: 60052-001-001-0001-00

\begin{tabular}{|c|c|c|}
\hline SAMPLE & SITE ID & ANELYTE \\
\hline$======$ & = = = = = = = = = = = = = = = = = = & " \\
\hline LCS1 & $04 \mathrm{~L} 0772-\mathrm{LC} 1$ & $\begin{array}{l}\text { Silver, LCS } \\
\text { Arsenic, LCS } \\
\text { Barium, LCS } \\
\text { Cadmium, LCS } \\
\text { Chromium, LCS } \\
\text { Lead, LCS } \\
\text { Selenium, LCS }\end{array}$ \\
\hline LCS 1 & $04 C 0282-L C 1$ & Mercury, LCS \\
\hline
\end{tabular}

LVL LOT \#: 0412L489

$\begin{array}{crrr}\text { SPIKED } & \text { SPIKED } & & \\ \text { SAMPLE } & \text { AMOUNT } & \text { UNITS } & \text { \%RECOV } \\ ===== & ===== & ===== & ==== \\ 474 & 500 & \mathrm{UG} / \mathrm{L} & 94.8 \\ 8980 & 10000 & \mathrm{UG} / \mathrm{L} & 89.8 \\ 4970 & 5000 & \mathrm{UG} / \mathrm{L} & 99.4 \\ 232 & 250 & \mathrm{UG} / \mathrm{L} & 92.9 \\ 484 & 500 & \mathrm{UG} / \mathrm{L} & 96.9 \\ 2350 & 2500 & \mathrm{UG} / \mathrm{L} & 93.9 \\ 8510 & 10000 & \mathrm{UG} / \mathrm{L} & 85.1 \\ & & & 100.1\end{array}$




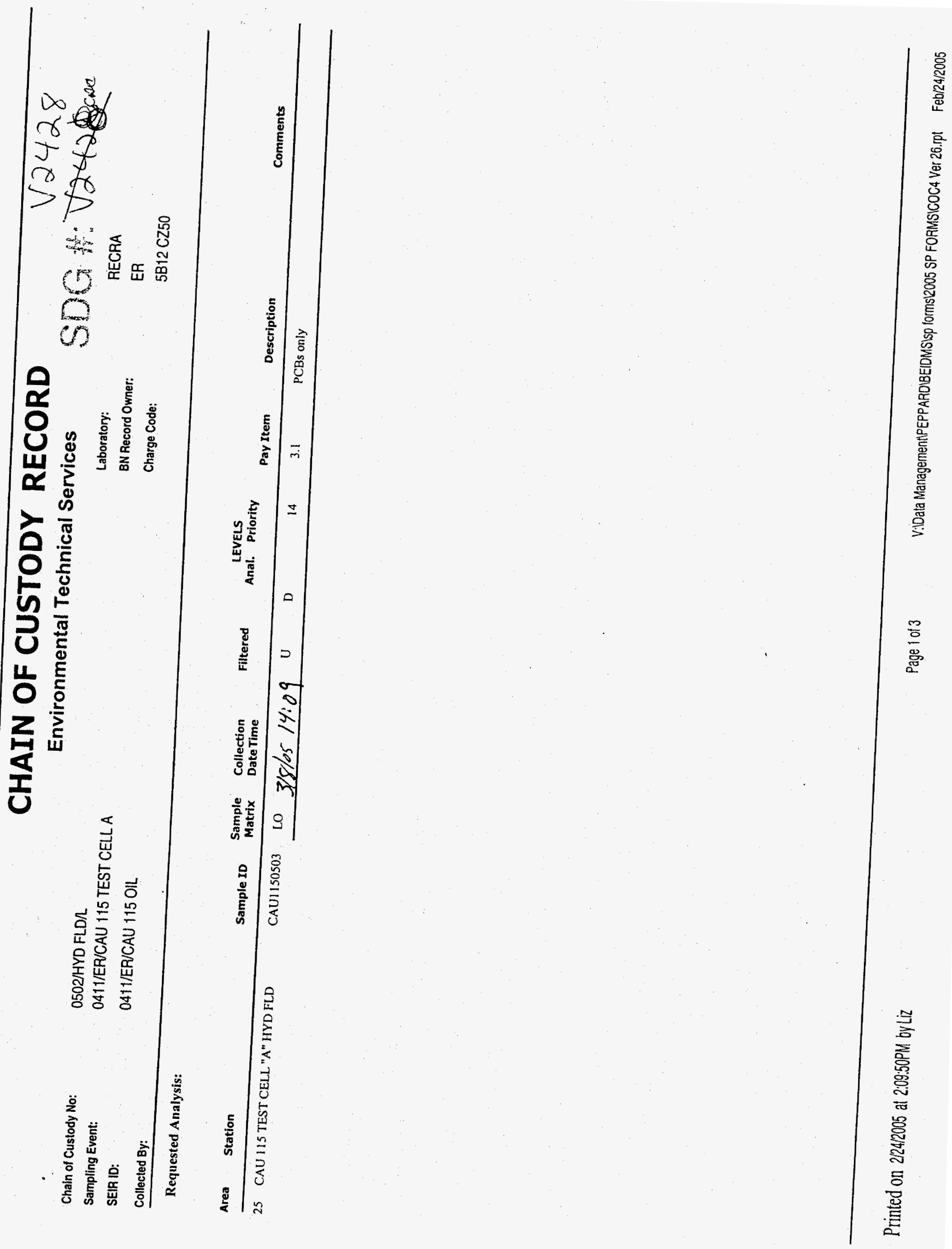




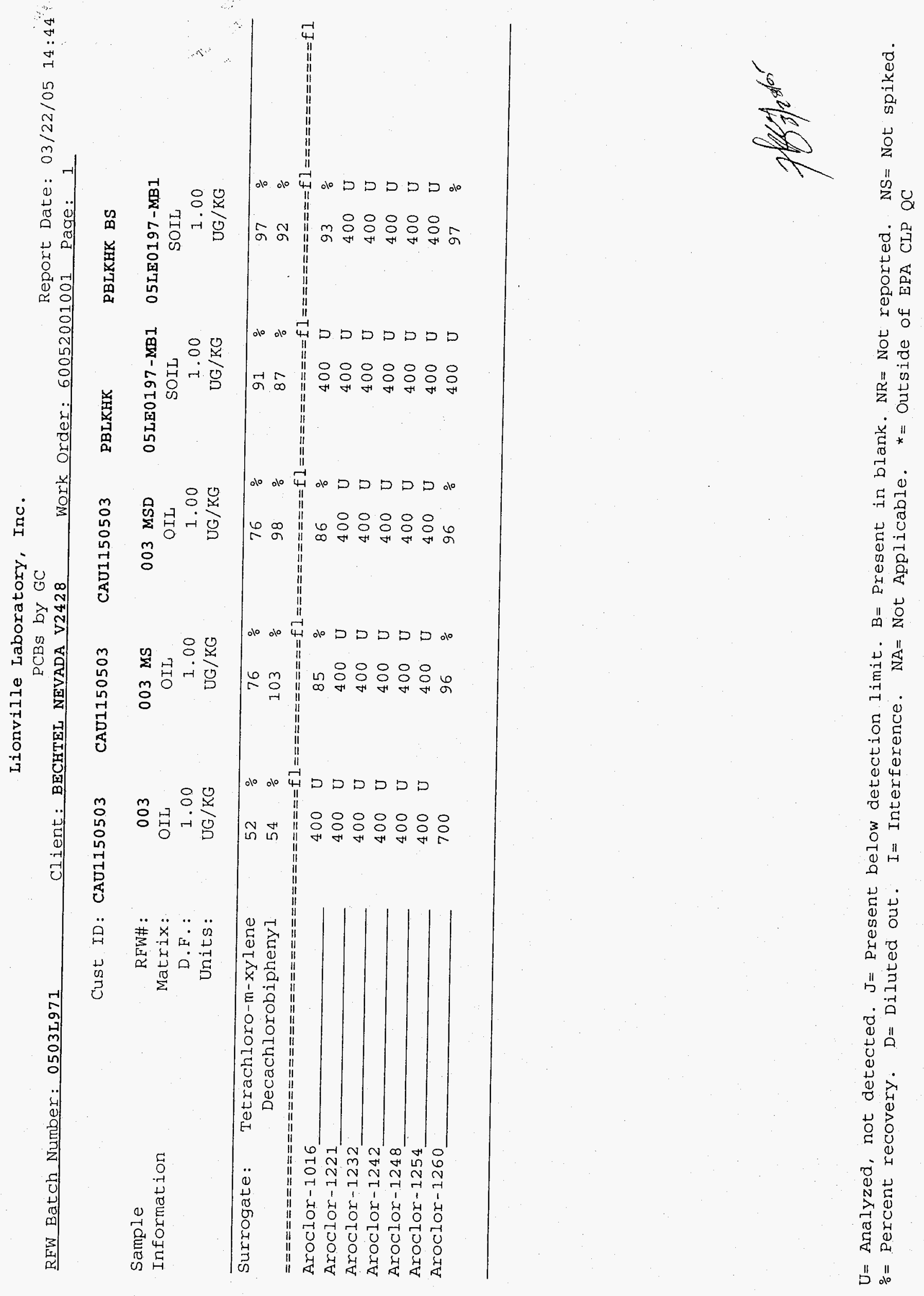


Lab Name: Lionville Labs, Inc.

Contract: $\quad \underline{0052-01-01}$

Case No.: BECHTEL NEVADA V2428

RFW Lot No.:

\begin{tabular}{|c|c|c|c|}
\hline & CLIENT & S1 & |OTHER \\
\hline & SAMPLE NO. & ( ) \# & $\mathrm{DCB}$ \\
\hline & 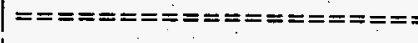 & $======0$ & $=======$ \\
\hline 01 & | CAU1150503 & 52 & 54 \\
\hline 02 & | CAU1150503MS & 76 & 103 \\
\hline 03 & CAU1150503MSD & 76 & 98 \\
\hline 04 & | PBLKHKLE0197-MBI & 91 & 87 \\
\hline 05 & PBLKHKLE0197-MB1 BS & 97 & 92 \\
\hline
\end{tabular}

ADVISORY

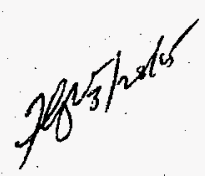

S1 ( ) = Tetrachloro-m-xylene

QC IIMITS

S2 $(\mathrm{DCB})=$ Decachlorobiphenyl

$(28-118)$

$(38-122)$

\# Column to be used to flag recovery values

* Values outside of QC limits

$D$ Surrogates diluted out 
SOIL PESTICIDE MATRIX SPIKE/MATRIX SPIKE DUPLICATE RECOVERY

Lab Name: Lionville Labs, Inc.

Case No.: BECHTEL NEVADA V2428

MATRIX Spike - Sample No.: CAU1150503
Contract: $\quad \underline{0052-01-01}$

RFW Lot No.: 0503L971-003

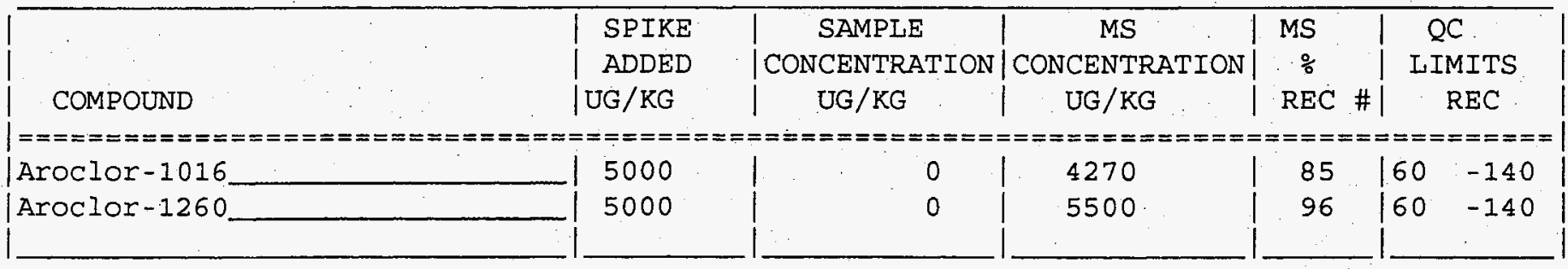

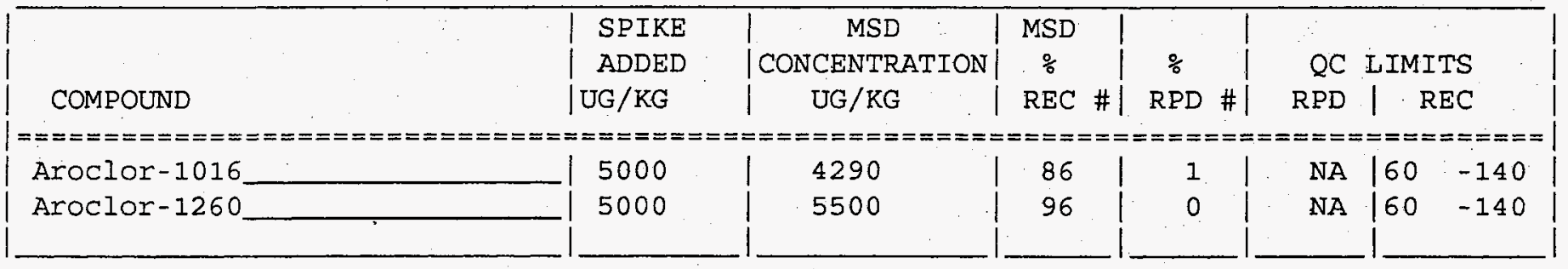

\# Column to be used to flag recovery and RPD values with an asterisk

* Values outside of QC limits

RPD: $\underline{0}$ out of $\underline{2}$ outside limits

Spike Recovery: 느 out of $\underline{4}$ outside limits

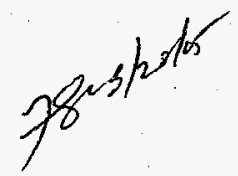

COMMENTS : 
$3 F$

SOIL PESTICIDE MATRIX SPIKE RECOVERY

Lab Name: Lionville Labs. Inc.

Contract: $\underline{0052-01-01}$

Case No.: BECHTEL NEVADA V2428

RFW Lot No.: 0503L971

MATRIX Spike - Sample No.: PBLKHKLE0197-MBI

Level: (low/med) LOW

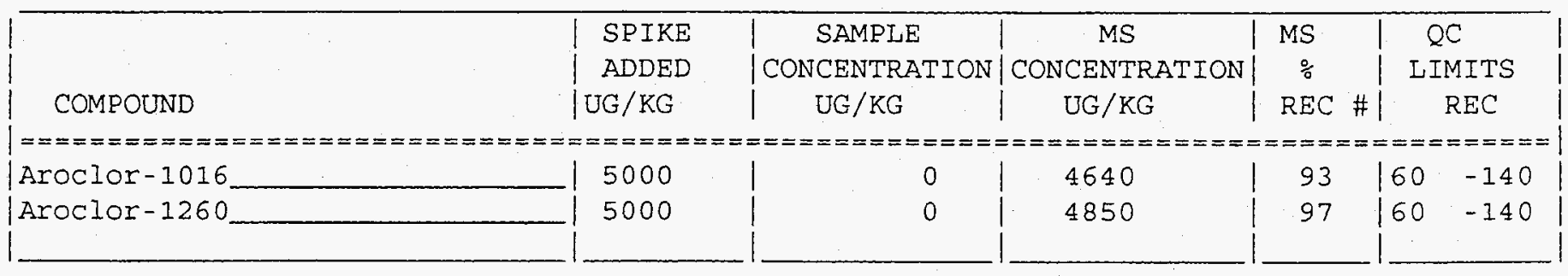

\# Column to be used to flag recovery value with an asterisk

* Values outside of QC limits

Spike Recovery: 으 out of $\underline{2}$ outside limits

COMMENTS :

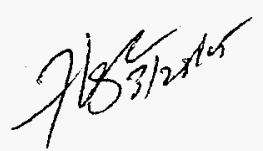


$4 D$

METHOD BLANK SUMMARY

Lab Name: Lionville Labs, Inc.

Case No.: BECHTEL NEVADA V2428

Lab Sample ID: 05LE0197-MB1

Matrix:(Soil/Water) SOIL

Date Extracted: $03 / 17 / 05$

Date Analyzed (1): 03/19/05

Instrument ID (1): 13
Contract: $60052-001-001-0001-00$

Lab File ID: BLKO8330.01

Level: (low/med) LOW

Extraction:(SepF/Cont/Sonc) DIL

Time Analyzed (1):

GC Column ID (1): $\underline{\text { RTX-CLP }}$

THIS METHOD BLANK APPLIES TO THE FOLLOWING SAMPLES, MS, AND MSD:

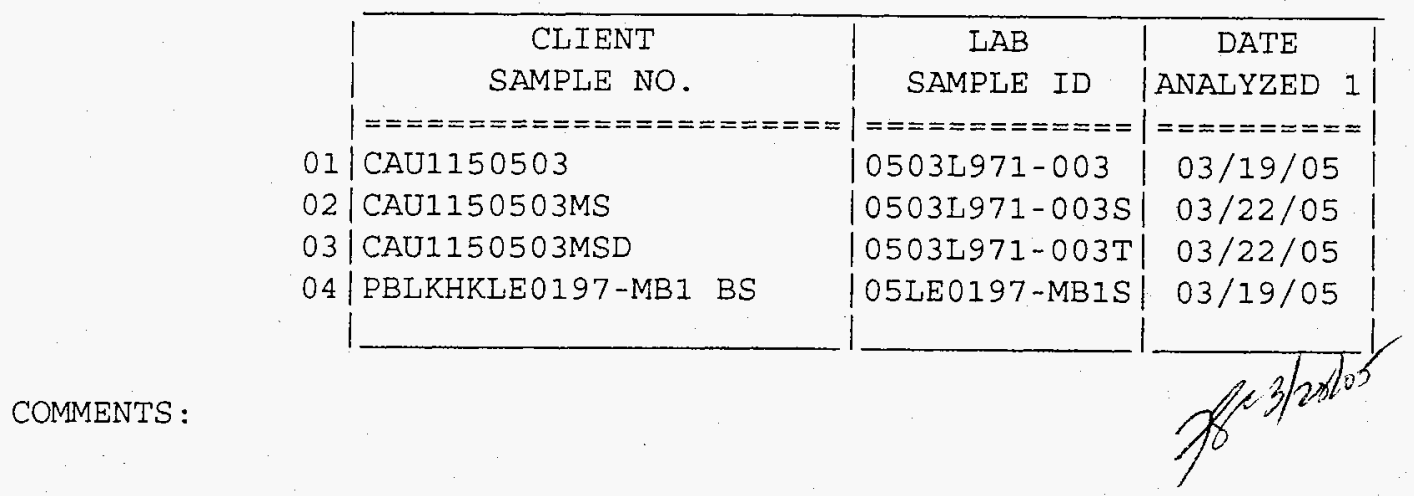

COMMENTS :

page 1 of 1

FORM IV 

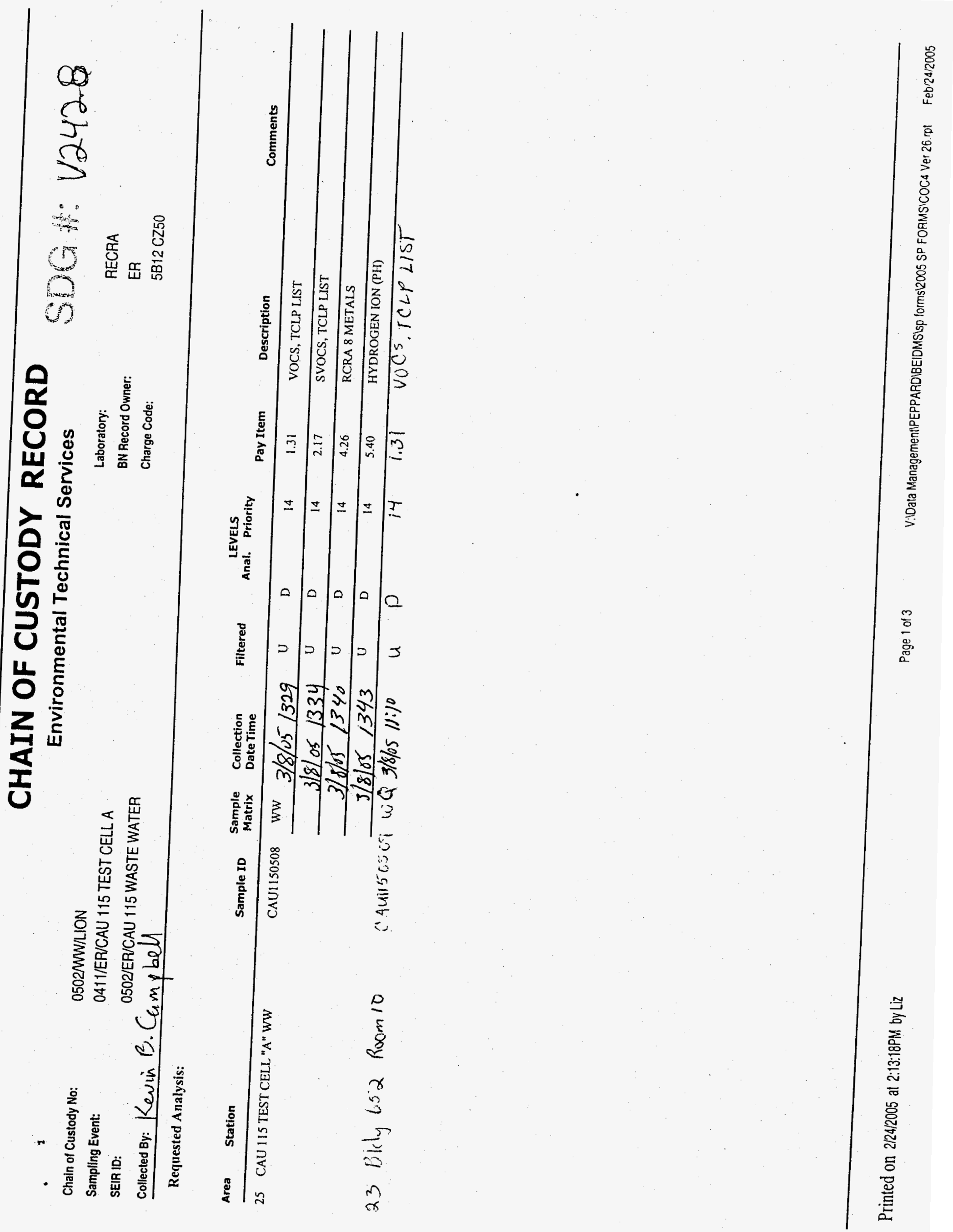
INORGANICS DATA SUMMARY REPORT 03/28/05

CLIENT: BECHTEL NEVADA V2428

WORK ORDER: 60052-001-001-0001-00

\begin{tabular}{|c|c|c|}
\hline SAMPLE & SITE ID & ANALYTE \\
\hline 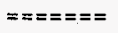 & 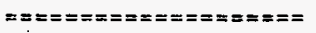 & 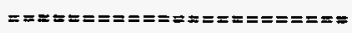 \\
\hline \multirow[t]{8}{*}{-001} & CAU1150508 & Silver, Total \\
\hline & & Arsenic, Total \\
\hline & & Barium, Total \\
\hline & & Cadmium, Total \\
\hline & & Chromium, Total \\
\hline & & Mercury, Total \\
\hline & & Lead, Total \\
\hline & & Selenium, Total \\
\hline
\end{tabular}

LVL LOT \# : 0503 L971

\begin{tabular}{|c|c|c|c|}
\hline & & REPORTING & DILUTION \\
\hline RESULT & UNITS & LIMIT & FACTOR \\
\hline$===\equiv=m=$ & $==x=x=$ & 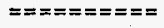 & $=======\pi$ \\
\hline 0.50 & $\mathrm{UG} / \mathrm{L}$ & 0.50 & 1.0 \\
\hline 3.4 & $U G / L$ & 2.9 & 1.0 \\
\hline 210 & UG $/ \mathrm{L}$ & 0.20 & 1.0 \\
\hline 0.40 & $\mathrm{UG} / \mathrm{L}$ & 0.40 & 1.0 \\
\hline 7.6 & $\mathrm{UG} / \mathrm{L}$ & 0.40 & 1.0 \\
\hline 0.10 & UG $/ L$ & 0.10 & 1.0 \\
\hline 1.9 & $\mathrm{UG} / \mathrm{L}$ & 1.9 & 1.0 \\
\hline 4.0 & UG/L & 4.0 & 2.0 \\
\hline
\end{tabular}


Lionville Laboratory, Inc.

INORGANICS METHOD BLANK DATA SUMMARY PAGE 03/28/05

CLIBNT: BECHTEL NEVADA V2428

WORK ORDER: 60052-001-001-0001-00

\begin{tabular}{|c|c|c|}
\hline SAMPLE & SITE ID & ANALYTE \\
\hline$=\approx=ニ \approx==$ & 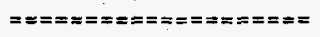 & 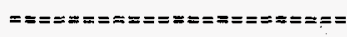 \\
\hline BLANK I & $05 L 0152-M B 1$ & $\begin{array}{l}\text { Silver, Total } \\
\text { Argenic, Total } \\
\text { Barium, Total } \\
\text { Cadmium, Total } \\
\text { Chromium, Total } \\
\text { Lead, Total } \\
\text { Selenium, Total }\end{array}$ \\
\hline BILANKI & $05 \mathrm{CO} 056-\mathrm{MB1}$ & Mercury, Total \\
\hline
\end{tabular}

LVL LOT \#: 0503L971

\begin{tabular}{|c|c|c|c|c|}
\hline & & & REPORTING & DILUTION \\
\hline RESULT & & UNITS & LIMIT & FACTOR \\
\hline$======$ & & $======$ & $== \pm====x=$ & $==== \pm===$ \\
\hline 0.50 & $u$ & $\mathrm{UG} / \mathrm{L}$ & 0.50 & 1.0 \\
\hline 2.9 & $\mathbf{u}$ & UG/L & 2.9 & 1.0 \\
\hline 0.51 & & UG/L & 0.20 & 1.0 \\
\hline 0.40 & $\mathrm{u}$ & $U G / L$ & 0.40 & 1.0 \\
\hline 0.40 & $\mathbf{u}$ & UG/L & 0.40 & 1.0 \\
\hline 1.9 & $\mathbf{u}$ & UG/L & 1.9 & 1.0 \\
\hline 4.0 & $\mathbf{u}$ & $\mathrm{UG} / \mathrm{L}$ & 4.0 & 1.0 \\
\hline 0.10 & $\mathbf{u}$ & UG $/ L$ & 0.10 & 1.0 \\
\hline
\end{tabular}


Lionville Laboratory, Inc.

INORGANICS ACCURACY REPORT 03/28/05

CLIENT: BECHTEL NEVADA V2428

WORK ORDER: 60052-001-001-0001-00
LVL LOT \#: 0503L971

\begin{tabular}{|c|c|c|c|c|c|c|c|}
\hline & & & SPIKED & INITIAL & SPIKED & & DILUTION \\
\hline SAMPLE & SITE ID & ANALYTE & SAMPLE & RESULT & AMOUNT & \% RECOV & FACTOR (SPK) \\
\hline$=======$ & 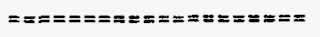 & 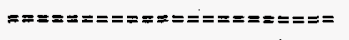 & $==2===\approx=$ & $=== \pm===$ & $== \pm===$ & $== \pm====$ & $=======\approx=0$ \\
\hline \multirow[t]{14}{*}{-001} & CAU1150508 & Silver, Total & 48.8 & $0.50 u$ & 50.0 & 97.6 & 1.0 \\
\hline & & Silver, Total MSD. & 48.6 & $0.50 u$ & 50.0 & 97.2 & 1.0 \\
\hline & $\cdot$ & Axsenic, Total & 1910 & 3.4 & 2000 & 95.1 & 1.0 \\
\hline & · & Arsenic, Total & 1900 & 3.4 & 2000 & 94.7 & 1.0 \\
\hline & & Barium, Total & 2150 & 210 & 2000 & 97.1 & 1.0 \\
\hline & & Barìum, Total & 2130 & 210 & 2000 & 96.1 & 1.0 \\
\hline & & Cadmium, Total & 46.8 & 0.404 & 50.0 & 93.6 & 1.0 \\
\hline & & Cadmium, Total & 46.5 & $0.40 \mathrm{u}$ & 50.0 & 93.0 & 1.0 \\
\hline & & Chromium, Total & 198 & 7.6 & 200 & 95.2 & 1.0 \\
\hline & & Chromium, Total MṢD & 197 & 7.6 & 200 & 94.5 & 1.0 \\
\hline & & Lead, Total & 478 & $1.9 \mathrm{u}$ & 500 & 95.7 & 1.0 \\
\hline & & Lead, Total MSD & 480 & $2.9 \mathrm{u}$ & 500 & 96.1 & 1.0 \\
\hline & & Selenium, Total & 1880 & $4.0 \mathrm{u}$ & 2000 & 93.9 & 1.0 \\
\hline & & Selenium, Total & 1880 & $4.0 \mathrm{u}$ & 2000 & 93.9 & 1.0 \\
\hline
\end{tabular}


Lionville Laboratory, Inc.

INORGANICS DUPLICATE SPIKE REPORT 03/28/05

CLIENT: BECHTEL NEVADA V2428

WORK ORDER: 60052-001-001-0001-00
LVL LOT \#: 0503L971

\begin{tabular}{|c|c|c|c|c|c|}
\hline \multirow[b]{2}{*}{ SAMPLE } & \multirow{2}{*}{ SITE ID } & \multirow[b]{2}{*}{ ANALYTE } & \multicolumn{3}{|c|}{ SPIKE\#1 SPIKB\#2 } \\
\hline & & & \$RECOV & \&RECOV & \&DIFF \\
\hline$=x===x=$ & 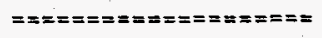 & 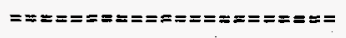 & $==\approx==\mathbf{=}$. & $==== \pm=$ & $=====0$ \\
\hline \multirow{7}{*}{-001} & CAU1150508 & Silver, Total & 97.6 & 97.2 & 0.41 \\
\hline & & Arsenic, Total & 95.1 & 94.7 & 0.41 \\
\hline & & Barium, Total & 97.1 & 96.1 & 1.0 \\
\hline & & Cadmium, Total & $93.6^{\circ}$ & 93.0 & 0.64 \\
\hline & & Chromium, Total & 95.2 & 94.5 & 0.74 \\
\hline & & Lead, Total & 95.7 & 96.1 & 0.40 \\
\hline & & Selenium, Total & 93.9 & 93.9 & 0.021 \\
\hline
\end{tabular}


Lionville Laboratory, Inc.

INORGANICS PRECISION REPORT 03/28/05

CLIENT: BECHTEL NEVADA V2428

WORK ORDER: 60052-001-001-0001-00
LVL LOT \# : 0503L971

\begin{tabular}{|c|c|c|}
\hline SAMPLE & SITE ID & ANALYTE \\
\hline 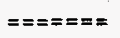 & 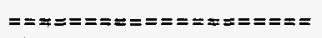 & 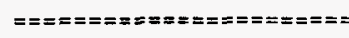 \\
\hline$-001 R E P$ & CAU1150508 & $\begin{array}{l}\text { Silver, Total } \\
\text { Arsenic, Total } \\
\text { Barium, Total } \\
\text { Cadmium, Total } \\
\text { Chromium, Total } \\
\text { Lead, Total } \\
\text { Selenium, Total }\end{array}$ \\
\hline
\end{tabular}

$\begin{array}{lcc}\text { INITIAL } & \\ \text { RESULT } & \text { REPLICATE } & \text { RQD } \\ ==== & ============ \\ 0.50 \mathrm{U} & 0.50 \mathrm{u} & \mathrm{NC} \\ 3.4 & 2.9 & 15.9 \\ 210 & 209 & 0.19 \\ 0.40 \mathrm{u} & 0.40 \mathrm{u} & \mathrm{NC} \\ 7.6 & 8.6 & 12.3 \\ 1.9 \mathrm{u} & 1.9 \mathrm{u} & \mathrm{NC} \\ 4.0 \mathrm{u} & 4.0 \mathrm{u} & \mathrm{NC}\end{array}$

DILUTION

FACTOR (REP)

$== \pm= \pm=====$

1.0

1. 0

1.0

1.0

1.0

1.0

1.0 
Lionville Laboratory, Inc.

INORGANICS LABORATORY CONTROL STANDARDS REPORT 03/28/05

CLIENT: BECHTEL NEVADA V2428

WORK ORDER: 60052-001-001-0001-00

SAMPLE SITE ID

LCS1 05L0152-LC1

LCS 1

$05 C 0056-$ LC1
ANALYTE

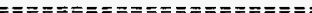

Silver, LCS

Arsenic, LCS

Barium, LCS

Cadmium, LCS

Chromium, LCS

Lead, LCS

Selenium, LCS

Mercury, LCS
LVL LOT \#: 0503L971

$\begin{array}{rrrr}\text { SPIKED } & \text { SPIKED } & & \\ \text { SAMPLE } & \text { AMOUNT } & \text { UNITS } & \text { \&RECOV } \\ ===== & ==\pi== & === \pm= & ===== \\ 497 & 500 & \text { UG } / L & 99.3 \\ 9840 & 10000 & \text { UG } / L & 98.4 \\ 4970 & 5000 & \text { UG } / L & 99.4 \\ 248 & 250 & \text { UG } / L & 99.1 \\ 500 & 500 & \text { UG } / L & 99.9 \\ 2520 & 2500 & \text { UG } / L & 100.8 \\ 10000 & 10000 & \text { UG } / L & 100.3\end{array}$

5.0 UG $/ \mathrm{L}$

103.9 


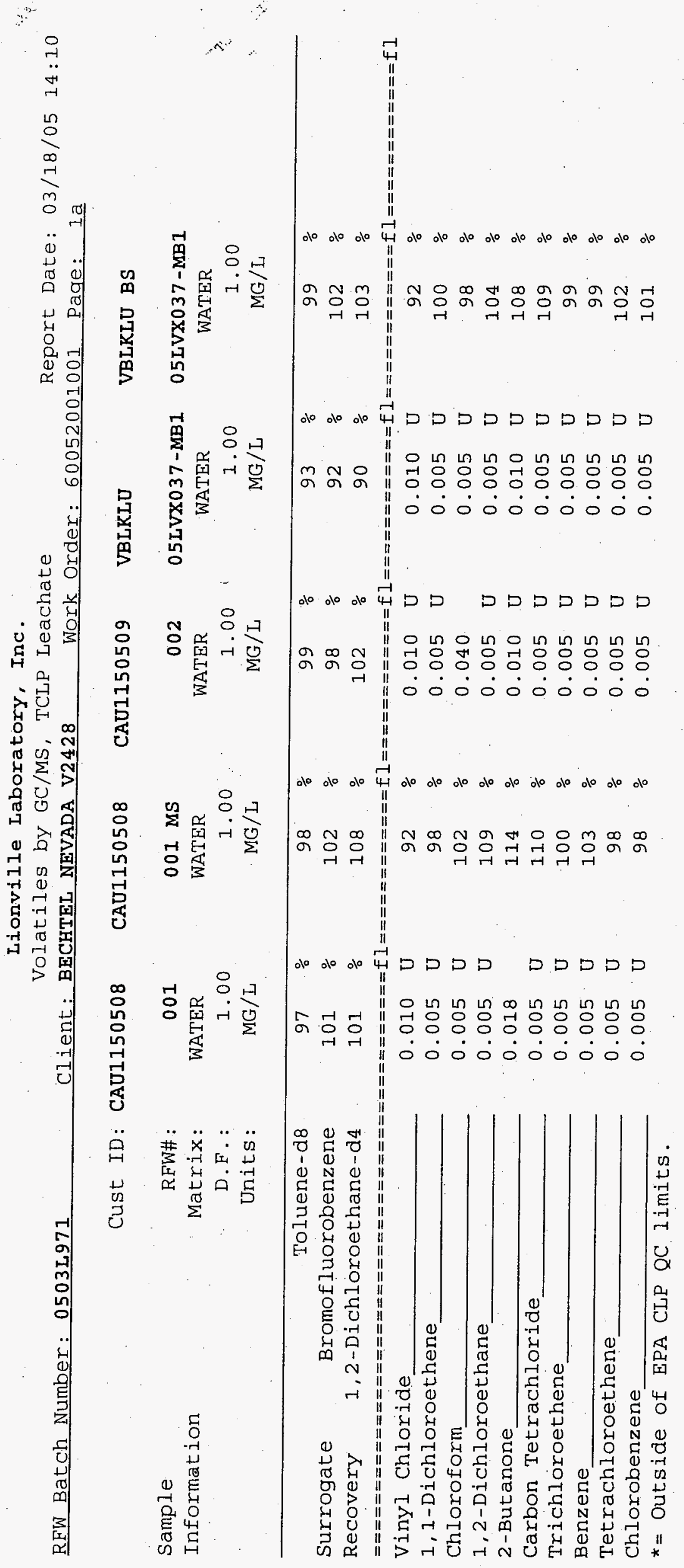


$2 A$

WATER VOLATILE SURROGATE RECOVERY

Lab Name: Lionville Labs, Inc.

Contract: $0052-01-01$

Lab Code: Lionvi Case No.:

SAS NO.:

SDG NO.:

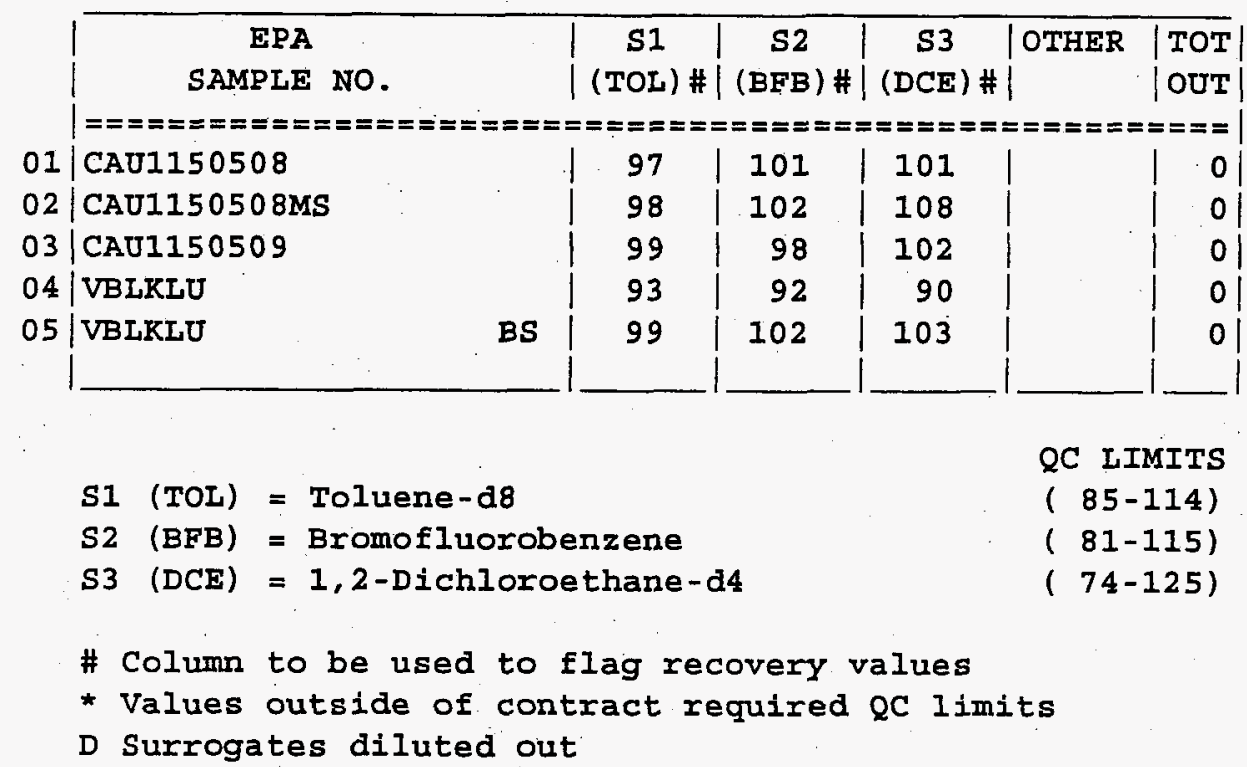


3A

WATER VOLATILE MATRIX SPIKE RECOVERY
Lab Name: Lionville Labs, Inc.
Contract : $\quad$ 0052-01-01
Lab Code: Lionvi Case No.
SAS NO.: —
MATRIX Spike - EPA Sample No.: CAU1150508
Level: (low/med) LOW

\begin{tabular}{|c|c|c|c|c|c|c|}
\hline COMPOUND & $\begin{array}{l}\text { SPIKE } \\
\text { ADDED } \\
(M G / L)\end{array}$ & $\begin{array}{l}\text { SAMPLE } \\
\text { CONCENTRATION } \\
\text { (MG/L) }\end{array}$ & $\begin{array}{c}\text { MS } \\
\text { CONCENTRATION } \\
\text { (MG/L) }\end{array}$ & $\begin{array}{l}\text { MS } \\
\% \\
\text { REC \# }\end{array}$ & $\begin{array}{r}Q \\
\text { LII } \\
1\end{array}$ & $\begin{array}{l}\text { C } \\
\text { MITS } \\
\text { REC }\end{array}$ \\
\hline \multicolumn{7}{|c|}{ 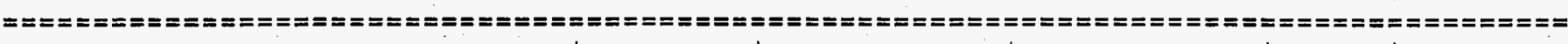 } \\
\hline Vinyl Chloride & 0.0500 & $0 \quad 0 \quad 1$ & $0.0462 \quad \mid$ & 921 & 50 & -150 \\
\hline 1,1-Dichloroethene. & 0.0500 & 0 & 0.0488 & 98 & 61 & -145 \\
\hline Chloroform & 0.0500 & 0 & 0.0510 & 102 & 50 & -150 \\
\hline 1,2-Dichloroethane & 0.0500 & 0 & 0.0544 & 109 & 50 & -150 \\
\hline 2-Butanone & 0.0500 & 0.0185 & 0.0757 & 114 & 50 & -150 \\
\hline Carbon Tetrachloride & 0.0500 & 0 & 0.0549 & 110 & 50 & -150 \\
\hline Trichloroethene. & 0.0500 & 0 & 0.0502 & 100 & 71 & -120 \\
\hline Benzene & 0.0500 & 0 & 0.0513 & 103 & 76 & -127 \\
\hline Tetrachloroe thene_ & 0.0500 & 0 & 0.0491 & 98 & 50 & -150 \\
\hline Chlorobenzene & 0.0500 & 0 & 0.0490 & 98 & 75 & -130 \\
\hline
\end{tabular}

\# Column to be used to flag recovery value with an asterisk

* Values outside of QC limits

Spike Recovery: $\underline{0}$ out of $\underline{10}$ outside limits

COMMENTS : 
Lab Name: Iionville Labs, Inc.

Lab Code: Iionvi Case No.: MATRIX Spike - EPA Sample No.: VBLKLU
Contract: $\quad$ 0052-01-01

SAS No.:

SDG NO.:

Level: (low/med) LOW

\begin{tabular}{|c|c|c|c|c|c|c|}
\hline COMPOUND & $\begin{array}{l}\text { SPIKE } \\
\text { ADDED } \\
\text { (MG/L) }\end{array}$ & \begin{tabular}{|} 
SAMPLE \\
CONCENTRATION \\
(MG $/ L)$
\end{tabular} & $\left\{\begin{array}{c}\text { MS } \\
\text { CONCENTRATION } \\
\text { (MG/L) }\end{array}\right.$ & $\begin{array}{l}\text { MS } \\
\% \\
\text { REC \# }\end{array}$ & & $\begin{array}{l}\text { C } \\
\text { MITS } \\
\text { REC }\end{array}$ \\
\hline \multicolumn{7}{|c|}{ 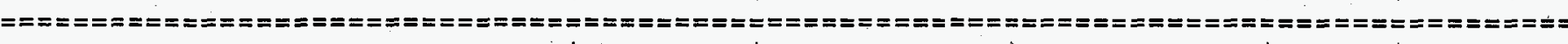 } \\
\hline Vinyl Chloride & 0.0500 & $\begin{array}{lll}1 & 0 & 1\end{array}$ & 0.0462 & 92 & 50 & -150 \\
\hline 1,1-Dichloroethene & 0.0500 & 0 & 0.0499 & 100 & 61 & -145 \\
\hline Chloroform & 0.0500 & 0 & 0.0491 & 98 & 50 & -150 \\
\hline 1, 2-Dichloroe thane & 0.0500 & 0 & 0.0518 & 104 & 50 & -150 \\
\hline 2-Butanone & 0.0500 & 0 & 0.0542 & 108 & 50 & -150 \\
\hline Carbon Tetrachloride & 0.0500 & 0 & 0.0543 & 109 & 50 & -150 \\
\hline Trichloroe thene & 0.0500 & 0 & 0.0493 & 99 & 71 & -120 \\
\hline Benzene & 0.0500 & 0 & 0.0494 & 99 & 76 & -127 \\
\hline Tetrachloroethene & 0.0500 & 0 & 0.0510 & 102 & 50 & -150 \\
\hline Chlorobenzene & 0.0500 & 0 & 0.0505 & 101 & 75 & -130 \\
\hline
\end{tabular}

\# Column to be used to flag recovery value with an asterisk

* Values outside of QC limits

Spike Recovery: 으 out of 10 outside limits

COMMENTS : 
$4 \mathrm{~A}$

VOLATILE METHOD BLANK SUMMARY

Lab Name: Lionville Laboratory Inc. Contract: 60052-001-001-0001-00

Lab Code: LVLI Case No.: SAS No.:

Lab File ID: $\quad$ X031605

Lab Sample ID: 05LVX037-MBI

Date Analyzed: $\quad 03 / 16 / 5$

Time Analyzed: $\quad 1046$

Matrix: (soil/water) WATER

Level: (low/med) LOW

Instrument ID: $\quad 5970 \mathrm{X}$

THIS METHOD BLANK APPLIES TO THE FOLLOWING SAMPLES, MS and MSD:

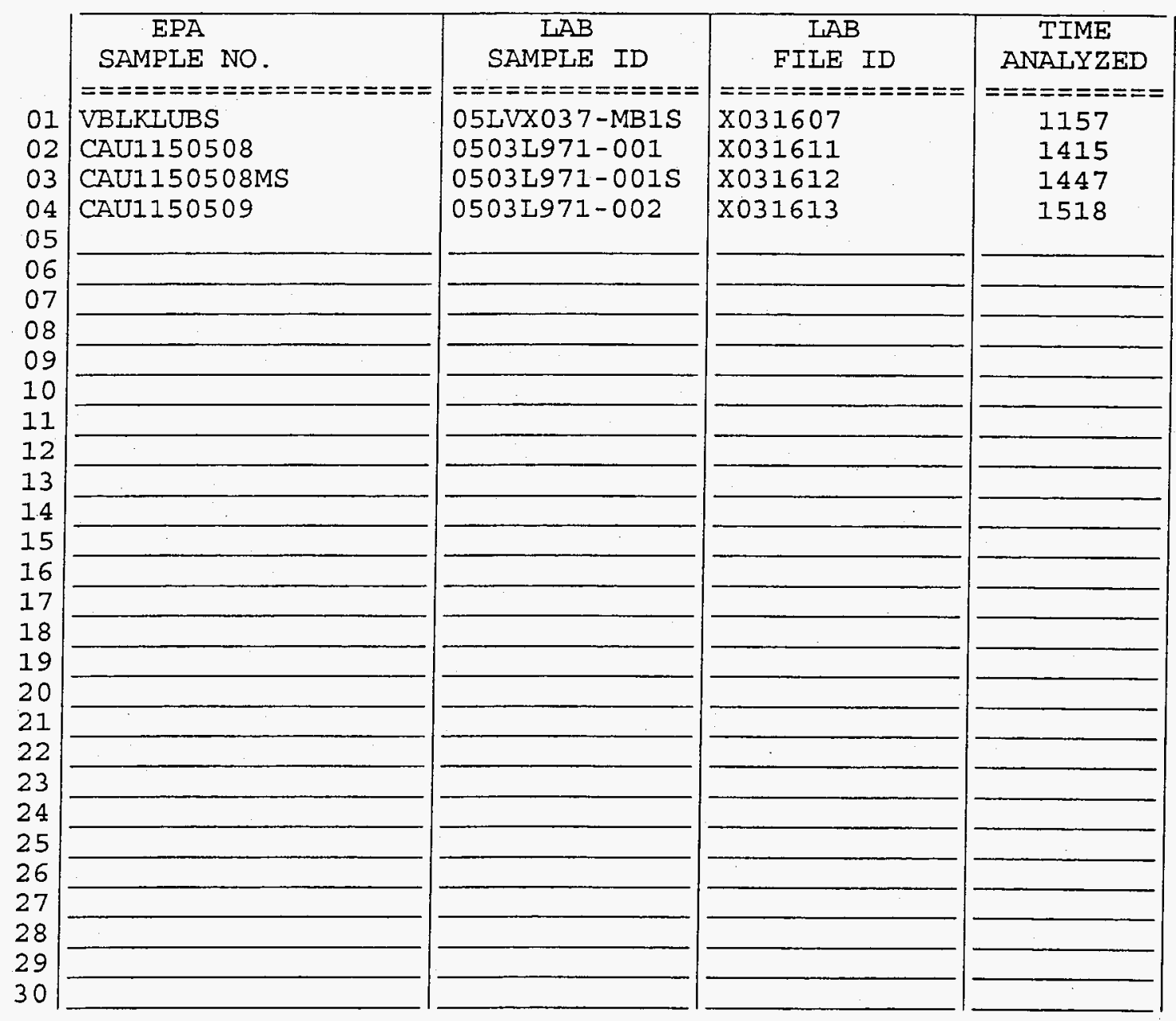

COMMENTS :

page 1 of 1 


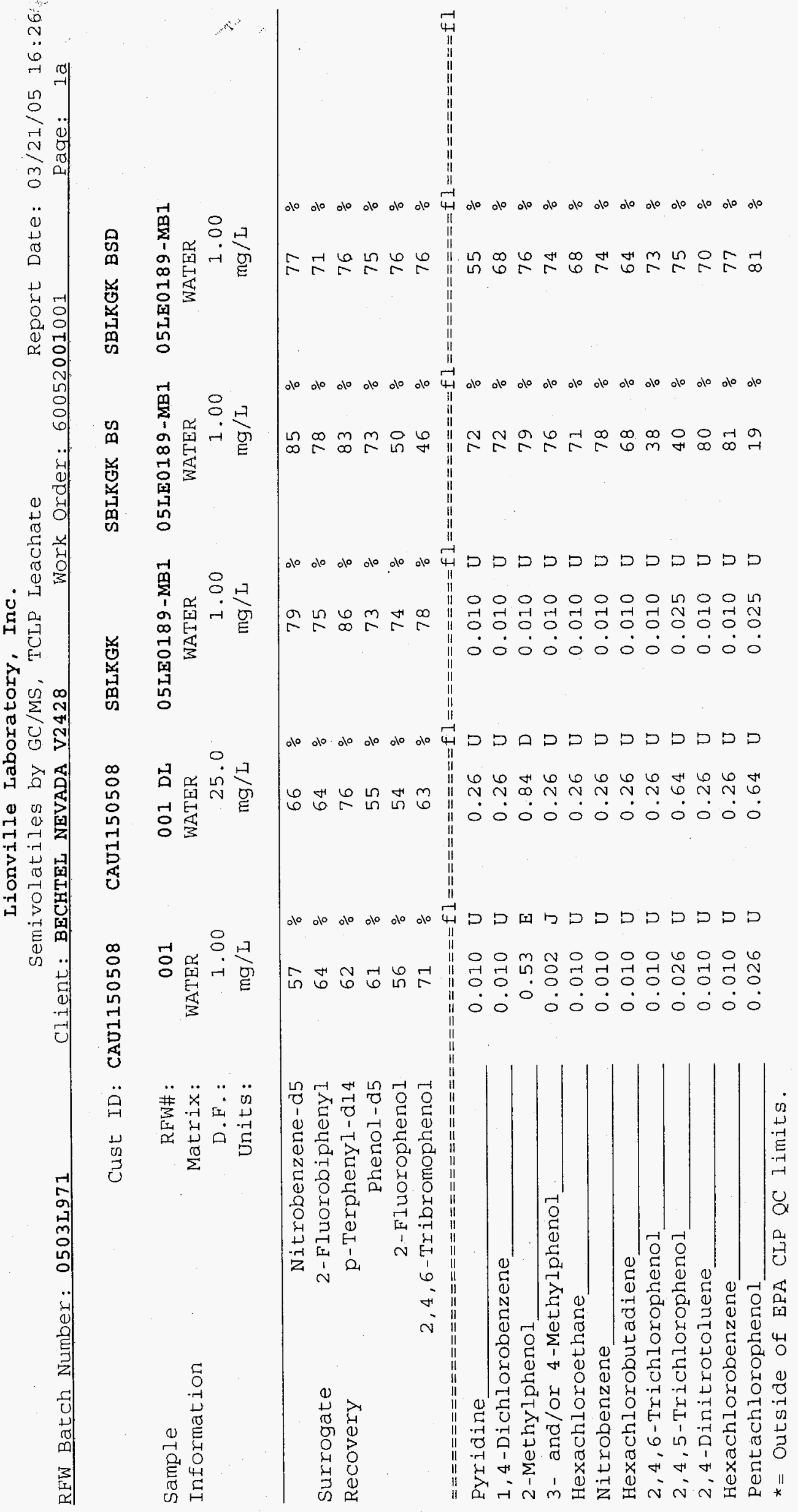


Case No.: BECHTEL NEVADA V2428

RFW Lot No.: 0503L971

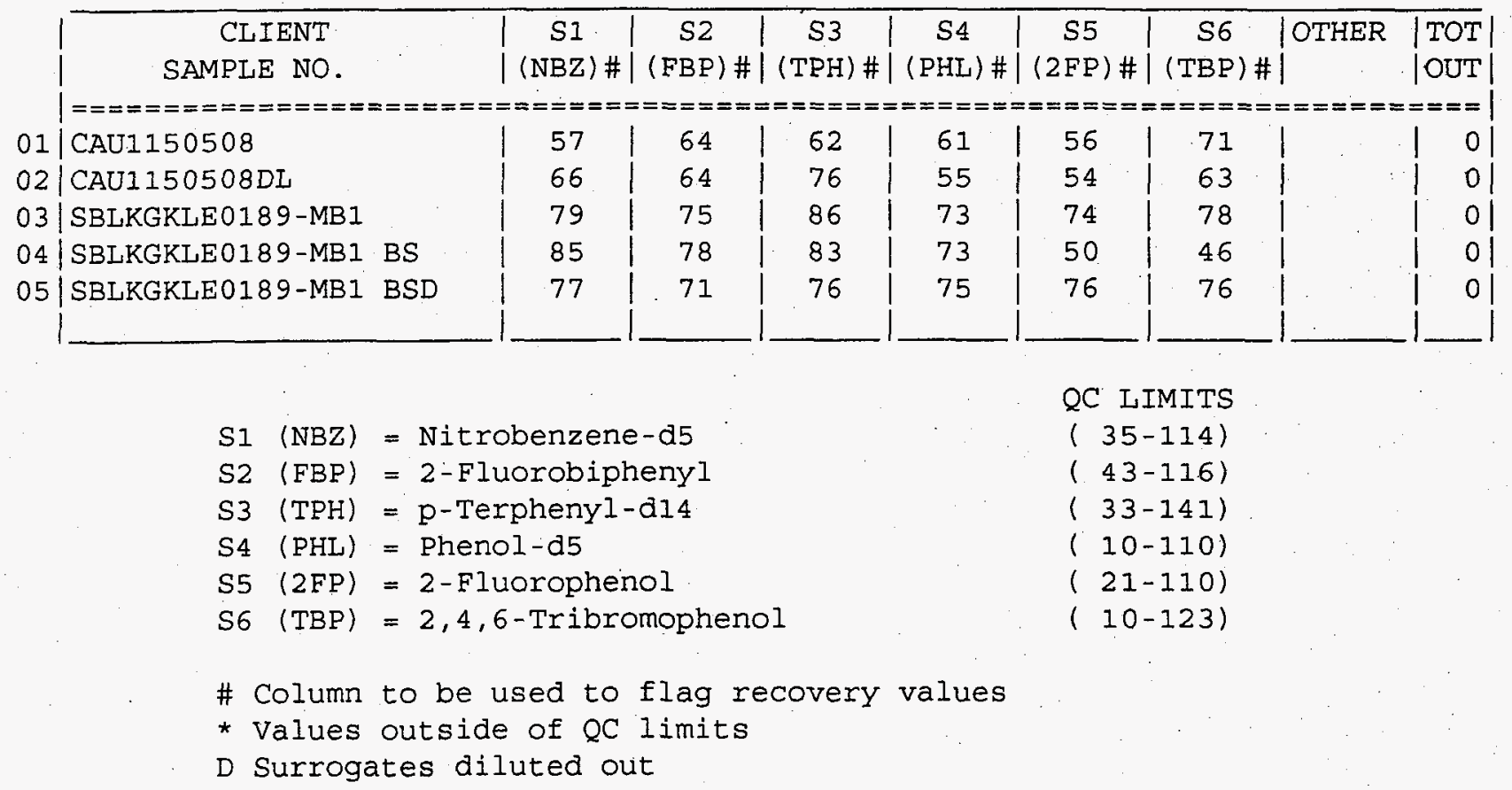


$3 \mathrm{C}$

WATER SEMIVOLATILE BLANK SPIKE/BLANK SPIKE DUPLICATE RECOVERY

Lab Name: Lionville Labs, Inc.

Case No.: BECHTEL NEVADA V2428

BLANK Spike - Sample No.: SBLKGKLE0189-MBI
Contract: $\underline{0052-01-01}$

RFW Lot No.: 0503L971

\begin{tabular}{|c|c|c|c|c|c|c|}
\hline COMPOUND & $\begin{array}{l}\text { SPIKE } \\
\text { ADDED } \\
(\mathrm{mg} / \mathrm{L})\end{array}$ & $\left\{\begin{array}{c}\text { SAMPLE } \\
\text { CONCENTRATION } \\
(\mathrm{mg} / \mathrm{L})\end{array}\right.$ & $\mid \begin{array}{c}\text { BS } \\
\text { CONCENTRATION } \\
(\mathrm{mg} / \mathrm{L})\end{array}$ & $\begin{array}{l}\text { BS } \\
\% \\
\text { REC \# }\end{array}$ & \multicolumn{2}{|c|}{$\begin{array}{l}Q C \\
\text { LIMITS }\end{array}$} \\
\hline 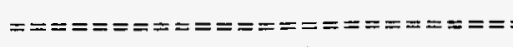 & & & & & & \\
\hline Pyridine & 0.0500 & 0 & 0.0359 & 72 & 9 & -141 \\
\hline 1,4-Dichlorobenzene_ & 0.0500 & 0 & 0.0358 & 72 & 36 & -97 \\
\hline 2-Methylphenol & 0.0500 & 0 & 0.0397 & 79 & 9 & -141 \\
\hline 3- and/or 4-Methylphenol. & 0.100 & 0 & 0.0759 & 76 & 9 & -141 \\
\hline Hexachloroethane & 0.0500 & 0 & 0.0354 & 71 & 9 & -141 \\
\hline Nitrobenzene & 0.0500 & 0 & 0.0390 & 78 & 9 & -141 \\
\hline Hexachlorobutadiene & 0.0500 & 0 & 0.0340 & 68 & 9 & -141 \\
\hline 2,4,6-Trichlorophenol. & 0.0500 & 0 & 0.0189 & 38 & 9 & -141 \\
\hline $2,4,5$-Trichlorophenol_ & 0.0500 & 0 & 0.0201 & 40 & 9 & -141 \\
\hline 2.4-Dinitrotoluene & 0.0500 & 0 & 0.0401 & 80 & 24 & -96 \\
\hline Hexachlorobenzene & 0.0500 & 0 & 0.0404 & 81 & 9 & -141 \\
\hline Pentachlorophenol & 0.0500 & 0 & 0.00944 & 19 & 9 & -103 \\
\hline
\end{tabular}

\begin{tabular}{|c|c|c|c|c|c|c|c|c|}
\hline COMPOUND & $\begin{array}{l}\text { SPIKE } \\
\text { ADDED } \\
(\mathrm{mg} / \mathrm{L})\end{array}$ & $\mid \begin{array}{c}\text { BSD } \\
\text { CONCENTRATION } \\
(\mathrm{mg} / \mathrm{L})\end{array}$ & $\begin{array}{l}\text { BSD } \\
\frac{\circ}{6} \\
\text { REC \# }\end{array}$ & $\begin{array}{c}\frac{\circ}{0} \\
R P D\end{array}$ & $\#$ & \multicolumn{3}{|c|}{ QC LIMITS } \\
\hline \multicolumn{9}{|l|}{$====== \pm===$} \\
\hline Pyridine & 0.0500 & 0.0275 & 55 & 26 & & 50 & 9 & -141 \\
\hline 1,4-Dichlorobenzene & 0.0500 & 0.0342 & 68 & 5 & & 28 & 36 & -97 \\
\hline 2-Methylphenol & 0.0500 & 0.0380 & 76 & 3 & & 50 & 9 & -141 \\
\hline 3- and/or 4-Methylphenol. & 0.100 & 0.0738 & 74 & 2 & & 50 & 9 & -141 \\
\hline Hexachloroethane & 0.0500 & 0.0340 & 68 & 4 & & 50 & 9 & -141 \\
\hline Nitrobenzene & 0.0500 & 0.0370 & 74 & 5 & & 50 & 9 & -141 \\
\hline Hexachlorobutadiene & 0.0500 & 0.0320 & 64 & 6 & & 50 & 9 & -141 \\
\hline $2,4,6$-Trichlorophenol_ & 0.0500 & 0.0367 & 73 & 63 & * & 50 & 9 & -141 \\
\hline $2,4,5$-Trichlorophenol & 0.0500 & 0.0374 & 75 & 61 & * & 50 & 9 & -141 \\
\hline 2,4-Dinitrotoluene_ & 0.0500 & 0.0352 & 70 & 13 & & 38 & 24 & -96 \\
\hline Hexachlorobenzene_ & 0.0500 & 0.0384 & 77 & 5 & & 50 & 9 & -141 \\
\hline Pentachlorophenol. & 0.0500 & 0.0406 & 81 & 123 & * & 50 & 9 & -103 \\
\hline
\end{tabular}

\# Column to be used to flag recovery and RPD values with an asterisk

* Values outside of QC limits

RPD: $\quad 3$ out of 12 outside limits

Spike Recovery: $\underline{0}$ out of $\underline{24}$ outside limits

COMMENTS : 


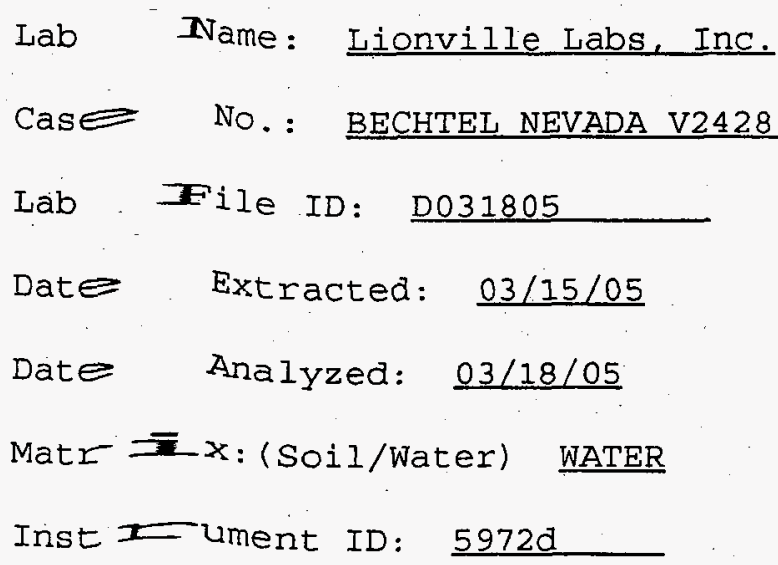

THIS METHOD BLANK APPLIES TO THE FOLLOWING SAMPLES, MS AND MSD:

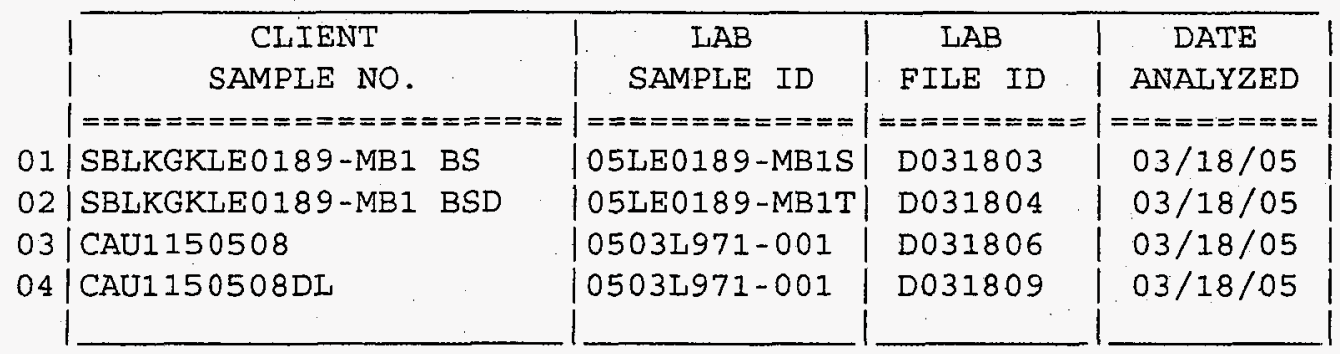

COMMEITIS : 
Lionville Laboratory, Inc.

INORGANICS DATA SUMMARY REPORT 03/15/05

CLIENT: BECHTEL NEVADA V2428

WORK ORDER: 60052-001-001-0001-00

SAMPLE

$=== \pm==$

$-001$

SITE ID

CAU1150508
ANALYTE

$\mathrm{pH}$
LVL LOT \# : 0503L97I

\begin{tabular}{|c|c|c|}
\hline & & REPORTING \\
\hline RESULT & UNITS & LIMIT \\
\hline$===\leq=-=$ = & $====\pi=$ & $==x=m=$ \\
\hline
\end{tabular}

DILUTION

FACTOR

$=x=== \pm==$

1.0 

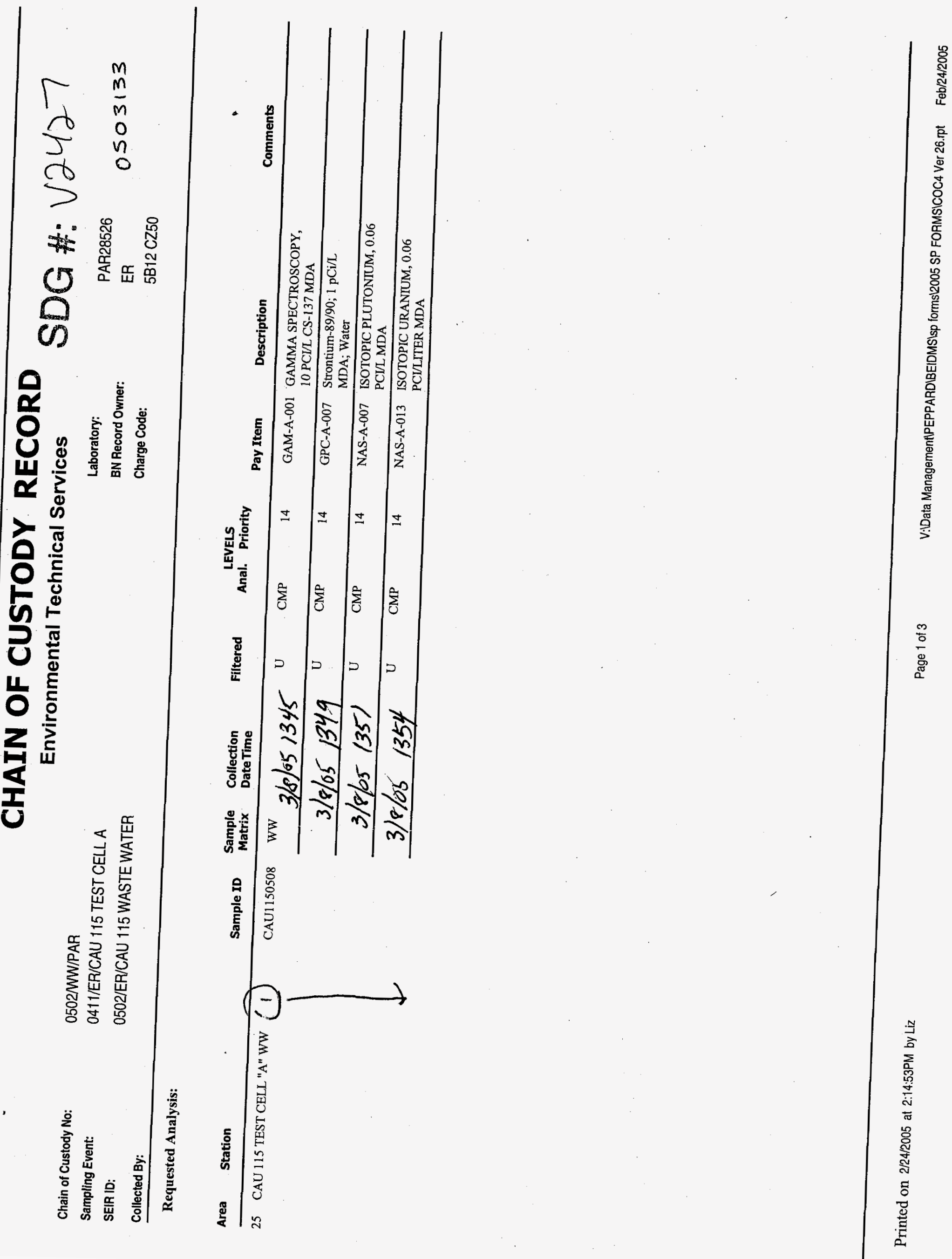


\title{
Gamma Spectroscopy Results
}

\author{
PAI 713 Rev 8 \\ Sample Results
}

Lab Name: Paragon Analytics

Work Order Number: 0503133

Client Name: Bechtel Nevada

ClientProject ID: 0411/ ER / CAU 115 Test Cell A V2427

\begin{tabular}{|c|}
\hline $\begin{array}{l}\text { fleld IDF CAU1150508 } \\
\text { LablD. } \\
0503133-1\end{array}$ \\
\hline
\end{tabular}

Library: LNG_GAM_A_00
Sample Matrix: WATER

Prep SOP: PAI 739 Rev 8

Date Collected: 08-Mar-05

Date Prepared: 16-Mar-05

Date Analyzed: 16-Mar-05
Prep Batch: GS050316-10

QCBatchID: GS050316-10-1

Run ID: gs050316-10a

Count Time: 400 minutes

Report Basis: Unfiltered
Final Aliquot: $1000 \mathrm{ml}$

Prep Basis: Unfiltered

Moisture(\%): NA

Result Units: $\mathrm{pCi} / \mathrm{l}$

File Name: 050417D10A

\begin{tabular}{|c|c|c|c|c|}
\hline CASNO & Target Nuclide & Result +/- 2 s TPU & MDC & Lab Qualifier \\
\hline $14331-83-0$ & Ac-228 & $3.22 E+00+/-1.14 E+01$ & $1.90 E+01$ & $U$ \\
\hline $14596-10-2$ & $A m-241$ & $1.92 E-01+/-1.21 E+01$ & $2.01 E+01$ & U \\
\hline $14762-78-8$ & $\mathrm{Ce}-144$ & $7.25 E+\infty 0+/-1.15 E+01$ & $1.90 E+01$ & $U$ \\
\hline $10198-40-0$ & Co-60 & $6.02 E-01+/-2.12 E+00$ & $3.57 E+00$ & U \\
\hline $13967-70-9$ & Cs-134 & $-1.37 E+00+/-3.48 E+00$ & $5.86 E+00$ & $U$ \\
\hline $10045-97-3$ & Cs-137 & $-4.82 E-01+/-2.09 E+00$ & $3.56 \mathrm{E}+00$ & $U$ \\
\hline $14683-23-9$ & Eu-152 & $2.88 E+00+/-1.33 E+01$ & $2.23 E+01$ & $U$ \\
\hline $15585-10-1$ & Eu-154 & $1.18 \mathrm{E}+00+/-1.09 \mathrm{E}+01$ & $1.84 \mathrm{E}+01$ & $U$ \\
\hline $14391-16-3$ & Eu-155 & $6.55 \mathrm{E}-01+/-6.50 \mathrm{E}+00$ & $1.09 \mathrm{E}+01$ & U \\
\hline $13966-00-2$ & $K-40$ & $1.21 E+02+1-4.53 E+01$ & $6.77 E+01$ & \\
\hline $15092-94-1$ & $\mathrm{~Pb}-212$ & $3.92 E+00+/-4.76 E+00$ & $7.79 \mathrm{E}+00$ & $U$ \\
\hline $14834-73-2$ & Pm-144 & $-2.09 \mathrm{E}+00+/-4.55 \mathrm{E}+00$ & $7.60 \mathrm{E}+00$ & $U$ \\
\hline $14834-74-3$ & $P m-146$ & $-7.87 E-01+/-2.15 E+00$ & $3.67 \mathrm{E}+00$ & $U$ \\
\hline $13967-48-1$ & Ru-106 & $-7.04 \mathrm{E}-01+/-1.96 \mathrm{E}+01$ & $3.30 E+01$ & $U$ \\
\hline $14683-10-4$ & Sb-124 & $7.08 E+00+/-2.42 E+00$ & $3.50 E+00$ & $\mathrm{TI}$ \\
\hline
\end{tabular}

\section{Comments:}

\footnotetext{
Qualifiers/Flags:

$U$ - Result is less than the sample specific MDC or less than the associated TPU

Y1 - Chemical Yield is in control at 100-110\%. Quantitative Yield is assumed.

Y2 - Chemical Yield outside default limits.

$L T$ - Result is less than Requested MDC, greater than sample specific MDC.

M3 - The requested MDC was not met, but the reported

activity is greater than the reported MDC.

$M$ - The requested MDC was not met.
}

Abbreviations:

TPU - Total Propagated Uncertainty (see PAI SOP 743)

MDC - Minimum Detectable Concentration (see PAI SOP 709)

$\mathrm{BDL}$ - Below Defection Limit

Data Package ID: GSW0503133-1 


\title{
Gamma Spectroscopy Results
}

\author{
PAI 713 Rev 8
}

Sample Results

Lab Name: Paragon Analytics

Work Order Number: 0503133

Client Name: Bechtel Nevada

ClientProject ID: 0411/ ER / CAU 115 Test Cell A V2427

\begin{tabular}{|l|l|l|}
\hline Tield ID. & CAU1150508 \\
Lab. ID & $0503133-1$ \\
\hline
\end{tabular}

Library: LNG_GAM_A_00
Sample Matrix: WATER

Prep SOP: PAI 739 Rev 8

Date Collected: 08-Mar-05

Date Prepared: 16-Mar-05

Date Analyzed: 16-Mar-05
Prep Batch: GS050316-10

QCBatchID: GS050316-10-1

Run ID: gs050316-10a

Count Time: 400 minutes

Report Basis: Unfiltered
Final Aliquot: $1000 \mathrm{ml}$

Prep Basis: Unfiltered

Moisture(\%): NA

Result Units: $\mathrm{pCi} / /$

File Name: 050417D10A

\begin{tabular}{|c|c|c|c|c|}
\hline CASNO & Target Nuclide & Result +/- 2 s TPU & MDC & Lab Qualifier \\
\hline $14234-35-6$ & Sb-125 & $8.46 E-01+/-4.28 E+00$ & $7.80 E+00$ & $U$ \\
\hline $15065-10-8$ & Th-234 & $-1.97 E+01+/-4.86 E+01$ & $8.52 E+01$ & $U$ \\
\hline $15117-96-1$ & U-235 & $1.00 E+01+/-1.15 E+01$ & $1.88 E+01$ & $U$ \\
\hline $13982-36-0$ & Y-88 & $3.88 E+00+/-2.29 E+00$ & $3.57 E+00$ & TI \\
\hline
\end{tabular}

\section{Comments:}

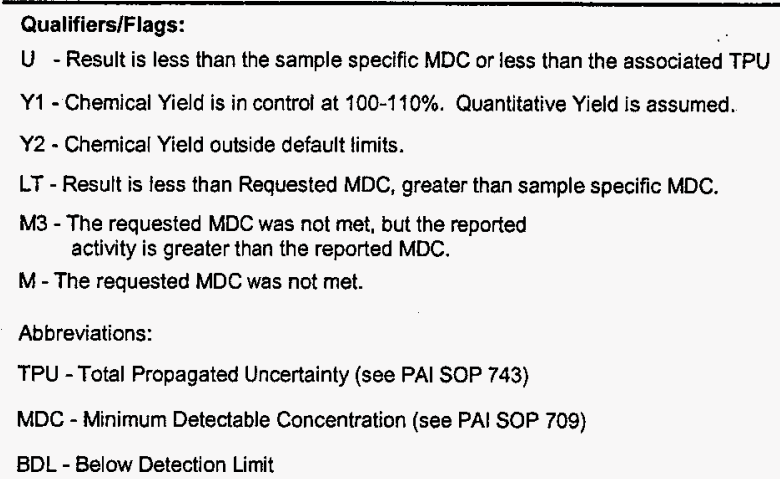

$U$ - Result is less than the sample specific MDC or less than the associated TPU

Y1 - Chemical Yield is in control at 100-110\%. Quantitative Yield is assumed.

Y2 - Chemical Yield outside default limits.

LT - Result is less than Requested MDC, greater than sample specific MDC.

M3 - The requested MDC was not met, but the reported activity is greater than the reported MDC.

$M$ - The requested MDC was not met.

Abbreviations:

TPU - Total Propagated Uncertainty (see PAI SOP 743)

MDC - Minimum Detectable Concentration (see PAI SOP 709)

BDL - Below Detection Limit

Data Package ID: GSW0503133-1

Date Printed: Monday, March 21, 2005

TI - Nuclide identification is tentative.

$R$ - Nuclide has exceeded 8 halfives.

G - Sample density differs by more than $15 \%$ of LCS density. 

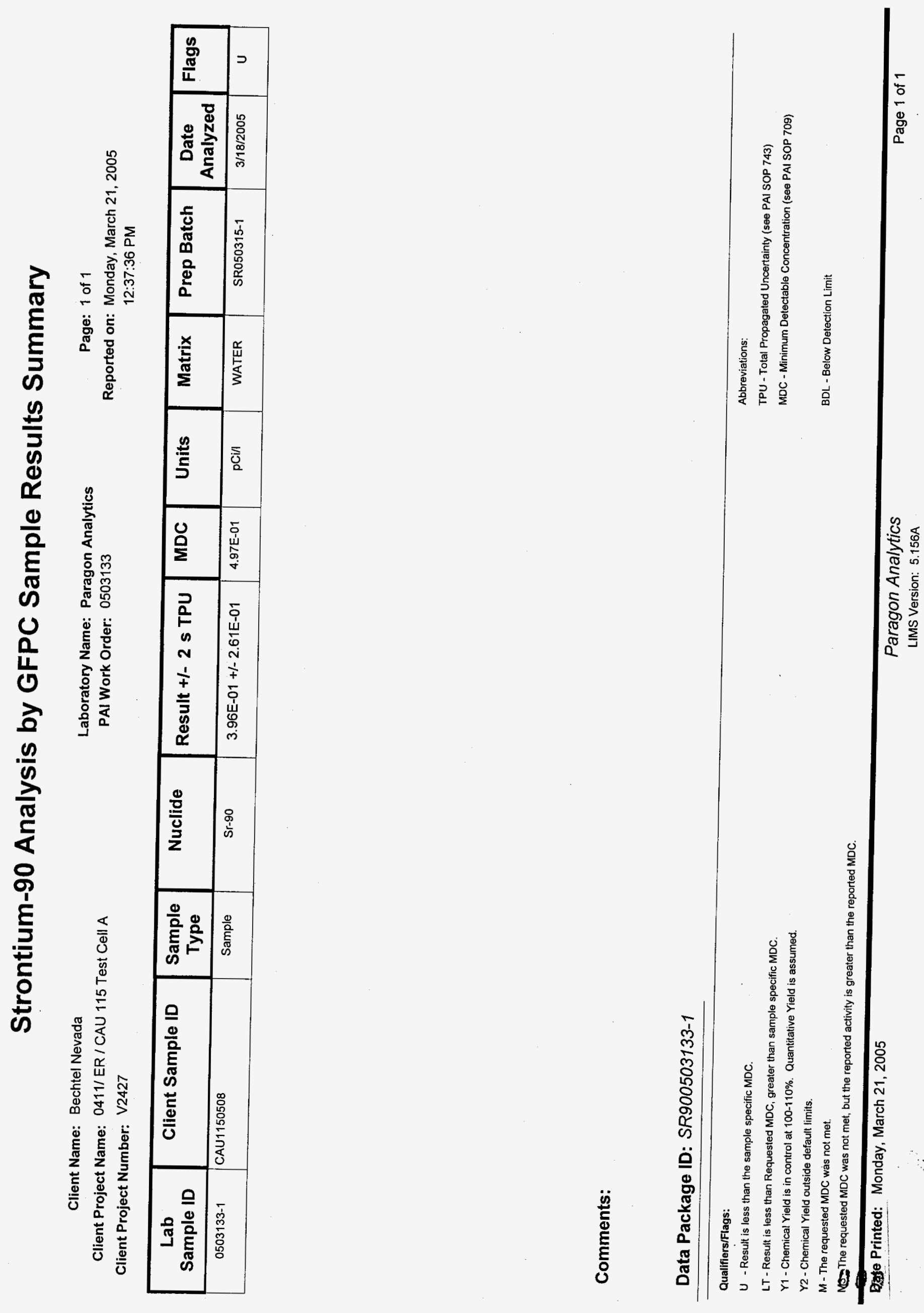

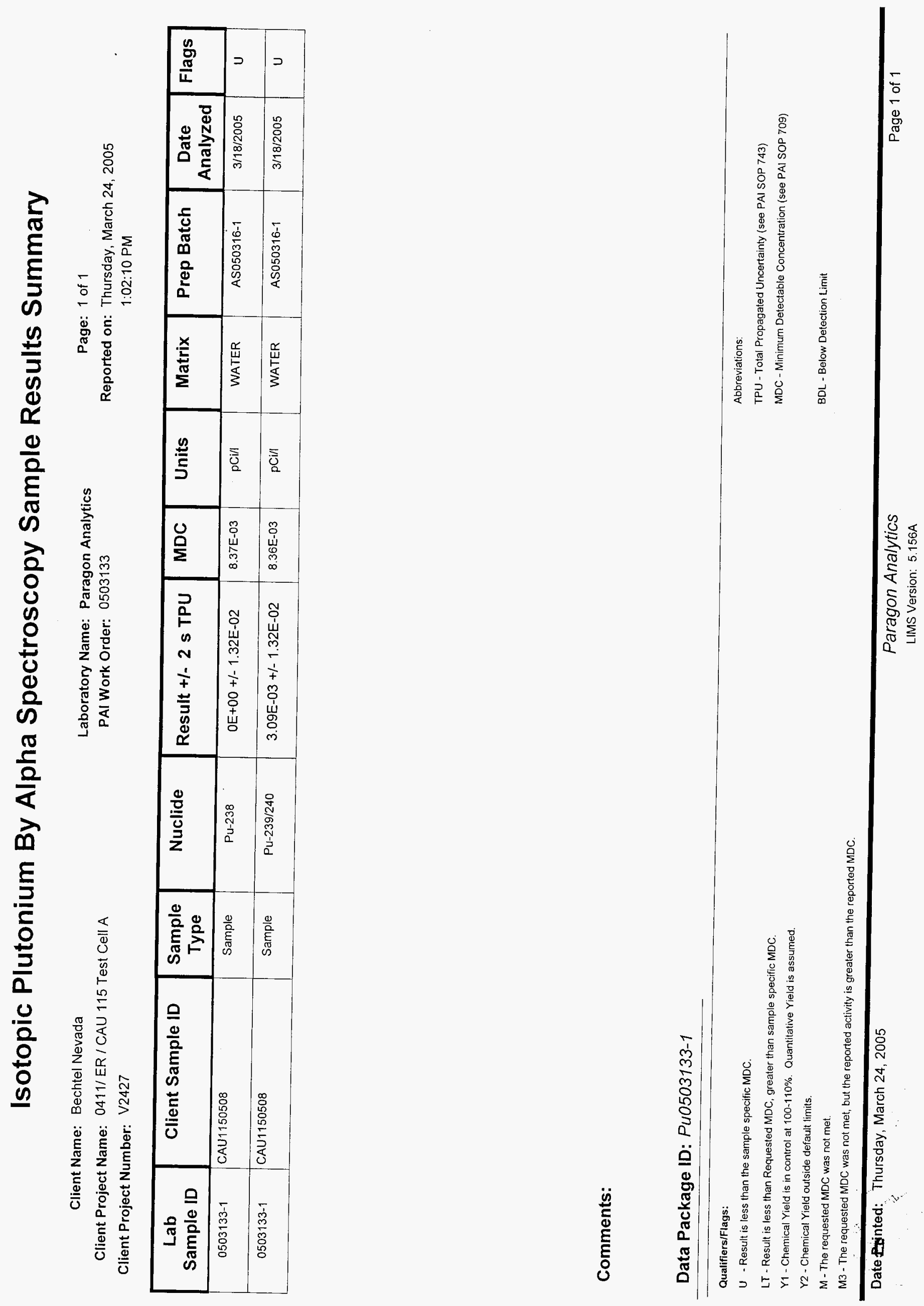

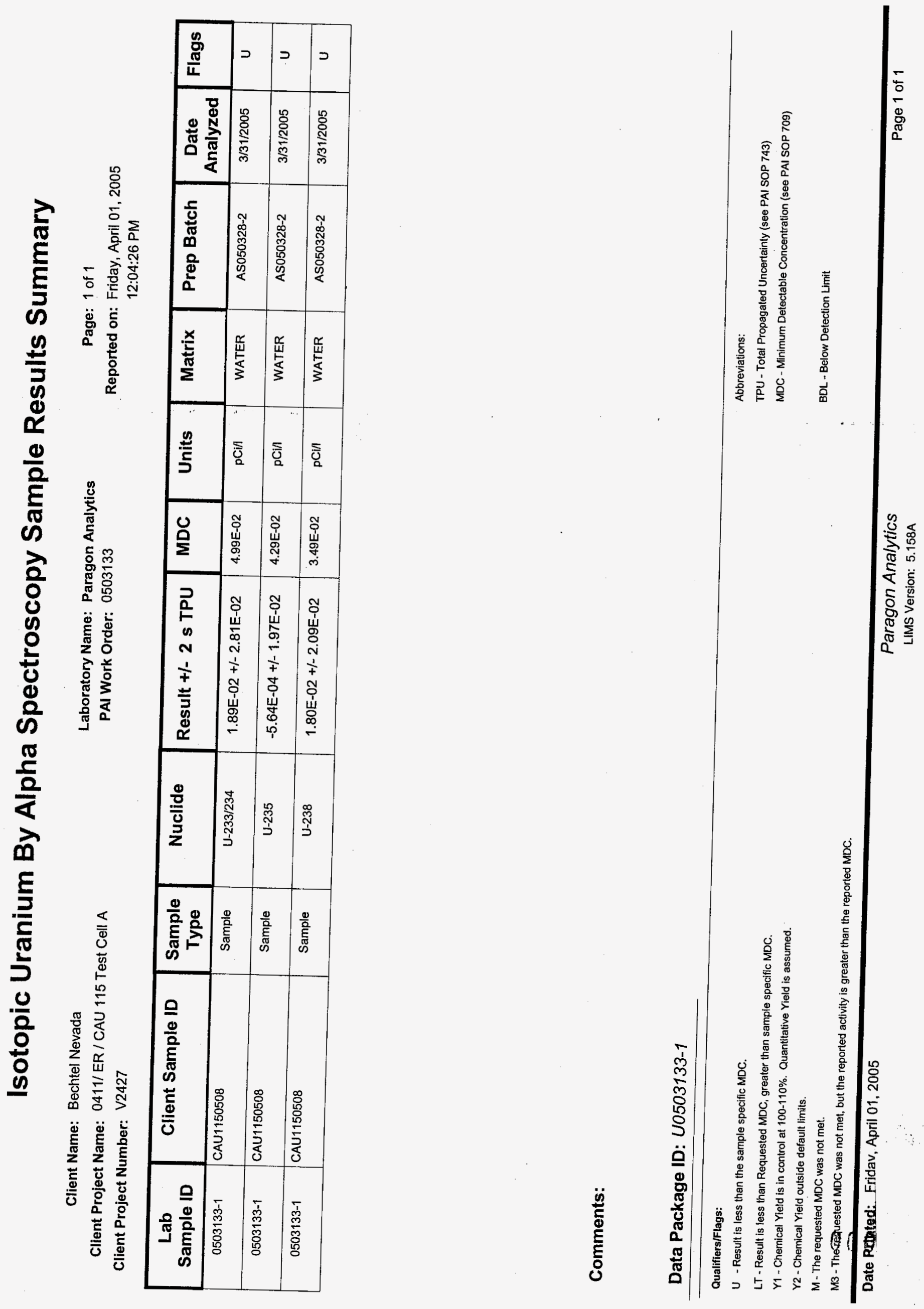


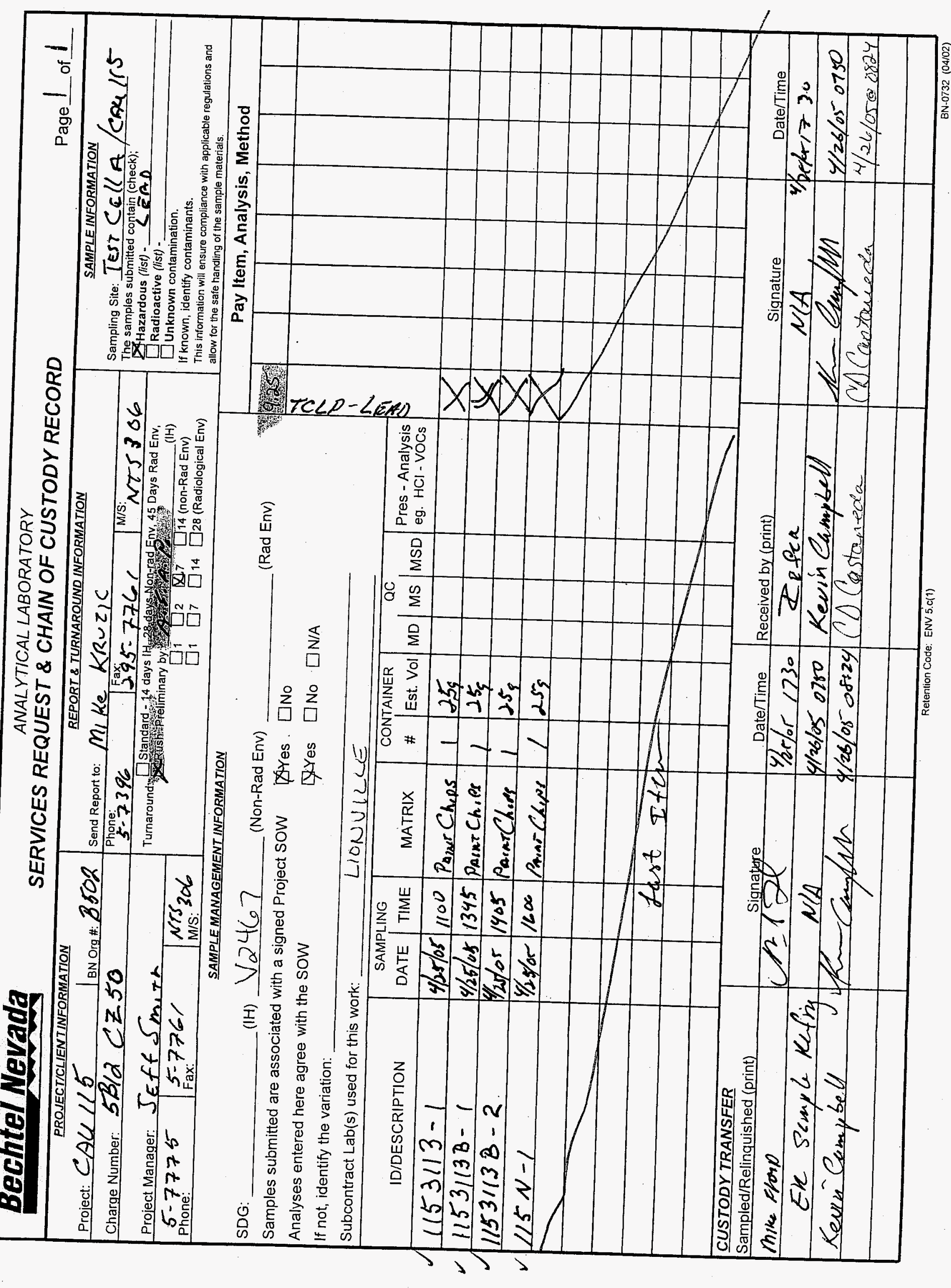


Lionville Laboratory, Inc.

INORGANICS DATA SUMMARY REPORT 05/04/05

CLIENT: BECHTEL NEVADA V2467

WORK ORDER: 60052-001-001-0001-00

\begin{tabular}{|c|c|c|}
\hline SAMPLE & SITE ID & ANALYTE \\
\hline$=\mp====$ & 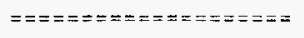 & 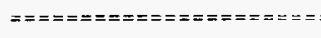 \\
\hline-005 & $1153113-1$ & Lead, TCLP Leachate \\
\hline .006 & $1153113 \mathrm{~B}-1$ & Lead, TCLP Leachate \\
\hline-007 & $1153113 \mathrm{~B}-2$ & Lead, TCLP Leachate \\
\hline 008 & $115 \mathrm{~N}-1$ & Lead, TCLP Leachate \\
\hline
\end{tabular}

LVI LOT \#; $0504 \mathrm{~L} 329$

\begin{tabular}{|c|c|c|c|}
\hline & & REPORTING & DILUTION \\
\hline RESULT & UNLTS & LIMIT & FACTOR \\
\hline$==== \pm==0$ & $==\simeq= \pm=$ & $=== \pm= \pm===$ & $=======$ \\
\hline $21.4 \quad \mathrm{u}$ & UG / L & 11.4 & 6.0 \\
\hline 64.3 & $U G / L$ & 11.4 & 6.0 \\
\hline 106 & UG /L & 11.4 & 6.0 \\
\hline 42.8 & $\mathrm{UG} / \mathrm{L}$ & 11.4 & 6.0 \\
\hline
\end{tabular}


Lionvilie Laboratory, Inc.

INORGANICS METHOD ELANK DATA SUMMARY PAGE 05/04/05

CLIENT: BECHTEL NEVADA V 2467

WORK ORDER: 60052-001-001-0001-00

\begin{tabular}{|c|c|c|}
\hline SAMPLE & SITE ID & ANALYTB \\
\hline$==0===0$ & 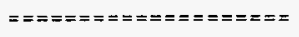 & 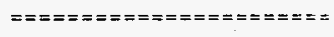 \\
\hline BLANKI & $0.5 \mathrm{~L} 0231-\mathrm{MBI}$ & Lead, TCLP Leachate \\
\hline BLANIK2 & $05 L 0231-1$ BB2 & Lead, TCLP Leachate \\
\hline BLANIK3 & $05 L 0231-M B 3$ & Lead, TCLP Leachate \\
\hline & $05 L 0231-M B 4$ & Lead, TCLP Leachate \\
\hline
\end{tabular}

LVL LOT \#: 0504L329

\begin{tabular}{|c|c|c|c|}
\hline & & REPORTING & DILUTION \\
\hline RESULT & UNITS & LIMIT & FACTOR \\
\hline$======-=$ & $==== \pm=$ & $========$ & $=x===-==$ \\
\hline 1.9 & $\mathrm{UG} / \mathrm{L}$ & 1.9 & 1.0 \\
\hline 11.4 & $\mathrm{UG} / \mathrm{L}$ & 11.4 & 6.0 \\
\hline 11.4 & UG $/ L$ & 11.4 & 6.0 \\
\hline 11.4 & $\mathrm{UG} / \mathrm{L}$ & 11.4 & 6.0 \\
\hline
\end{tabular}


Lionville Laboratory, Ine.

INORGANICS ACCURACY RERORT 05/04/05

CLIENT: BECHTEL NEVADA V2467

WORK ORDER: 60052-001-001-0001-00
LVL LOT \#: 0504L329

$\begin{array}{llccc}\text { SPIKED } & \text { INITIAL } & \text { SPIKED } & & \text { DILUTION } \\ \text { SAMPLE } & \text { RESULT } & \text { AMOUNT } & \% \text { RECOV } & \text { FACTOR (SPK) } \\ ======= & ======= & ====== & ====== & ========= \\ 4380 & 11.4 \mathrm{~L} & 5000 & 87.5 & 6.0\end{array}$


Lionville Laboratory, Inc.

INORGANICS LABORAIORY CONTROL STANDARDS REPORT 05/04/05

CLIENT: BECHTEL NEVADA V2467

WORK ORDER: 60052-001-001-0001-00
LVL LOT \#: $0504 \mathrm{~L} 329$

$\begin{array}{cccc}\text { SPIIKE } & \text { SPIKED } & & \\ \text { SAMPLE } & \text { AMOUNT } & \text { UNITS } & \text { \$QRECOV } \\ ===== & ===== \pm & ===\pi== & ====== \\ 2470 & 2500 & \text { UG } / \mathrm{L} & 98.9\end{array}$

2470

2500

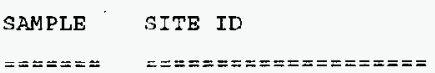

LCS 1
$05 \mathrm{~L} 0231$ - LC1

ANALY"TE

Lead, LCS 
Date: March 2006

THIS PAGE INTENTIONALLY LEFT BLANK 


\section{APPENDIX C}

FINAL RADIOLOGICAL SURVEY RESULTS 
Closure Report - CAU 115

Section: Appendix C

Revision: 0

Date: March 2006

\section{THIS PAGE INTENTIONALLY LEFT BLANK}




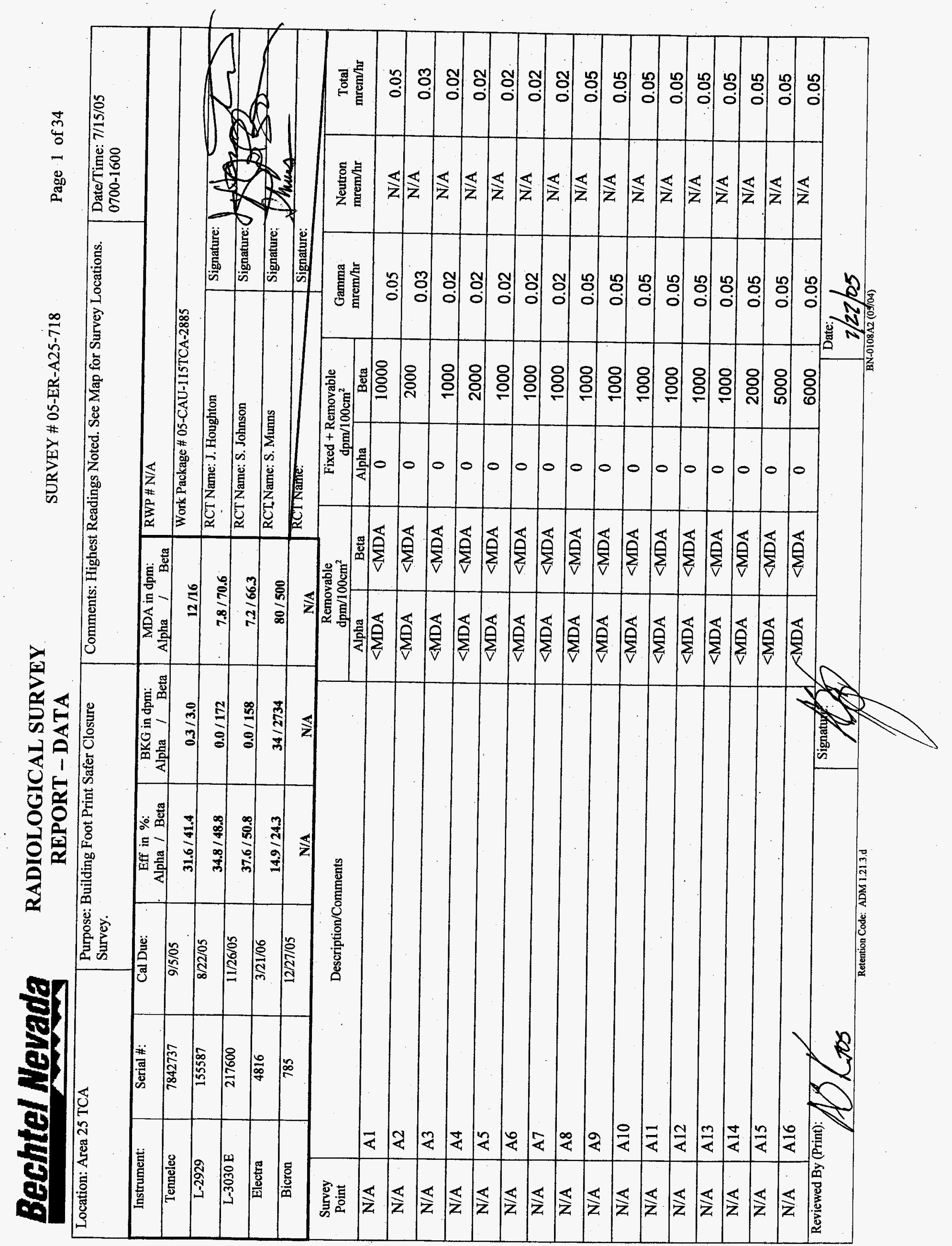




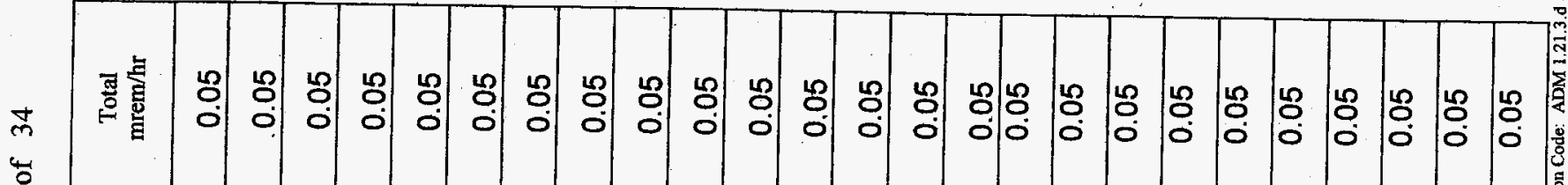

\#

蛋

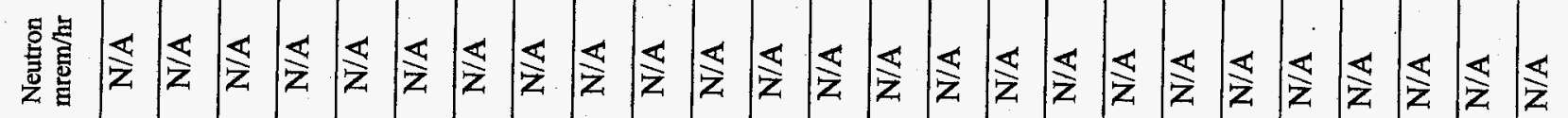

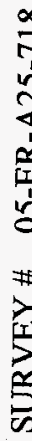

署鲒

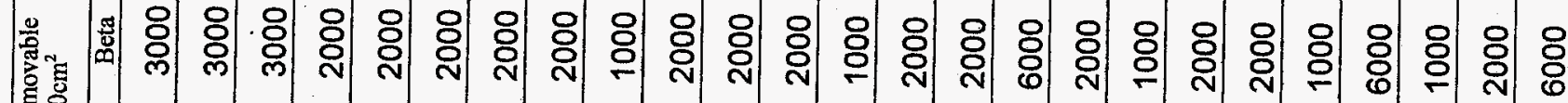
\%

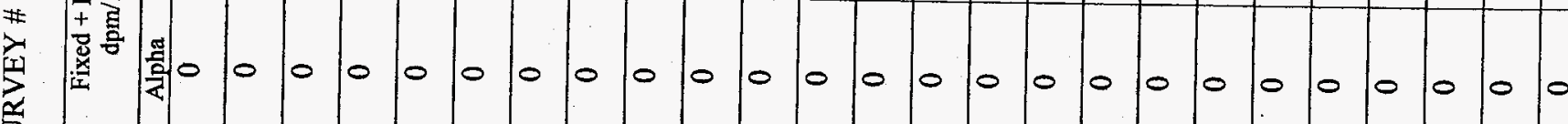

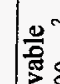

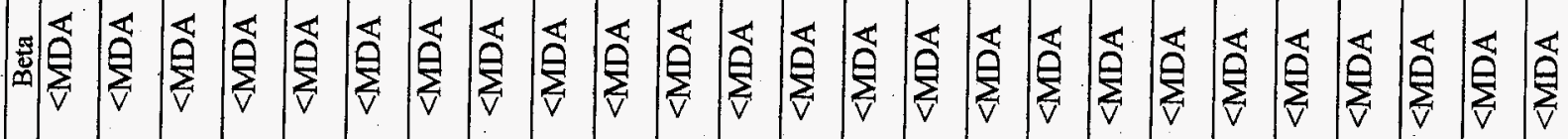

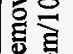

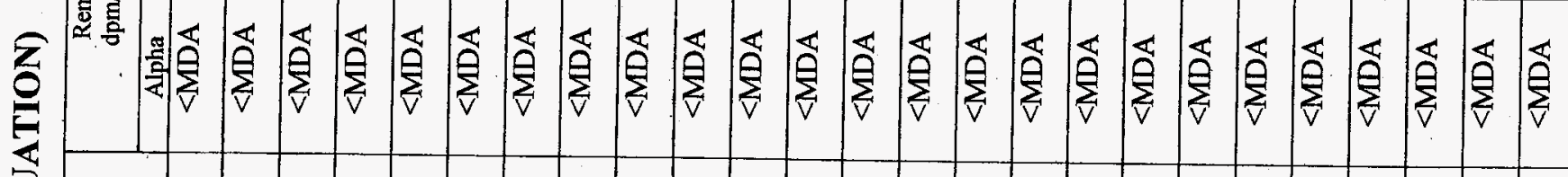
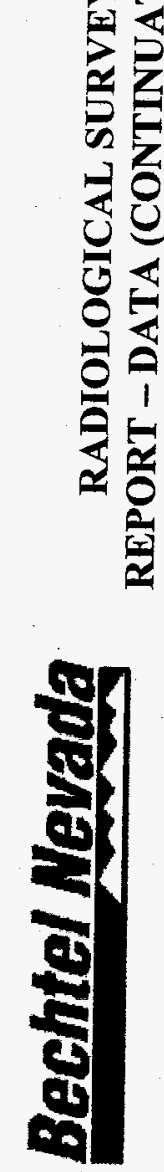

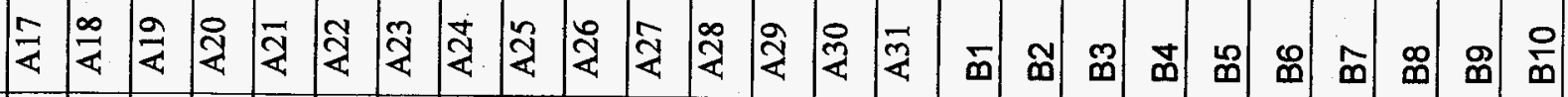

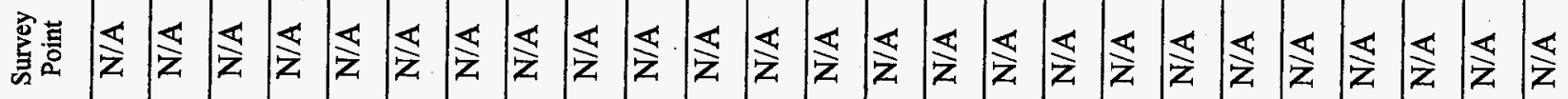


共

4

m

$\#$
0
0
0
0

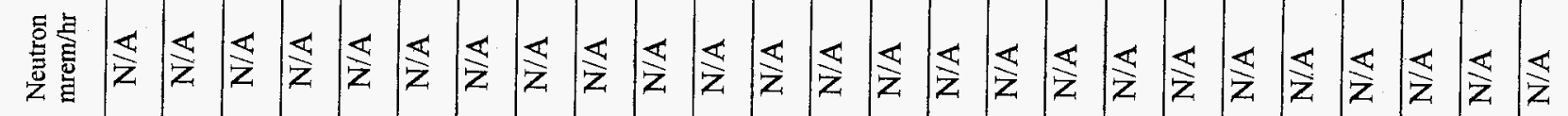

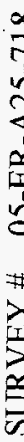

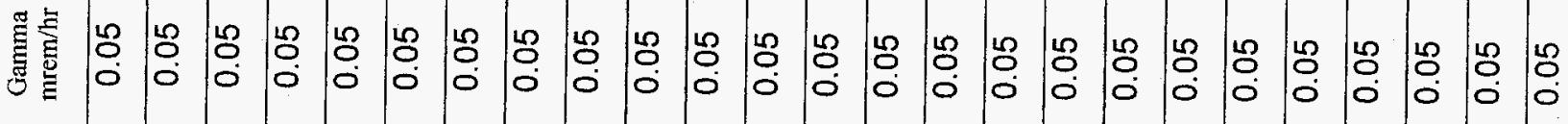

范 乐

\# $+\frac{0}{0}$

至

농

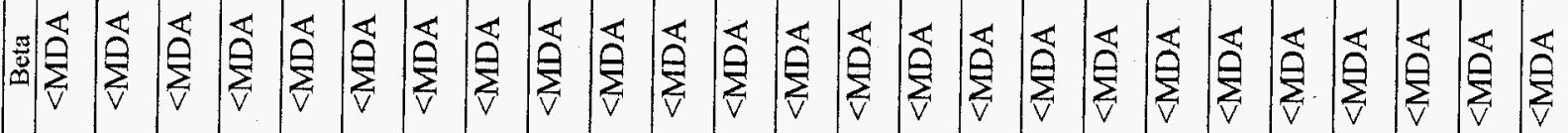

要

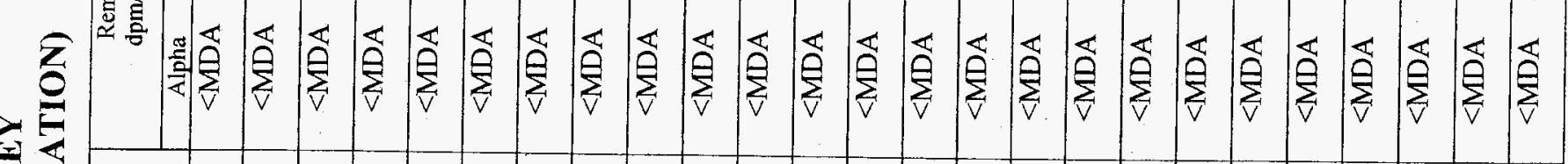

羟

要

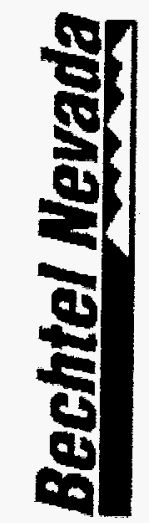


I

岁

$\checkmark$

藻

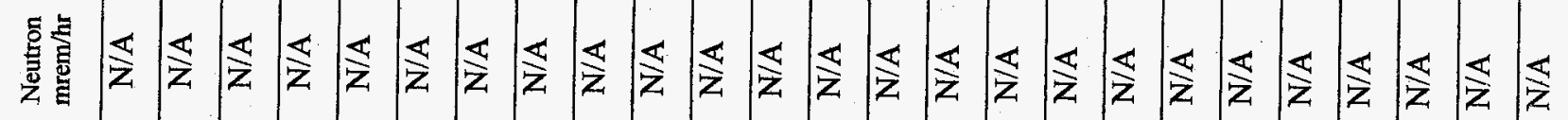

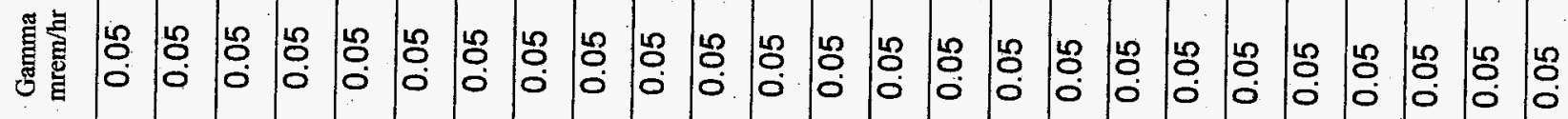

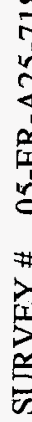

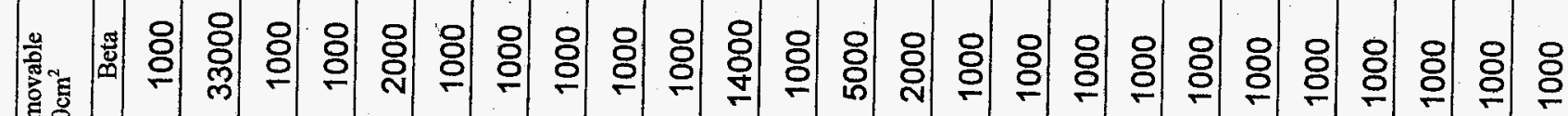

2

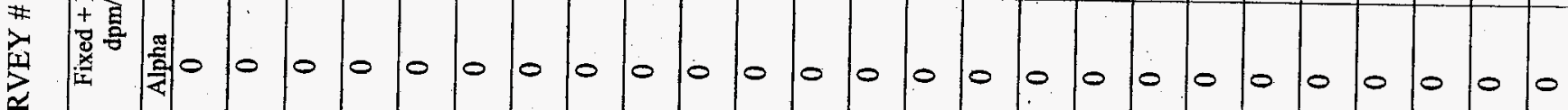

.

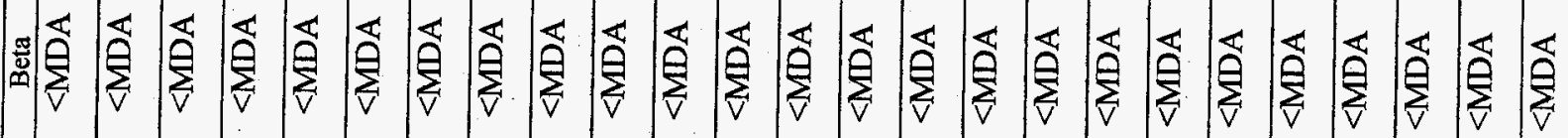

늘

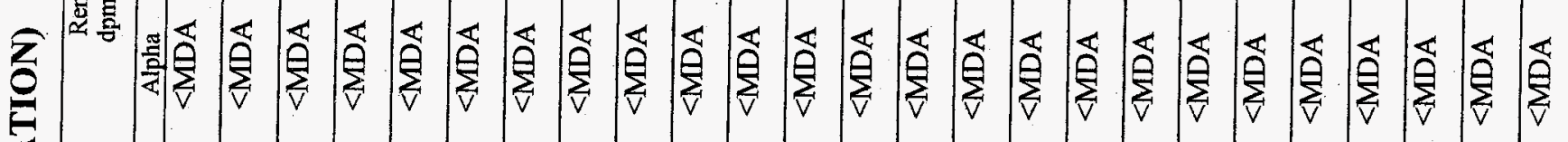

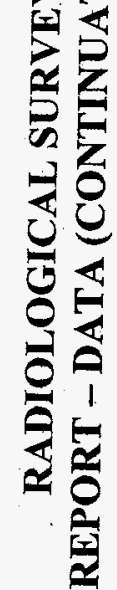

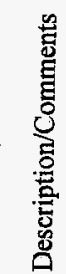

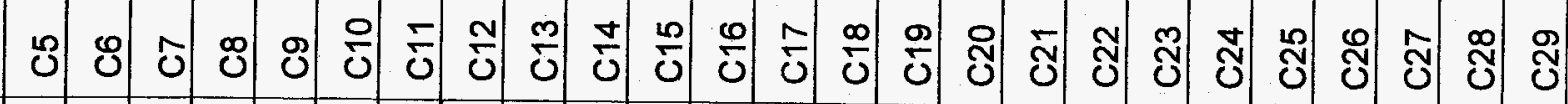

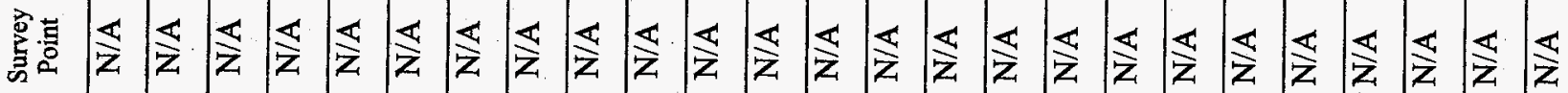




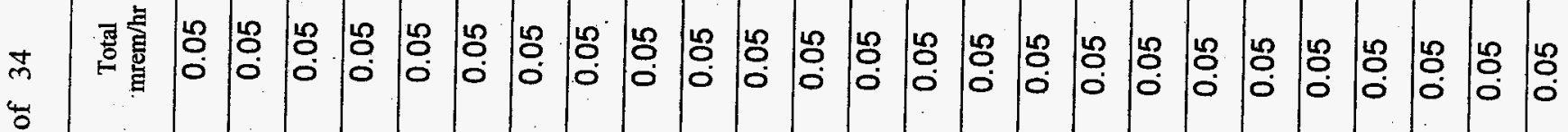

菭

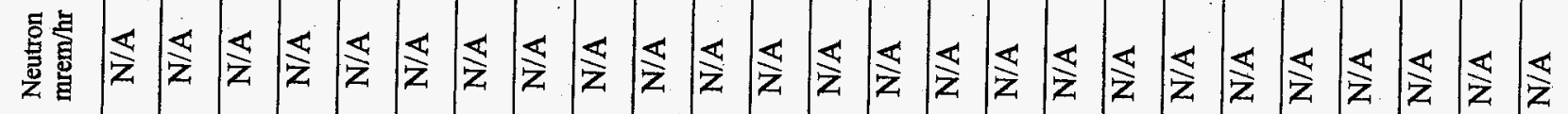

焉县

告

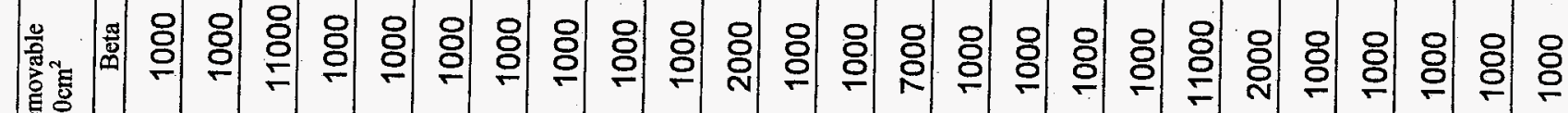

$+2$

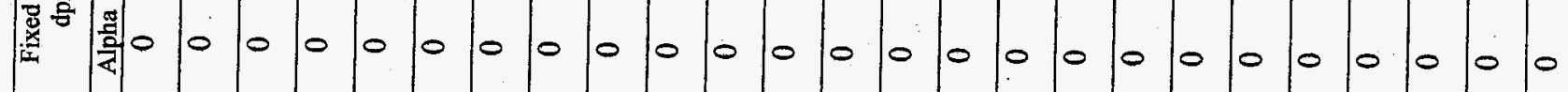

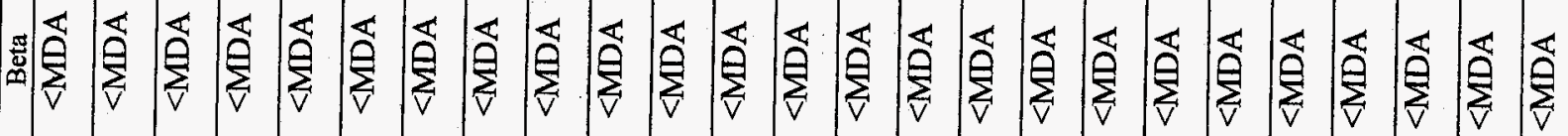

동

:

送

(2)

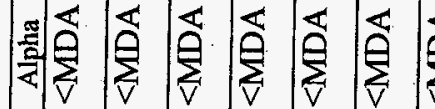

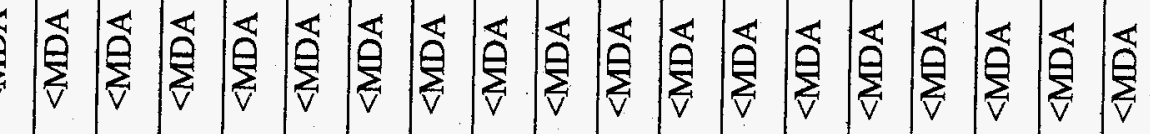

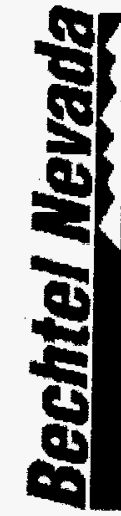

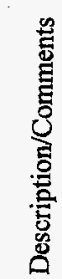

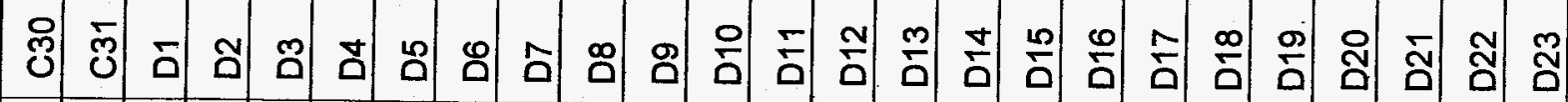

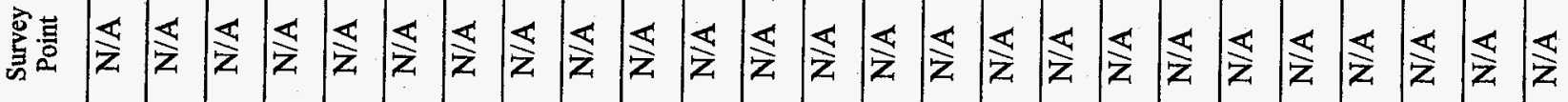




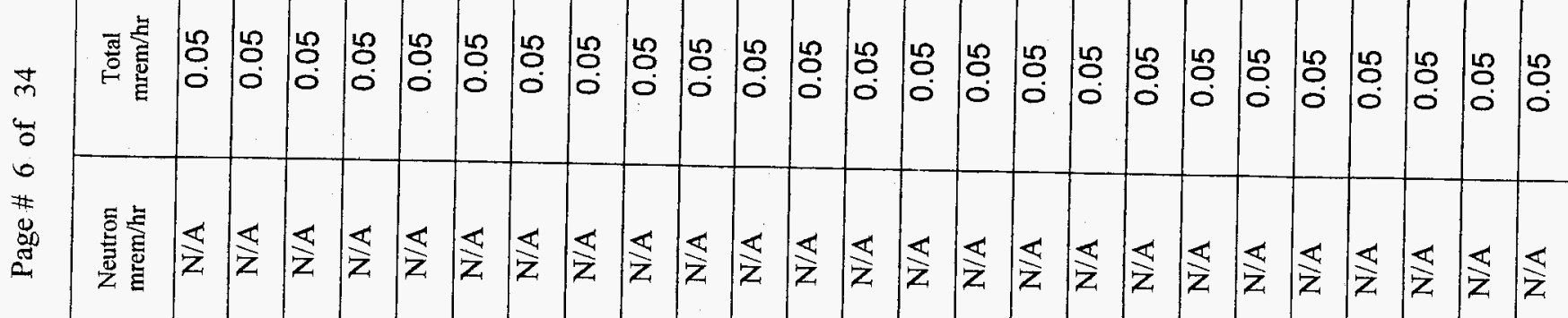

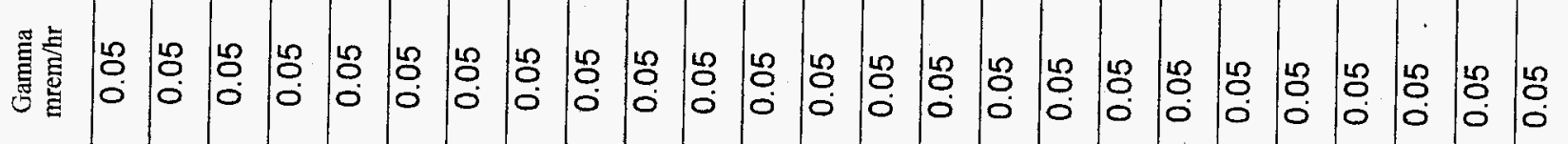

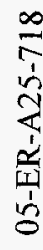

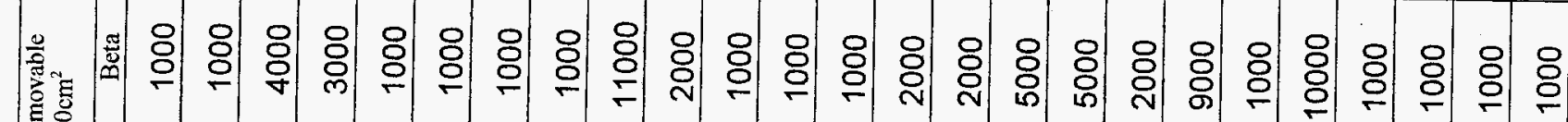
\%

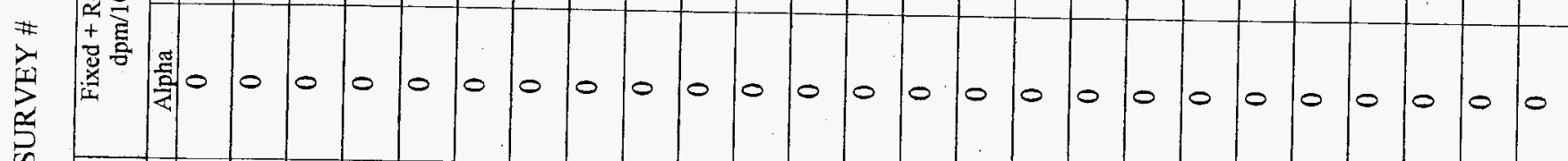

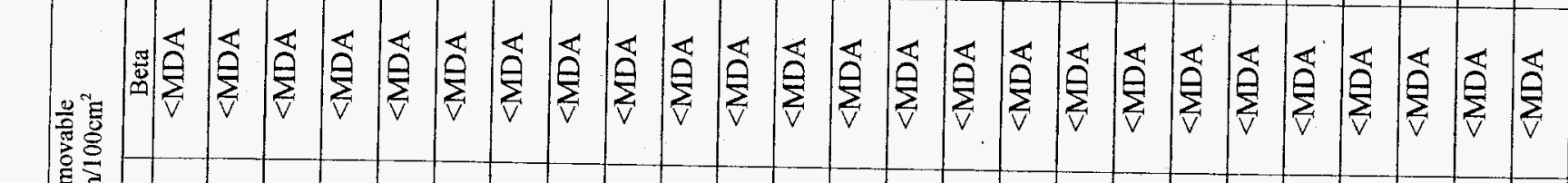

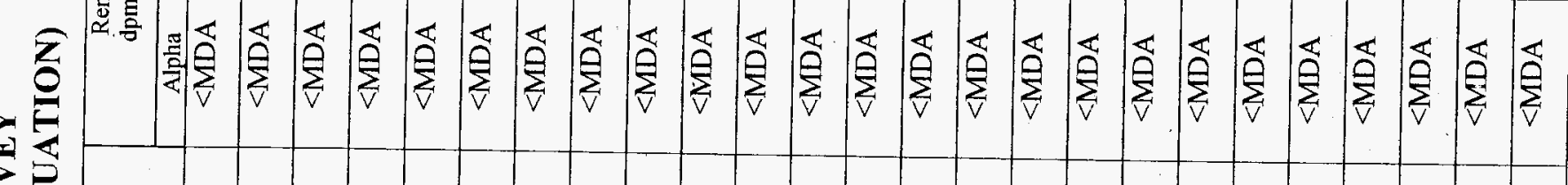
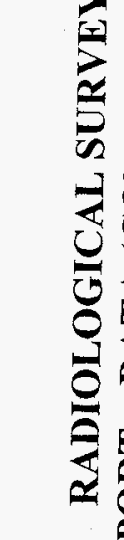

范

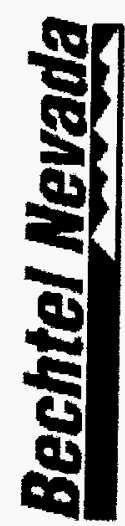

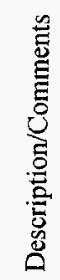

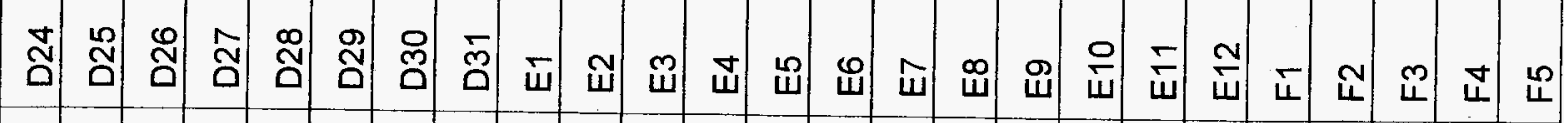

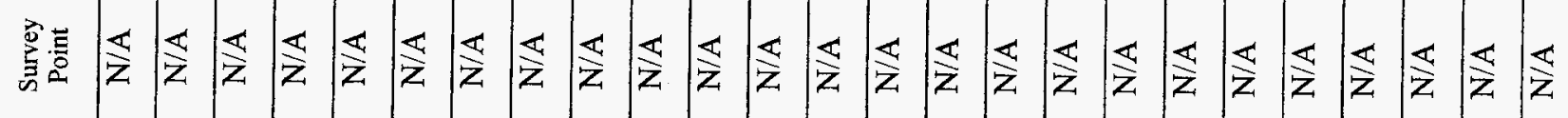




\section{苟

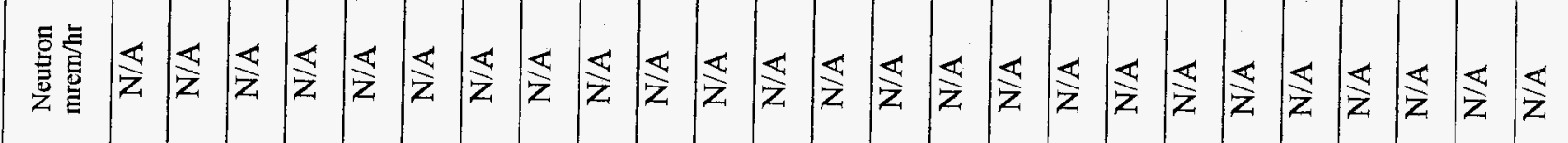

氞表

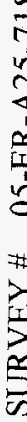

2 웅

* $+\frac{+}{\mathrm{g}}$

产

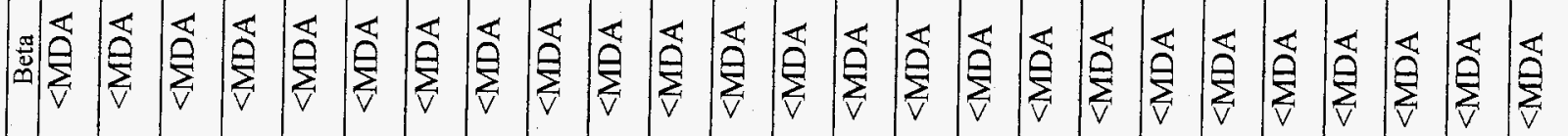

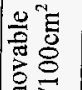

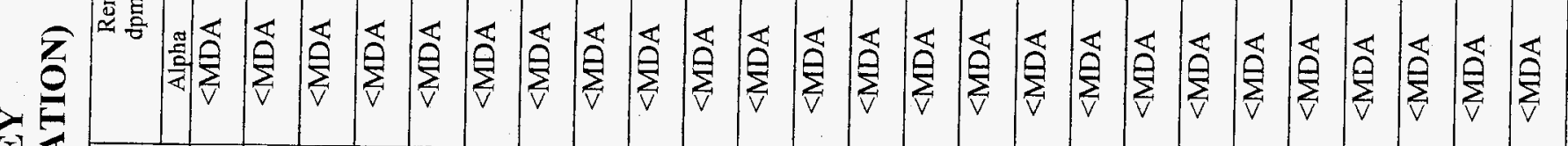

se

.

웅

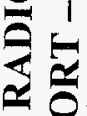

军

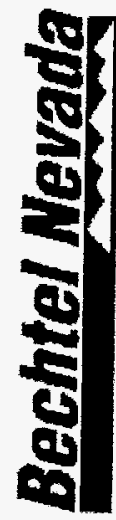

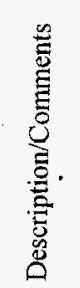

안 누

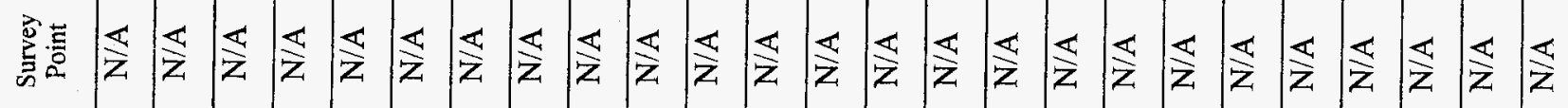




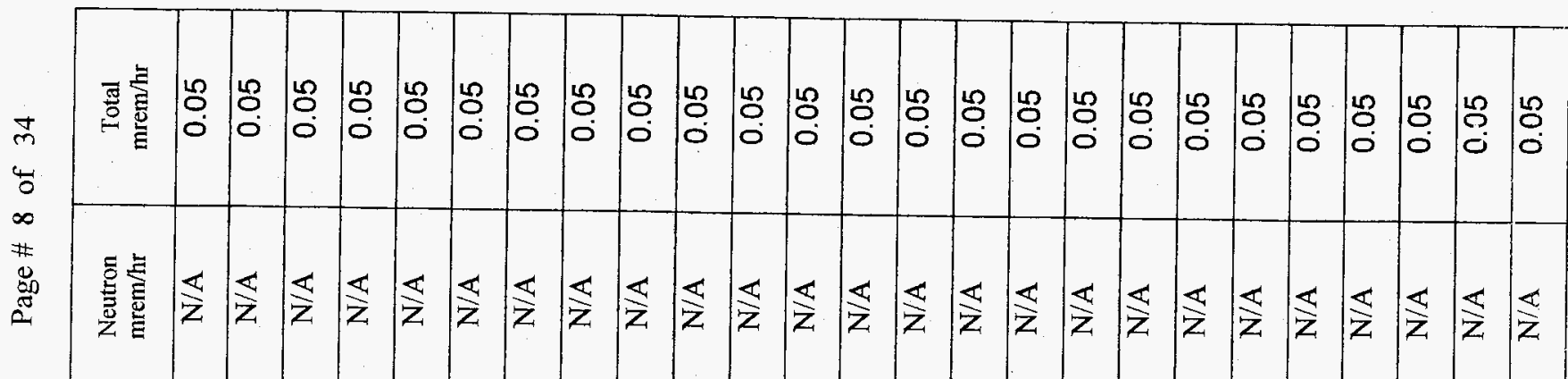

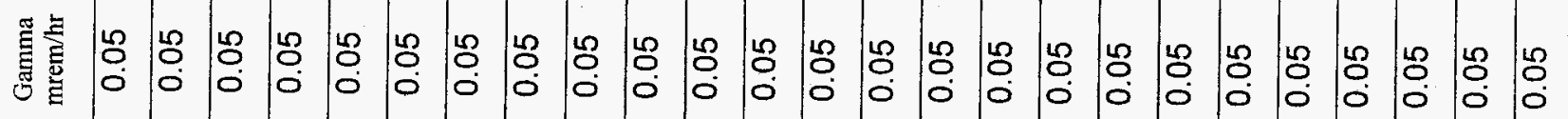

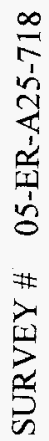

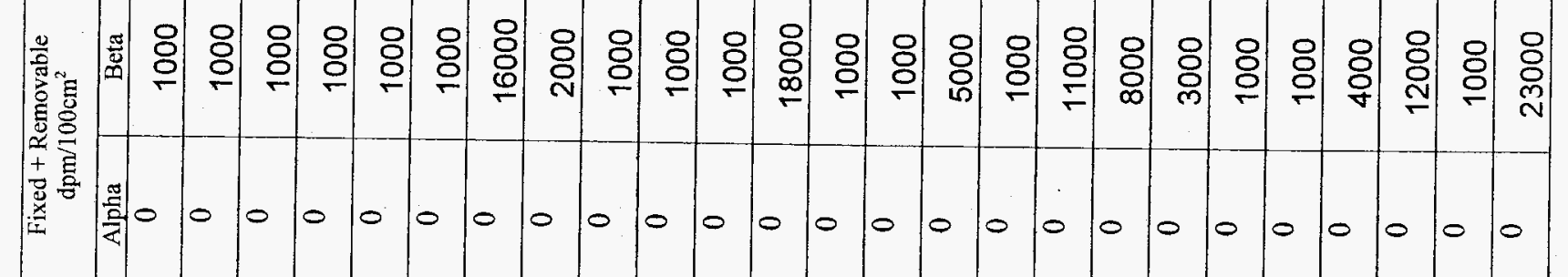

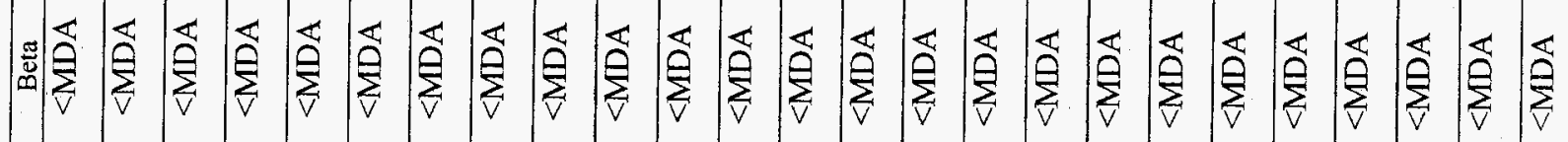
政

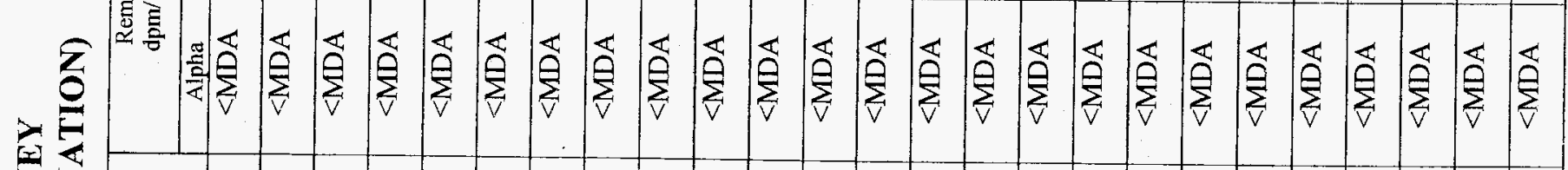

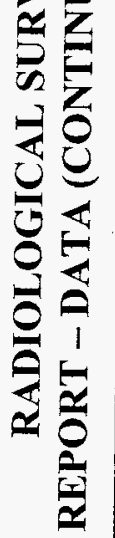

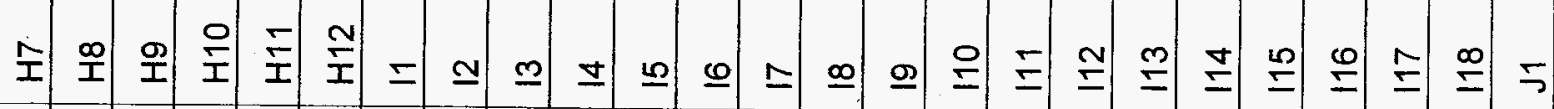
窵言 
吾婂 4

\#

棁

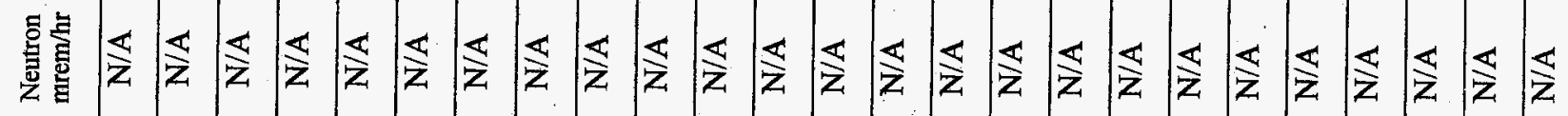

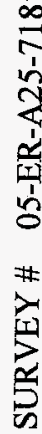

置

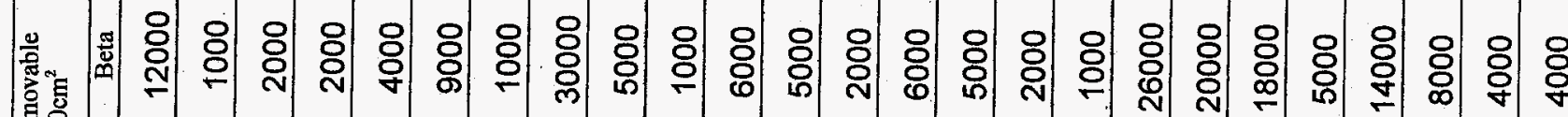

$\approx$

焉

0

0.

0.0

0.0

00

- 0

0

$\begin{array}{llllllllllllllllllllll}0 & 0 & 0 & 0 & 0 & 0 & 0 & 0\end{array}$

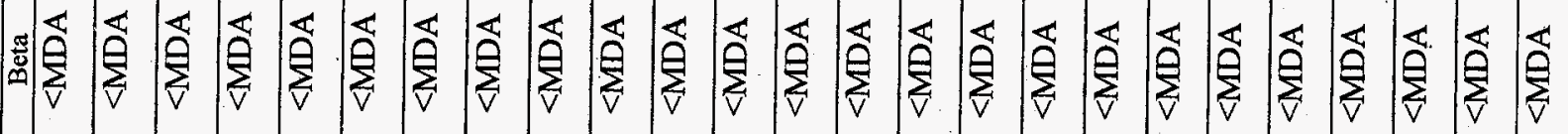

政

事高

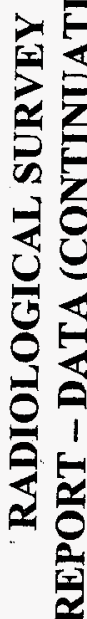

$\overbrace{0}$

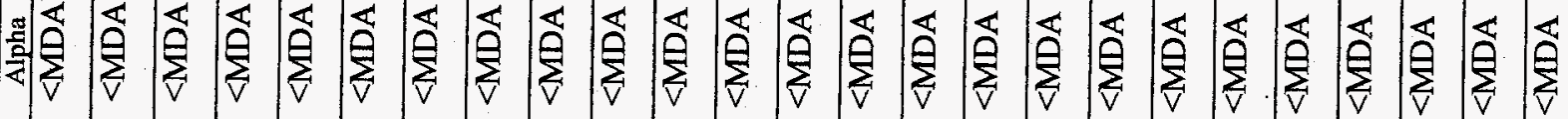

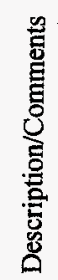

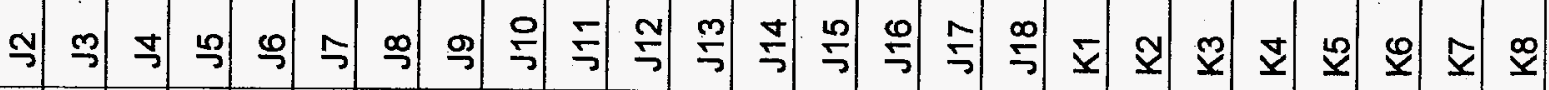

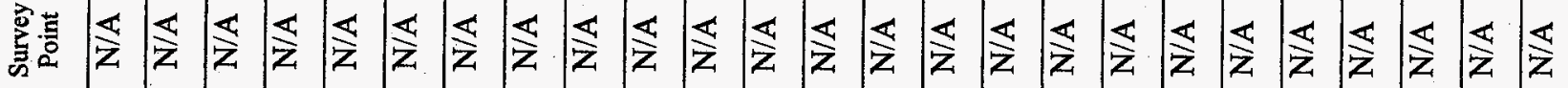




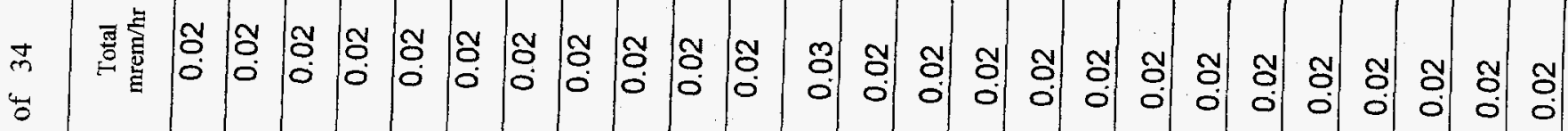

¿ั

(1)

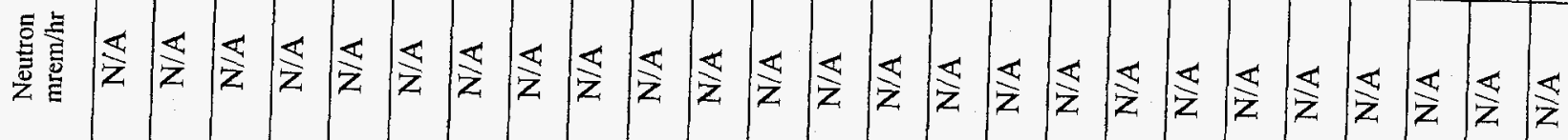



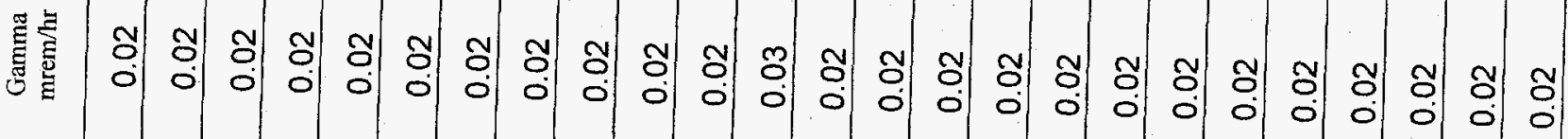

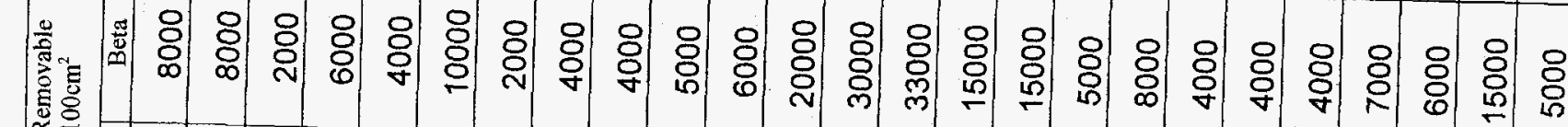

:

त

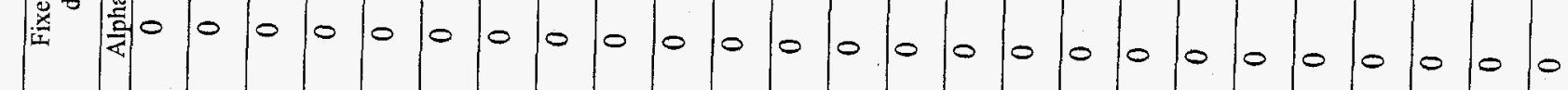

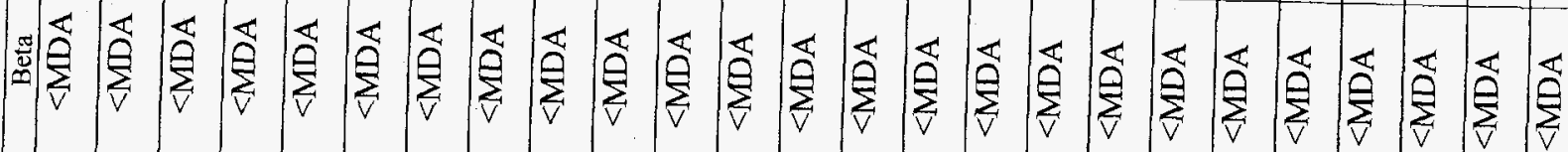

突

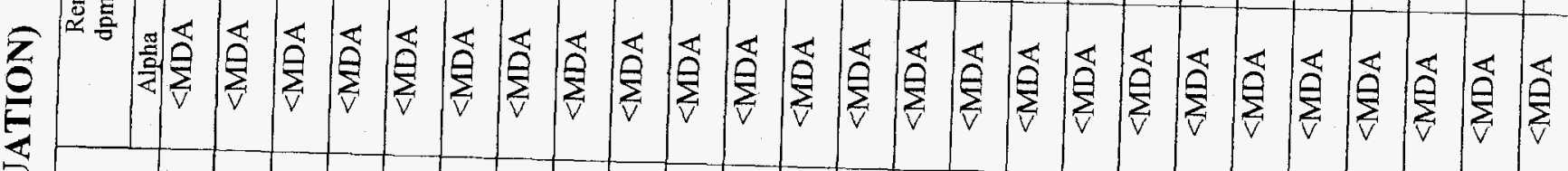

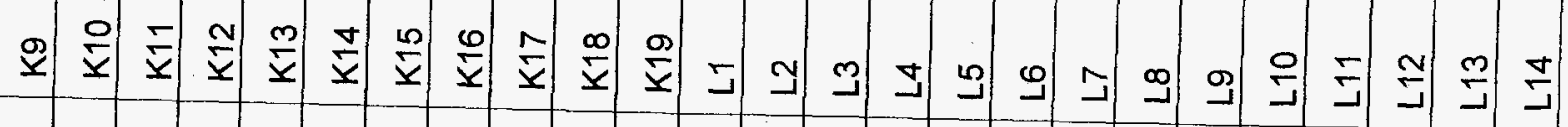

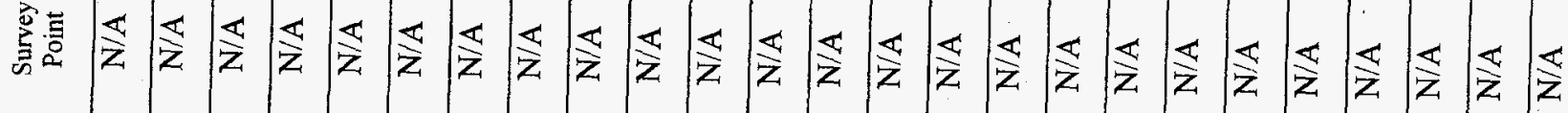




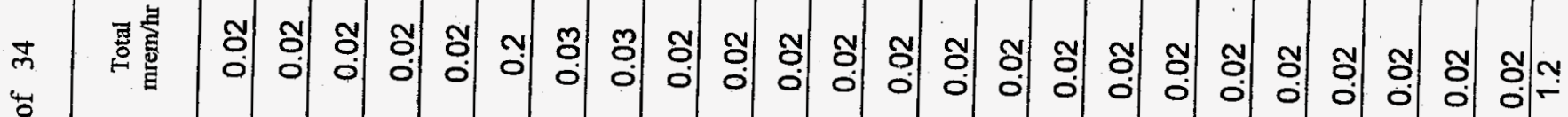

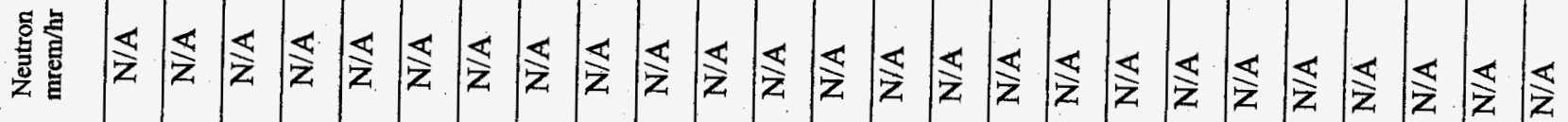

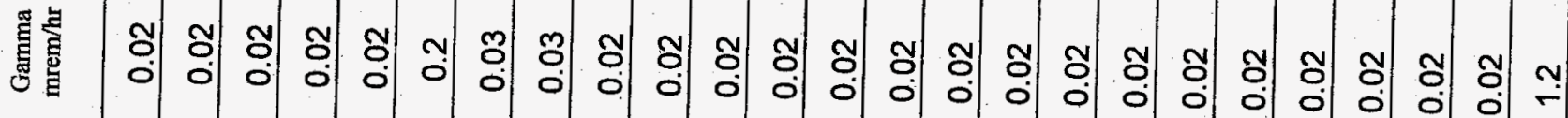

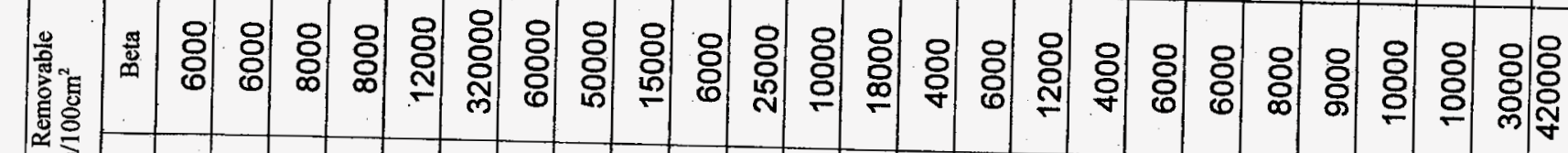

\#

究

들

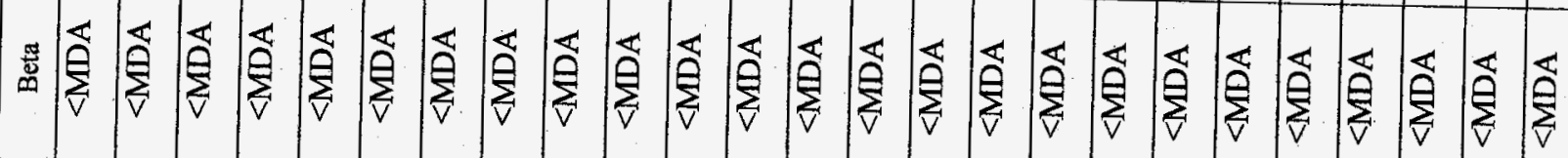

政

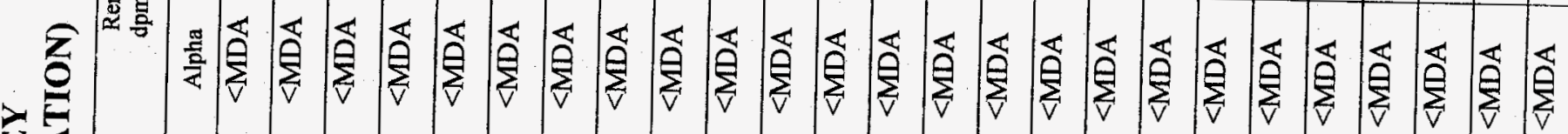

+ 행

홀

0

000

0.0

010

000

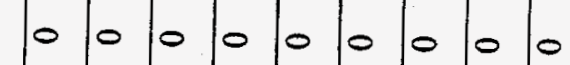


苦

$\cong$

范

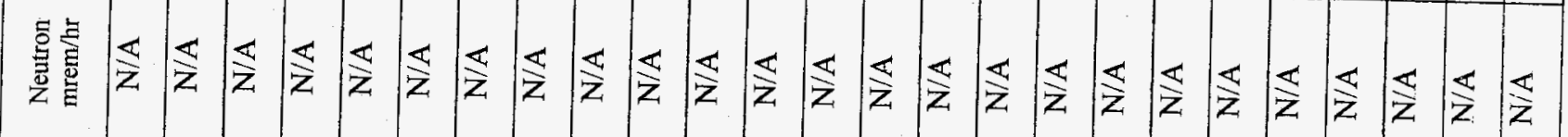

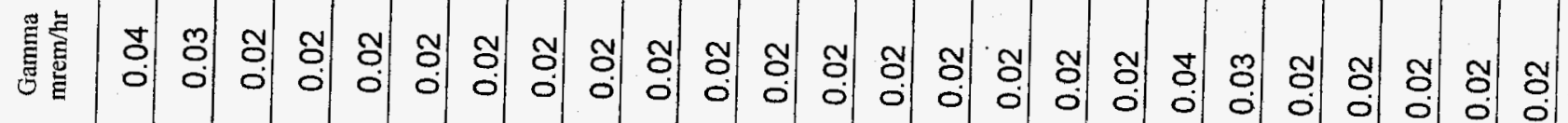

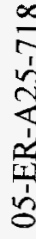

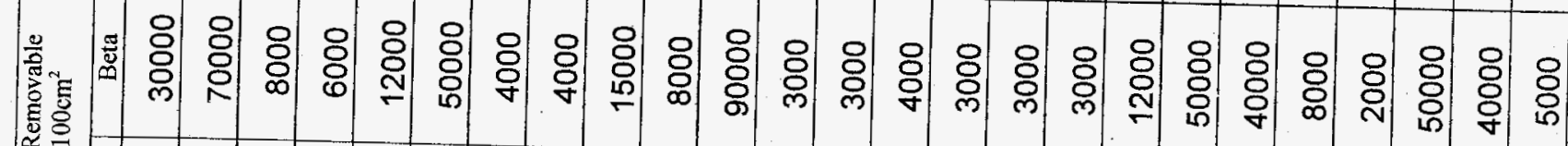

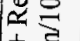

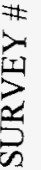

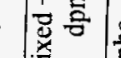

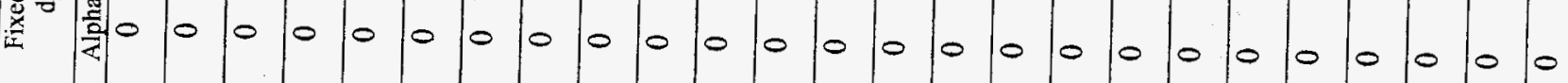

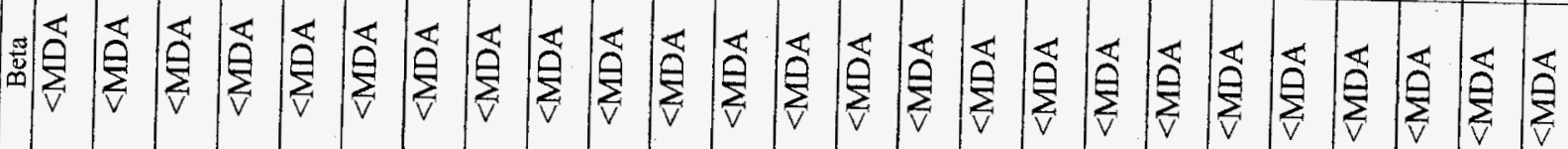

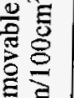

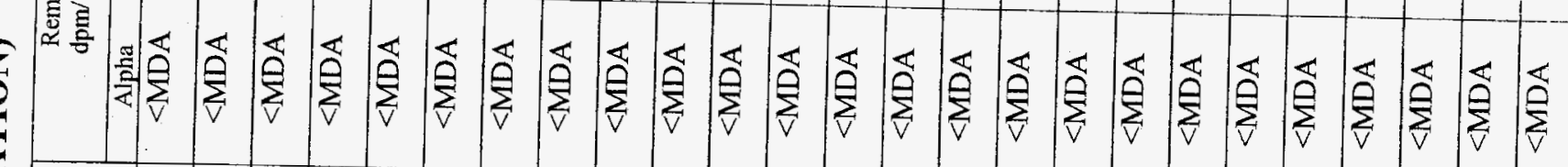

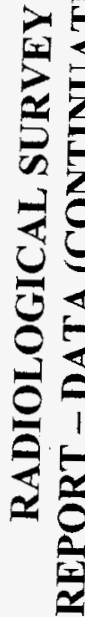

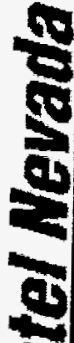

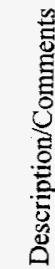

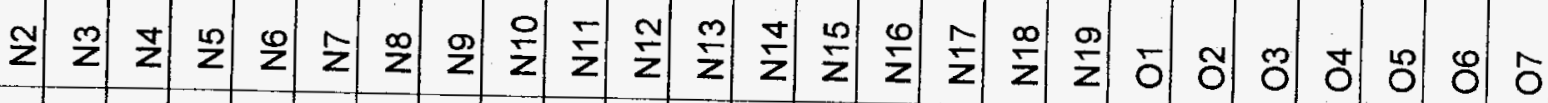

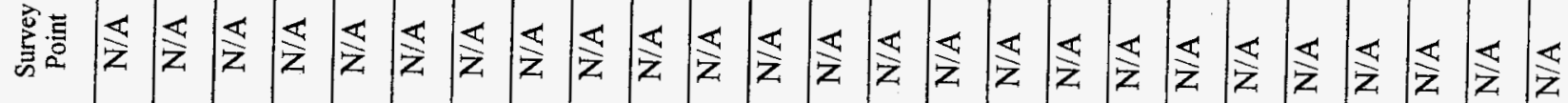




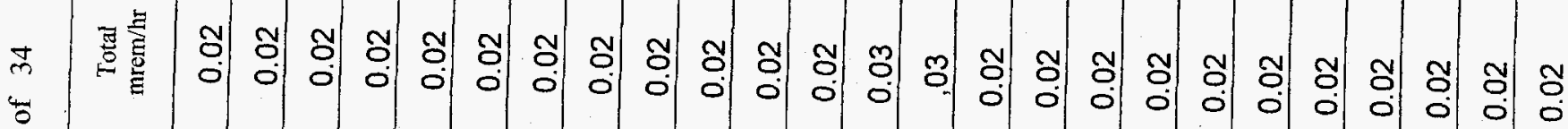

$=$

$\frac{\infty}{5}$

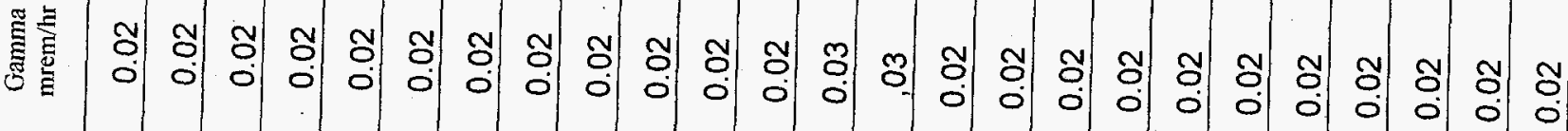

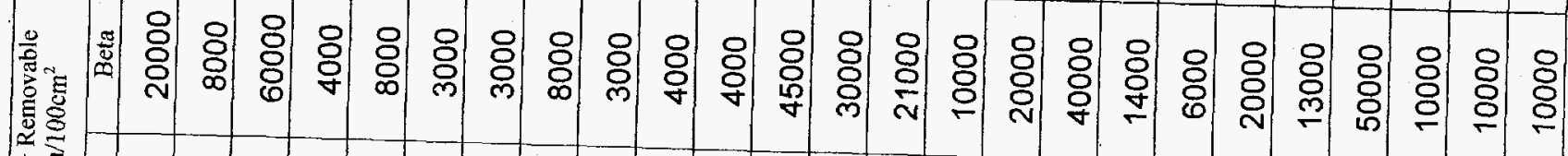

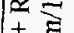

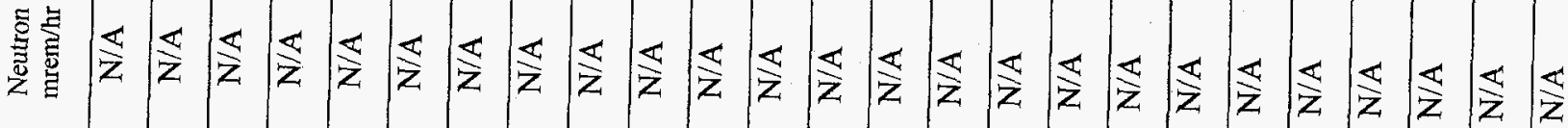

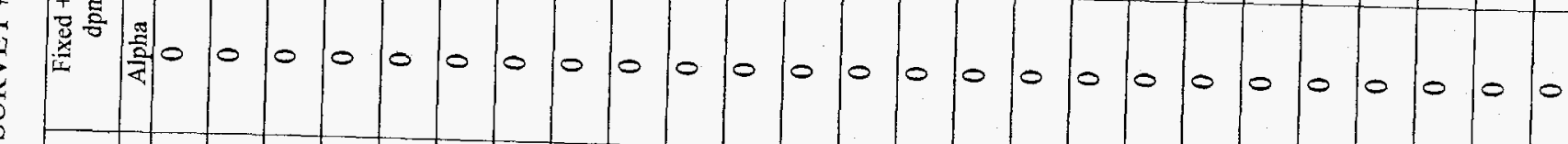

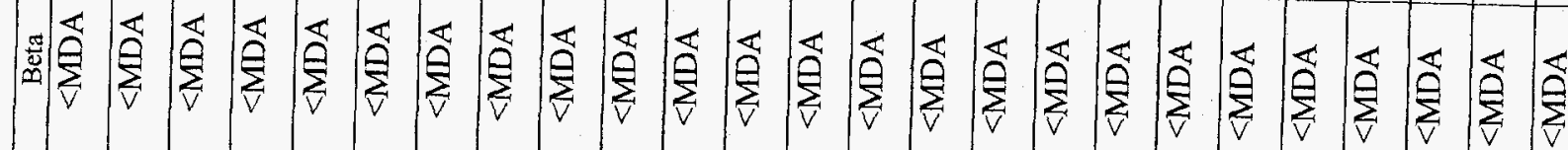

है

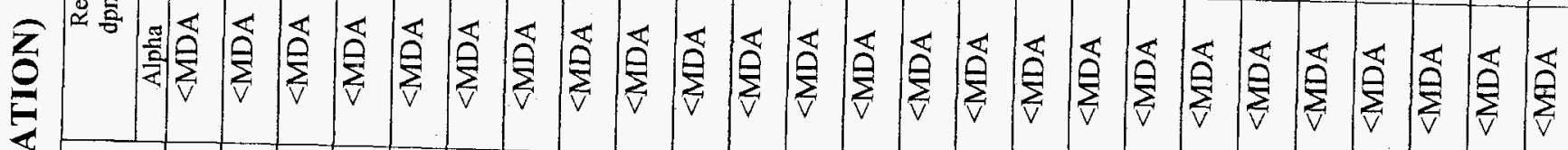

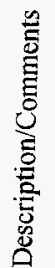

o : g

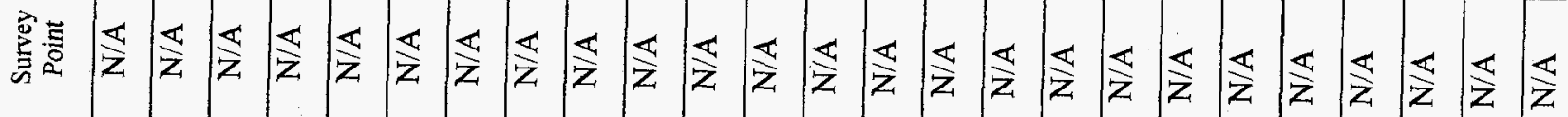




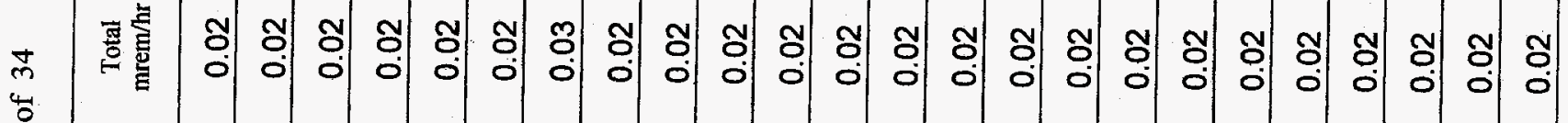

$\pm$

范

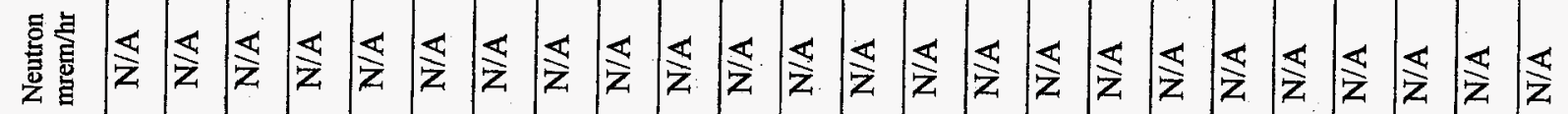

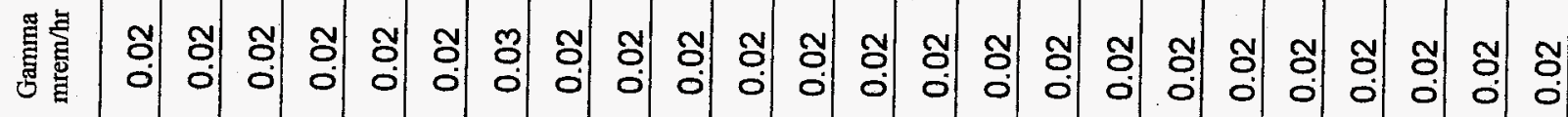

ڤั)

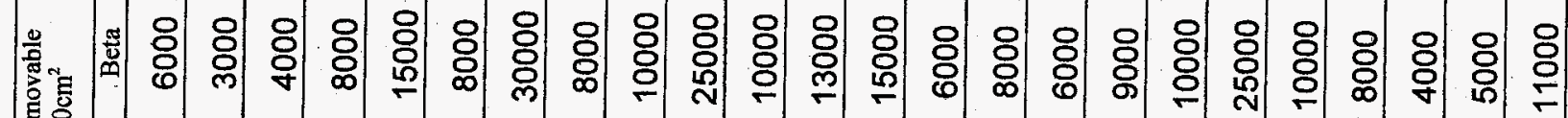
$\approx$

+ 흥

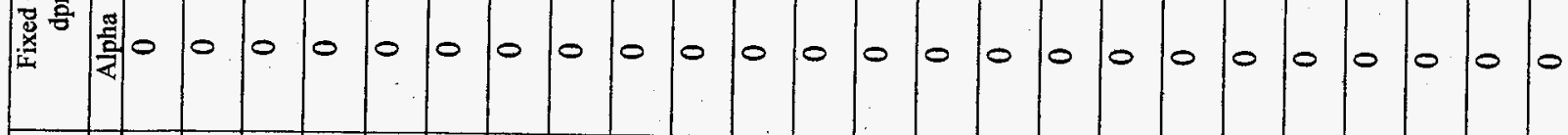

चี

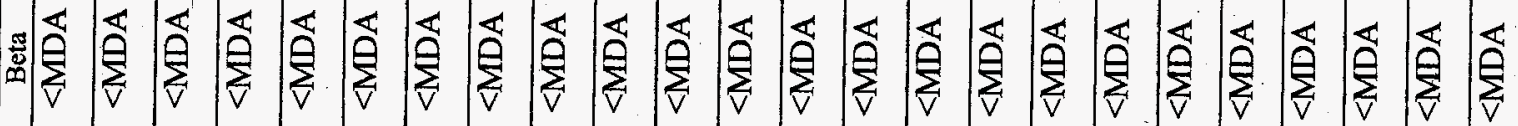

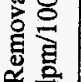

2

$>7$

$\approx \frac{8}{2} \ll$

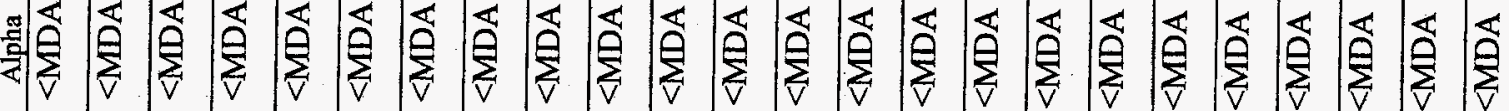

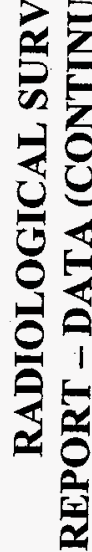

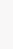

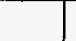




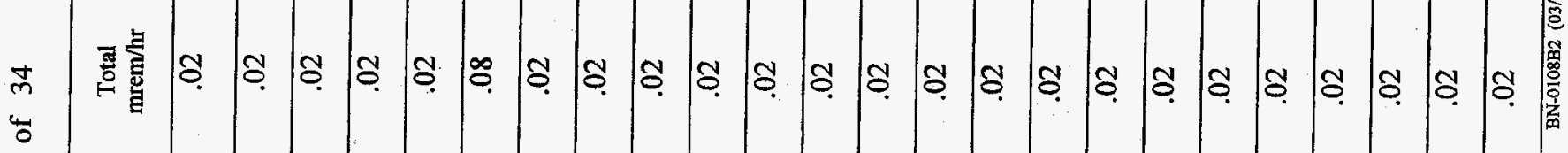

n

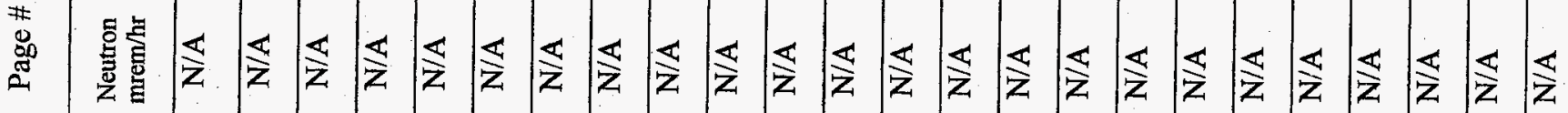

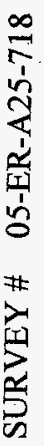

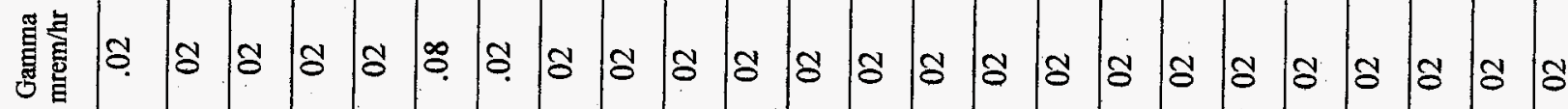

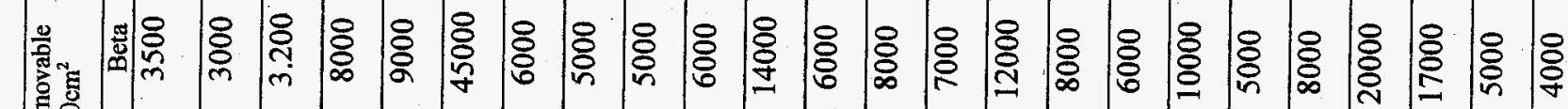
$+\underset{1}{+}$

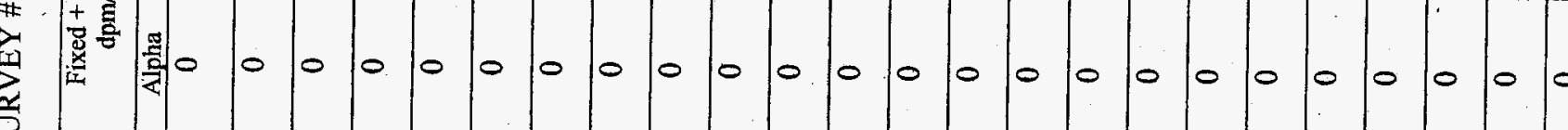

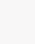

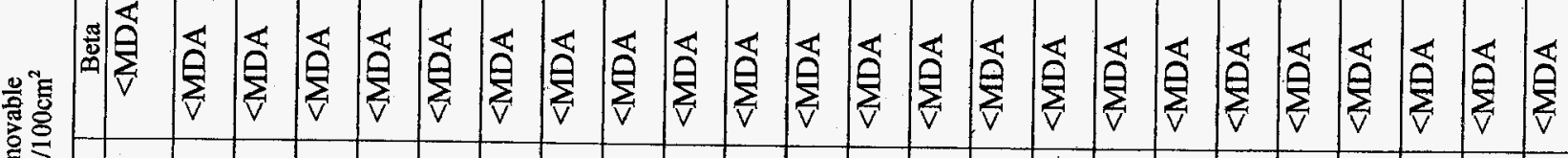
.
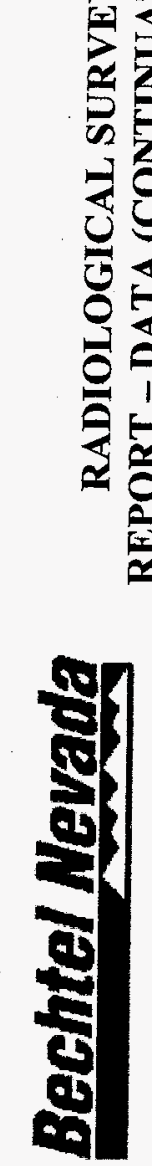

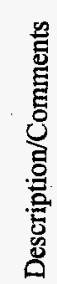

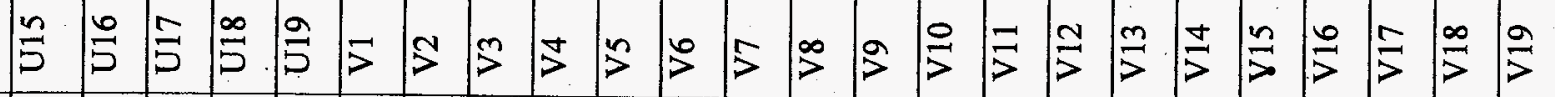

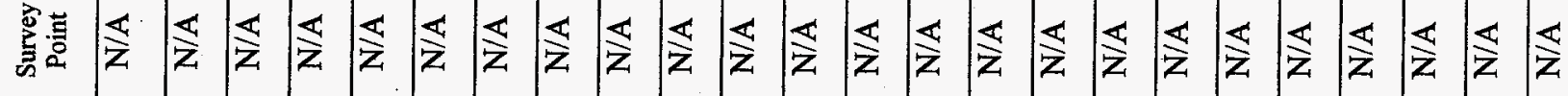




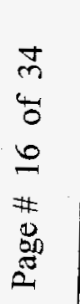

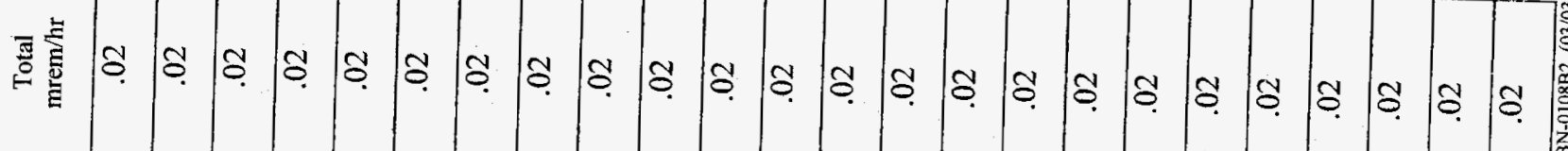

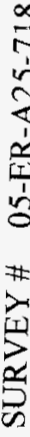

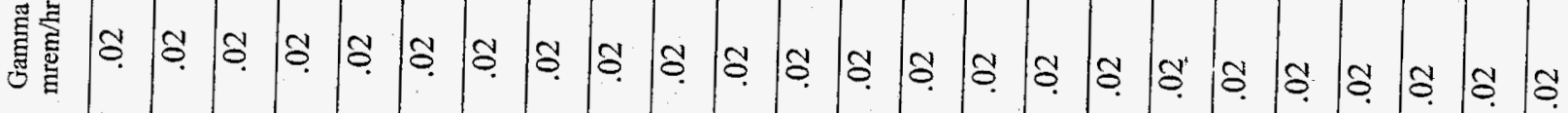

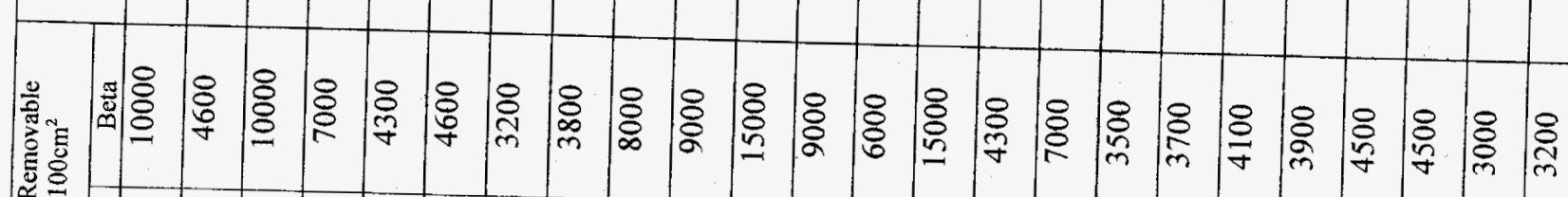

政

窟

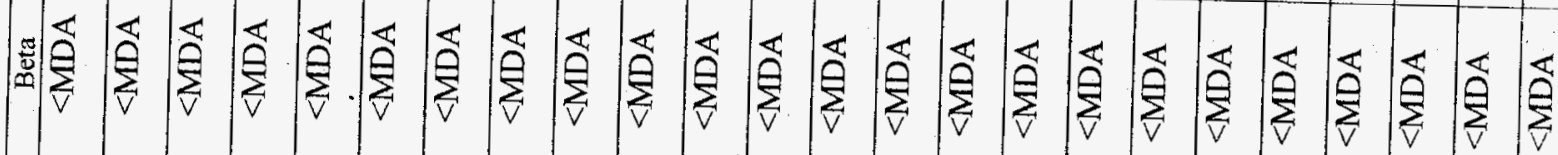

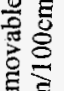

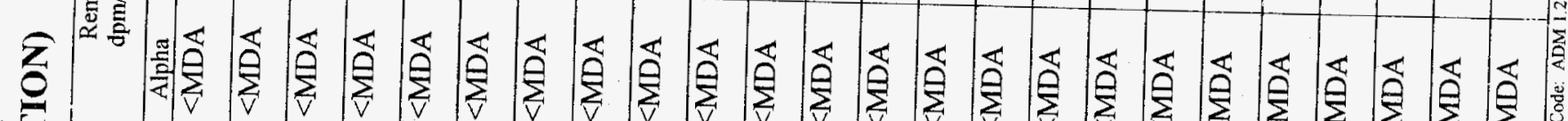

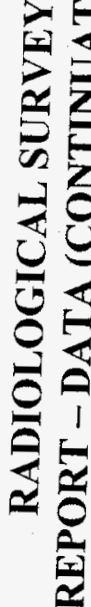

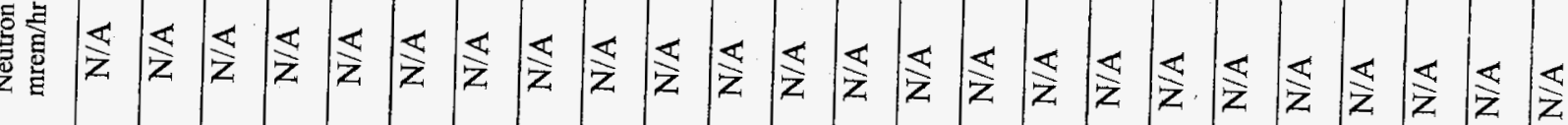

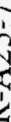




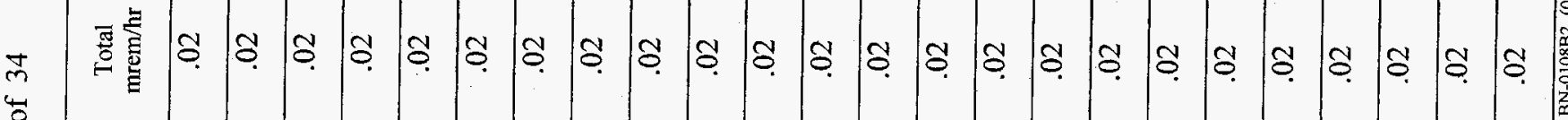

=

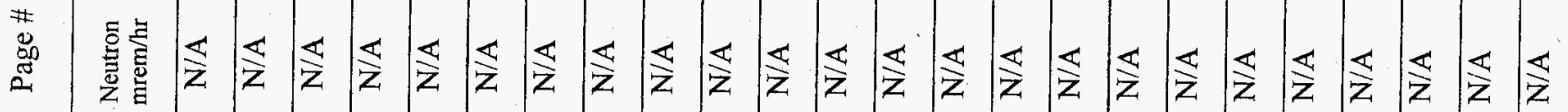

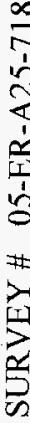

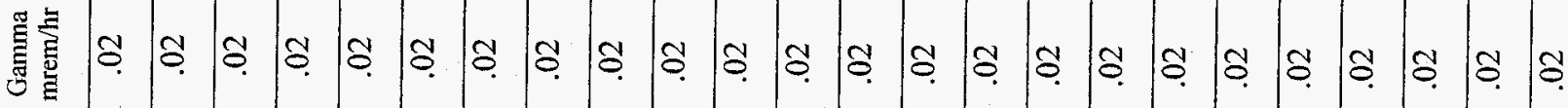

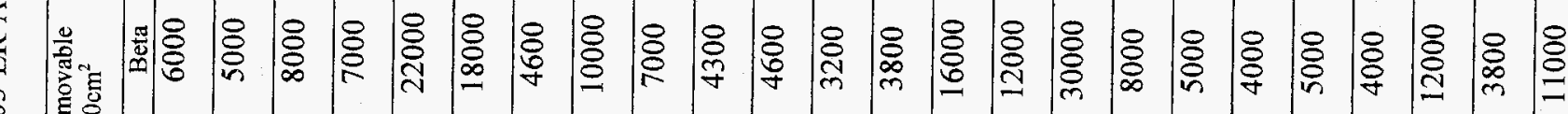

象

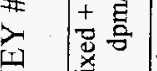

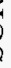

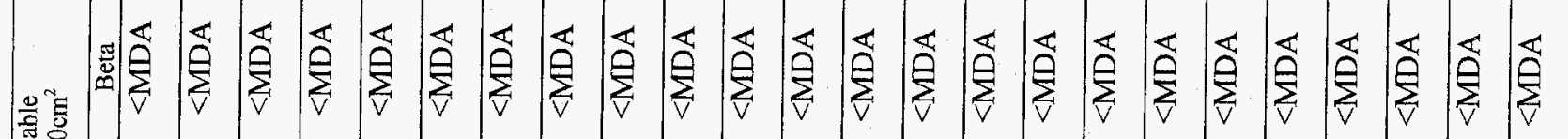

응

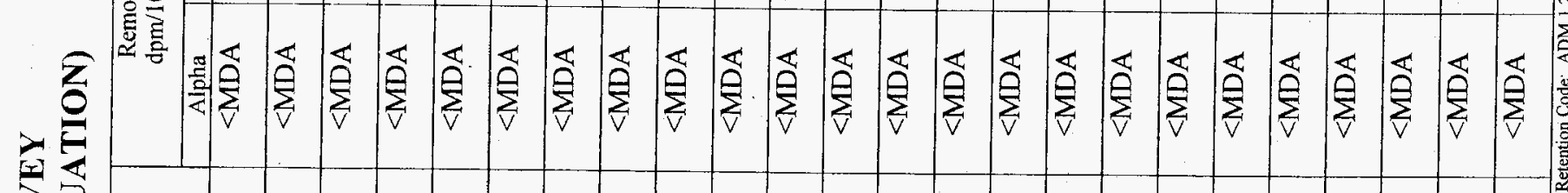

要

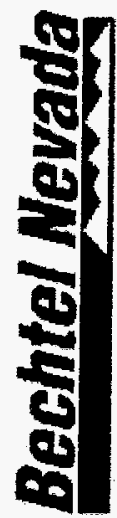
원

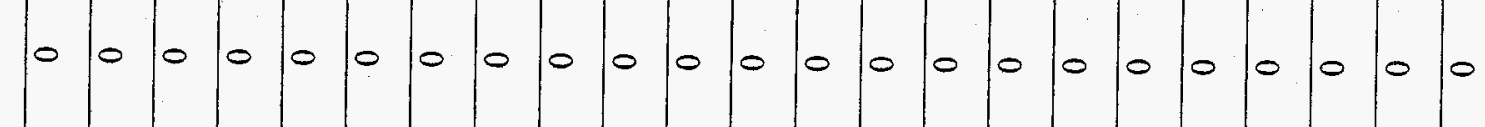




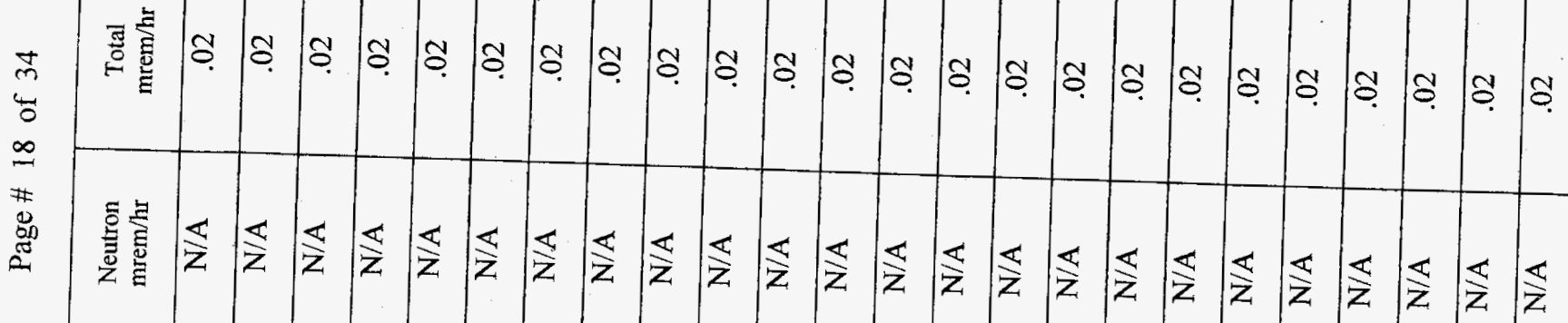

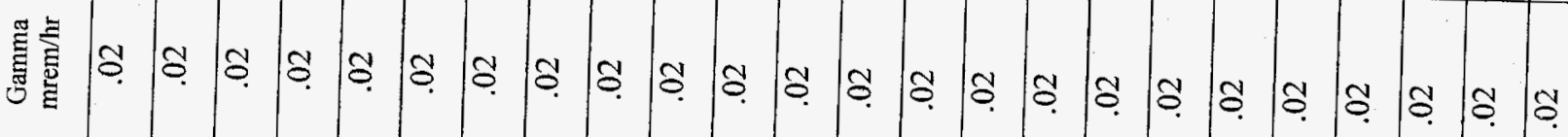

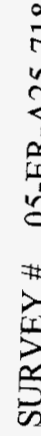

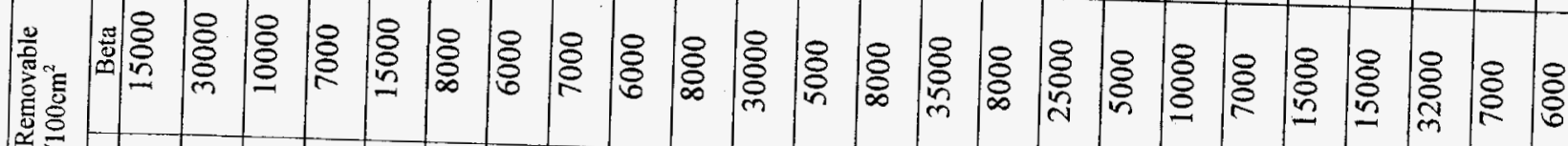

旁

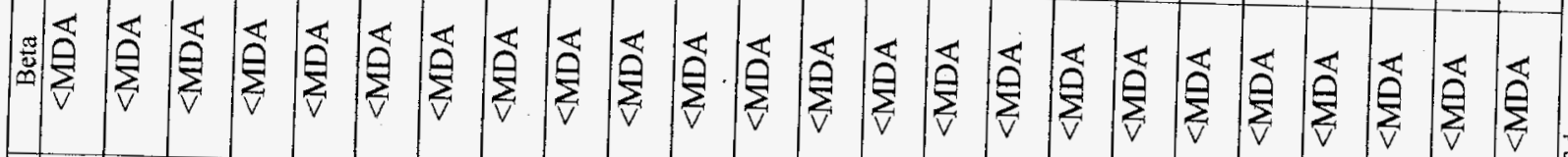

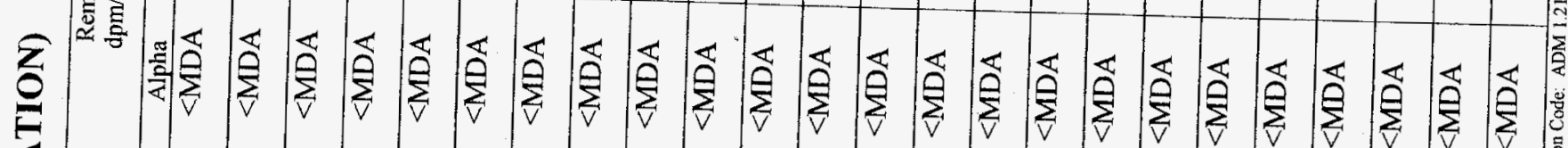

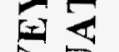

ํㅗㄹ

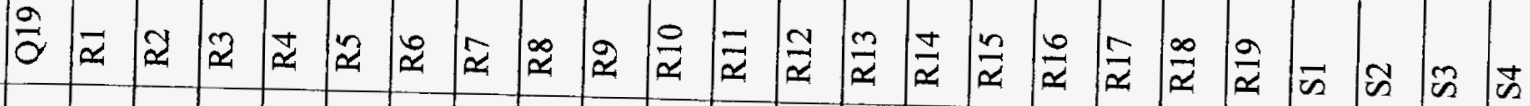

空言 


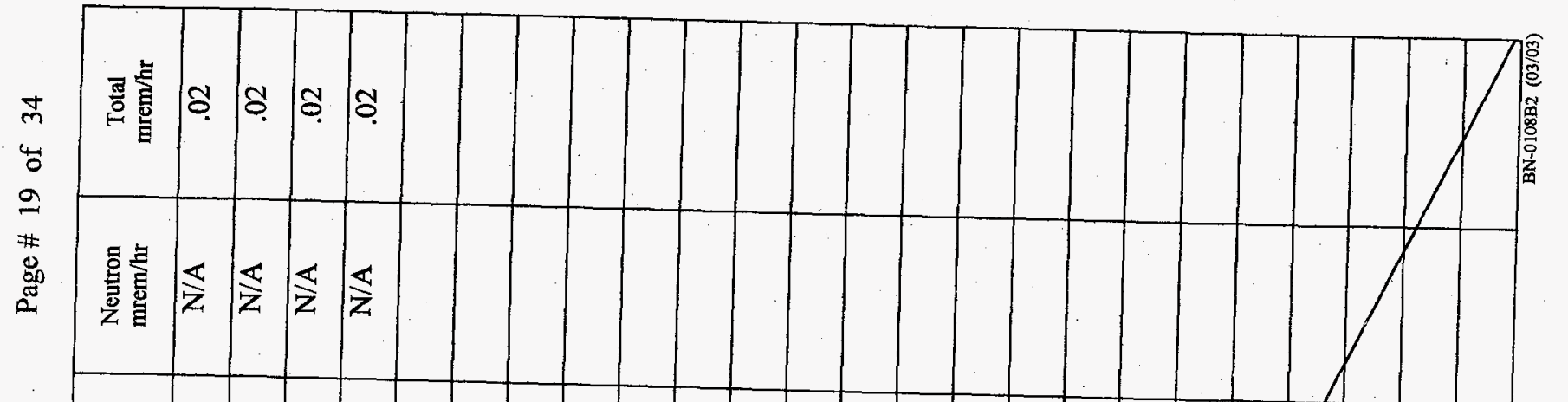

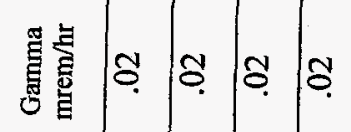

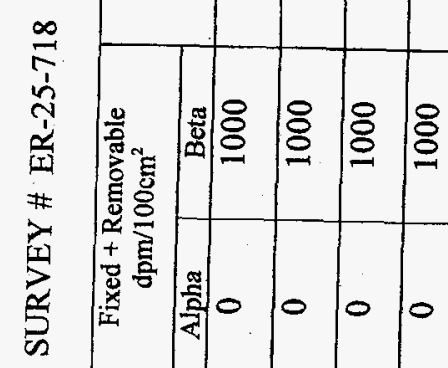

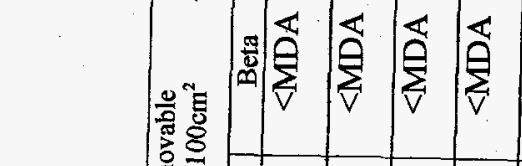

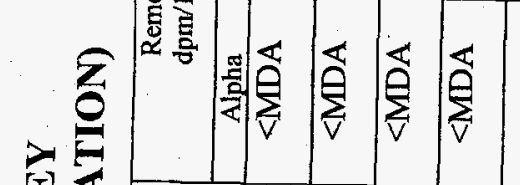

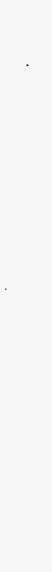

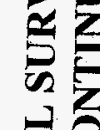

,

s.

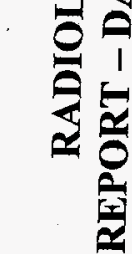

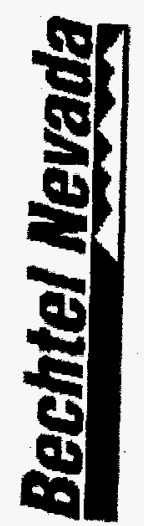

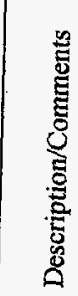

응 을 을

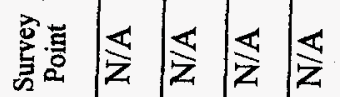


SOnUEG OS-ERA25-T18

Page zo or 34 
Sonves O5-ER-A25-718 PAGE 21 of 34 


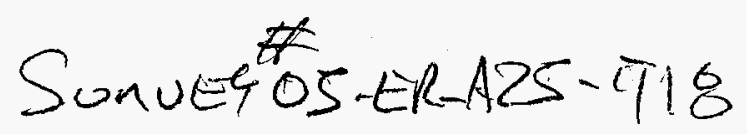

Page 22 of 34

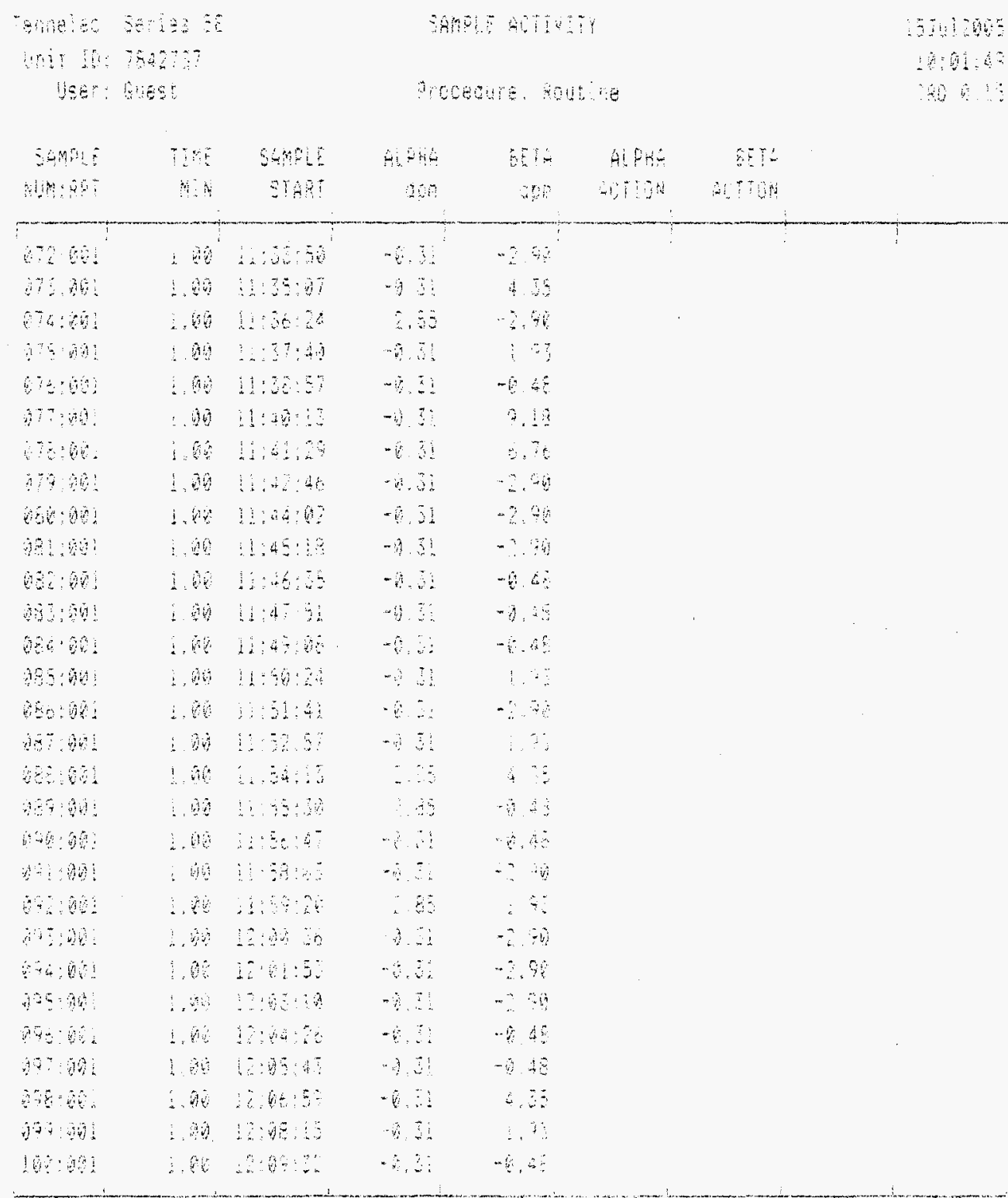

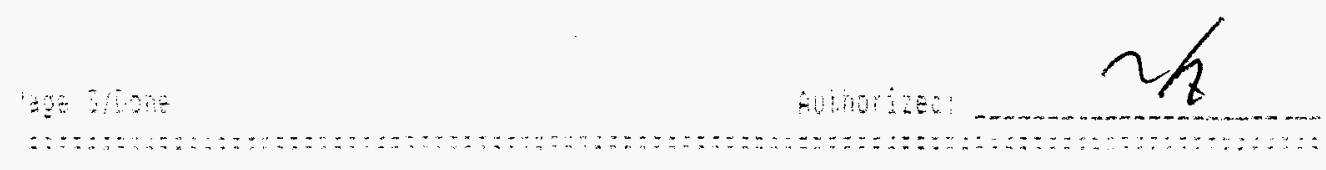


SUNUEYOS-ER-A25-918 Page 23 o 34

enteds sertes 5

Unit In: 7842137

User: Gues:
BAMPIE COTHET!

procenure houtire

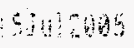

22.35 .45

600.15

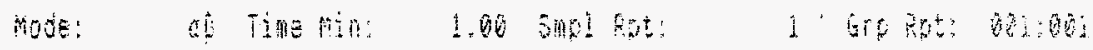

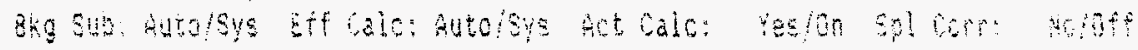

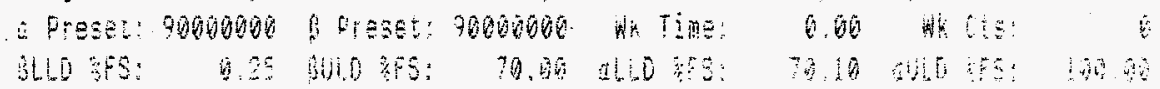

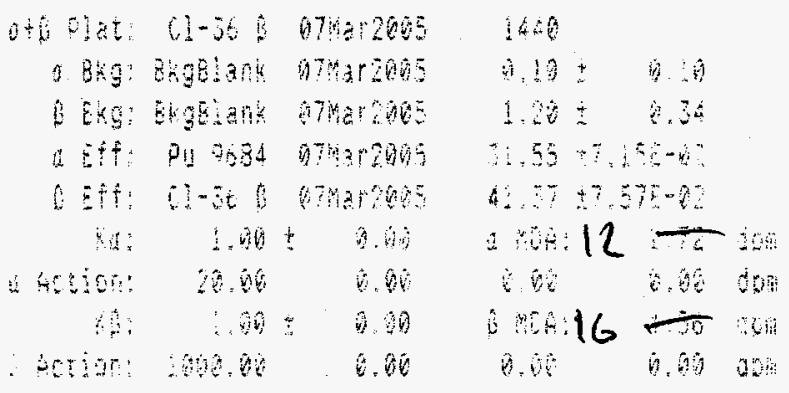

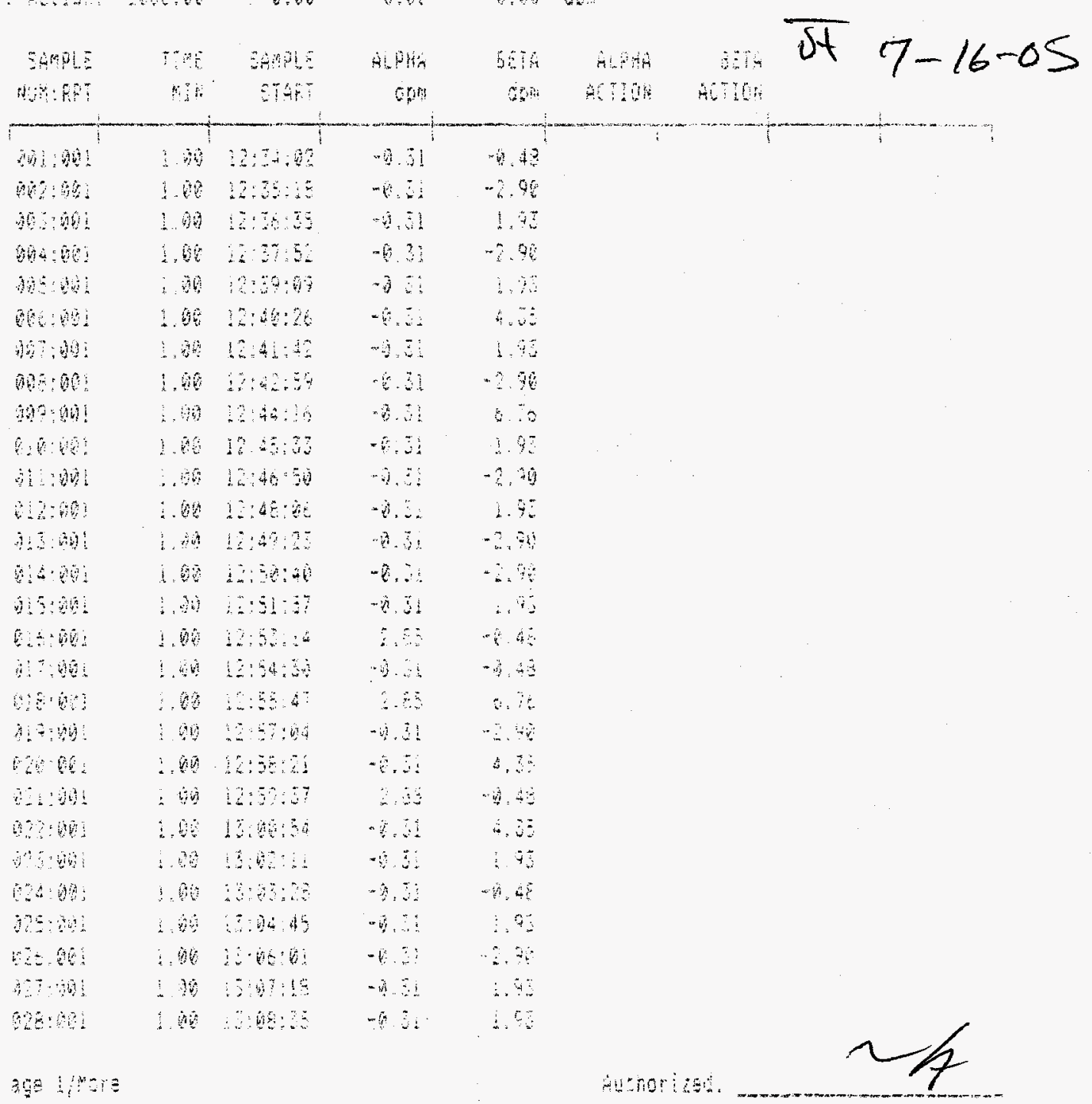


Bntaise Sertas 58

4n: If: 724275:

User: Gues:

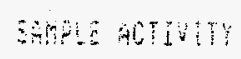

15012965

Procedure: Fontine
$12: 33: 49$

6.0 0.5

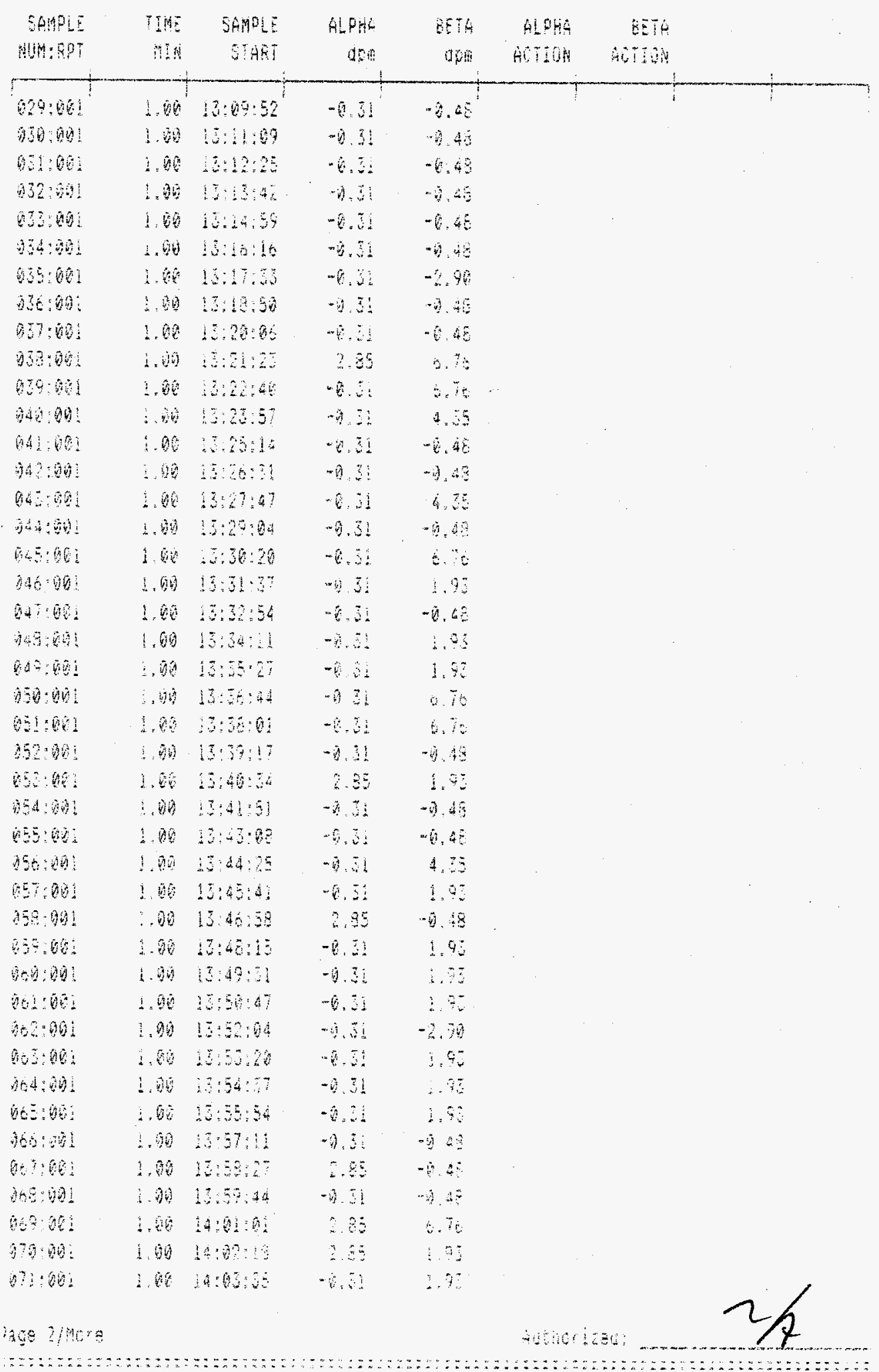




$$
\begin{gathered}
\text { SOMUEEOS-ER-A25-1718 } \\
\text { PAGE } 25 \text { of } 39
\end{gathered}
$$

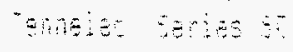

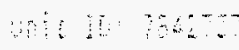

teas atos:
$5090894:$

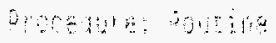

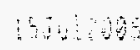

19:3:45

बति :

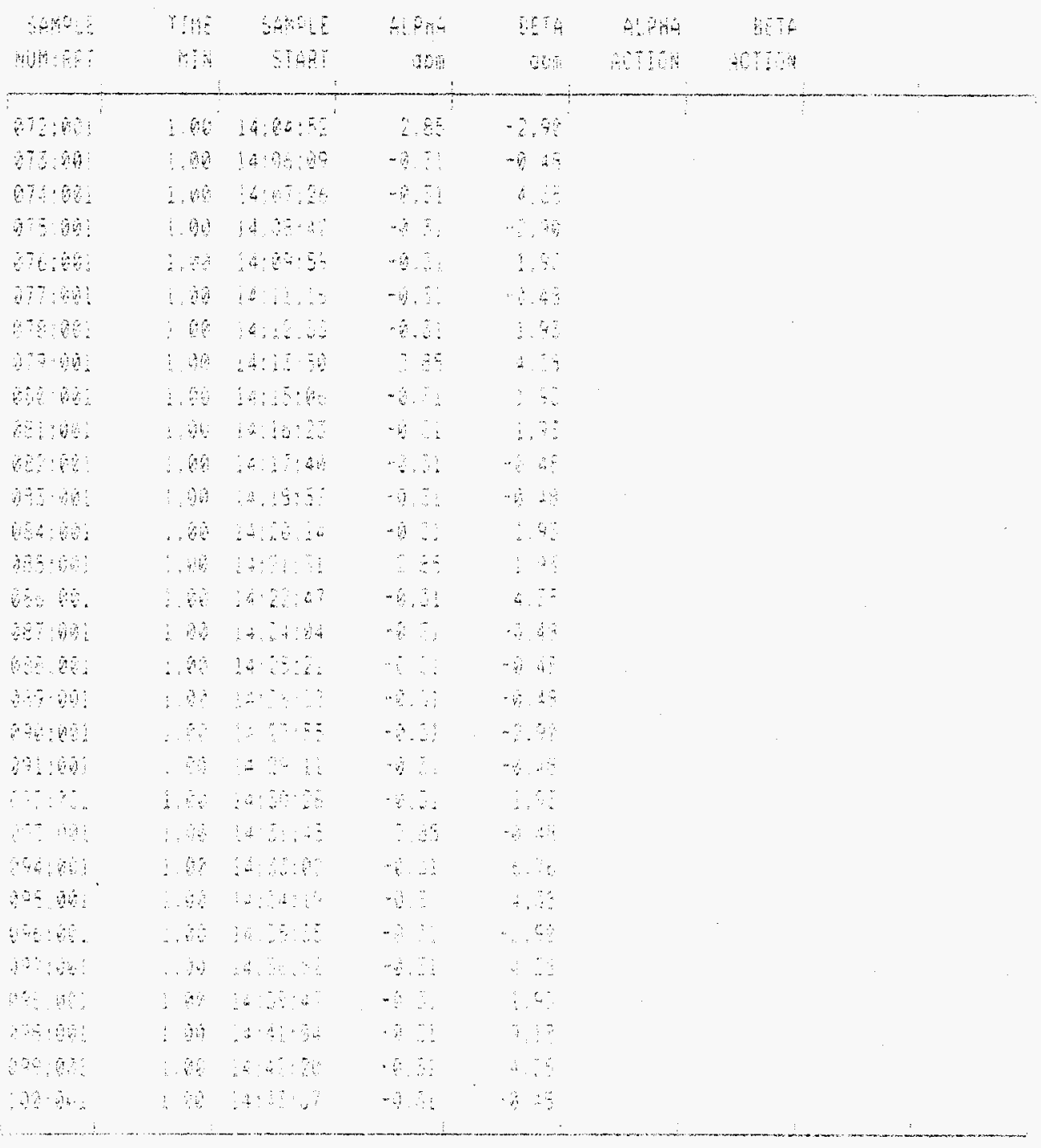

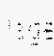

Th 
SUMUEY OS-ER-A25-918

PAge 26 of 34

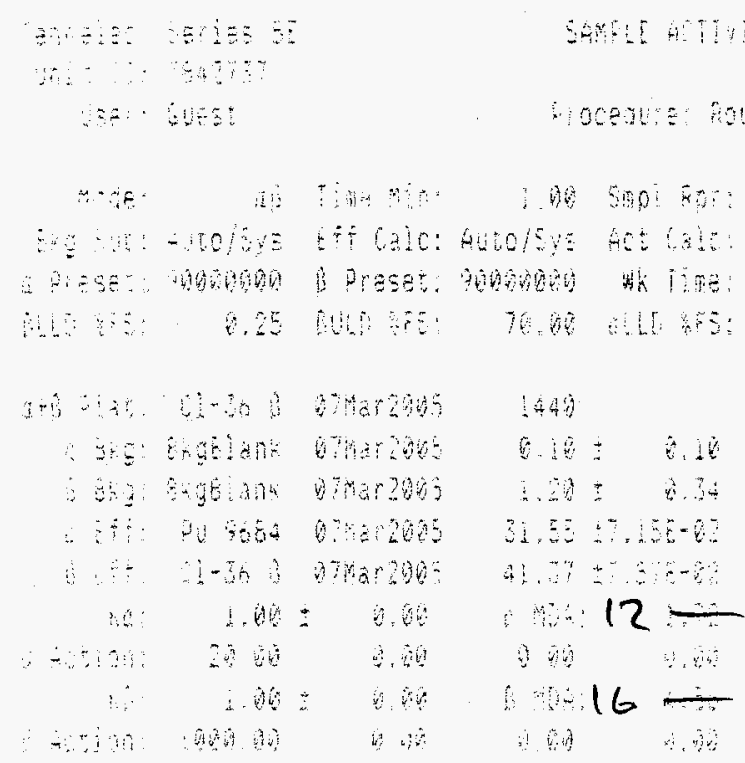

Bution

A. $13 . \mathrm{s}$

CA $1: 1$

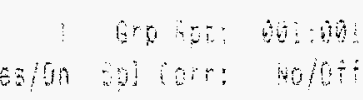

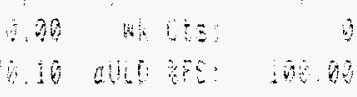

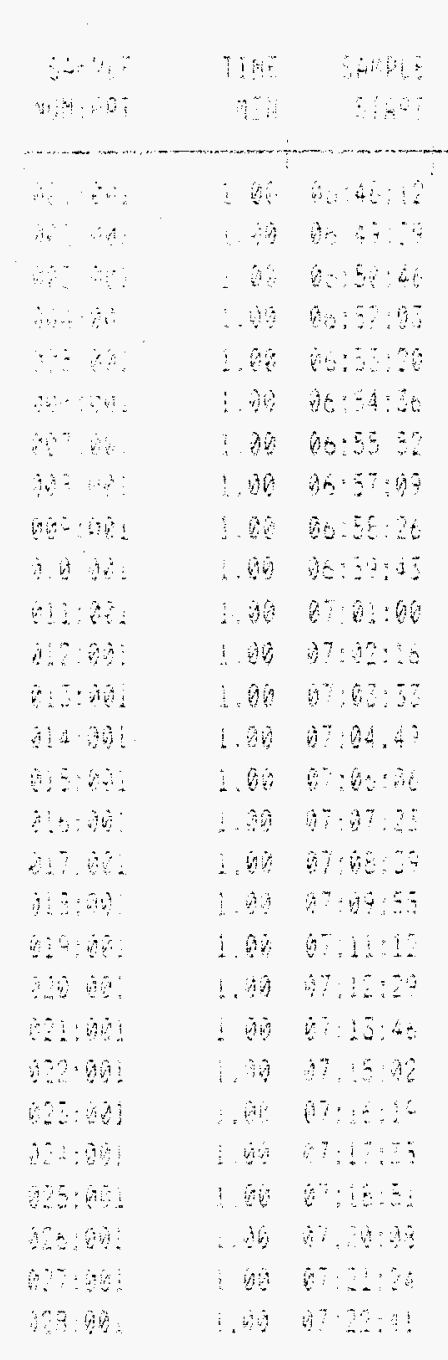

ges $\quad, 400$

Jf $7-16-05$ 


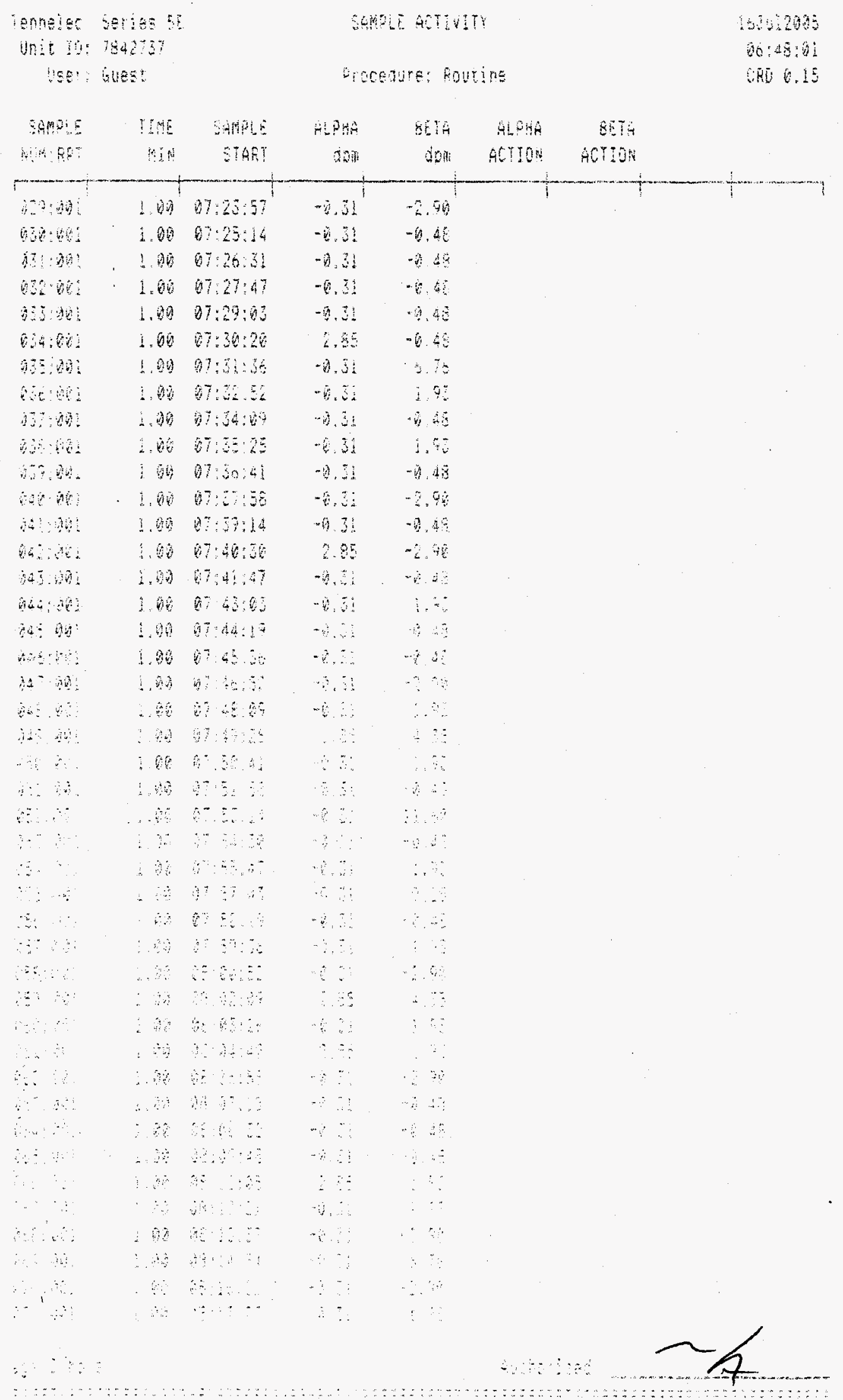


SOUVETOS-ERALSS-918 PAOE 28 of 34

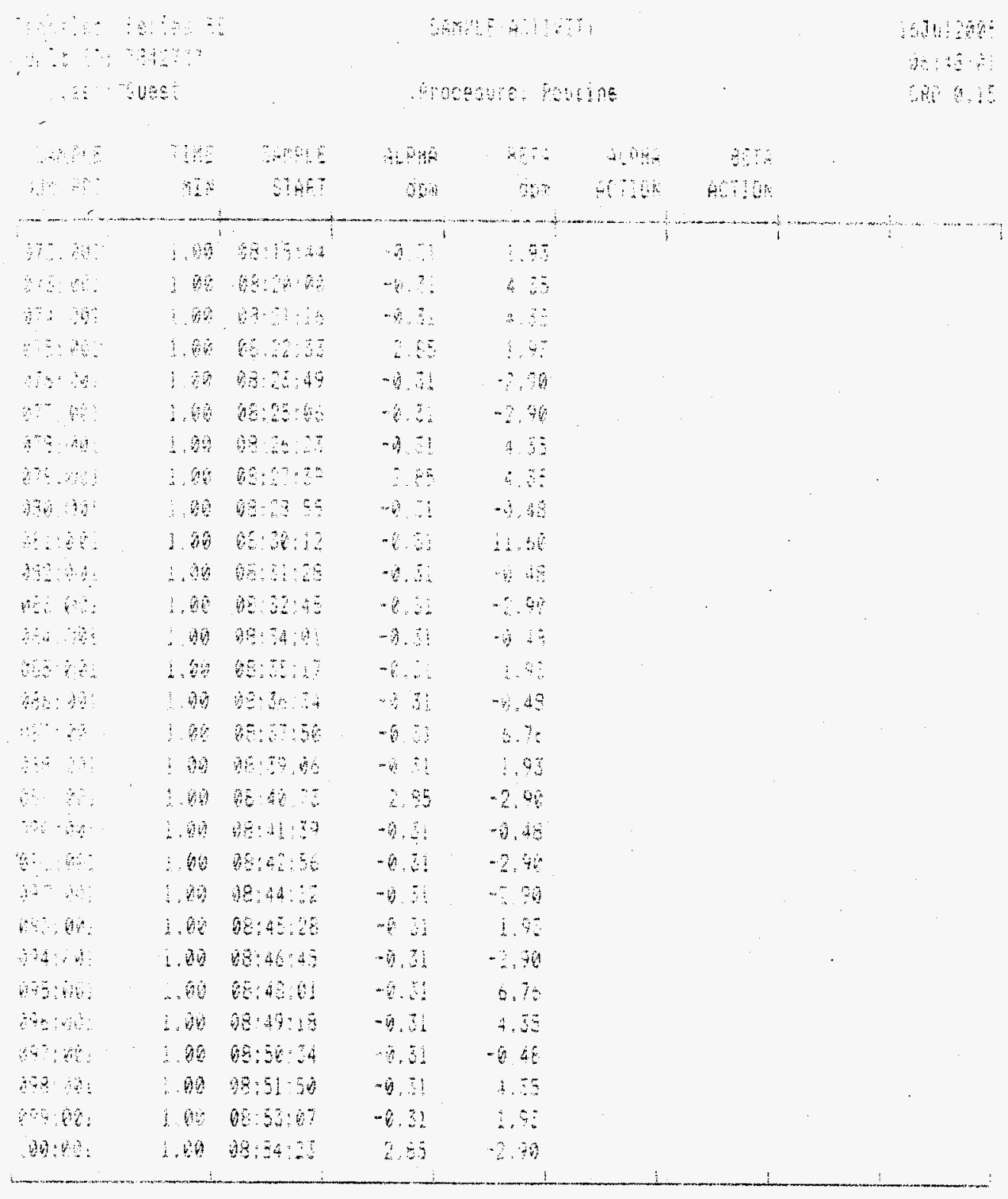

398 ₹ngne

Antias: 
SONUECOOS-ER-AZS- 718

Page 29 of 34

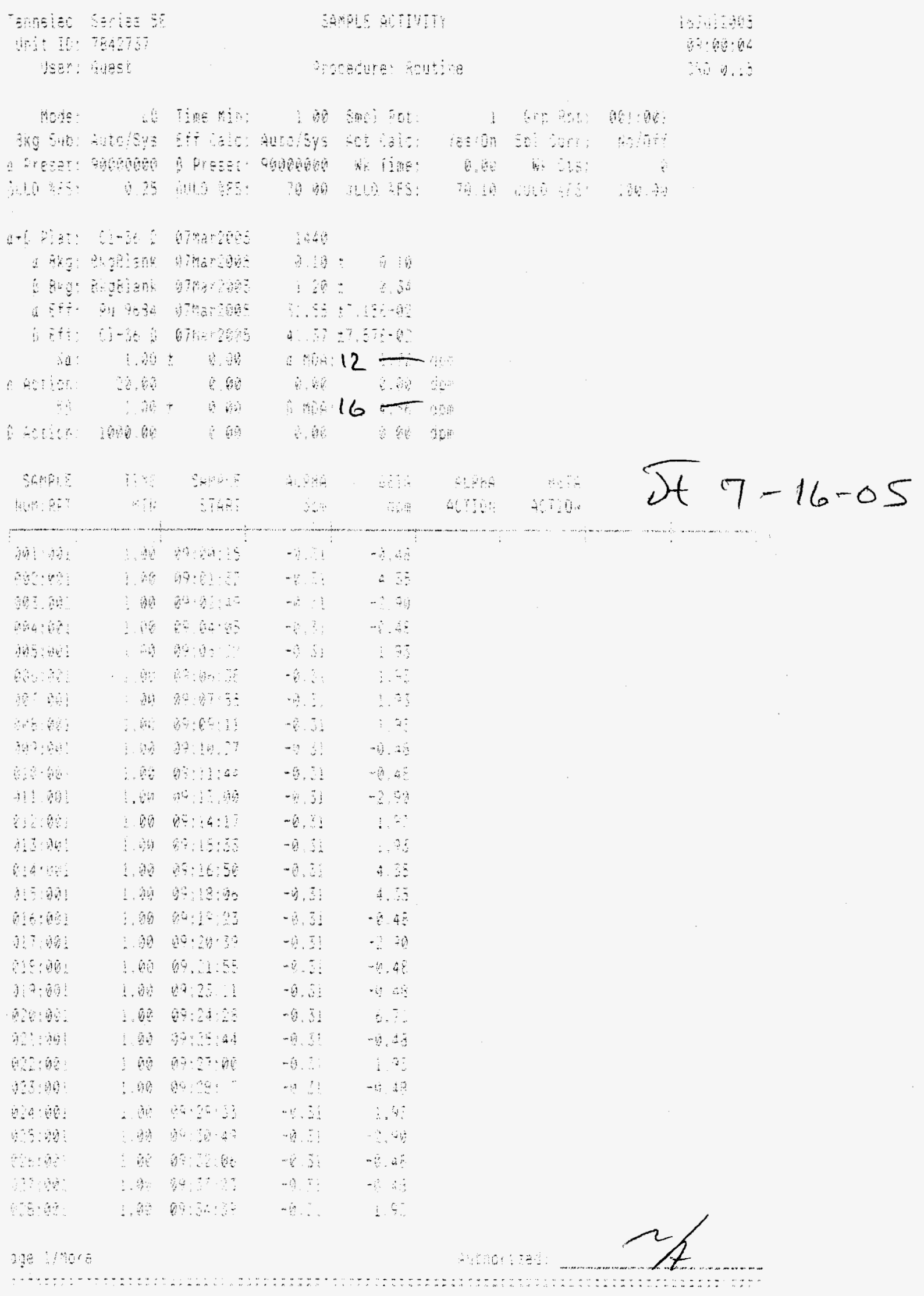




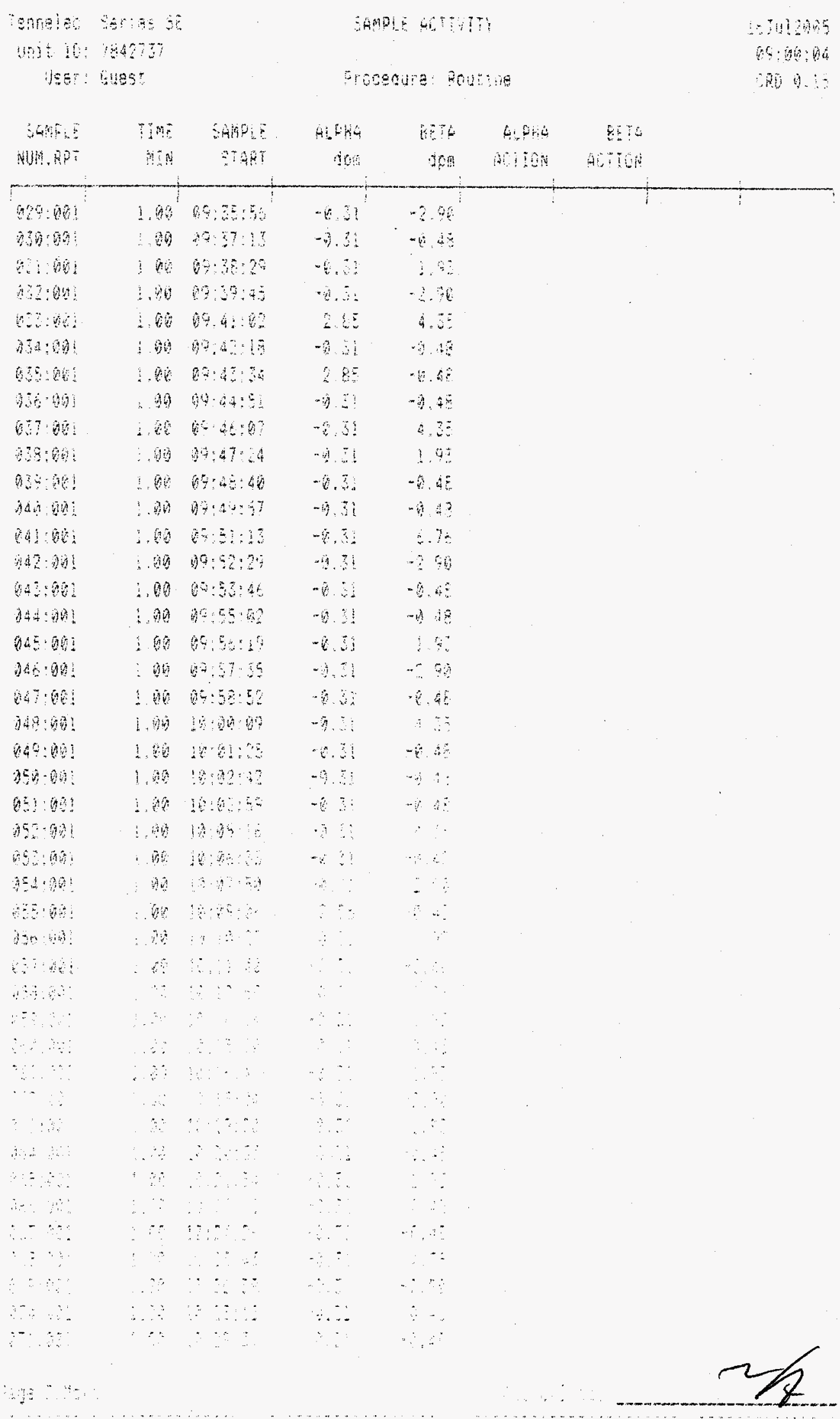


SOMUEY HOS-ER-A25-918 Page 31 of 34

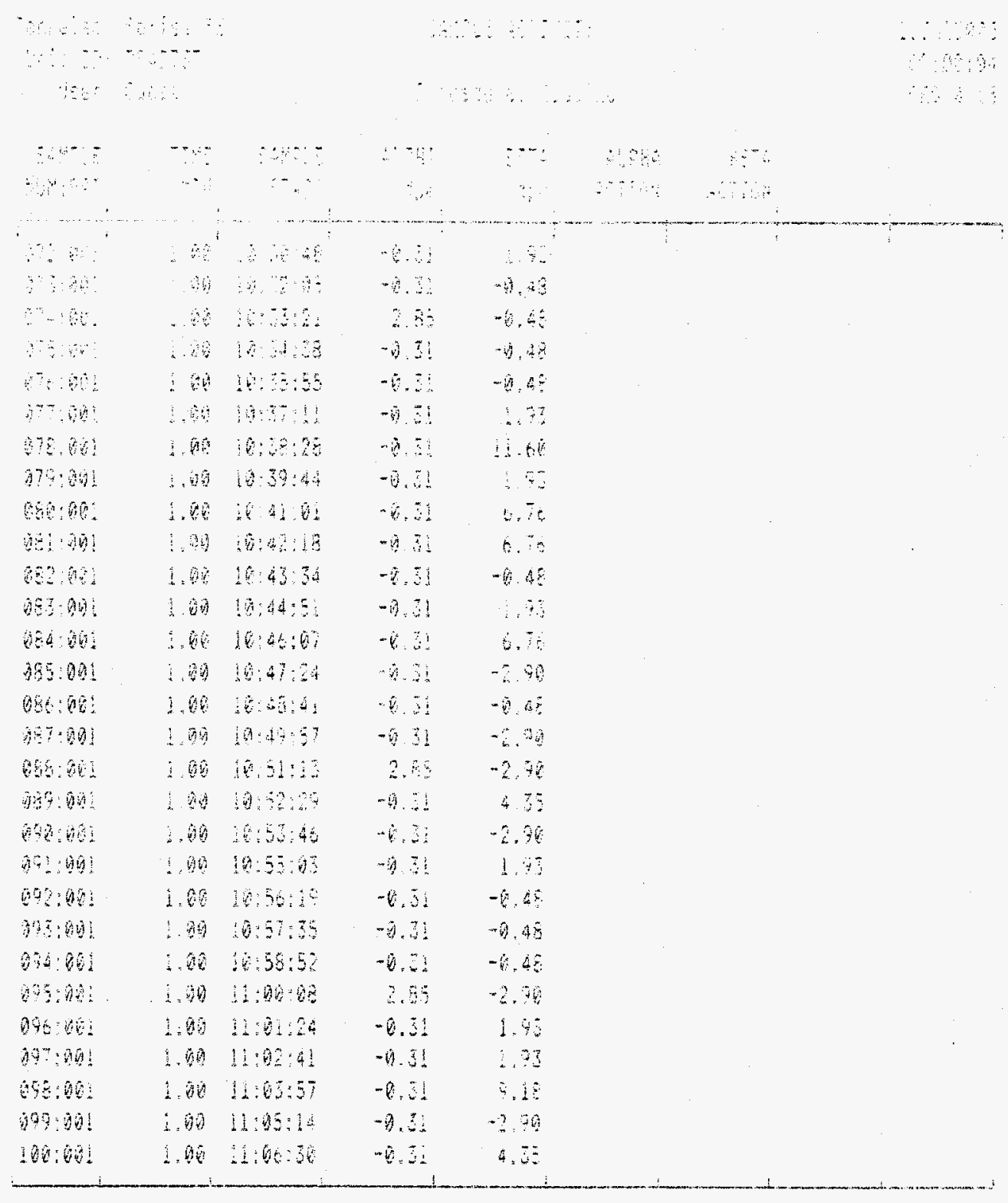

1gge i bone

41) 1206 
SONUEY FS-ER-A2S- T18

Thister series

Unit If: 768\%

SAMPE GrTITHY

procomaras putho mange 32 of 34

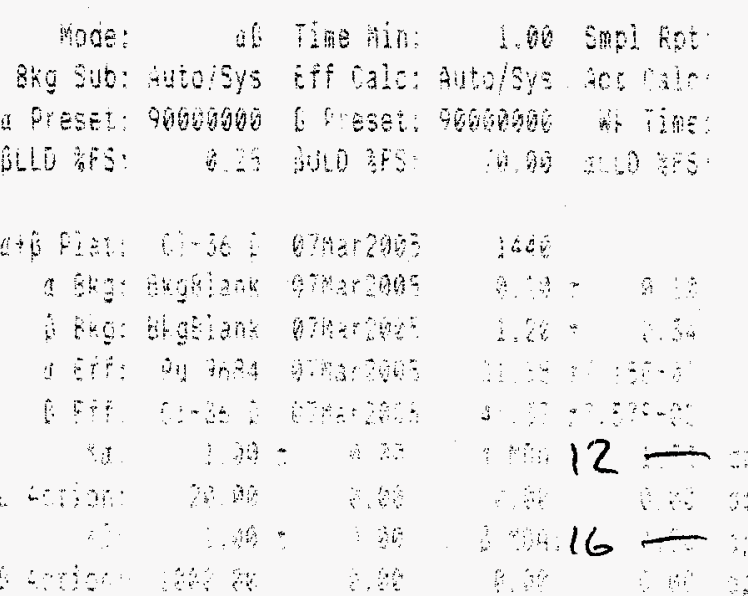

i 67 m 9

at at a

(a) 3

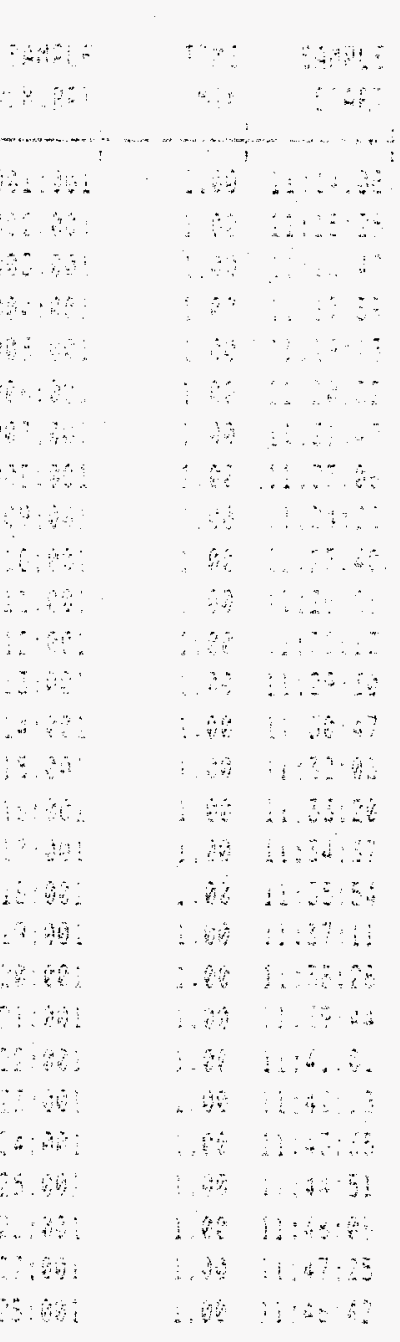

$\sqrt{t} 7-16-05$

$52+16=$

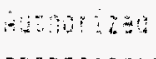




$$
\begin{aligned}
& \text { Sonver O5-EIR-125-718 } \\
& \text { PAGE }=3 \text { of } 34
\end{aligned}
$$

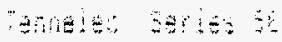

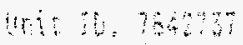

3ser: Gues:

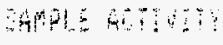

Poratire: Routine
1. 14645

11:5050

064.15

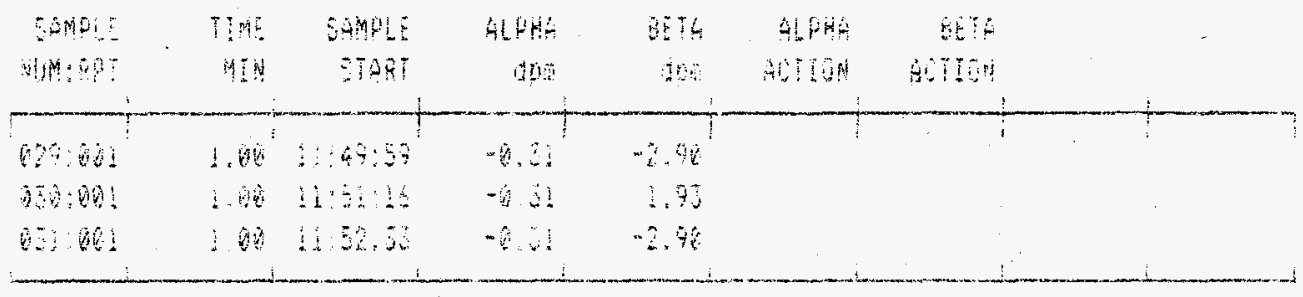

$20 \%$ - 1006

futhor ises

28 


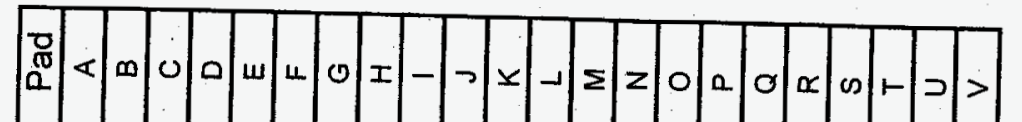

- - N N N ल ๓ ๓

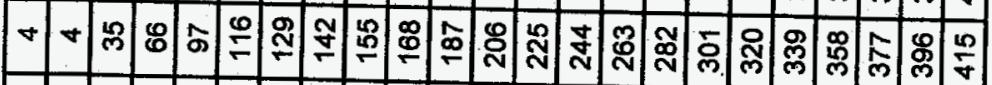
ம 1 :

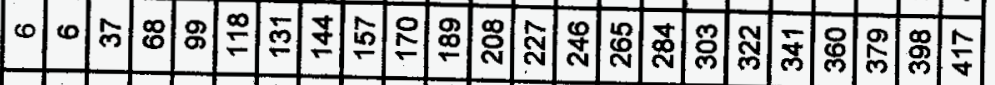

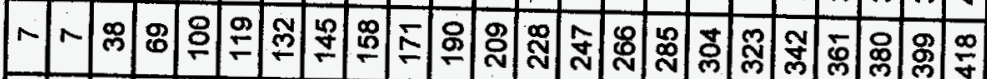

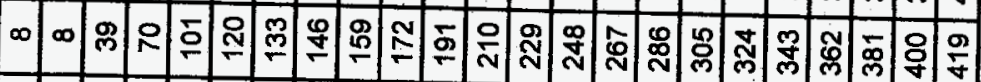

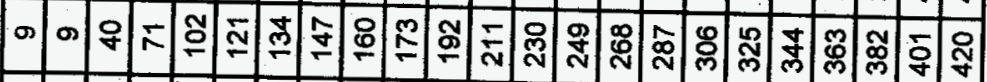

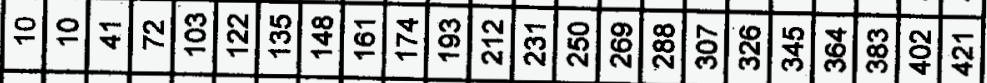

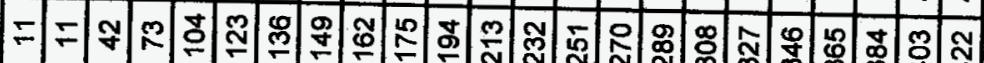
n

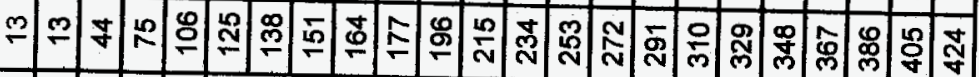
$\forall \div$ 年

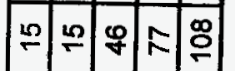
$\circ \div$ ₹ $\infty$ 웅 $\therefore=$ 원의

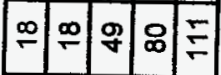
이유

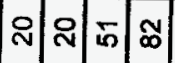

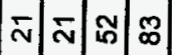
ๆ হ ஓ: ๘

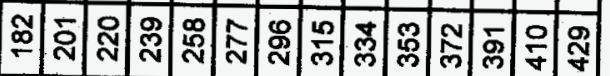
œ 
Closure Report - CAU 115

Section: Appendix D

Revision: 0

Date: March 2006

\section{APPENDIX D}

\section{WASTE DISPOSITION DOCUMENTATION}


Closure Report - CAU 115

Section: Appendix D

Revision: 0

Date: March 2006

THIS PAGE INTENTIONALLY LEFT BLANK 


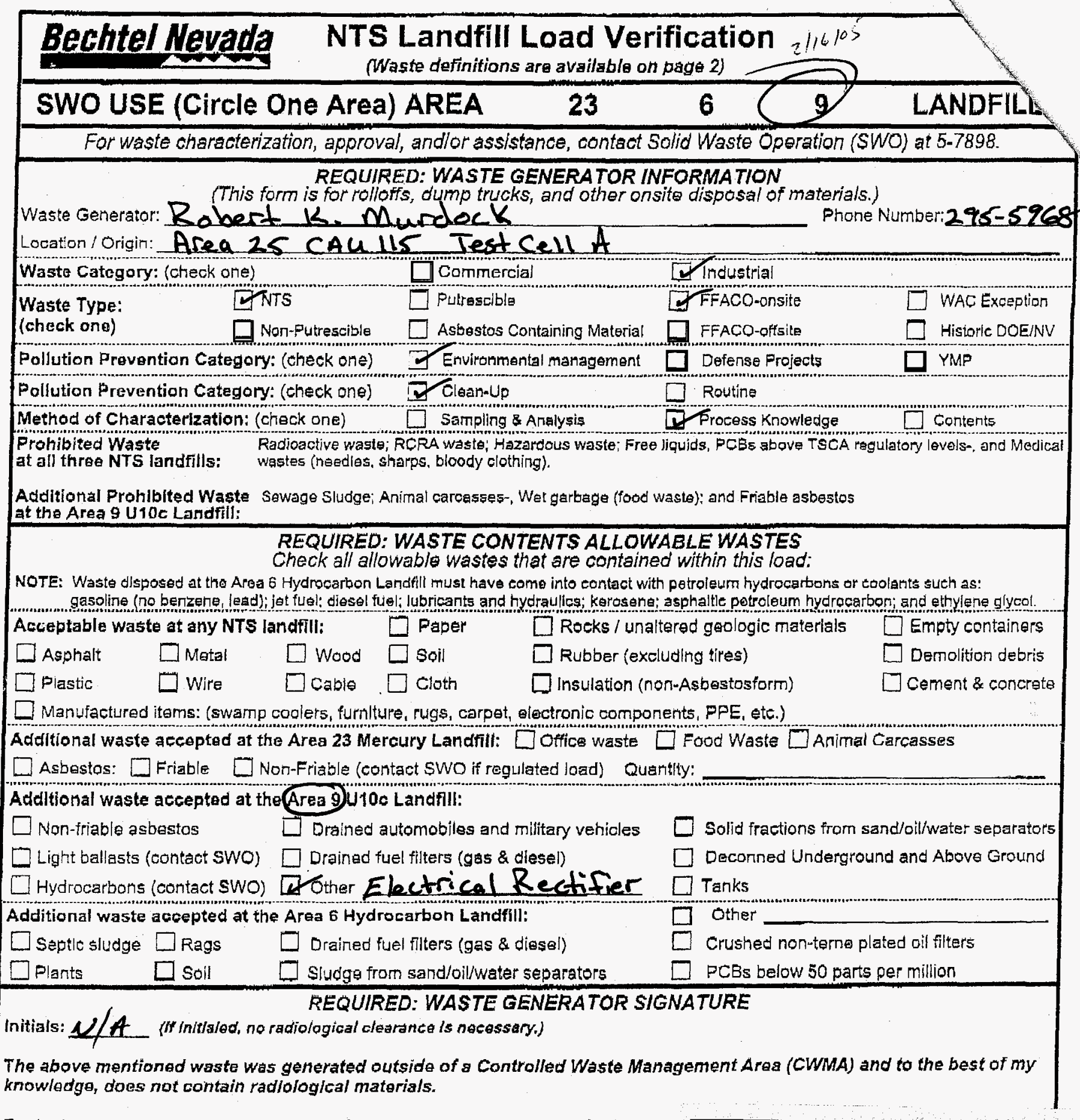

To the best of my knowledge, the waste described above contains only those mi site. I have veriffed this through the waste characterization method identified abc? prohlbited and allowable waste items.

Print Namg: Robert K. Murdock signature Pobut $Y$ Apuchl Date: $2-14.05$

Note: Food waste, office trash and/or anlmal careasses are considered not to contain Radiation Survey Release for Waste Disposal RCT Initials This containerload is free of external radioactive
contamination.

This container/load is exempt from survey due to process knowledge and origin

8 This container/load is free of radioactive - 2 contamination based on radioanalysis.

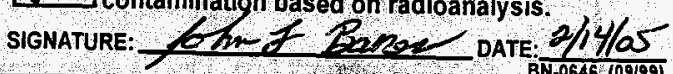
require a radiological clearance. SWO USE ONLY
Load Weight (net from scale gl estimate): 11 , 100 Signature of Certifie Ratantion Codo: ENV B.b

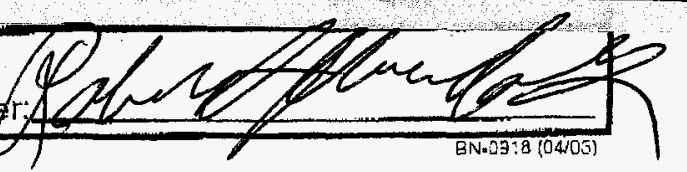
lEs-d $800 / 200 \mathrm{~d} \quad \varepsilon \varepsilon z-1$
1912562202

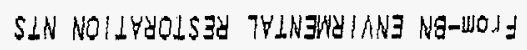
$00: 21 \quad 9002-6 !-403$ 
Somas 5 .

G82 0709A TRK

E104326

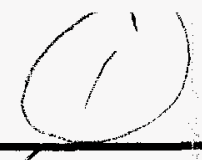

\section{Bechtel Nevada NTS Landfill Load Verification \\ (Waste definitions are available on page 2)}

$\begin{array}{lllll}\text { SWO USE (Circle One Area) AREA } & 23 & 6 & 9 & \text { LANDFILL }\end{array}$

For waste characterization, approval, and/or assistance, contact Solid Waste Operation (SWO) at 5-7898.

REQUIRED: WASTE GENERATOR INFORMATION

(This form is for rolloffs, dump trucks, and other onsite disposal of materials.)

Waste Generator: Be construction (nuike Kruzir) Phone Number: $5-7396$

Location / Origin: Area 25

Test cell $A$

(1)

$\square$ Commercial $\square$ Industrial

Waste Category: (check one)

Waste Type:

(check one)

$\square$ NTS $? / \mathrm{r} / \mathrm{u}$
$\square$ Non-Putrescible

$\square$ Putrescible

4 FFACO-onsite

$\square$ Asbestos Containing Material

FFACO-offsite

WAC Exception

Pollution Prevention Category: (check one)

Pollution Prevention Category: (check one)

7 Environmental management

Defense Projects

Routine

Method of Characterization: (check one) 7 Sampling \& Analysis

Process Knowledge

Contents

$\begin{array}{ll}\text { Prohibited Waste } & \text { Radioactive waste; RCRA waste; Hazardous } \\ \text { at all three NTS landfills: } & \text { wastes (needles, sharps, bloody clothing) }\end{array}$

Additional Prohibited Waste Sewage Sludge; Animal carcasses-, Wet garbage (food waste); and Friable asbestos

at the Area 9 U10c Landfill:

REQUIRED: WASTE CONTENTS ALLOWABLE WASTES
Check all allowable wastes that are contained within this load:

NOTE: Waste disposed at the Area 6 Hydrocarbon Landfill must have come into contact with petroleum hydrocarbons or coolants such as: gasoline (no benzere, lead); jet fuel; diesel fuel; lubricants and hydraulics; kerosene; asphaltic petroleum hydrocarbon; and ethylene glycol.

Acceptable waste at any NTS landfill: $\quad \square$ Paper $\square$ Rocks / unaltered geologic materials $\square$ Empty containers
$\square$ Asphalt
Thetal
7 Wood
$\square$ Soil
$\square$ Rubber (excluding tires)
$\exists$ Plastic $\quad$ Wire
$\square$ Cable $\square$ cloth
$\square$ insulation (non-Asbestosform)
$\exists$ Demoltion debris
Geement \& concrete

Manufactured items: (swamp coolers, furniture, rugs, carpet, electronic components, PPE, etc.)

Additional waste accepted at the Area 23 Mercury Landfill: $\square$ Office waste $\square$ Food Waste $\square$ Animal Carcasses

$\square$ Asbestos: $\square$ Friable $\square$ Non-Friable (contact SWO if regulated load) Quantity:

Additional waste accepted at the Area 9 U10c Landfill:
$\square$ Non-friable asbestos
Drained automobiles and military vehicles
Solid fractions from sand/oil/water separators
$\square$ Light ballasts (contact SWO) $\square$ Drained fuel filters (gas \& diesel)
Deconned Underground and Above Ground
Hydrocarbons (contact SWO)
Other
$\square$ Tanks
Additional waste accepted at the Area 6 Hydrocarbon Landfill:
$\square$ septic sludge $\square$ Rags
Drained fuel filters (gas \& diesel)
Other
$\square$ Plants
$\square$ Soil
Sludge from sand/oil/water separators
Crushed non-terne plated oil filters
PCBs below 50 parts per million

\section{REQUIRED: WASTE GENERATOR SIGNATURE}
Initials:
(If initialed, no radiological clearance is necessary.)

The above mentioned waste was generated outside of a Controlled knowledge, does not contain radiological materials.

To the best of my knowledge, the waste described above contains a site. I have verified this through the waste characterization method prohibited and allowable waste items.

Print Name: Robert $L$ Murdoek Radiation Survey Release for Waste Disposal
RCT Initials

$\square$ This containernoad is free of external radioactive this This container/load is exempt from survey due to 20

\section{process knowledge and origin. \\ D. contamination basedg fre of radioanalysis.}

SIGNATURE:

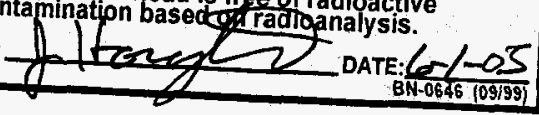

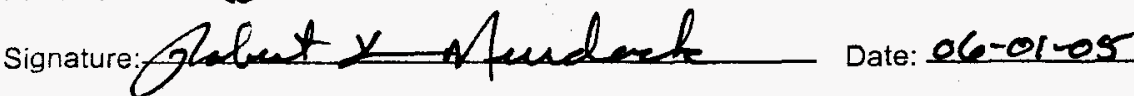

Note: Food waste, office trash and/or animal carcasses are considered not to contain added radioactivity, and therefore do not require a radiological clearance.

SWO USE ONLY

Load Weight (net from scale Qr estimate).) 27500

Signature of Certifier:
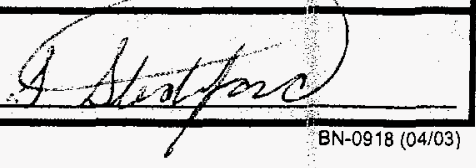
steve $C$.

\section{G82 0730A Trk \\ Bechtel Nevada NTS Landfill Load Verification \\ (Waste definitions are available on page 2)}

\begin{tabular}{lllll}
\hline SWO USE (Circle One Area) AREA & 23 & 6 & 9 & LANDFILL
\end{tabular}

For waste characterization, approval, and/or assistance, contact Solid Waste Operation (SWO) at 5-7898.

\section{REQUIRED: WASTE GENERATOR INFORMATION}

(This form is for rolloffs, dump trucks, and other onsite disposal of materials.)

Waste Generator: Bu construction (mile Vurgic)

Waste Category: (check one) $\square$ Commercial

Waste Type: $\quad \square$ TTS $/ 2 W 16-\square$ Putrescible

(check one)

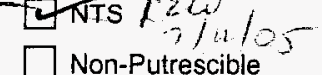

$\square$ Asbestos Cont

$F$ Industrial

Phone Number: $5-7396$

Pollution Prevention Category: (check one)

Pollution Prevention Category: (check one)

7 Environmental management

TFFACO-Onsite

WAC Exception

Method of Characterization: (check one)

9 Clean-Up

Prohibited Waste
at all three NTS land

7. Sampling \& Analysis

$\square$ FFACO-offsite

Radioactive waste; RCRA waste; Hazardous waste; Free liquids, PCBs above TSCA regulatory levels-, and Medical

at all three NTS landfills: wastes (needles, sharps, bloody clothing).

Additional Prohibited Waste Sewage Sludge; Animal carcasses-, Wet garbage (food waste); and Friable asbestos

at the Area 9 U10c Landfill:

REQUIRED: WASTE CONTENTS ALLOWABLE WASTES

Check all allowable wastes that are contained within this load:

NOTE: Waste disposed at the Area 6 Hydrocarbon Landfill must have come into contact with petroleum hydrocarbons or coolants such as:

gasoline (no benzene lead): jet fuel: diesel fuel: lubricants and hydraulics; kerosene; asphaltic petroleum hydrocarbon; and ethylene glycol.

Acceptable waste at any NTS landfill: $\quad \square$ Paper $\quad \square$ Rocks / unaltered geologic materials $\square$ Empty containers
$\square$ Asphalt
Metal
TWood
$\square$ Soil
$\square$ Rubber (excluding tires)
GPlastic
$\checkmark$ Wire
$\square$ Cable $\square$ Cloth
$\square$ Insulation (non-Asbestosform)
7 Demolition debris

$\square$ Manufactured items: (swamp coolers, furniture, rugs, carpet, electronic components, PPE, etc.)

Additional waste accepted at the Area 23 Mercury Landfill: $\square$ Office waste $\square$ Food Waste $\square$ Animal Carcasses

$\square$ Asbestos: $\square$ Friable $\square$ Non-Friable (contact SWO if regulated load) Quantity:

Additional waste accepted at the Area 9 U10c Landfill:

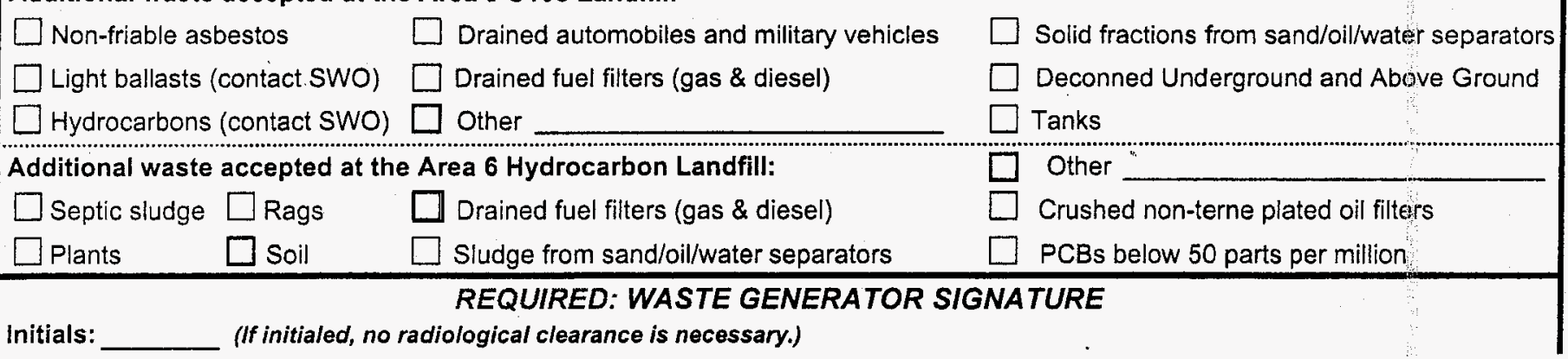

The above mentioned waste was generated outside of a Controlled Waste Mana knowledge, does not contain radiological materials.

To the best of my knowledge, the waste described above contains only those $m$ site. I have verified this through the waste characterization method identified ab prohibited and allowable waste items.

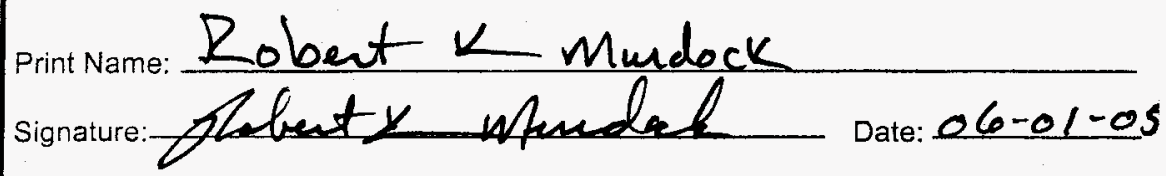

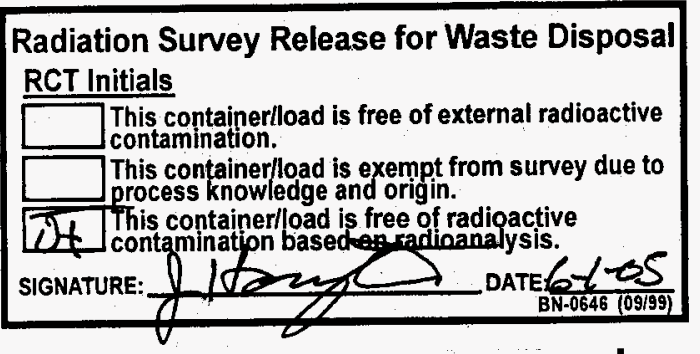

Note: Food waste, office trash and/or animal carcasses are considered not to contain added radioactivity, and therefore do not require a radiological clearance.

SWO USE ONLY

Load Weight (net from scale or estimate): 20,000 . Signature of Certifier 
Tobaes warren $M$.

G820731 A Trk

E 104817

$T R L$

\section{Bechtel Nevada NTS Landfill Load Verification}

(Waste definitions are available on page 2)

\section{SWO USE (Circle One Area) AREA \\ 23 \\ 6 \\ (9) \\ LANDFILL}

For waste characterization, approval, and/or assistance, contact Solid Waste Operation (SWO) at 5-7898.

\section{REQUIRED: WASTE GENERATOR INFORMATION}

(This form is for rolloffs, dymp trucks, and other onsite disposal of materials.)

Waste Generator: Bu Construction (mike Kruzic)

Location/Origin: Area 25 TesT cell A P.4i11

Waste Category: (check one)

Waste Type:

(check one)

GITS $\%, 1 a_{5} \square$ Putrescible

7 Industrial

$\square$ FFACO-onsite

Phone Number: $5-2376$

Pollution Prevention Category: (check one)

Pollution Prevention Category: (check one)

Method of Characterization: (check one)

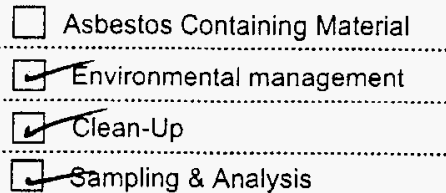

$\square$ FFACO-offsite

WAC Exception

Prohibited Waste

at all three NTS landfills:

G Sampling \& Analysis 7 Process Knowledge

$\square$ Contents

Additional Prohibited Waste Sewage Sludge; Animal carcasses-, Wet garbage (food waste); and Friable asbestos

at the Area 9 U10c Landfill:

\section{REQUIRED: WASTE CONTENTS ALLOWABLE WASTES}

Check all allowable wastes that are contained within this load:

NOTE: Waste disposed at the Area 6 Hydrocarbon Landfill must have come into contact with petroleum hydrocarbons or coolants such as: gasoline (no benzene, lead); jet fuel; diesel fuel; lubricants and hydraulics; kerosene; asphaltic petroleum hydrocarbon; and ethylene glycol.

Acceptable waste at any NTS landfill $\quad \square$ Paper $\quad \square$ Rocks / unaltered geologic materials $\square$ Empty containers
$\square$ Asphalt
$\checkmark$ Metal
Wood
$\square$ Soil
$\square$ Rubber (excluding tires)
$\checkmark$ Demolition debris
$\rightarrow$ Cemerit \& concrete

Plastic $\square$ Wire $\square$ Cable $\square$ Cioth $\square$ insulation (non-Asbestosform)
Manufactured items: (swamp coolers, furniture, rugs, carpet, electronic components, PPE, etc.)

Plastic $\square$ Wire $\square$ Cable $\square$ Cioth $\square$ insulation (non-Asbestosform)
Manufactured items: (swamp coolers, furniture, rugs, carpet, electronic components, PPE, etc.)

Plastic $\square$ Wire $\square$ Cable $\square$ Cioth $\square$ Insulation (non-Asbestosform)
Manufactured items: (swamp coolers, furniture, rugs, carpet, electronic components, PPE, etc.)

Additional waste accepted at the Area 23 Mercury Landfill: $\square$ office waste $\square$ Food Waste $\square$ Animal Carcasses

$\square$ Asbestos: $\square$ Friable $\square$ Non-Friable (contact SWO if regulated load) Quantity:

Additional waste accepted at the Area 9 U10c Landfill:
$\square$ Non-friable asbestos
$\square$ Drained automobiles and military vehicles
$\square$ Light ballasts (contact SWO)
$\square$ Drained fuel filters (gas \& diesel)
$\square$ Hydrocarbons (contact SWO)
Other
Additional waste accepted at the Area 6 Hydrocarbon Landfill:
$\square$ Septic sludge $\square$ Rags
$\square$ Plants
$\square$ Soil
$\square$ Drained fuel filters (gas \& diesel)
$\square$ Sludge from sand/oil/water separators
Initials: REQUIRED: WASTE GENERATOR SIGNATURE (If initialed, no radiological clearance is necessary.)
Solid fractions from sand/oil/water separators
$\square$ Deconned Underground and Above Ground Tanks
Other
$\square$ Crushed non-terne plated oil filters
PCBs below 50 parts per million

The above mentioned waste was generated outside of a Controlled Was knowledge, does not contain radiological materials.

To the best of my knowledge, the waste described above contains only site. I have verified this through the waste characterization method iden prohibited and allowable waste items.

Print Name: Robut $K$ Murdock signature:folvet $y$ Nfundech Date: 06-01-0s

\section{Radiation Survey Release for Waste Disposal} RCT Initials

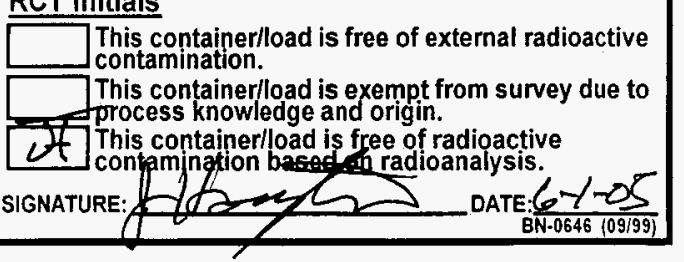

This container/load is free of external radioactive contamination.

Note: Food waste, office trash and/or animal carcasses are considered not to contain added radioactivity, and therefore do not require a radiological clearance.

SWO USE ONLY

Load Weight (net from scale or estimate): 
Tre Elo0035 Tames S

\section{Bechtel Nevada NTS Landfill Load Verification}

(Waste definitions are available on page 2)

\section{SWO USE (Circle One Area) AREA $23 \quad 6 \quad$ (9) LANDFILL}

For waste characterization, approval, and/or assistance, contact Solid Waste Operation (SWO) at 5-7898.

REQUIRED: WASTE GENERATOR INFORMATION

(This form is for rolloffs, dump trucks, and other onsite disposal of materials.)

Waste Generator: BNCOStruction (MiLe Kruzic)

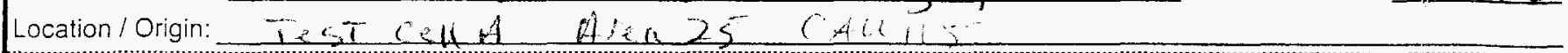

Waste Category: (check one) $\quad \square$ Commercial Thustrial

Waste Type: $\quad \square$ tNS $1=y, \quad \square$ Putrescible $\square$ FFACO-onsite $\square$ WACException

(check one) $\square$ Non-Putrescible; $\square$ Asbestos Containing Material $\square$ FFACO-offsite $\square$ Historic DOE/NV

Pollution Prevention Category: (check one) $\quad \square$ Environmental management $\square$ Defense Projects $\square$ YMP

Pollution Prevention Category: (check one) $\square$ Clean-Up $\square$ Routine

Method of Characterization: (check one) 7 Sampling \& Analysis $\quad 7$ Process Knowledge $\square$ Contents

Prohibited Waste $\quad$ Radioactive waste; RCRA waste; Hazardous waste; Free liquids, PCBs above TSCA regulatory levels-, and Medical at all three NTS landfills: wastes (needles, sharps, bloody clothing).

Additional Prohibited Waste Sewage Sludge; Animal carcasses-, Wet garbage (food waste); and Friable asbestos at the Area 9 U10c Landfill:

\section{REQUIRED: WASTE CONTENTS ALLOWABLE WASTES}

Check all allowable wastes that are contained within this load:

NOTE: Waste disposed at the Area 6 Hydrocarbon Landfill must have come into contact with petroleum hydrocarbons or coolants such as: gasoline (no benzene, lead); jet fuel; diesel fuel; lubricants and hydraulics; kerosene; asphaltic petroleum hydrocarbon; and ethylene glycol.

Acceptable waste at any NTS landfill: $\quad \square$ Paper $\quad \square$ Rocks / unaltered geologic materials $\square$ Empty containers
$\square$ Asphalt
Metal
7 Wood
$\square$ Soil
$\square$ Rubber (excluding tires)
Temolition debris

$\checkmark$ Plastic

7 Wire

$\square$ Cable $\square$ Cloth

$\square$ Insulation (non-Asbestosform)

Manufactured items: (swamp coolers, furniture, rugs, carpet, electronic components, PPE, etc.)

Additional waste accepted at the Area 23 Mercury Landfill: $\square$ Office waste $\square$ Food Waste $\square$ Animal Carcasses

$\square$ Asbestos: $\square$ Friable $\square$ Non-Friable (contact SWO if regulated load) Quantity:

Additional waste accepted at the Area 9 U10c Landfill:

\begin{tabular}{|c|c|c|}
\hline$\square$ Non-friable asbestos & $\square$ Drained automobiles and military vehicles & $\square$ Solid fractions from sand/oil/water separators \\
\hline$\square$ Light ballasts (contact SWO) & $\square$ Drained fuel filters (gas \& diesel) & $\square$ Deconned Underground and Above Ground \\
\hline$\square$ Hydrocarbons (contact SWO) & Other & $\square$ Tanks \\
\hline Additional waste accepted at th & Area 6 Hydrocarbon Landfill: & Other \\
\hline$\square$ septic sludge $\square$ Rags & $\square$ Drained fuel filters (gas \& diesel) & $\square$ Crushed non-terne plated oil filters \\
\hline$\square$ Plants $\quad \square$ soil & $\square$ Sludge from sand/oil/water separators & $\square$ PCBs below 50 parts per million \\
\hline
\end{tabular}

The above mentioned waste was generated outside of a Controlled Waste Mana knowledge, does not contain radiological materials.

To the best of my knowledge, the waste described above contains only those $m$ site. I have verified this through the waste characterization method identified ab prohibited and allowable waste items.

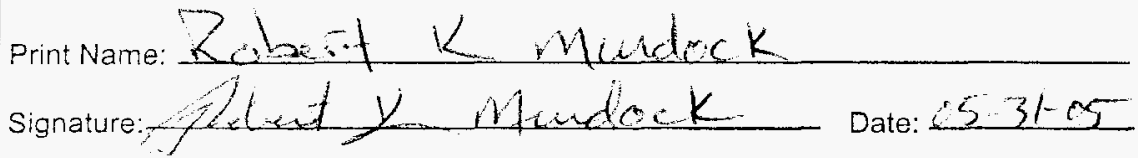

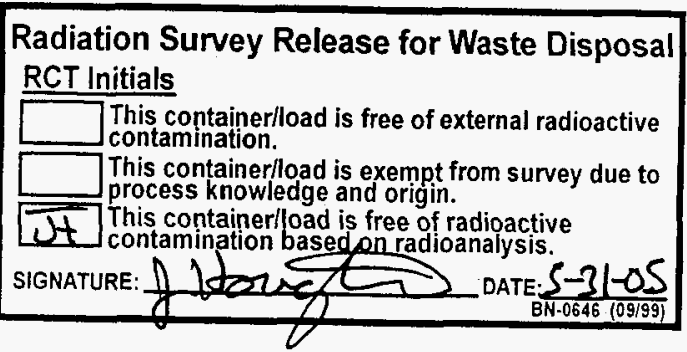

Note: Food waste, office trash and/or animal carcasses are considered not to contain added radioactivity, and therefore do not require a radiological clearance.

SWO USE ONLYY

Load Weight (net from scale, fr estimate): 16,2 


\section{Bechtel Nevada NTS Landfill Load Verification}

(Waste definitions are available on page 2)

\begin{tabular}{lllll}
\hline SWO USE (Circle One Area) AREA & 23 & 6 & 9 & LANDFILL
\end{tabular}

For waste characterization, approval, and/or assistance, contact Solid Waste Operation (SWO) at 5-7 98.

REQUIRED: WASTE GENERATOR INFORMATION

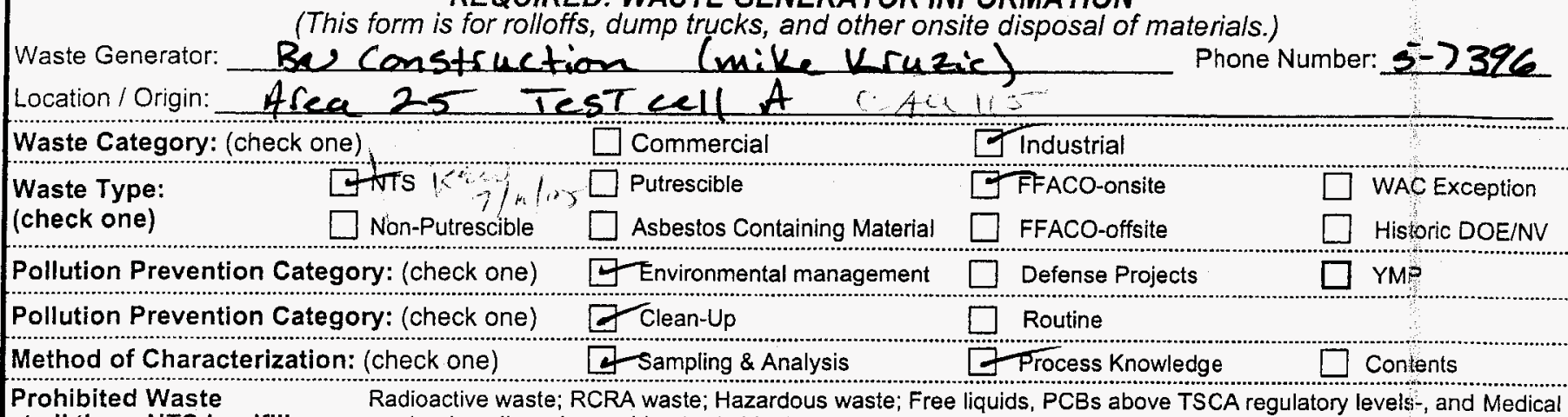
$\begin{array}{ll}\begin{array}{l}\text { Prohibited Waste } \\ \text { at all three NTS landfills: }\end{array} & \text { Radioactive waste; RCRA waste; Hazardo } \\ \text { wastes (needles, sharps, bloody clothing). }\end{array}$

Additional Prohibited Waste Sewage Sludge; Animal carcasses-, Wet garbage (food waste); and Friable asbestos at the Area 9 U10c Landfill:

\section{REQUIRED: WASTE CONTENTS ALLOWABLE WASTES}

Check all allowable wastes that are contained within this load:

NOTE: Waste disposed at the Area 6 Hydrocarbon Landfill must have come into contact with petroleum hydrocarbons or coolants such as: gasoline (no benzene, lead); jet fuel; diesel fuel; lubricants and hydraulics; kerosene; asphaltic petroleum hydrocarbon; and ethylene glycol.

Acceptable waste at any NTS landfill: $\square$ Paper $\square$ Rocks / unaltered geologic materials $\square$ Empty containers
$\square$ Asphalt
Metal
$\checkmark$ Wood
Soil
$\square$ Rubber (excluding tires)
$\exists$ Plastic
$\exists$ Wire
$\square$ Cable
Cloth
$\square$ Insulation (non-Asbestosform)
$\square$ Demolition debris Geement \& concrete

$\square$ Manufactured items: (swamp coolers, furniture, rugs, carpet, electronic components, PPE, etc.)

Additional waste accepted at the Area 23 Mercury Landfill: $\square$ Office waste $\square$ Food Waste $\square$ Animal Carcasses

Asbestos: $\square$ Friable $\square$ Non-Friable (contact SWO if regulated load) Quantity:

\section{Additional waste accepted at the Area 9 U10c Landfill:}
$\square$ Non-friable asbestos
$\square$ Drained automobiles and military vehicles
D] Light ballasts (contact SWO)
$\square$ Drained fuel filters (gas \& diesel)
$\square$ Hydrocarbons (contact SWO)
$\square$ Other

Additional waste accepted at the Area 6 Hydrocarbon Landfill:

\begin{tabular}{|c|c|c|}
\hline 7 septic sludge & $\square$ Rags & Drained fuel filters (gas \& diesel) \\
\hline Plants & Soil & Sludge from sand/oil/water separators \\
\hline
\end{tabular}

REQUIRED: WASTE GENERATOR SIGNATURE

Initials: (If initialed, no radiological clearance is necessary.)

The above mentioned waste was generated outside of a Controlled Waste Mana knowledge, does not contain radiological materials.

To the best of my knowledge, the waste described above contains only those $m$ site. I have verified this through the waste characterization method identified ab prohibited and allowable waste items.

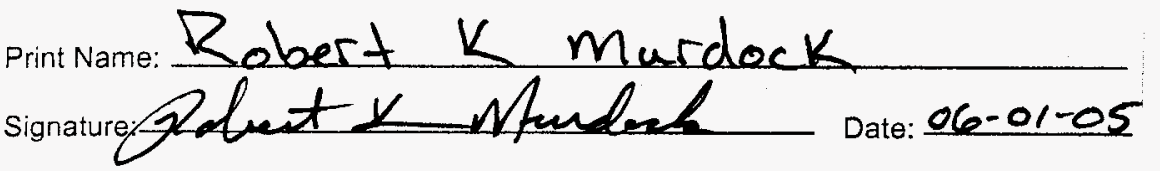

Note: Food waste, office trash and/or animal carcasses are considered not to contain added radioactivity, and therefore do not require a radiological clearance.

SWO USE ONLY

Load Weight (net from scale or estimate): 24,00

Solid fractions from sand/oil/water separators Deconned Underground and Above Ground

$\square$ Tanks

Other

Crushed non-terne plated oil filters

PCBs below 50 parts per million
Radiation Survey Release for Waste Disposal RCT Initials

$\square$ This container/load is free of external radioactive contamination.

This container/load is exempt from survey due to process knowledge and origin.

14 This containerload is frep of radioactive 12 conteminat on based endieanatysis.

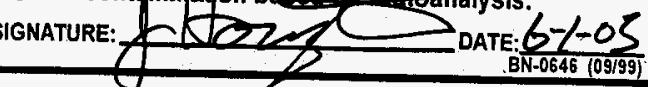




\begin{tabular}{lllll}
\hline SWO USE (Circle One Area) AREA & 23 & 6 & (9) LANDFILL
\end{tabular}

For waste characterization, approval, and/or assistance, contact Solid Waste Operation (SWO) at 5-7898.

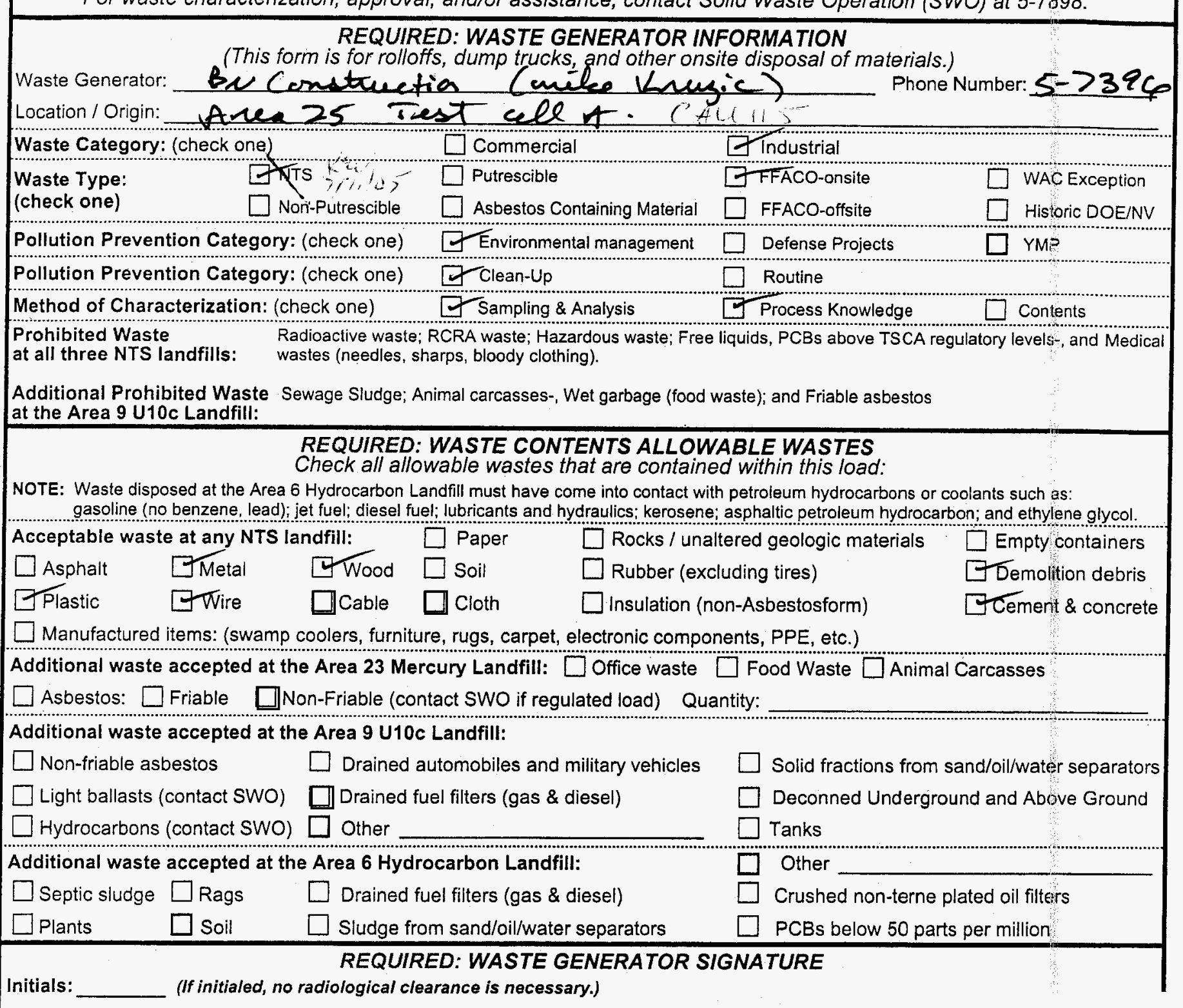

The above mentioned waste was generated outside of a Controlled Waste $\mathrm{Ma}$ knowledge, does not contain radiological materials.

To the best of my knowledge, the waste described above contains only those site. I have verified this through the waste characterization method identified prohibited and allowable waste items.

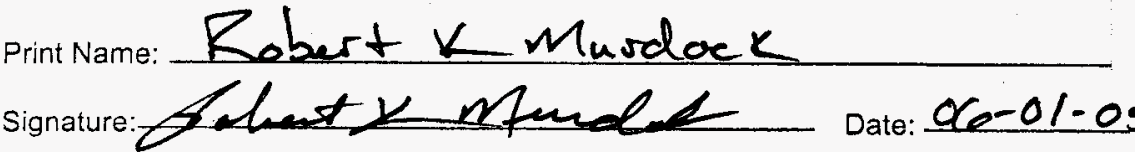

Radiation Survey Release for Waste Disposal RCT Initials

This containerlload is free of external radioactive contamination.

This container/load is exempt from survey due to

process knowledge and origin. ZR This container/load is free of radioactive SIGNATURE:

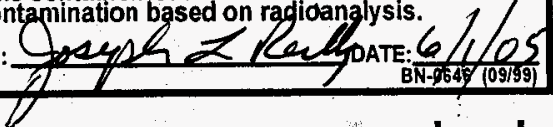

Note: Food waste, office trash and/or animal carcasses are considered not to contain added radioactivity, and therefore do not require a radiological clearance.

SWO USE ONLY

Load Weight (net from scale of stimate): 32,400 Signature of Certifier:
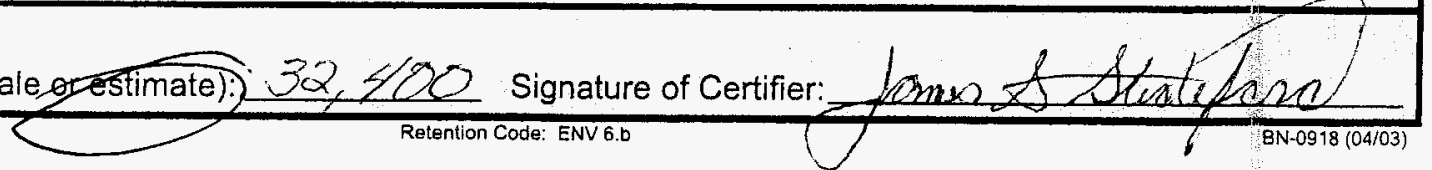


$\begin{array}{llllll}\text { SWO USE (Circle One Area) AREA } & 23 & 6 & 9 & \text { LANDFILL }\end{array}$

For waste characterization, approval, and/or assistance, contact Solid Waste Operation (SWO) at 5-7398.

REQUIRED: WASTE GENERATOR INFORMATION

(This form is for rolloffs, dump trucks, and other onsite disposal of materials.)

Waste Generator: Bu Construction (mul Kuygic)

Waste Category: (check one) $\quad \square$ Commercial $\quad \square$ Industrial

Waste Type: JTS k at $\square$ Putrescible

(check one) $\square$ Non-Putrescible $\square$ Asbestos Containing Material

Pollution Prevention Category: (check one) \& Environmental management

Pollution Prevention Category: (check one)

Method of Characterization: (check one)

7 Clean-Up

7 Sampling \& Analysis

7 FFACO-onsite $\square$ WAC Exception

$\square$ FFACO-offsite $\square$ Historic DOE/NV

Prohibited Waste

Radioactive waste; RCRA waste; Hazardous waste; Free liquids, PCBs above TSCA regulatory levels-, and Medical at all three NTS landfills: $\quad$ wastes (needles, sharps, bloody clothing).

Additional Prohibited Waste Sewage Sludge; Animal carcasses-, Wet garbage (food waste); and Friable asbestos at the Area 9 U10c Landfill:

\section{REQUIRED: WASTE CONTENTS ALLOWABLE WASTES}

Check all allowable wastes that are contained within this load:

NOTE: Waste disposed at the Area 6 Hydrocarbon Landfill must have come into contact with petroleum hydrocarbons or coolants such as: gasoline (no benzene, lead); jet fuel; diesel fuel; lubricants and hydraulics; kerosene; asphaltic petroleum hydrocarbon; and ethylene glycol.

Acceptable waste at any NTS landfill: $\square$ Paper $\square$ Rocks / unaltered geologic materials $\square$ Empty containers
$\square$ Asphalt
TMetal
7 Wood
$\square$ Soil
Cable $\square$ Cloth
$\square$ Rubber (excluding tires)
$\square$.nnsulation (non-Asbestosform)
$\exists$ Temolition debris
$\square$ Plastic $\square$ Wire

Manufactured items: (swamp coolers, furniture, rugs, carpet, electronic components, PPE, etc.)

Additional waste accepted at the Area 23 Mercury Landfill: $\square$ Office waste $\square$ Food Waste $\square$ Animal Carcasses

Asbestos: $\square$ Friable $\square$ Non-Friable (contact SWO if regulated load) Quantity:

Additional waste accepted at the Area 9 U10c Landfill:

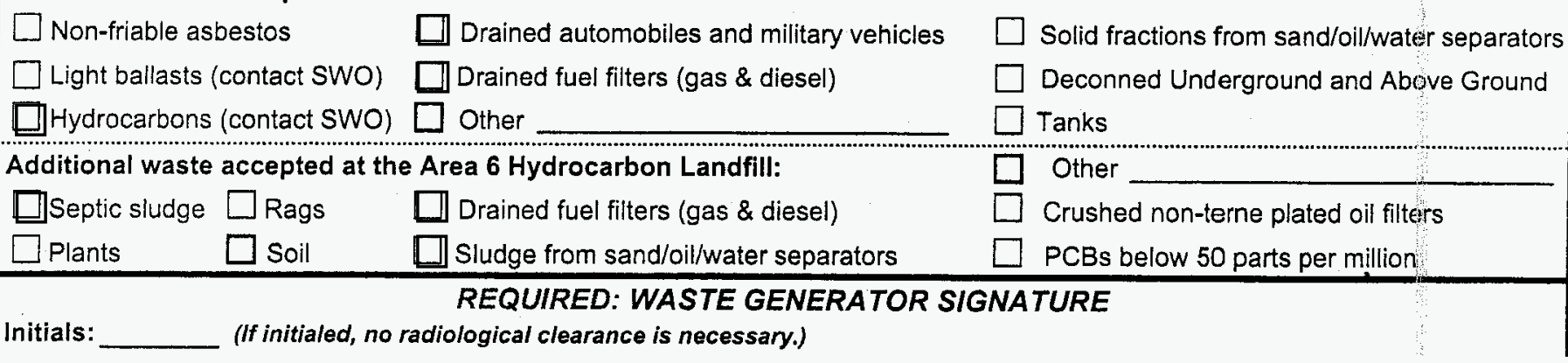

The above mentioned waste was generated outside of a Controlled Waste $M$ knowledge, does not contain radiological materials.

To the best of my knowledge, the waste described above contains only thos site. I have verified this through the waste characterization method identifiec prohibited and allowable waste items.

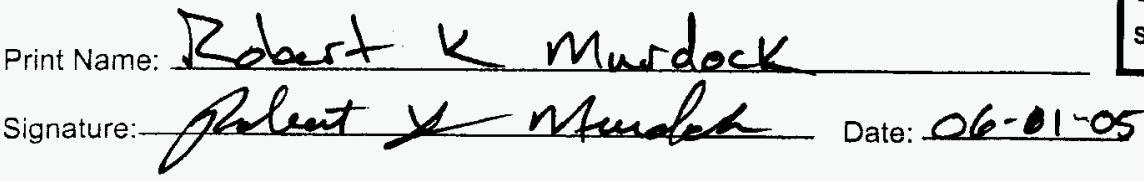

Radiation Survey Release for Waste Disposal RCT Initials

This containerlload is free of external radioactive contamination.

This container/load is exempt from survey due to process knowledge and origin.

It This container/load's yeerofradioactive

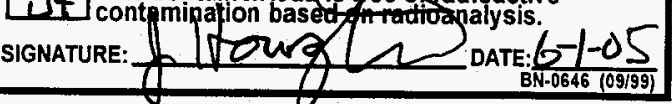

Note: Food waste, office trash and/or animal carcasses are considered not to contain added radioactivity, and therefore do not require a radiological clearance.

SWO USE ONLY

Load Weight (net from scale or estimate): 


\section{Bechtel Nevada NTS Landfill Load Verification}

(Waste definitions are available on page 2)

$\begin{array}{lllll}\text { SWO USE (Circle One Area) AREA } & 23 & 6 & 9 & \text { LANDFILL }\end{array}$

For waste characterization, approval, and/or assistance, contact Solid Waste Operation (SWO) at 5-7B9.8.

\section{REQUIRED: WASTE GENERATOR INFORMATION}

(This form is for rolloffs, dump trycks, and other onsite disposal of materials.)

Waste Generator: BN. Construction (Mile Kungic) Phone Number: $5-23 q 6$ Location/Origin: Alea 25 - Teut cell A.C.

Waste Type: $\quad \square$ NTS

(check one) $\square$ Non-Putrescible $\square$ Asbestos Containing Material

Pollution Prevention Category: (check one)

Pollution Prevention Category: (check one)

Method of Characterization: (check one)

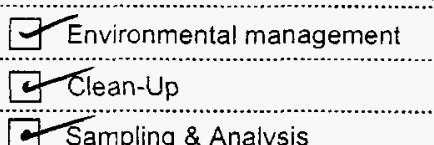

\begin{tabular}{l} 
Tndustrial \\
$\square$ FFACO-onsite \\
$\square$ FFACO-offsite \\
$\square$ Defense Projects \\
$\square$ Roc Historic DOEINV \\
$\square$ Routine \\
\hline Process Knowledge \\
\hline Contents
\end{tabular}

Prohibited Waste $\quad$ Radioactive waste; RCRA waste; Hazardous waste; Free liquids, PCBs above TSCA regulatory levels-, and Medical at all three NTS landfills: $\quad$ wastes (needles, sharps, bloody clothing).

Additional Prohibited Waste Sewage Sludge; Animal carcasses-, Wet garbage (food waste); and Friable asbestos at the Area 9 U10c Landfill:

\section{REQUIRED: WASTE CONTENTS ALLOWABLE WASTES}

Check all allowable wastes that are contained within this load:

NOTE: Waste disposed at the Area 6 Hydrocarbon Landfill must have come into contact with petroleum hydrocarbons or coolants such es: gasoline (no benzene, lead); jet fuel; diesel fuel; lubricants and hydraulics; kerosene; asphaltic petroleum hydrocarbon; and ethylene glycol.

Acceptable waste at any NTS landfill: $\quad \square$ Paper $\quad \square$ Rocks / unaltered geologic materials $\square$ Empty containers
$\square$ Asphalt
$\checkmark$ Metal
0 Wood
$\square$ Soil
$\square$ Rubber (excluding tires)
7 Plastic
7 Wire
$\square$ Cable $\square$ Cloth
$\square$ insulation (non-Asbestosform)
4 Demolition debris

Manufactured items: (swamp coolers, furniture, rugs, carpet, electronic components, PPE, etc.)

Additional waste accepted at the Area 23 Mercury Landfill: $\square$ Office waste $\square$ Food Waste $\square$ Animal Carcasses

$\square$ Asbestos: $\square$ Friable $\square$ Non-Friable (contact SWO if regulated load) Quantity:

\section{Additional waste accepted at the Area 9 U10c Landfill:}
$\square$ Non-friable asbestos
Drained automobiles and military vehicles
$\square$ Light ballasts (contact SWO)
$\square$ Drained fuel filters (gas \& diesel)
Hydrocarbons (contact SWO)
Other

Additional waste accepted at the Area 6 Hydrocarbon Landfill:
$\square$ septic sludge $\square$ Rags
$\square$ Plants
$\square$ Soil

$\square$ Drained fuel filters (gas \& diesel)

Sludge from sand/oil/water separators

\section{REQUIRED: WASTE GENERATOR SIGNATURE}

Initials: (If initialed, no radiological clearance is necessary.)

The above mentioned waste was generated outside of a Controlled Waste knowledge, does not contain radiological materials.

To the best of my knowledge, the waste described above contains only thi site. I have verified this through the waste characterization method identifi prohibited and allowable waste items.

Print Name: Rdaret $K$ murdoek

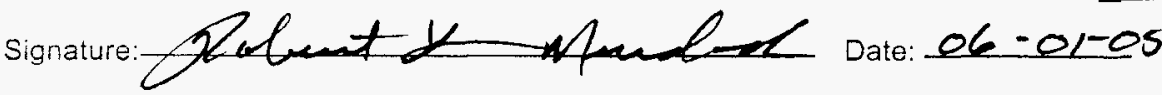

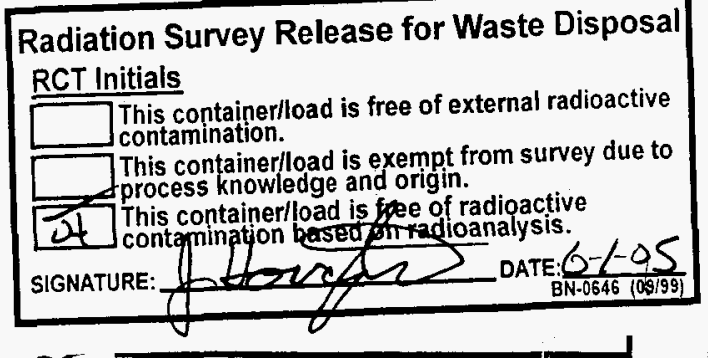

Note: Food waste, office trash and/or animal carcasses are considered not to contain added radioactivity, and therefore do not require a radiological clearance.

SWO USE ONLY

Load Weight (net from scale ofesttmate):, 500
Solid fractions from sand/oil/water separators

Deconned Underground and Above Ground Tanks

Other

$\square$ Crushed non-terne plated oil filters

$\square$ PCBs below 50 parts per million 
(Waste definitions are available on page 2)

$\begin{array}{llll}\text { SWO USE (Circle One Area) AREA } & 23 & 6 & 9\end{array}$ LANDFILL

For waste characterization, approval, and/or assistance, contact Solid Waste Operation (SWO) at 5 -7898.

$$
\text { REQUIRED: WASTE GENERATOR INFORMATION }
$$

(This form is for rolloffs, dump trucks, and other onsite disposal of materials.)

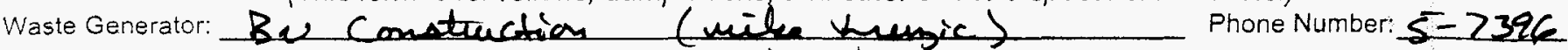
Location / Origin: Area 25 Testcell Waste Category: (check one)

Waste Type:

7 NTS

(check one)

\section{$\square$ Commercial}

$\square$ Putrescible

Pollution Prevention Category: (check one)

Pollution Prevention Category: (check one)

Method of Characterization: (check one)

$\square$ Asbestos Containing Material

4 Environmental management

4 Clean-Up

7 Sampling \& Analysis

Prohibited Waste

at all three NTS landfills:

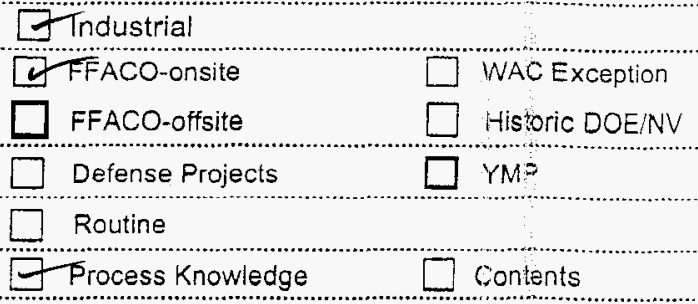

Radioactive waste; RCRA waste; Hazardous waste; Free liquids, PCBs above TSCA regulatory levelsis, and Medical wastes (needles, sharps, bloody clothing).

Additional Prohibited Waste Sewage Sludge; Animal carcasses-, Wet garbage (food waste); and Friable asbestos at the Area 9 U10c Landfill:

\section{REQUIRED: WASTE CONTENTS ALLOWABLE WASTES}

Check all allowable wastes that are contained within this load:

NOTE: Waste disposed at the Area 6 Hydrocarbon Landfill must have come into contact with petroleum hydrocarbons or coolants such as: gasoline (no benzene, lead); jet fuel; diesel fuel; fubricants and hydraulics; kerosene; asphaltic petroleum hydrocarbon; and $€$ hylene glycol.

Acceptable waste at any NTS landfill $\quad \square$ Paper $\quad \square$ Rocks / unaltered geologic materials

$\square$ Asphalt $\quad$ Metal $\square$ Wood $\square$ soil $\square$ Rubber (excluding tires)

Flastic

7 Wire

$\square$ Cable

Cloth

$\square$ insulation (non-Asbestosform)

Empty containers

Demolition debris $\exists$ Centert \& concrete

Manufactured items: (swamp coolers, furniture, rugs, carpet, electronic components, PPE, etc.)

Additional waste accepted at the Area 23 Mercury Landfill: $\square$ Office waste $\square$ Food Waste $\square$ Animal Carcasses

$\square$ Asbestos: $\square$ Friable $\square$ Non-Friable (contact SWO if regulated load) Quantity:

Additional waste accepted at the Area 9 U10c Landfill:

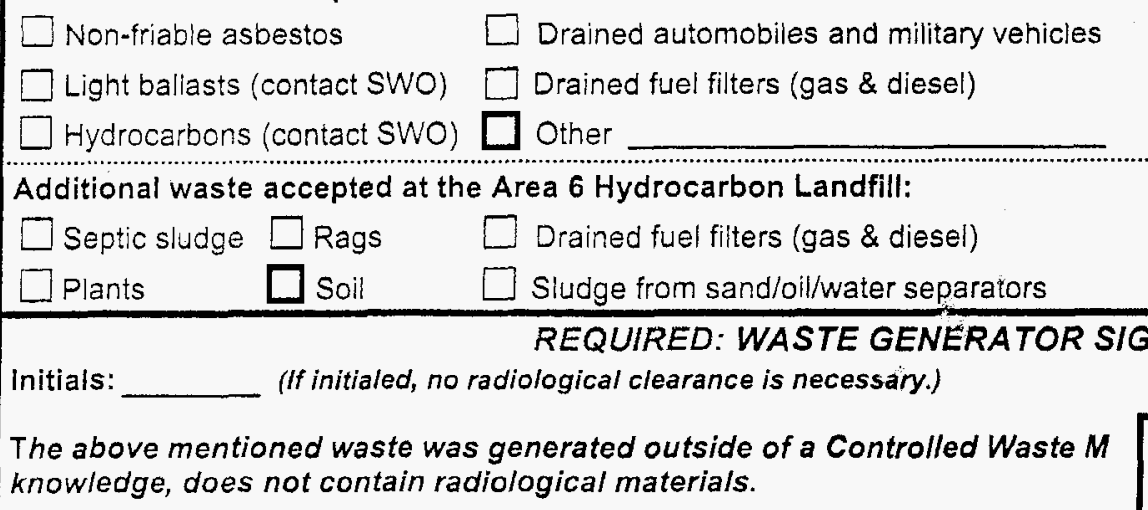

To the best of my knowledge, the waste described above contains only thos site. I have verified this through the waste characterization method identifier prohibited and allowable waste items.

Print Name: Robest 2 murdock

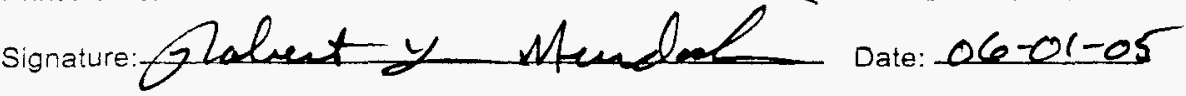

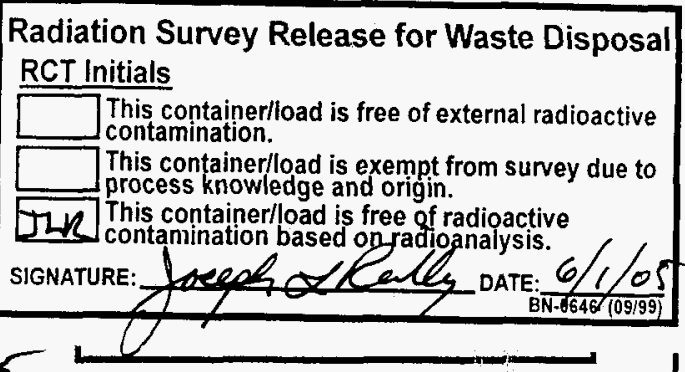

Note: Food waste, office trash and/or animal carcasses are considered not to contain added radioactivity, and therefore do not reguire a radiological clearance.

\section{SWO USE ONLY}

Load Weight (net from scale or estimatè

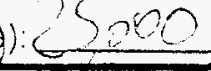

Signature of Certifier:

Solid fractions from sand/oil/water separators

Deconned Underground and Above Ground

Tanks

Other

Crushed non-terne plated oil ilters

PCBs below 50 parts per million

ATURE

Retention Code: ENv 6.6 


\section{Bechtel Nevada NTS Landfill Load Verification \\ (Waste definitions are available on page 2)

\begin{tabular}{lllll}
\hline SWO USE (Circle One Area) AREA & 23 & 6 & 9 & LANDFILL
\end{tabular}

For waste characterization, approval, and/or assistance, contact Solid Waste Operation (SWO) at 5-7398.

REQUIRED: WASTE GENERATOR INFORMATION

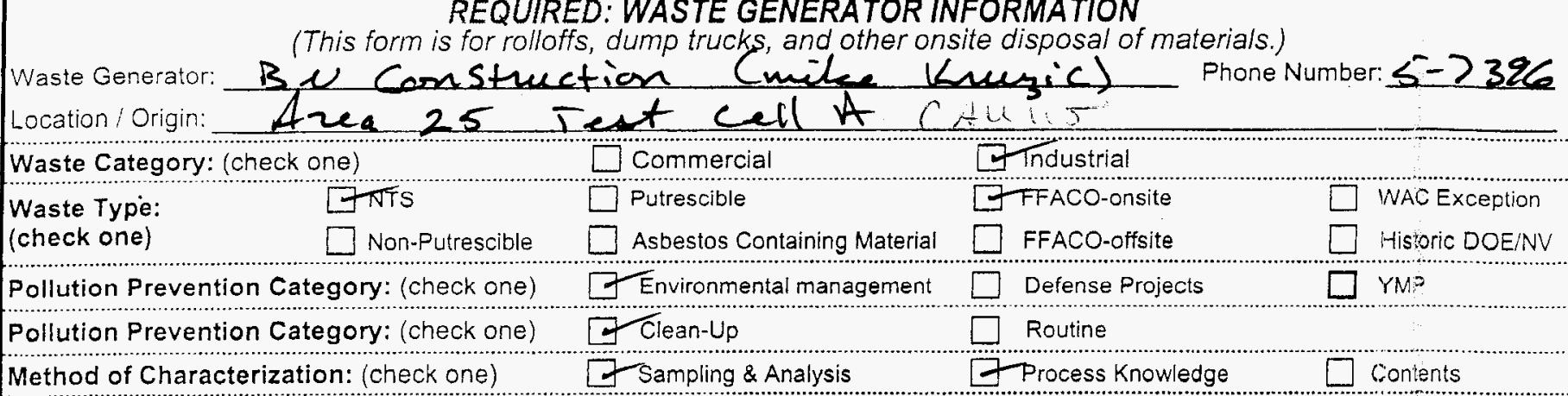

Prohibited Waste $\quad$ Radioactive waste; RCRA waste; Hazardous waste; Free liquids, PCBs above TSCA regulatory levelsi, and Medical at all three NTS landfills: wastes (needles, sharps, bloody clothing).

Additional Prohibited Waste Sewage Sludge; Animal carcasses-, Wet garbage (food waste); and Friable asbestos at the Area 9 U10c Landfill:

REQUIRED: WASTE CONTENTS ALLOWABLE WASTES
Check all allowable wastes that are contained within this load:

NOTE: Waste disposed at the Area 6 Hydrocarbon Landfill must have come into contact with petroleum hydrocarbons or coolants such as: gasoline (no benzene, lead); jet fuel; diesel fuel: lubricants and hydraulics; kerosene; asphaltic petroleum hydrocarbon; and ethylene giycol.

\begin{tabular}{|c|c|c|c|c|c|}
\hline Acceptable & at any & : & $\square$ Paper & $\square$ Rocks / unaltered geologic materials & $\square$ Empty containers \\
\hline$\square$ Asphalt & Metal & Wood & Soil & $\square$ Rubber (excluding tires) & Dermoltion debris \\
\hline PPlastic & 7 Wire & Cable & Cloth & $\square$ Insulation (non-Asbestosform) & TCenterit \& concrete \\
\hline
\end{tabular}

Plastic

7 Wire

$\square$ Cable $\square$ Cloth

Insulation (non-Asbestosform)

$\square$ Manufactured items: (swamp coolers, furniture, rugs, carpet, electronic components, PPE, etc.)

Additional waste accepted at the Area 23 Mercury Landfill: $\square$ Office waste $\square$ Food Waste $\square$ Animal Carcasses

$\square$ Asbestos: $\square$ Friable $\square$ Non-Friable (contact SWO if regulated load) Quantity:

Additional waste accepted at the Area 9 U10c Landfill:

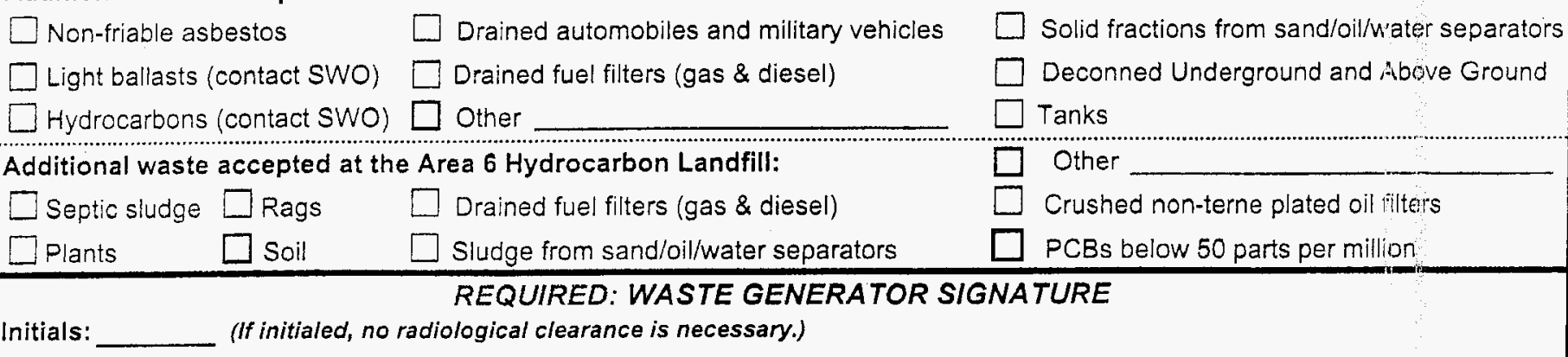

The above mentioned waste was generated outside of a Controlled Waste Mana knowledge, does not contain radiological materials.

To the best of my knowledge, the waste described above contains only those $m$ site. I have verified this through the waste characterization method identified at prohibited and allowable waste items.

Print Name: $\mathrm{Rabert} \mathrm{K}$ Murdock

Signature:flulust N4unclen Date:06-01-0s

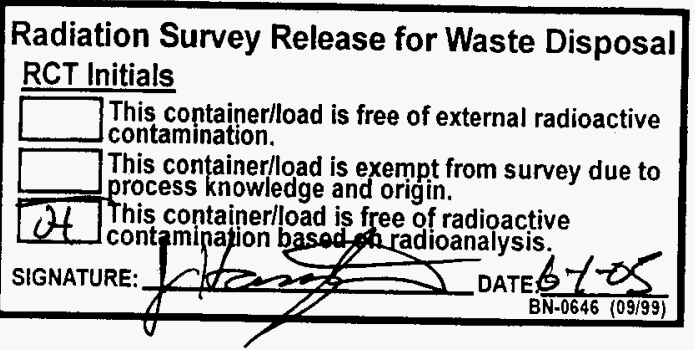

Note: Food waste, office trash and/or animal carcasses are considered not to contain added radioactivity, and therefore do not require a radiological clearance.

SWO USE ONLY 


\begin{tabular}{lllll}
\hline SWO USE (Circle One Area) AREA & 23 & 6 & 9 & LANDFILL
\end{tabular}

For waste characterization, approval, and/or assistance, contact Solid Waste Operation (SWO) at 5-7898.

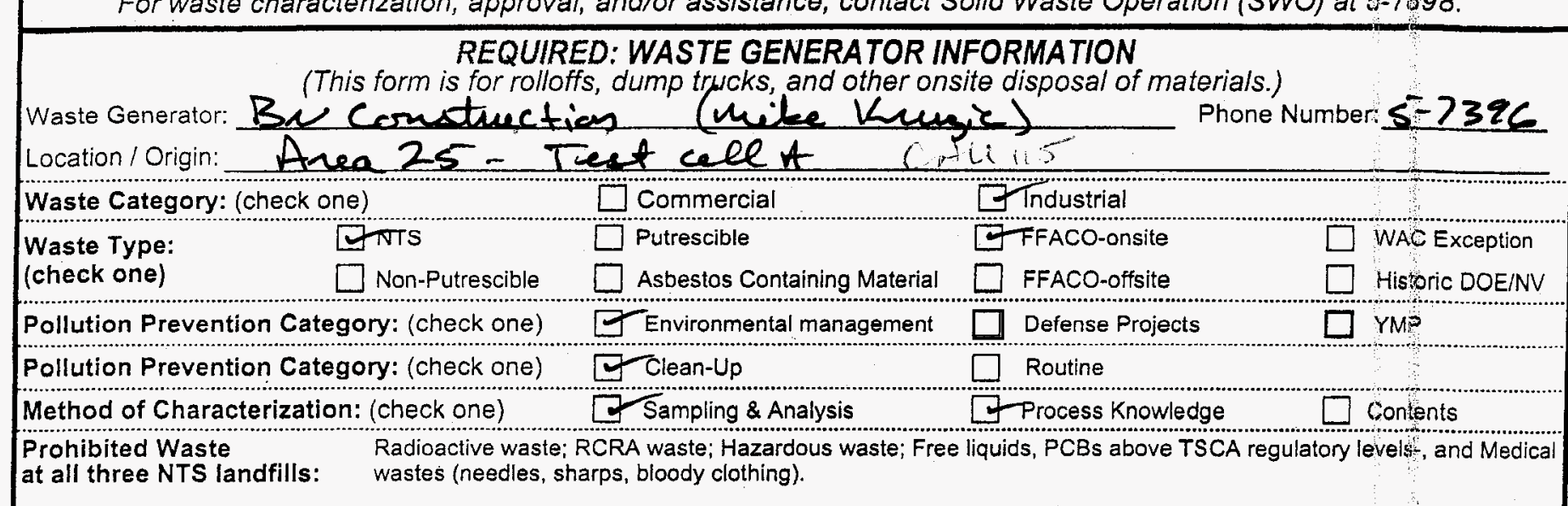

Additional Prohibited Waste Sewage Sludge; Animal carcasses-, Wet garbage (food waste); and Friable asbestos at the Area 9 U10c Landfill:

\section{REQUIRED: WASTE CONTENTS ALLOWABLE WASTES}

Check all allowable wastes that are contained within this load:

NOTE: Waste disposed at the Area 6 Hydrocarbon Landfill must have come into contact with petroleum hydrocarbons or coolants suich as: gasoline (no benzene, lead) jet fuel; diesel fuel; lubricants and hydraulics; kerosene; asphaltic petroleum hydrocarbon; and ethylene glycol.
Acceptable waste at any NTS landfill:
$\square$ Paper
$\square$ Rocks / unaltered geologic materiais
$\square$ Empty containers
$\square$ Asphalt
$G$ Metal
Whood
$\square$ Soil
7 Wire
$\square$ Cable $\square$ Cloth
$\square$ Rubber (excluding tires)
$\square$ Insulation (non-Asbestosform)
Teemolition debris
$\checkmark$ Flastic

Manufactured items: (swamp coolers, furniture, rugs, carpet, electronic components, PPE, etc.)

Additional waste accepted at the Area 23 Mercury Landfill: $\square$ Office waste $\square$ Food Waste $\square$ Animal Carcasses

$\square$ Asbestos: $\square$ Friable $\square$ Non-Friable (contact SWO if regulated load) Quantity:

Additional waste accepted at the Area 9 U10c Landfill:
$\square$ Non-friable asbestos
$\square$ Drained automobiles and military vehicles
$\square$ Light baliasts (contact SWO)
$\square$ Drained fuel filters (gas \& diesel)
Hydrocarbons (contact SWO)

Additional waste accepted at the Area 6 Hydrocarbon Landfill:

$\square$ Septic sludge $\square$ Rags

$\square$ Plants

$\square$ Soil $\square$ Drained fuel filters (gas \& diesel)

$\square$ Sludge from sand/oil/water separators
Solid fractions from sand/oil/water separators

Deconned Underground and Above Ground Tanks

Other

Crushed non-terne plated oil filters PCBs below 50 parts per million

\section{REQUIRED: WASTE GENERATOR SIGNATURE}

Initials: (If initialed, no radiological clearance is necessary.)

The above mentioned waste was generated outside of a Controlled W knowledge, does not contain radiological materials.

To the best of my knowledge, the waste described above contains onl. site. I have verified this through the waste characterization method ide prohibited and allowable waste items.

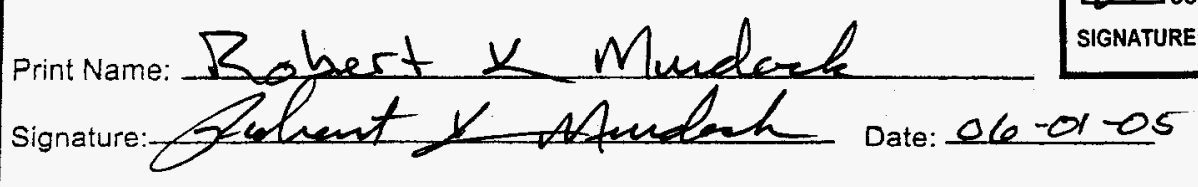

Radiation Survey Release for Waste Disposal $y$ RCT Initials This container/load is free of external radioactive

This container/load is exempt from survey due to

process knowledge and origin.
This container/load ie free of radioative (4) This container/load is free of radioaetive

SIGNATURE

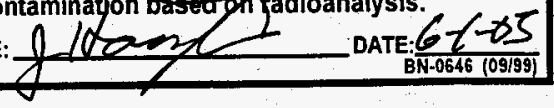

Note: Food waste, office trash and/or animal carcasses are considered not to contain added radioactivity, and therefore do not reguire a radiological clearance.

SWO USE ONLY

Load Weight (net from scale or estimate)

Signature of Certifier:

c. 


\section{SWO USE (Circle One Area) AREA \\ 23 \\ 6 \\ (9) \\ LANDFILL}

For waste characterization, approval, and/or assistance, contact Solid Waste Operation (SWO) at 5 -7898.

$$
\text { REQUIRED: WASTE GENERATOR INFORMATION }
$$

(This form is for rolloffs, dump trucks, and other onsite disposal of materials.)

Waste Generator: BuConstsuction (Mille Viuzic) Phone Number: 567326

Waste Category: (check one) $\square$ Commercial $\square$ Industrial

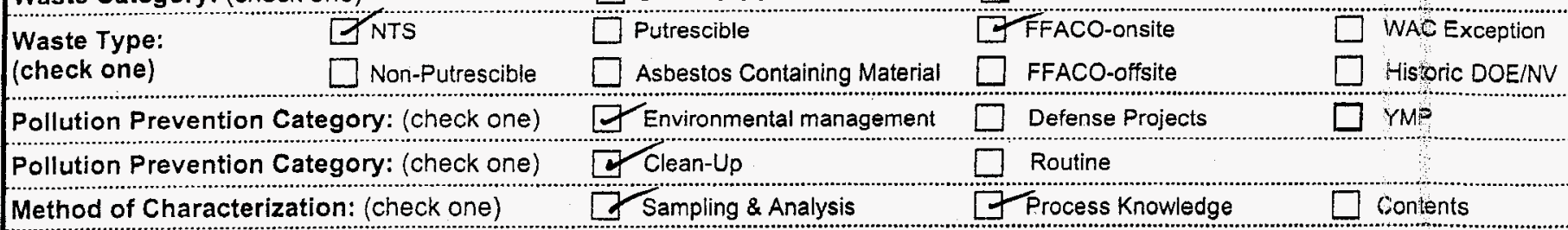

Prohibited Waste $\quad$ Radioactive waste; RCRA waste; Hazardous waste; Free liquids, PCBs above TSCA regulatory levels-, and Medical

at all three NTS landfills: wastes (needles, sharps, bloody clothing).

Additional Prohibited Waste Sewage Sludge; Animal carcasses-, Wet garbage (food waste); and Friable asbestos at the Area 9 U10c Landfill:

\section{REQUIRED: WASTE CONTENTS ALLOWABLE WASTES}

Check all allowable wastes that are contained within this load:

NOTE: Waste disposed at the Area 6 Hydrocarbon Landfill must have come into contact with petroleum hydrocarbons or coolants such as: gasoline (no benzene, lead); jet fuel; diesel fuel; lubricants and hydraulics; kerosene; asphaltic petroleum hydrocarbon; and ethylene glycol.

Acceptable waste at any NTS landfill: $\quad \square$ Paper $\quad \square$ Rocks / unaltered geologic materials $\square$ Emptycontainers
$\square$ Asphalt
TMetal
$\square$ Wood
$\square$ Soil
$\square$ Rubber (excluding tires)
4 Demoltion debris
TPlastic
$\checkmark$ Wire
$\square$ Cable $\square$ Cloth
$\square$ Insulation (non-Asbestosform)
TEemert \& concrete

$\square$ Manufactured items: (swamp coolers, furniture, rugs, carpet, electronic components, PPE, etc.)

Additional waste accepted at the Area 23 Mercury Landfill: $\square$ Office waste $\square$ Food Waste $\square$ Animal Carcasses

$\square$ Asbestos: $\square$ Friable $\square$ Non-Friable (contact SWO if regulated load) Quantity:

Additional waste accepted at the Area 9 U10c Landfill:
$\square$ Non-friable asbestos
$\square$ Drained automobiles and military vehicles
$\square$ Light ballasts (contact SWO)
Drained fuel filters (gas \& diesel)
Solid fractions from sand/oil/water separators
$\square$ Hydrocarbons (contact SWO)
Other
Deconned Underground and Above Ground
Additional waste accepted at the Area 6 Hydrocarbon Landfill:

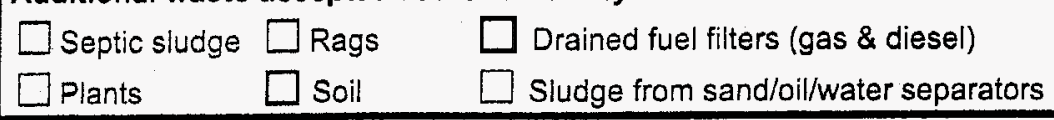
$\square$ Tanks
Other
$\square$ Crushed non-terne plated oil filters REQUIRED: WASTE GENERATOR SIGNATURE

Initials: (If initialed, no radiological clearance is necessary.)

The above mentioned waste was generated outside of a Controlled Wast knowledge, does not contain radiological materials.

To the best of my knowledge, the waste described above contains only tI site. I have verified this through the waste characterization method identi prohibited and allowable waste items.

Print Name: Rolvert k Murdock

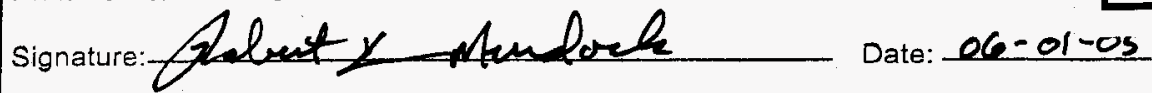

Radiation Survey Release for Waste Disposal RCT Initials RCT Inis containerlload is free of external radioactive contamination.

This container/load is exempt from survey due to process knowledge and origin.

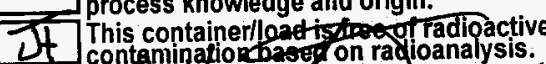
SIGNATURE: th

Note: Food waste, office trash and/or animal carcasses are considered not to contain added radioactivity, and therefore de not require a radiological clearance.

SWO USE ONLY
Load Weight (net from scale or estimate): 29 signature of certifier: 


\section{Bechtel Nevada NTS Landfill Load Verification \\ (Waste definitions are available on page 2)}

\begin{tabular}{llllll}
\hline SWO USE (Circle One Area) AREA & 23 & 6 & 9 & LANDFILL
\end{tabular}

For waste characterization, approval, and/or assistance, contact Solid Waste Operation (SWO) at 5 -7 898.

\section{REQUIRED: WASTE GENERATOR INFORMATION}

(This form is for rolloffs, dump trucks, and other onsite disposal of materials.)

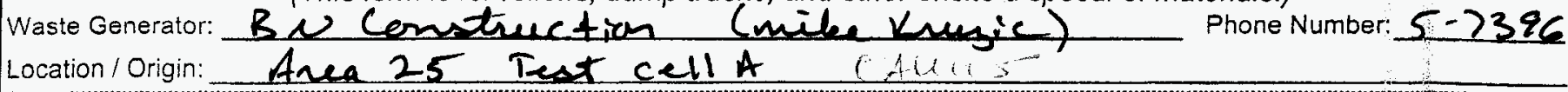

Waste Category: (check one) $\square$ Commercial $\square$ Tndustrial

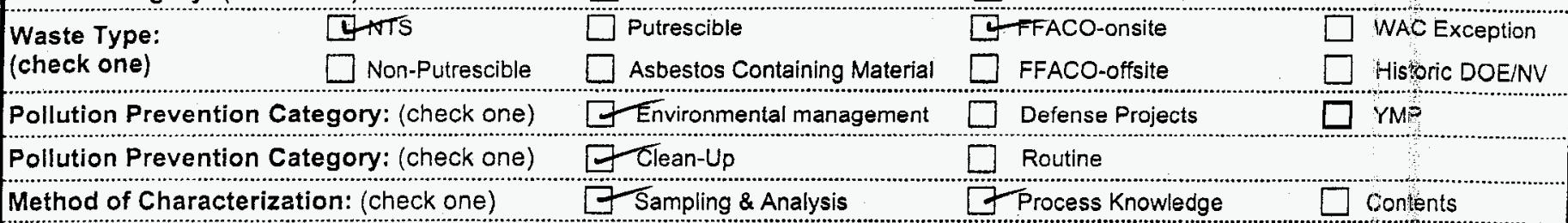

Prohibited Waste $\quad$ Radioactive waste; RCRA waste; Hazardous waste; Free liquids, PCBs above TSCA regulatory levels, and Medical

at all three NTS landfills: wastes (needles, sharps, bloody clothing).

Additional Prohibited Waste Sewage Sludge; Animal carcasses-, Wet garbage (food waste); and Friable asbestos at the Area 9 U10c Landfill:

\section{REQUIRED: WASTE CONTENTS ALLOWABLE WASTES}

Check all allowable wastes that are contained within this load:

NOTE: Waste disposed at the Area 6 Hydrocarbon Landfill must have come into contact with petroleum hydrocarbons or coolants such as: gasoline (no benzene, lead); jet fuel; diesel fuel; lubricants and hydraulics; kerosene: asphaltic petroleum hydrocarbon; and ethylene glycol.

Acceptable waste at any NTS landfill: $\square$ Paper $\square$ Rocks / unaltered geologic materials $\square$ Emptycontainers

$\begin{array}{lllll}\square \text { Asphalt } & \square \text { Metal } & \square \text { wood } & \square \text { soil } & \square \text { Rubber (excluding tires) }\end{array}$

$\square$ Manufactured items: (swamp coolers, furniture, rugs, carpet, electronic components, PPE, etc.)

Additional waste accepted at the Area 23 Mercury Landfill: $\square$ Office waste $\square$ Food Waste $\square$ Animal Carcasses

$\square$ Asbestos: $\square$ Friable $\square$ Non-Friable (contact SWO if regulated load) Quantity:

Additional waste accepted at the Area 9 U10c Landfill:

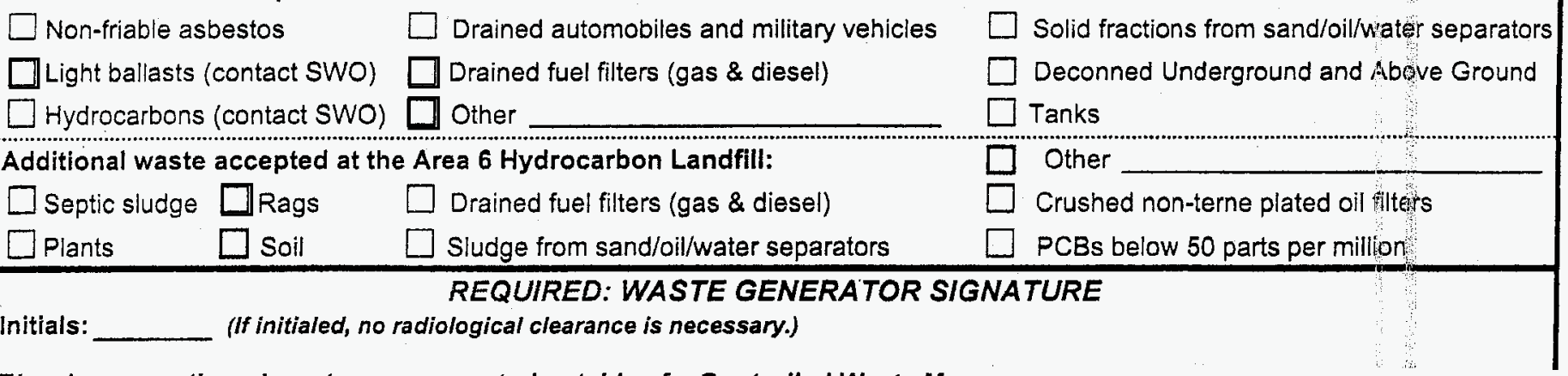

The above mentioned waste was generated outside of a Controlled Waste Mana! knowledge, does not contain radiological materials.

To the best of my knowledge, the waste described above contains only those $m$ site. I have verified this through the waste characterization method identified ab prohibited and allowable waste items.

Print Name: Robert $<$ Murdock Signature:flelexty Mardexh $06-01-05^{-}$

Radiation Survey Release for Waste Disposal RCT Initials

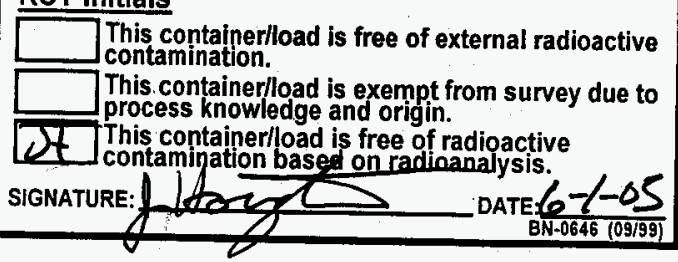

Note: Food waste, office trash and/or animal carcasses are considered not to contain added radioactivity, and therefore do not require a radiological clearance.

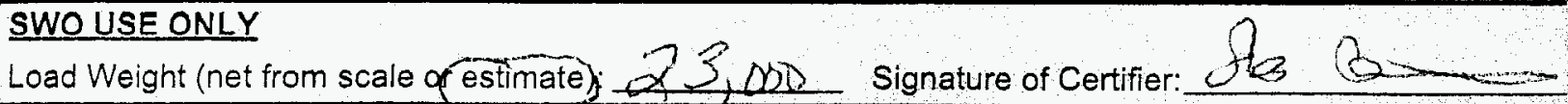


6

9

LANDFILL

For waste characterization, approval, and/or assistance, contact Solid Waste Operation (SWO) at 5 -7 398. REQUIRED: WASTE GENERATOR INFORMATION

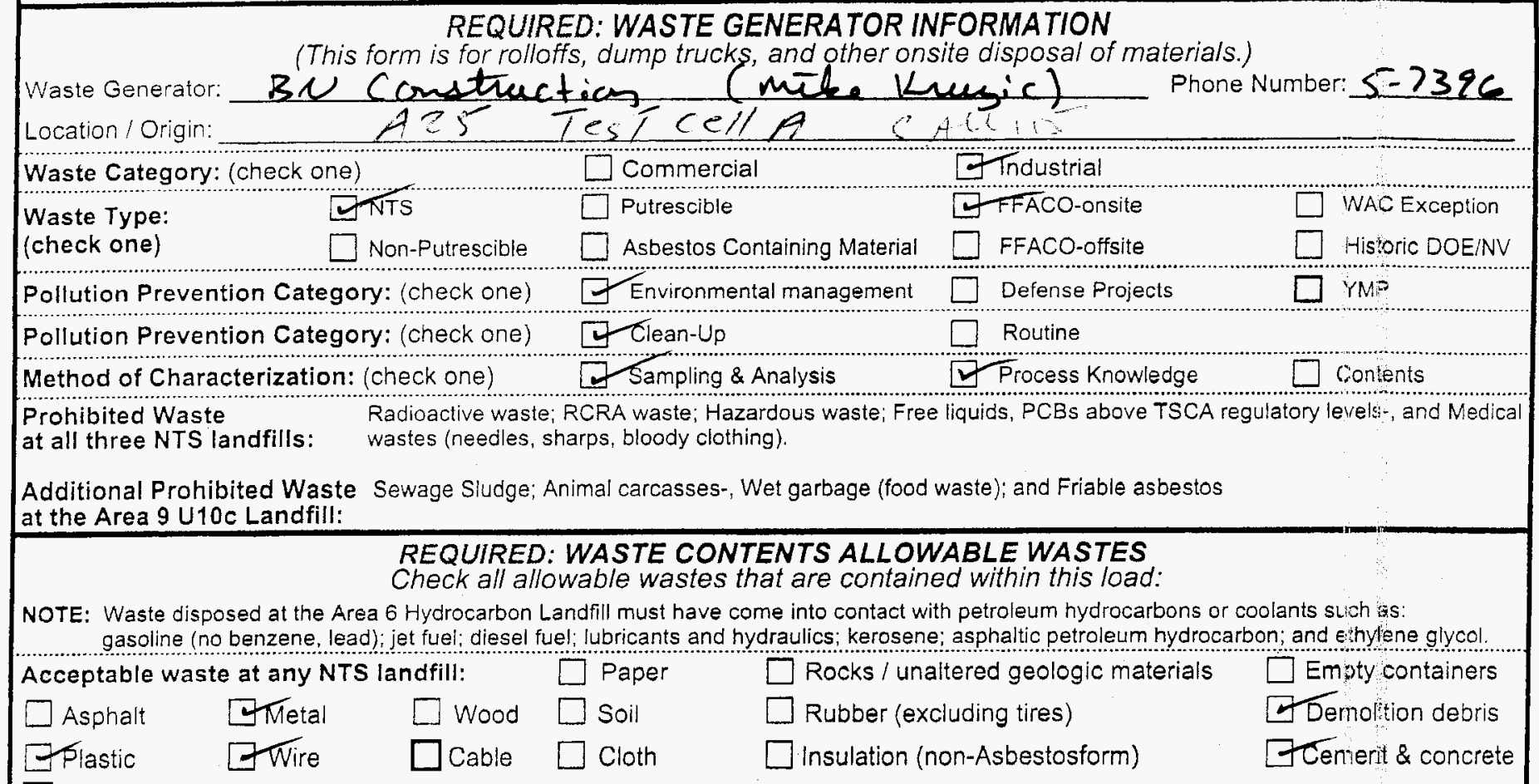

GPlastic $\square$ Wire $\square$ Cable $\square$ Cloth $\square$ Insulation (non-Asbestosform)

Additional waste accepted at the Area 23 Mercury Landfill: $\square$ Office waste $\square$ Food Waste. $\square$ Animal Carcasses

$\square$ Asbestos: $\square$ Friable $\square$ Non-Friable (contact SWO if regulated load) Quantity:

Additional waste accepted at the Area 9 U10c Landfill:
$\square$ Non-friable asbestos
$\square$ Drained automobiles and military vehicles
$\square$ Light ballasts (contact SWO)
[
Drained fuel filters (gas \& diesel)
Other
Solid fractions from sand/oil/water separators
$\square$ Hydrocarbons (contact SWO)
Deconned Underground and Above Ground
Additional waste accepted at the Area 6 Hydrocarbon Landfill:
$\square$ Septic sludge $\square$ Rags
$\square$ Plants $\square$ soil
$\square$ Drained fuel filters (gas \& diesel)
$\square$ Sludge from sand/oil/water separators
$\square$ Tanks
Initials:
REQUIRED: WASTE GENERATOR SIGNATURE
Other
$\square$ Crushed non-terne plated oil iilters
$\square$ PCBs below 50 parts per million (If initialed, no radiological clearance is necessary.)

The above mentioned waste was generated outside of a Controlled Waste Manage knowledge, does not contain radiological materials.

To the best of my knowledge, the waste described above contains only those mat site. I have verified this through the waste characterization method identified abov prohibited and allowable waste items.

Print Name: Robert $L$ Murdock

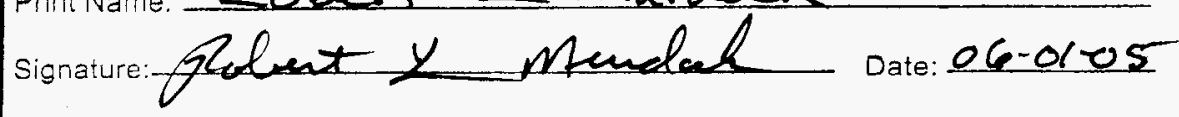

Radiation Survey Release for Waste Dispos: RCT Initials

This container/load is free of external radioactive contamination.

This container/load is exempt from survey due to process knowledge and origin. II This containerlload is free of radioactive

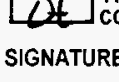
DATE: $6 r-\sigma_{2}^{c}$

Note: Food waste, office trash and/or animal carcasses are considered not to contain added radioactivity, and therefore do not require a radiological clearance.

SWO USE ONLY

Load Weight (net from scale or estimate): $3 \times 2,20$

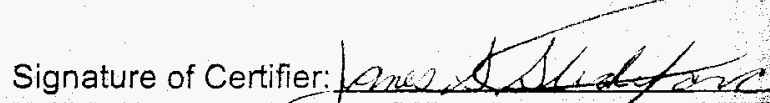


Driver Name: Warren Morris Trucle: 682 -0699A

\section{Bechtel Nevada NTS Landfill Load Verification}

(Waste definitions are available on page 2)

\begin{tabular}{lllll}
\hline SWO USE (Circle One Area) AREA & 23 & 6 & 9
\end{tabular}

For waste characterization, approval, and/or assistance, contact Solid Waste Operation (SWO) at $5-7398$.

\section{REQUIRED: WASTE GENERATOR INFORMATION}

(This form is for rolloffs, dymp trucks, and other onsite disposal of materials.)

Waste Generator: BN Conttruction (milue Kungic) Phone Number: $5-3326$ Location / Origin: thea 25 Tent Cell t $\quad$ All : J

Waste Category: (check one) $\square$ Commercial 7 industrial

Waste Type:

$\square$ NTS

(check one)

$\square$ Non-Putrescible

$\square$ Asbestos Containing Material

4 FFACO-onsite

WAC Exception

Pollution Prevention Category: (check one)

Pollution Prevention Category: (check one)

7 Environmentai management

FFACO-offsite

Historic DOE/NV

Method of Characterization: (check one)

7 Clean-Up

Method of Characterization: (check one)

7 Sampling \& Analysis

Defense Projects

YMP

Prohibited Waste

Radioactive waste; RCRA waste; Hazardo
wastes (needles, sharps, bloody clothing).

Additional Prohibited Waste Sewage Sludge; Animal carcasses-, Wet garbage (food waste); and Friable asbestos at the Area 9 U10 L Landfill:

\section{REQUIRED: WASTE CONTENTS ALLOWABLE WASTES}

Check all allowable wastes that are contained within this load:

NOTE: Waste disposed at the Area 6 Hydrocarbon Landfill must have come into contact with petroleum hydrocarbons or coolants such as: gasoline (no benzene, lead); jet fuel; diesel fuel; lubricants and hydraulics; kerosene; asphaltic petroleum hydrocarbon; and ethylene giycol.

Acceptable waste at any NTS landfill: $\square$ Paper $\square$ Rocks / unaltered geologic materials $\square$ Empty containers
$\square$ Asphalt
Metal
$\square$ Wood
Soil
$\square$ Rubber (excluding tires)
J Plastic
Wwire
$\square$ Cable $\square$ Cloth
$\square$ Insulation (non-Asbestosform)
7 bemolition debris

$\square$ Manufactured items: (swamp coolers, furniture, rugs, carpet, electronic components, PPE, etc.)

Additional waste accepted at the Area 23 Mercury Landfill: $\square$ Office waste $\square$ Food Waste $\square$ Animal Carcasses

$\square$ Asbestos: $\square$ Friable $\square$ Non-Friable (contact SWO if regulated load) Quantity:

Additional waste accepted at the Area 9 U10c Landfill:

$\begin{array}{ll}\square \text { Non-friable asbestos } & \square \text { Drained automobiles and military vehicles } \\ \square \text { Light ballasts (contact SWO) } \square \text { Drained fuel filters (gas \& diesel) } \\ \square \text { Hydrocarbons (contact SWO) } \square \text { Other }\end{array}$

Additional waste accepted at the Area 6 Hydrocarbon Landfill:

$\square$ Septic sludge $\square$ Rags

$\square$ Plants

$\square$ Soil $\square$ Drained fuel filters (gas \& diesel)

$\square$ Sludge from sand/oil/water separators
Solid fractions from sand/oil/water separators

Deconned Underground and Above Ground

Tanks

Other

Crushed non-terne plated oil filters

PCBs below 50 parts per million

Initials: REQUIRED: WASTE GENERATOR SIGNATURE

(If initialed, no radiological clearance is necessary.)

The above mentioned waste was generated outside of a Controlled Waste $M$ knowledge, does not contain radiological materials.

To the best of my knowledge, the waste described above contains only thos site. I have verified this through the waste characterization method identifie prohibited and allowable waste items.

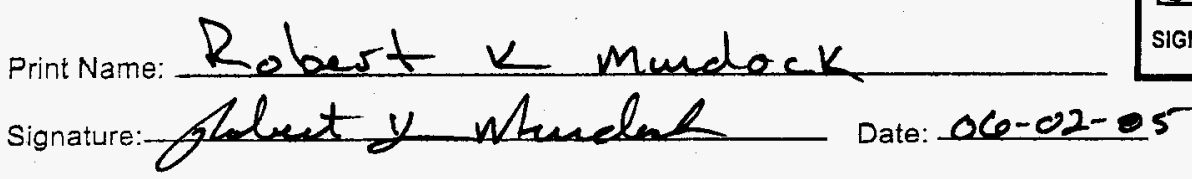

Radiation Survey Release for Waste Disposal RCT Initials

This containerlload is free of external radioactive contamination.

This container/load is exempt from survey due to process knowledge and origin. $J L R$ This container/load is free of radioactive SIGNATURE: Aosepho/Conley DATE: $6 / 2 / 05$

Note: Food waste, office trash and/or animal carcasses are considered not to contain added radioactivity, and therefore do not require a radiological clearance.

SWO USE ONLY

Load Weight (net from scale of estimate): 20,202 


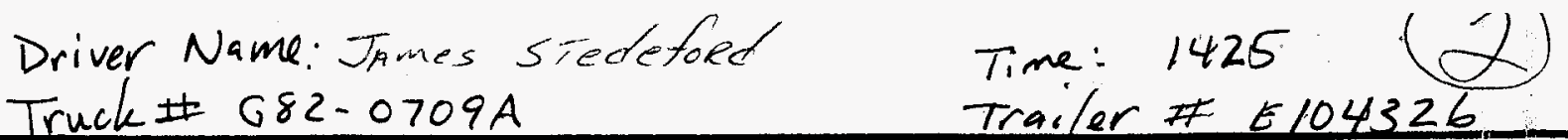

\section{Bechtel Nevada NTS Landfill Load Verification \\ (Waste definitions are available on page 2)

\begin{tabular}{llllll}
\hline SWO USE (Circle One Area) AREA & 23 & 6 & 9 & LANDFILL
\end{tabular}

For waste characterization, approval, and/or assistance, contact Solid Waste Operation (SWO) at 5 -7898.

REQUIRED: WASTE GENERATOR INFORMATION

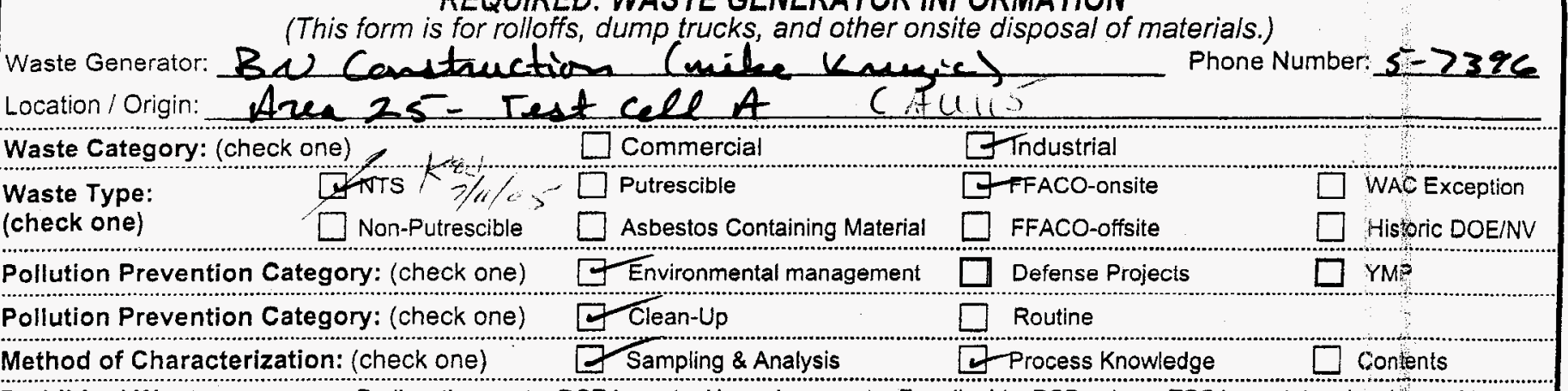

Prohibited Waste Radioactive waste; RCRA waste; Hazardous waste; Free liquids, PCBs above TSCA regulatory levels: and Medical

at all three NTS landfills: wastes (needles, sharps, bloody clothing).

Additional Prohibited Waste Sewage Sludge; Animal carcasses-, Wet garbage (food waste); and Friable asbestos at the Area 9 U10c Landfill:

REQUIRED: WASTE CONTENTS ALLOWABLE WASTES
Check all allowable wastes that are contained within this load:

NOTE: Waste disposed at the Area 6 Hydrocarbon Landfill must have come into contact with petroleum hydrocarbons or coolants sLith as: gasoline (no benzene, lead); jet fuel; diesel fuel; lubricants and hydraulics; kerosene; asphaltic petroleum hydrocarbon; and ethyene glycol. Acceptable waste at any NTS landfill: $\quad \square$ Paper $\quad \square$ Rocks / unaltered geologic materials $\square$ Emoty containers
$\square$ Asphalt
$\square$ Metal
Thood
$\square$ Soil
$\square$ Rubber (excluding tires)
$\square$ Insulation (non-Asbestosform)
TDernolition debris Cenerit \& concrete

Manufactured items: (swamp coolers, furniture, rugs, carpet, electronic components, PPE, etc.)

Additional waste accepted at the Area 23 Mercury Landfill: $\square$ Office waste $\square$ Food Waste $\square$ Animal Carcasses

$\square$ Asbestos: $\square$ Friable $\square$ Non-Friable (contact SWO if regulated load) Quantity:

Additional waste accepted at the Area 9 U10c Landfill:
$\square$ Non-friable asbestos
$\square$ Drained automobiles and military vehicles
$\square$ Light ballasts (contact SWO) $\square$ Drained fuel filters (gas \& diesel)
Solid fractions from sand/oil/water separators
$\square$ Hydrocarbons (contact SWO) $\square$ Other
Additional waste accepted at the Area 6 Hydrocarbon Landfill:
$\square$ Septic sludge $\square$ Rags $\square$ Drained fuel filters (gas \& diesel)
$\square$ Plants $\square$ soil $\square$ sludge from sand/oil/water separators
REQUIRED: WASTE GENERATOR SIGNATURE
Initials: (If initialed, no radiological clearance is necessary.)

The above mentioned waste was generated outside of a Controlled Waste Management Area (CWMA) and to the trest of my knowledge, does not contain radiological materials.

To the best of my knowledge, the waste described above contains only those ma site. I have verified this through the waste characterization method identified abo prohibited and allowable waste items.

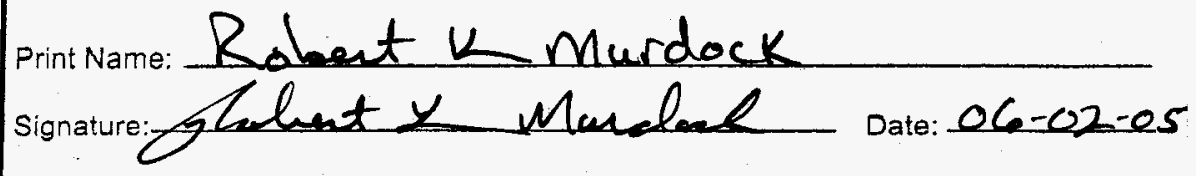

Note: Food waste, office trash and/or animal carcasses are considered not to contain a require a radiological clearance.

SWO USE ONLY

Load Weight (net from scale of estimate) 202600 signature of Certifier.

Radiation Survey Release for Waste Disposal RCT Initials

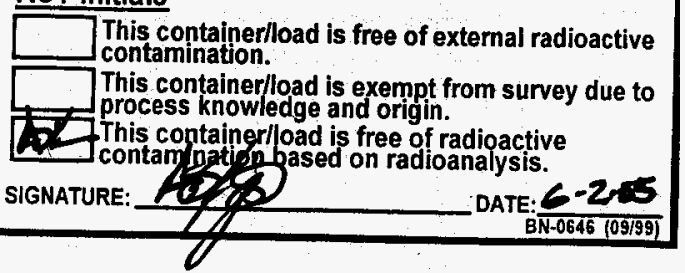

This container/load is free of external radioactive
contamination.

This container/load is exempt from survey due to process knowledge and origin.

This container/load is free of radioactive

DATE $6-2,5$

BN-0646 (09/99) 
Dsiver name: fitgorrow

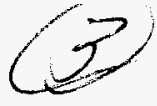

Survey F. O\& $-6 K=12+2-276$

Truek G82-069814

Trailui E 100035

\section{Bechtel Nevada}

NTS Landfill Load Verification

(Waste definitions are available on page 2)

\begin{tabular}{lllll}
\hline SWO USE (Circle One Area) AREA & 23 & 6 & (9) LANDFILL
\end{tabular}

For waste characterization, approval, and/or assistance, contact Solid Waste Operation (SWO) at 5 -7988.

REQUIRED: WASTE GENERATOR INFORMATION

(This form is for rolloffs, dump trucks, and other onsite disposal of materials.)

Waste Generator: BU Conctruction (múle Kuyic) Phone Number: $5-2376$ Location/Origin: Anea 25- Intcell ot P.ALis

Waste Category: (check one) $\quad$ [ Commercial

Waste Type:

(check one)

TNTS

4 FFACO-onsite

WAC Exception

$\square$ Non-Putrescible $\square$ Asbestos Containing Materia

$\square$ FFACO-offsite

Historic DOE/NV

Pollution Prevention Category: (check one)

$\checkmark$ Environmental management

Defense Projects

$Y M P$

Pollution Prevention Category: (check one)

4 Clean-Up

Method of Characterization: (check one)

7 Sampling \& Analysis

Routine

Process Knowledge

Radioactive waste; RCRA waste; Hazardous waste; Free liquids, PCBs above TSCA regulatory levels:, and Medica at all three NTS landfills: $\quad$ wastes (needles, sharps, bloody clothing).

Additional Prohibited Waste Sewage Siudge; Animal carcasses-, Wet garbage (food waste); and Friable asbestos

at the Area 9 U10c Landfill:

REQUIRED: WASTE CONTENTS ALLOWABLE WASTES

Check all allowable wastes that are contained within this load:

NOTE: Waste disposed at the Area 6 Hydrocarbon Landfill must have come into contact with petroleum hydrocarbons or coolants sulch as: gasoline (no benzene, lead); jet fuel; diesel fuel; lubricants and hydraulics; kerosene; asphaltic petroleum hydrocarbon; and exhylene glycol.

Acceptable waste at any NTS landfill: $\quad \square$ Paper $\quad \square$ Rocks / unaltered geologic materials

$\square$ Asphalt $\quad \square$ Metal $\quad \square$ wood $\square$ Soil $\quad \square$ Rubber (excluding tires)

$\square$ Plastic $\quad \square$ Wire $\quad \square$ Cable $\square$ Cloth $\square$ Insulation (non-Asbestosform)

$\square$ Manufactured items: (swamp coolers, furniture, rugs, carpet, electronic components, PPE, etc.)

Additional waste accepted at the Area 23 Mercury Landfill: $\square$ Office waste $\square$ Food Waste $\square$ Animal Carcasses

$\square$ Asbestos: $\square$ Friable $\square$ Non-Friable (contact SWO if regulated load) Quantity:

Additional waste accepted at the Area 9 U10c Landfill:

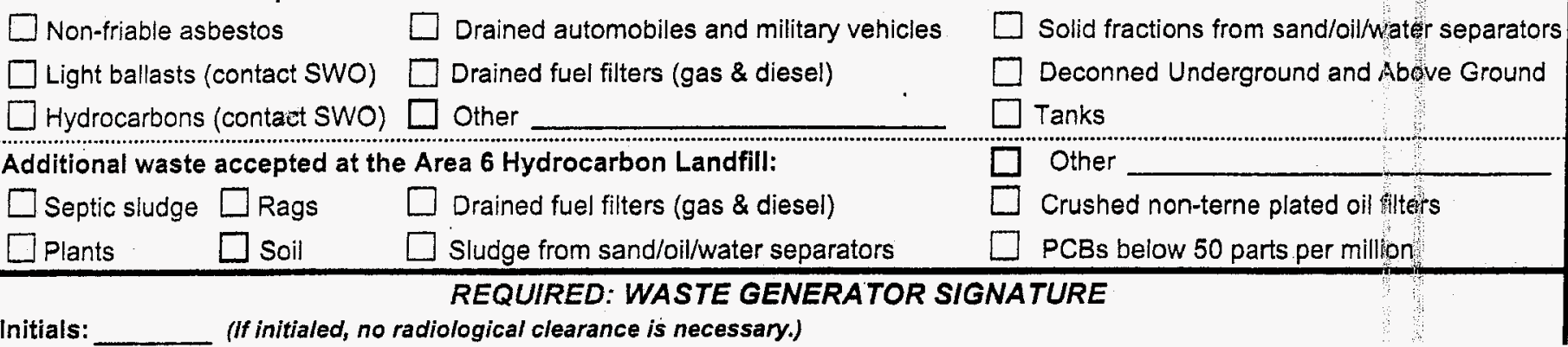

The above mentioned waste was generated outside of a Controlled Waste knowledge, does not contain radiological materials.

To the best of my knowledge, the waste described above contains only the site. I have verified this through the waste characterization method identifi prohibited and allowable waste items.

Print Name: Robert KMurdock

Radiation Survey Release for Waste Disposal RCT Initials

$\square$ This container/load is free of external radioactive contamination.

This container/load is exempt from survey due to process knowledge and origin.

IR This container/load is free of radioactive $J L R$ contamination based on radiognalysis. SIGNATURE

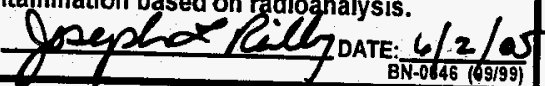

Signature: zoluent y Mfudele Date:06-02.05

Note: Food waste, office trash and/or animal carcasses are considered not to contain added radioactivity, and therefore de not require a radiological clearance.

SWO USE ONLY

Load Weight (net from scale or estimate): $2700 \mathrm{~d}$ 


\section{Bechtel Nevada NTS Landfill Load Verification}

(Waste definitions are available on page 2)

\begin{tabular}{lllll}
\hline SWO USE (Circle One Area) AREA & 23 & 6 & 9 & LANDFILL
\end{tabular}

For waste characterization, approval, and/or assistance, contact Solid Waste Operation (SWO) at $5-7898$.

REQUIRED: WASTE GENERATOR INFORMATION

(This form is for rolloffs, dump trucks, and other onsite disposal of materials.)

\begin{tabular}{|c|c|c|c|c|}
\hline \multicolumn{2}{|c|}{ Waste Category: (check one) } & $\square$ Commercial & $\exists$ Industrial & \\
\hline \multirow{2}{*}{$\begin{array}{l}\text { Waste Type: } \\
\text { (check one) }\end{array}$} & FNTS R $9 \% .1 \%$ & $\square$ Putrescible & GFFACO-onsite & $\square$ WAE Exception \\
\hline & $\square$ Non-Putrescible & $\square$ Asbestos Containing Material & $\square$ FFACO-offsite & $\square$ Historic DOE/NV \\
\hline \multicolumn{2}{|c|}{ Pollution Prevention Category: (check one) } & 7 Environmental management & $\square$ Defense Projects & $\square Y M^{\circ}$ \\
\hline \multicolumn{2}{|c|}{ Pollution Prevention Category: (check one) } & IClean-Up & $\square$ Routine & \\
\hline \multicolumn{2}{|c|}{ Method of Characterization: (check one) } & 7 Sampling \& Analysis & 3 Process Knowledge & $\square$ Contents \\
\hline
\end{tabular}

Prohibited Waste $\quad$ Radioactive waste; RCRA waste; Hazardous waste; Free liquids, PCBs above TSCA regulatory levels, and Medical at all three NTS landfills: wastes (needles, sharps, bloody clothing).

Additional Prohibited Waste Sewage Sludge; Animal carcasses-, Wet garbage (food waste); and Friable asbestos at the Area 9 U10c Landfill:

REQUIRED: WASTE CONTENTS ALLOWABLE WASTES

Check all allowable wastes that are contained within this load:

NOTE: Waste disposed at the Area 6 Hydrocarbon Landfill must have come into contact with petroleum hydrocarbons or coolants such as: gasoline (no benzene, lead); jet fuel: diesel fuel; lubricants and hydraulics; kerosene; asphaltic petroleum hydrocarbon; and ethylene glycol.

Acceptable waste at any NTS landfill: $\quad \square$ Paper $\quad \square$ Rocks / unaltered geologic materials $\square$ Emotycontainers

$\square$ Asphait $\quad \square$ Metal $\quad \square$ wood $\square$ Soil $\square$ Rubber (excluding tires)

GPlastic $\square$ Wire $\square$ Cable $\square$ Cloth $\square$ insulation (non-Asbestosform)

Temolition debris

$\square$ Manufactured items: (swamp coolers, furniture, rugs, carpet, electronic components, PPE, etc.)

Additional waste accepted at the Area 23 Mercury Landfill: $\square$ Office waste $\square$ Food Waste $\square$ Animal Carcasses

Asbestos: $\square$ Friable $\square$ Non-Friable (contact SWO if regulated load) Quantity:

Additional waste accepted at the Area 9 U10 L Landfill:
$\square$ Non-friable asbestos
$\square$ Drained automobiles and military vehicles
Light ballasts (contact SWO)
Drained fuel filters (gas \& diesel)
Solid fractions from sand/oil/water separators
Hydrocarbons (contact SWO)
Other
Deconned Underground and Above Ground
Additional waste accepted at the Area 6 Hydrocarbon Landfill:
$\square$ Septic sludge $\square$ Rags
$\square$ Plants $\quad \square$ Soil
Drained fuel filters (gas \& diesel)
Tanks
Sludge from sand/oil/water separators
Other
$\square$. Crushed non-terne plated oil filters
Initials: (If initialed, no radiological clearance is necessary.)

\section{REQUIRED: WASTE GENERATOR SIGNATURE}

The above mentioned waste was generated outside of a Controlled Wast knowledge, does not contain radiological materials.

To the best of my knowledge, the waste described above contains only $t$ site. I have verified this through the waste characterization method identi prohibited and allowable waste items.

Print Name: Robert $k$ Murdock

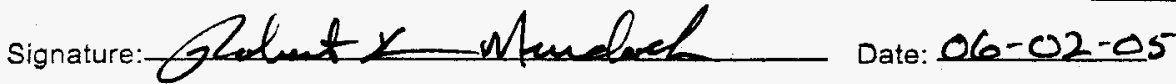

Radiation Survey Release for Waste Disposal RCT Initials

$\square$ This containeriload is free of external radioactive contamination.

This container/load is exempt from survey due to process knowledge and origin.

J4 This container/load is free of radioactive

SIGNATURE

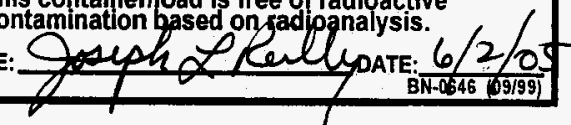

Note: Food waste, office trash and/or animal carcasses are considered not to contain added radioactivity, and therefore de not reguire a radiological clearance.

SWO USE ONLY

Load Weight (net from scale or estimate): 25000

Signature of Certifier:

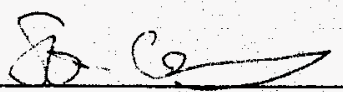


Ariver: / ArRliA LT7tomis

Treek: $G 82-0>31 A$

Bechtel Nevada NTS Landfill Load Verification

(Waste definitions are available on page 2)

\begin{tabular}{lllll}
\hline SWO USE (Circle One Area) AREA & 23 & 6 & (9) & LANDFILL
\end{tabular}

For waste characterization, approval, and/or assistance, contact Solid Waste Operation (SWO) at 5 -7398.

\section{REQUIRED: WASTE GENERATOR INFORMATION}

(This form is for rolloffs, dump trucks, and other onsite disposal of materials.)

Waste Generator: Bu Cendrection

Location/Origin: Area 25 Test

Waste Category: (check one)

Waste Type:

4 NTS 2 .

$\square$ Non-Putrescible

cuiles

Phone Number: $5-2326$

(check one)

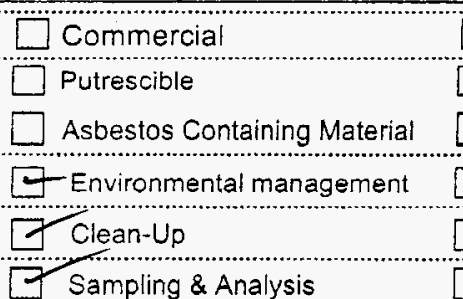

\begin{tabular}{l}
\hline Tndustrial \\
$\square$ FFACO-onsite \\
$\square$ FFACO-offsite
\end{tabular}

Pollution Prevention Category: (check one)

Pollution Prevention Category: (check one)

Method of Characterization: (check one)

Sampling \& Analysis

Radioactive waste; RCRA waste; Hazardous waste; Free liquids, PCBs above TSCA regulatory levels-, and Medica

Prohibited Waste

wastes (needles, sharps, bloody clothing).

Additional Prohibited Waste Sewage Sludge; Animal carcasses-, Wet garbage (food waste); and Friable asbestos

at the Area 9 U10c Landfill:

REQUIRED: WASTE CONTENTS ALLOWABLE WASTES
Check all allowable wastes that are contained within this load:

NOTE: Waste disposed at the Area 6 Hydrocarbon Landfill must have come into contact with petroleum hydrocarbons or coolants stich as: gasoline (no benzene, lead); jet fuel: diesel fuel: lubricants and hydraulics; kerosene; asphaltic petroleum hydrocarbon; and ethylene glycol

Acceptable waste at any NTS landfill: $\quad \square$ Paper $\quad \square$ Rocks / unaltered geologic materials $\square$ Empty containers

$\square$ Asphalt $\quad \square$ Metal $\square$ wood $\square$ soil $\square$ Rubber (excluding tires)

$\square$ Plastic $\quad \square$ Wire $\quad \square$ Cable $\square$ Cloth

$\square$ Insulation (non-Asbestosform)

Temoltion debris

$\square$ Manufactured items: (swamp coolers, furniture, rugs, carpet, electronic components, PPE, etc.)

Additional waste accepted at the Area 23 Mercury Landfill: $\square$ Office waste $\square$ Food Waste $\square$ Animal Carcasses

$\square$ Asbestos: $\square$ Friable $\square$ Non-Friable (contact SWO if regulated load) Quantity:

Additional waste accepted at the Area 9 U10c Landfill:

$\square$ Non-friable asbestos
$\square$ Light ballasts (contact SWO) $\square$ Drained automobiles and military vehicles
$\square$ Hydrocarbons (contact SWO) $\square$ Other

Additional waste accepted at the Area 6 Hydrocarbon Landfill:
$\square$ Septic sludge $\square$ Rags
$\square$ Drained fuel filters (gas \& diesel)
$\square$ Plants $\quad \square$ soil
$\square$ Sludge from sand/oil/water separators

Solid fractions from sand/oil/water separators

$\square$ Deconned Underground and Above Ground

$\square$ Tanks

REQUIRED: WASTE GENERATOR SIGNATURE

Initials: (If initialed, no radiological clearance is necessary.)

The above mentioned waste was generated outside of a Controlled Waste knowledge, does not contain radiological materials.

To the best of my knowledge, the waste described above contains only thc site. I have verified this through the waste characterization method identifi prohibited and allowable waste items.

Print Name: Robbert K Murdock

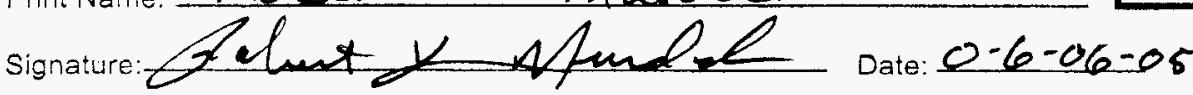

Radiation Survey Release for Waste Disposal RCT Initials

This container/load is free of external radioactive contamination.

This container/load is exempt from survey due to process knowledge and origin.

Tht This container/load is free of radioactive 1 contamination based op radiganalysis. SIGNATURE:

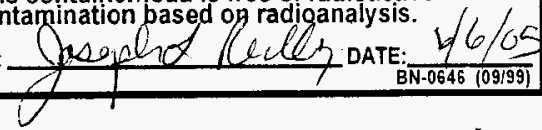

Note: Food waste, office trash and/or animal carcasses are considered not to contain added radioactivity, and therefore do not require a radiological clearance.

SWO USE ONLY

Load Weight (net from scale or estimate): 25,50

Signature of Certifier: 
Driver: Warren Morís

Fruck: G82-0699A

Bechtel Nevada NTS Landfill Load Verification

(Waste definitions are available on page 2)

\begin{tabular}{lllll}
\hline SWO USE (Circle One Area) AREA & 23 & 6 & (9) LANDFILL
\end{tabular}

For waste characterization, approval, and/or assistance, contact Solid Waste Operation (SWO) at 6-7898.

REQUIRED: WASTE GENERATOR INFORMATION

(This form is for rolloffs, dump trucks, and other onsite disposal of materials.)

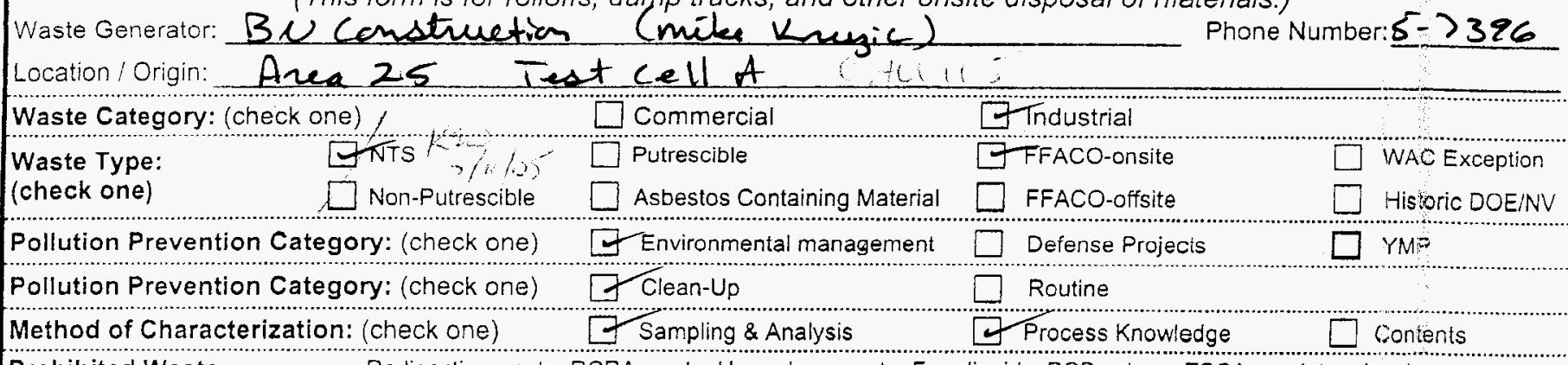

Prohibited Waste $\quad$ Radioactive waste; RCRA waste; Hazardous waste; Free liquids, PCBs above TSCA regulatory levels-, and Medical at all three NTS landfills: wastes (needles, sharps, bloody clothing).

Additional Prohibited Waste Sewage Sludge; Animal carcasses-, Wet garbage (food waste); and Friable asbestos

at the Area 9 U10c Landfill:

REQUIRED: WASTE CONTENTS ALLOWABLE WASTES

Check all allowable wastes that are contained within this load:

NOTE: Waste disposed at the Area 6 Hydrocarbon Landfill must have come into contact with petroleum hydrocarbons or coolants sLich as: gasoline (no benzene, lead); jet fuel; diesel fuel; lubricants and hydraulics; kerosene; asphaltic petroleum hydrocarbon; and ethylene glycol.

Acceptable waste at any NTS landfill: $\quad \square$ Paper $\square$ Rocks / unaltered geologic materials $\square$ Emptycontainers

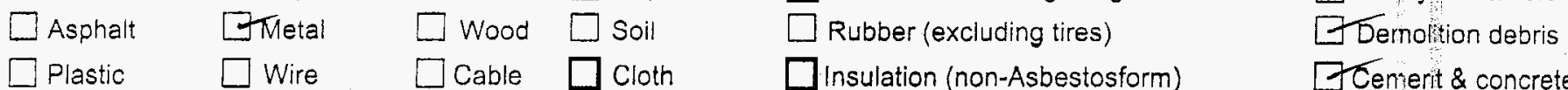

$\square$ Manufactured items: (swamp coolers, furniture, rugs, carpet, electronic components, PPE, etc.)

Additional waste accepted at the Area 23 Mercury Landfill: $\square$ Office waste $\square$ Food Waste $\square$ Animal Carcasses

$\square$ Asbestos: $\square$ Friable $\square$ Non-Friable (contact SWO if regulated load) Quantity:

Additional waste accepted at the Area 9 U10c Landfill:

$\square$ Non-friable asbestos $\square$ Drained automobiles and military vehicles

$\square$ Light ballasts (contact SWO) $\square$ Drained fuel filters (gas \& diesel)

Solid fractions from sand/oil/water separators

$\square$ Hydrocarbons (contact SWO) $\square$ Other

Additional waste accepted at the Area 6 Hydrocarbon Landfill:

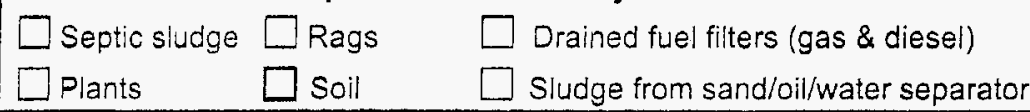

$\square$ Deconned Underground and Above Ground

$\square$ Tanks

REQUIRED: WASTE GENERATOR SIGNATURE

Initials:

(If initialed, no radiological clearance is necessary.)

The above mentioned waste was generated outside of a Controlled Wast knowledge, does not contain radiological materials.

To the best of my knowledge, the waste described above contains only t: site. I have verified this through the waste characterization method ident: prohibited and allowable waste items.

Print Name: Robert Murdock

signature:-ghtut $y$ Afuen Date: $06-06-05$

Radiation Survey Release for Waste Disposal RCT Initials

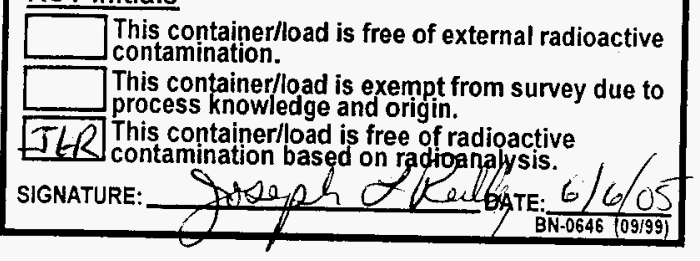

Note: Food waste, office trash and/or animai carcasses are considered not to contain added radioactivity, and therefore do not require a radiological clearance.

SWO USE ONLY

Load Weight (net from scale or estimate):

Signature of Certifie 


\section{$\begin{array}{lllll}\text { SWO USE (Circle One Area) AREA } & 23 & 6 & \text { (9) LANDFILL }\end{array}$}

For waste characterization, approval, and/or assistance, contact Solid Waste Operation (SWO) at $5-7398$.

\section{REQUIRED: WASTE GENERATOR INFORMATION}

(This form is for rolloffs, dump trucks, and other onsite disposal of materials.) Waste Generator: Bu Construction (Mike knuze) Phone Number:5-7396

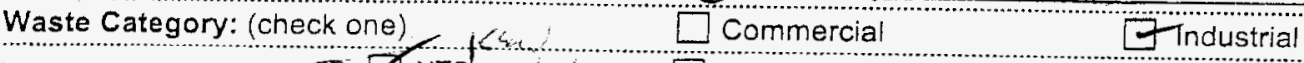

Waste Type: - JNTS J/16Y $\square$ Putrescible 7 FFACO-onsite

(check one) $\square$ Non-Putrescible $\square$ Asbestos Containing Material $\square$ FFACO-offsite

Pollution Prevention Category: (check one)

Pollution Prevention Category: (check one)

Environmental management

Clean-Up

7 Sampling \& Analysis

Defense Projects

WAC Exception

Method of Characterization: (check one)

Prohibited Waste

at all three NTS landfills:

Historic DOE/NV

wastes (needles, sharps, bloody clothing).

Additional Prohibited Waste Sewage Sludge; Animal carcasses-, Wet garbage (food waste); and Friable asbestos
at the Area 9 U10c Landfill:

REQUIRED: WASTE CONTENTS ALLOWABLE WASTES

Check all allowable wastes that are contained within this load:

NOTE: Waste disposed at the Area 6 Hydrocarbon Landfill must have come into contact with petroleum hydrocarbons or coolants stich as: gasoline (no benzene, lead); jet fuel; diesel fuel: lubricants and hydraulics; kerosene; asphaltic petroleum hydrocarbon; and ethylene glycol.

Acceptable waste at any NTS landfill: $\square$ Paper $\square$ Rocks / unaltered geologic materials $\square$ Empty containers
$\square$ Asphalt
Metal
$\square$ Wood
Soil
$\square$ Rubber (excluding tires)
$\square$ Plastic
Wire
$\square$ Cable
Cloth
$\square$ Insulation (non-Asbestosform)
$\checkmark$ Demoltion debris
Generit \& concrete

$\square$ Manufactured items: (swamp coolers, furniture, rugs, carpet, electronic components, PPE, etc.)

Additional waste accepted at the Area 23 Mercury Landfill: $\square$ Office waste $\square$ Food Waste $\square$ Animal Carcasses

$\square$ Asbestos: $\square$ Friable $\square$ Non-Friable (contact SWO if regulated load) Quantify:

Additional waste accepted at the Area 9 U10c Landfill:

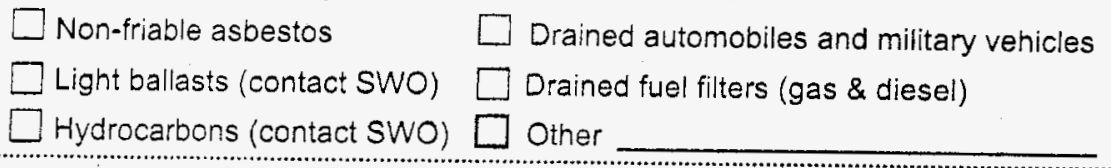

Additional waste accepted at the Area 6 Hydrocarbon Landfill:

$\square$ Septic sludge $\square$ Rags $\quad \square$ Drained fuel filters (gas \& diesel)

$\square$ Plants $\square$ Soil $\square$ sludge from sand/oil/water separators

Initials: (If initialed, no radiological clearance is necessary.)

The above mentioned waste was generated outside of a Controlled Was knowledge, does not contain radiological materials.

To the best of my know/edge, the waste described above contains only site. I have verified this through the waste characterization method ider prohibited and allowable waste items.

Print Name: Robert K Murdock

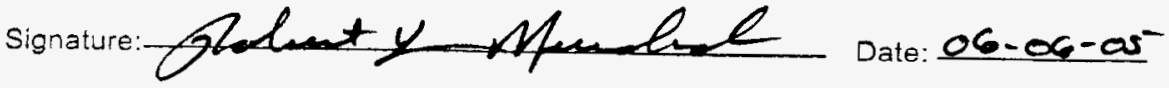

Note: Food waste, office trash and/or animal carcasses are considered not to contain added radioactivity, and therefore do not
require a radiological clearance. SWO USE ONLY

Load Weight (net from scale(or estimate) : Signature of Certifier:

PCBs below 50 parts per million

Solid fractions from sand/oil/water separators

$\square$ Deconned Underground and Above Ground 7 Tanks

0 Other

$\square$ Crushed non-terne plated oil illters Radiation Survey Release for Waste Disposal
RCT Initials

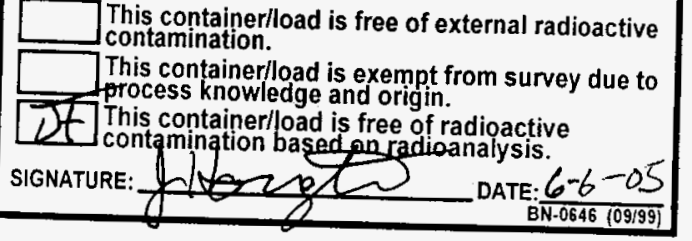


Driver rije 570 ele/aed

Truek: Kest-0709A

Trailer: Elo4326

$3: 00$

Bechtel Nevada NTS Landfill Load Verification

(Waste definitions are available on page 2)

$\begin{array}{lllll}\text { SWO USE (Circle One Area) AREA } & 23 & 6 & 9 & \text { LANDFILL }\end{array}$

For waste characterization, approval, and/or assistance, contact Solid Waste Operation (SWO) at 5-7898.

$$
\text { REQUIRED: WASTE GENERATOR INFORMATION }
$$

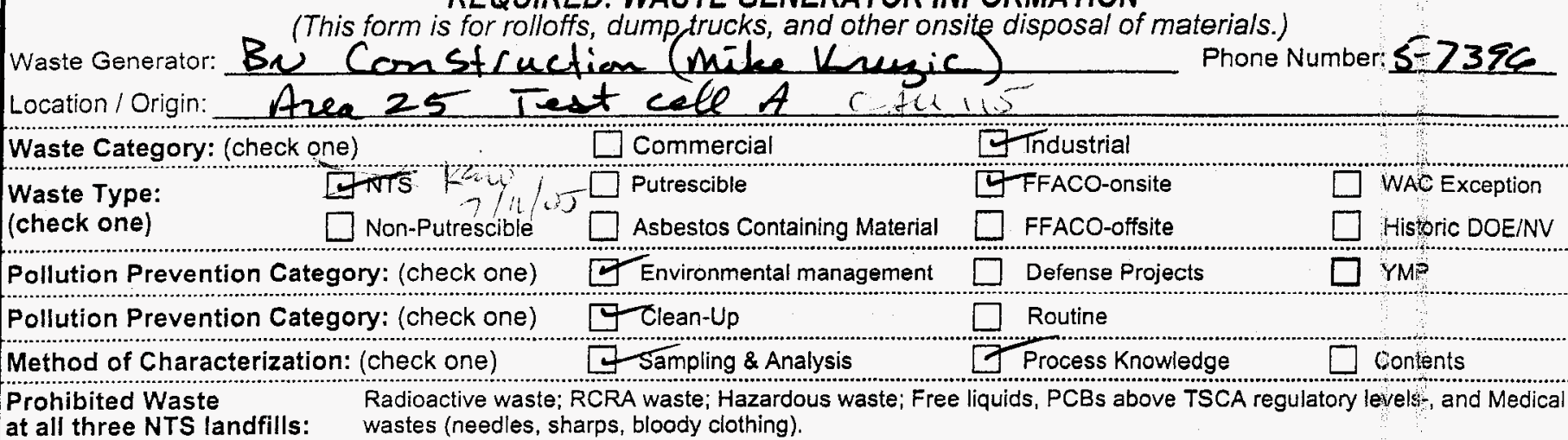

Additional Prohibited Waste Sewage Sludge; Animal carcasses-, Wet garbage (food waste); and Friable asbestos at the Area 9 U10c Landfill:

REQUIRED: WASTE CONTENTS ALLOWABLE WASTES

Check all allowable wastes that are contained within this load:

NOTE: Waste disposed at the Area 6 Hydrocarbon Landfill must have come into contact with petroleum hydrocarbons or coolants stich as:

gasoline (no benzene, lead); jet fuel; diesel fuel: lubricants and hydraulics; kerosene; asphaltic petroleum hydrocarbon; and ethylene glycol.

Acceptable waste at any NTS landfill: $\quad \square$ Paper $\quad \square$ Rocks / unaltered geologic materials $\square$ Empty containers
$\square$ Asphalt
$\exists$ Metal
$\square$ Wood
Soil
$\square$ Rubber (excluding tires)
$\square$ Plastic
7 Wire
$\square$ Cable
Cloth
$\square$ Insulation (non-Asbestosform)
GDemolion debris
GCemerit \& concrete

— Manufactured items: (swamp coolers, furniture, rugs, carpet, electronic components, PPE, etc.)

Additional waste accepted at the Area 23 Mercury Landfill: $\square$ Office waste $\square$ Food Waste $\square$ Animal Carcasses

Asbestos: $\square$ Friable $\square$ Non-Friable (contact SWO if regulated load) Quantity:

Additional waste accepted at the Area 9 U10c Landfill:

$\begin{array}{ll}\square \text { Non-friable asbestos } & \square \text { Drained automobiles and military vehicles } \\ \square \text { Light ballasts (contact SWO) } \square \text { Drained fuel filters (gas \& diesel) } \\ \square \text { Hydrocarbons (contact SWO) } \square \text { Other }\end{array}$

Additional waste accepted at the Area 6 Hydrocarbon Landfill:

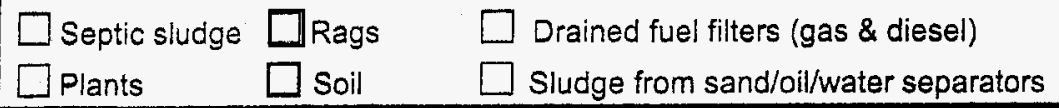
REQUIRED: WASTE GENERATOR SIGNATURE

Initials: (If initialed, no radiological clearance is necessary.)

The above mentioned waste was generated outside of a Controlled Waste Manag knowledge, does not contain radiological materials.

To the best of my knowledge, the waste described above contains only those ma site. I have verified this through the waste characterization method identified abc prohibited and allowable waste items.

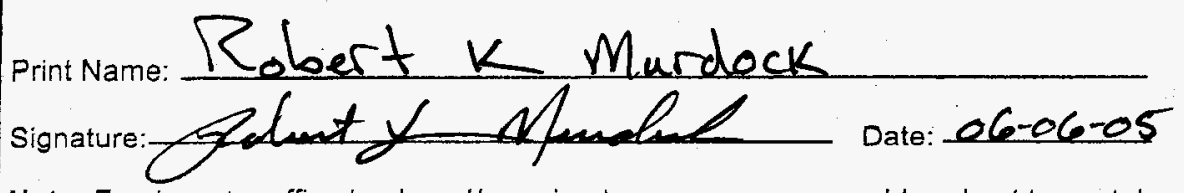

Solid fractions from sand/oil/water separators

Deconned Underground and Above Ground Tanks

Other

Crushed non-terne plated oil filters

PCBs below 50 parts per millon.

Note: Food waste, office trash and/or animal carcasses are considered not to contain added radioactivity, and therefore do not require a radiological clearance.

\section{SWO USE ONLY}

Load Weight (net from scale or estimate): 02,402

Signature of Certifier:

Radiation Survey Release for Waste Disposal RCT Initials

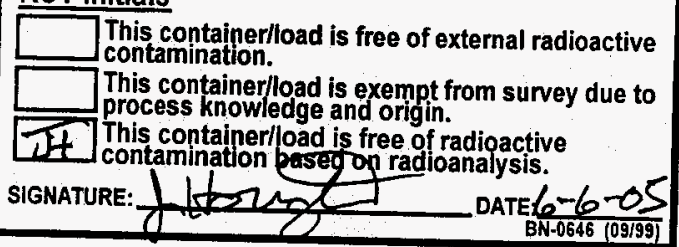



Driver: Arria/A $Z$ Tht7nAs
Patersiof

Truck: $G 82-07314$

Traila: Elo4817

\section{Bechtel Nevada NTS Landfill Load Verification}

(Waste definitions are available on page 2)
SWO USE (Circle One Area) AREA
23
6
9
LANDFILL

For waste characterization, approval, and/or assistance, contact Solid Waste Operation (SWO) at 5-7898.

$$
\text { REQUIRED: WASTE GENERATOR INFORMATION }
$$

(This form is for rolloffs, dump trucks, and other onsite disposal of materials.)

Waste Generator: Bu construction (mile kumje)

Waste Category: (check one) $\square$ Commercial

Waste Type: $\quad \square$ TTSK $7 / 1 \%, \square$ Putrescible

(check one) $\square$ Non-Putrescible

Pollution Prevention Category: (check one)

Pollution Prevention Category: (check one)

Method of Characterization: (check one)

$\square$ Asbestos Containing Material

7 Environmental management

8 Člean-Up

7 Sampling \& Analysis
WAC Exception

7 FFACO-onsite

FFACO-offsite

Defense Projects

Routine

Process Knowledge

Content

at all three NTS landfills: wastes (needles, sharps, bloody clothing).

Additional Prohibited Waste Sewage Sludge; Animal carcasses-, Wet garbage (food waste); and Friable asbestos at the Area 9 U10c Landfill:

\section{REQUIRED: WASTE CONTENTS ALLOWABLE WASTES}

Check all allowable wastes that are contained within this load:

NOTE: Waste disposed at the Area 6 Hydrocarbon Landfill must have come into contact with petroleum hydrocarbons or coolants such as: gasoline (no benzene, lead); jet fuel; diesel fuel; lubricants and hydraulics; kerosene; asphaltic petroleum hydrocarbon; and ethysene glycol.

Acceptable waste at any NTS landfill: $\square$ Paper $\square$ Rocks / unaltered geologic materials $\square$ Emptyicontainers
$\square$ Asphalt
$\square$ Metal
$\square$ Wood
Soil
$\square$ Rubber (excluding tires)
$\square$ Plastic
7 Wire
$\square$ Cable $\square$ Cloth
$\square$.nsulation (non-Asbestosform)
7 Demolition debris

$\square$ Manufactured items: (swamp coolers, furniture, rugs, carpet, electronic components, PPE, etc.)

Additional waste accepted at the Area 23 Mercury Landfill: $\square$ Office waste $\square$ Food Waste $\square$ Animal Carcasses

$\square$ Asbestos: $\square$ Friable $\square$ Non-Friable (contact $S W O$ if regulated load) Quantity:

Additional waste accepted at the Area 9 U10c Landfill:

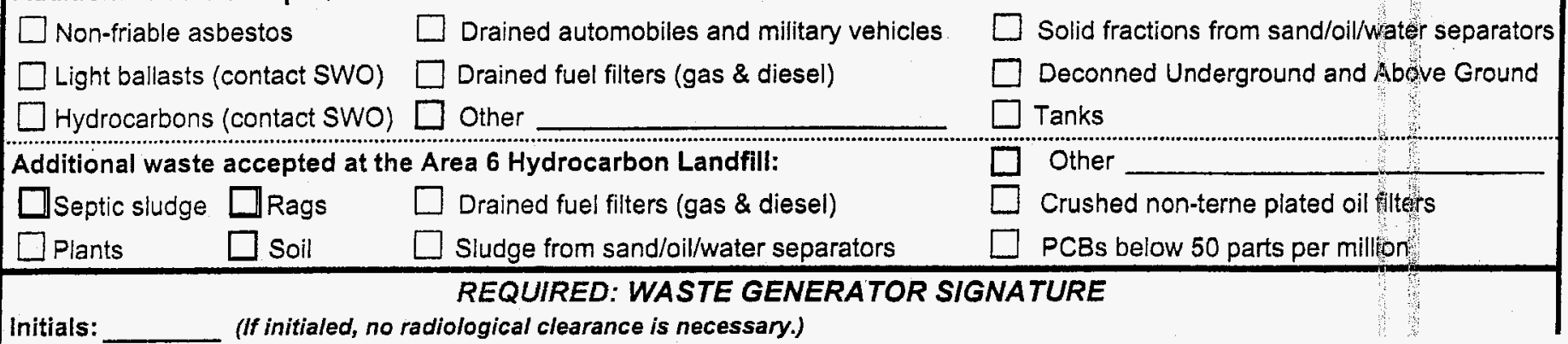

The above mentioned waste was generated outside of a Controlled Waste $M$ knowledge, does not contain radiological materials.

To the best of my knowledge, the waste described above contains only thos site. I have verified this through the waste characterization method identifiec prohibited and allowable waste items.

Print Name: Rabert k Murdock

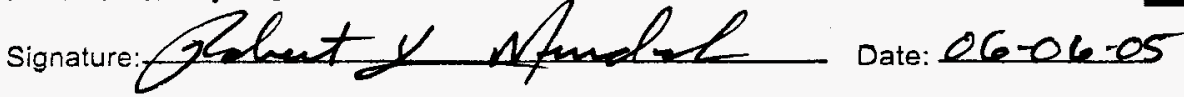

Radiation Survey Release for Waste Disposal

RCT Initials contamination.

This container/load is exempt from survey due to This containerlload is exempt fiom
process knowledge and origin.

TH This container/load is free of radioactive

contamipation based fon radioanalysis.

SIGNATURE:

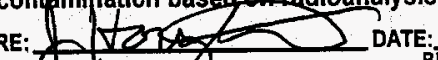

Note: Food waste, office trash and/or animal carcasses are considered not to contain added radioactivity, and therefore do not reguire a radiological clearance.

SWO USE ONLY

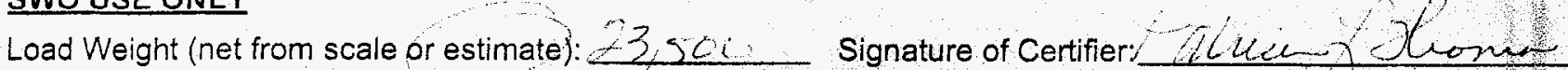


Driver A Aphis:

Truck: $682-0699 \mathrm{~A}$

\section{Bechtel Nevada}

\section{NTS Landfill Load Verification}

(Waste definitions are available on page 2)

\section{SWO USE (Circle One Area) AREA \\ 23 \\ 6 \\ 9. LANDFILL}

For waste characterization, approval, and/or assistance, contact Solid Waste Operation (SWO) at 5 -7898.

\section{REQUIRED: WASTE GENERATOR INFORMATION}

(This form is for rolloffs, dump trucks, and other onsite disposal of materials.)

Waste Generator: BU Construction (mike Kunic)

Location/Origin: Area 25 Teat cell A $6 \mathrm{dil}$

Waste Category: (check one)

Waste Type:

NTS

$\square$ Commercial
$\square$ Putrescible
$\square$ Asbestos Containing Material
$\square$ Environmental management
$\square$ Clean-Up
$\square$ Sampling \& Analysis

\begin{tabular}{l}
\hline Industrial \\
$\square$ FFACO-onsite \\
$\square$ FFACO-offsite \\
$\square$ Defense Projects \\
$\square$ Routine \\
$\square$ Proctoric DOE/NV \\
\hline Process Knowledge
\end{tabular}

(check one)

Non-Putrescible

- Sampling \& Analysis

Phone Number: $5-7396$

Pollution Prevention Category: (check one)

Pollution Prevention Category: (check one)

Method of Characterization: (check one)

Radioactive waste; RCRA waste; Hazardous waste; Free liquids, PCBs above TSCA regulatory levels-, and Medical

Prohibited Waste

wastes (needles, sharps, bloody clothing).

Additional Prohibited Waste Sewage Sludge; Animal carcasses-, Wet garbage (food waste); and Friable asbestos at the Area 9 U10c Landfill:

\section{REQUIRED: WASTE CONTENTS ALLOWABLE WASTES}

Check all allowable wastes that are contained within this load:

NOTE: Waste disposed at the Area 6 Hydrocarbon Landfill must have come into contact with petroleum hydrocarbons or coolants sich as: gasoline (no benzene, lead); jet fuel: diesel fuel: lubricants and hydraulics; kerosene; asphaltic petroleum hydrocarbon; and ehy hene glycol

Acceptable waste at any NTS landfill: $\square$ Paper $\square$ Rocks / unaltered geologic materials $\square$ Empty containers

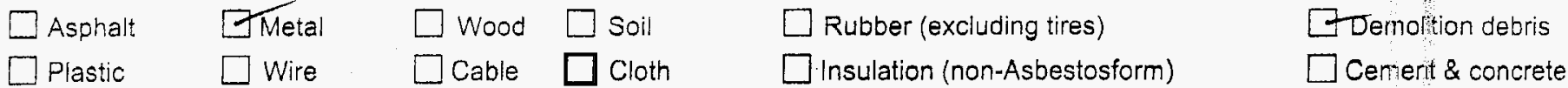

$\square$ Manufactured items: (swamp coolers, furniture, rugs, carpet, electronic components, PPE, etc.)

Additional waste accepted at the Area 23 Mercury Landfill: $\square$ Office waste $\square$ Food Waste $\square$ Animal Carcasses

$\square$ Asbestos: $\square$ friable $\square$ Non-Friable (contact SWO if regulated load) Quantity:

Additional waste accepted at the Area 9 U10c Landfill:

$\square$ Non-friable asbestos
$\square$ Light ballasts (contact SWO) $\square$ Drained fuel filters (gas \& diesel)
$\square$ Hydrocarbons (contact SWO) $\square$ Other

$\square$ Solid fractions from sand/oil/water separators

Additional waste accepted at the Area 6 Hydrocarbon Landfill:

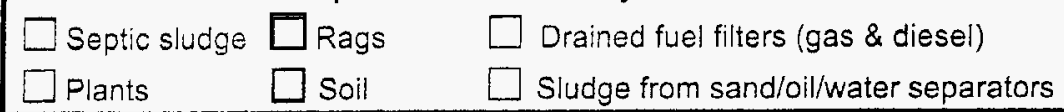
REQUIRED: WASTE GENERATOR SIGNATURE

Initials: (If initialed, no radiological clearance is necessary.)

The above mentioned waste was generated outside of a Controlled Waste knowledge, does not contain radiological materials.

To the best of my knowledge, the waste described above contains only th site. I have verified this through the waste characterization method identil prohibited and allowable waste items.

Print Name: Robent K Murdock $\square$ Deconned Underground and Above Ground $\square$ Tanks

$\square$ Other

$\square$ Crushed non-terne plated oil illters

\section{$\square$ PCBs below 50 parts per million}

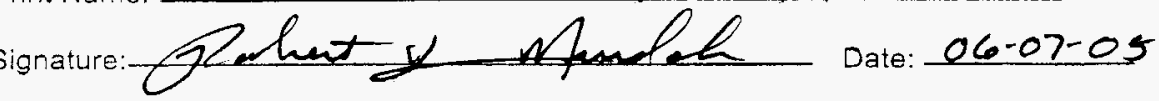

Note: Food waste, office trash and/or animal carcasses are considered not to contain added radioactivity, and therefore do not require a radiological clearance.

SWO USE ONLY

Load Weight (net from scale of estimate):

Radiation Survey Release for Waste Disposal RCT Initials

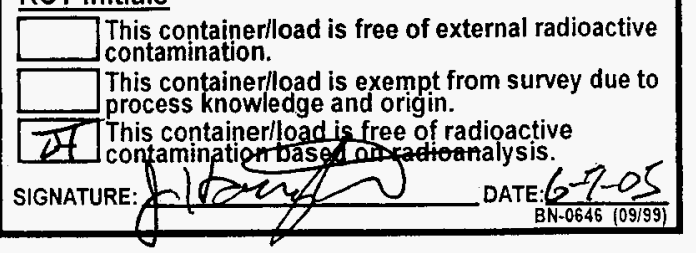


Driver

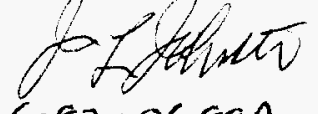

Truck:

$682-0688 \mathrm{~A}$

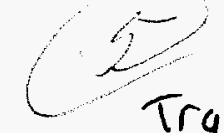

Trailer: E1000 35

9130

\section{Bechtel Nevada NTS Landfill Load Verification}

(Waste clefinitions are available on page 2)

\begin{tabular}{llllll}
\hline SWO USE (Circle One Area) AREA & 23 & 6 & (9) LANDFILL
\end{tabular}

For waste characterizetion, approval, and/or assistance, contact Solld Waste Operation (\$WO) at 5-7893.

REQUIRED: INASTE GENERATOR INFORMATION

(This form is for rolloffs, dump trucks, and other onsite disposal of materials.)

Waste Generator: BNCanstruetion (milh Vuchic

Waste Category: (check one)

Waste Type:

(check ons)

$\square$ Commerclal
$\square$ Putrescible
$\square$ Asbestoe Containing Materta!

\section{TIndustrial}

7 FFACO-Onslte

Phone Number: $5-2376$

Pollution Provontion Category: (check one)

Method of Characterization: (check one)

7 Environmental management

$\square$ FFACD-Dffsite

D Elean-Up

7 Sampling \& Analysis

$\square$ Defenze Projects

$\square$ Routine

Prohibited Wasto

Radloactive waste; RCRA waste; Hazandous waste; Free liquids, PCBs above TSCA regutatory levels". and Medical

at all three NTS landfills:

wastes (needies, sharps, bloody clothing).

Additional Prohibited Waste Sewage Sludge; Animal carcasses-, Wet garbage (food waste); and Frlable asbestos

at the Area 9 U10c Landfill:

REQUIRED: WASTE CONTENTS ALLOWABLE WASTES

Check all allowable wastes that are contained within this load

NOTE: Waste disposed at the Area 6 Hydrocarbon Landfill must have come into contact with patroleum hydnocarbons or coolants such as:

gasoline (no benzene, lead): jet fuel: desel fuel; lubrlcants and hydraulics kerosene; asphaltic pevoleum hydrocarbon; and ethylene glycol

Acceptable waste at any NTS landfill: $\square$ Paper $\square$ Rocks / wnaitered geologic materlals

$\square$ Asphalt $\quad$ Metal $\square$ wood $\square$ soil

$\square$ Rubber (excluding tires)

$\square$ Plastic

$\square$ Wire

$\square$ Cable $\square$ Cloth

पinsulation (non-Asbestosform)

Empty containers

Tomolition debris

$\square$ Manufactured items: (swamp coolers, furniture, rugs, carpet, electronic components, PPE, etc.)

Addlional waste accepted at the Area 23 Mercury Landfill: $\square$ Offico waste $\square$ Food Weste $\square$ Animal Careasses

$\square$ Asbestos: $\square$ Friable $\square$ Non-Friable (contact SWO lf regulated load) Quantity

Additional was te aceepted at the Area 9 U10c Landfill:

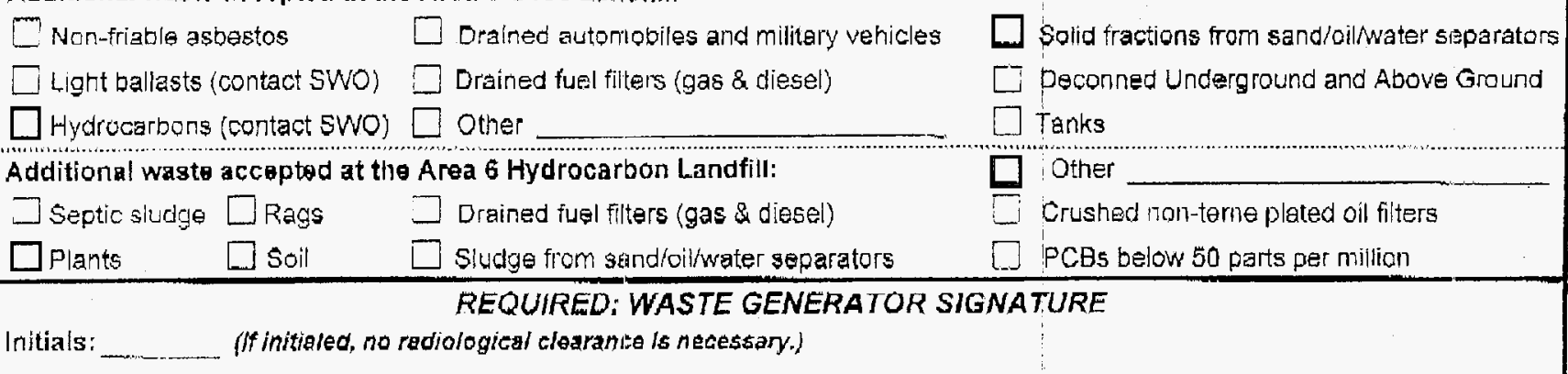

The above mentioned waste was generated outsicie of a Controlled Waste Manag

knowledge, does not contaln rediological materiais.

To the best of my knowledge, the waste descrlbed above contains only those ma site. I have verified this through the waste characrerlzation method identified abc prohlbited and allowabie waste items.

Print Name: Robert $k$ Murdock

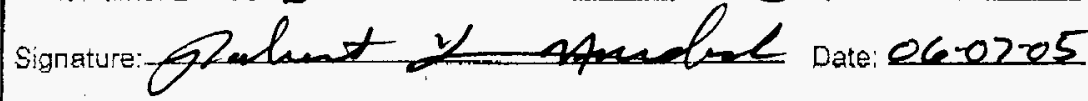

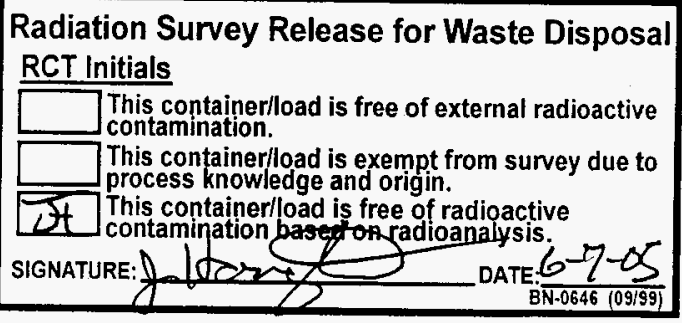

Note: Food waste, office tragh andior animal carcasses are considered not to contain added radioactivity, and therefore do net raquira a radiological clearance.

SWO USE ONLY

Load Weight (net from scale or estimate): $2 / .520$

Signature of Certifier: 


\section{Driver Jim S10\%lated}

Fruck G82-02094

Trailer: E 104326 PABE 3 or 3

\section{Bechtel Nevada NTS Landfill Load Verification}

(Waste clefinitions are available on page 2)

\section{SWO USE (Circle One Area) AREA \\ 23 \\ 6 \\ (9) \\ LANDFILL}

For wasto characterization, approva!, and/or assistance, contact Solld Waste Operation (SWO) at 5-7893.

$$
\text { REQUIRED: WASTE GENERATOR INFORMATION }
$$

(This form is for rolloffs, dump trucks, and other onsite disposal of materials.)

\begin{tabular}{|c|c|c|c|}
\hline Waste Category: (check one) & Commercial & \multicolumn{2}{|l|}{7 industrial } \\
\hline \multirow{2}{*}{$\begin{array}{l}\text { Waste Type: } \\
\text { (check one) }\end{array}$} & $\square$ Putrescible & 7 FFACO-Onslte & $\square$ WAC Excoption \\
\hline & $\square$ Asbestos Containing Material & $\square$ FFACO-dffsite & $\square$ HHstoric DOE/NV \\
\hline Pollutlon Prevention Category: (check one) & $\checkmark$ Environmental management & $\square$ Dafenae Projects & $\square$ YMP \\
\hline Pollution Provention Category: (check one) & 7 Clean-Up & $\square$ Routne & \\
\hline \multicolumn{2}{|l|}{ Method of Characterization: (check one) } & 7 Frocess Knowledge & Contents \\
\hline
\end{tabular}

Prohibited Wasto Radleactive waste; RCRA waste; Hazandous waste; Free liquids, PCBs above TSCA regulatory levels", and Medical

at all three NTS landfills: wastes (needles; sharps, bloody clothing).

Additional Prohibited Waste Sewage Sludge; Animal carcasses-, Wat garbage (food waste); and Frlable asbeatos at the Area 9 U1Dc Landfill:

REQUIRED: WASTE CONTENTS ALLOWABLE WASTES

Check all allowable wastes that are contained within this load.

NOTE: Waste disposed at the Area 6 Hydrocarbon Landfill must have come into contact with petroleum hydrocarbons or coolants such as: gasoline (no benzene, lead); jet fuel: desel fuel; lubricants and hydraulics; kerosene; asphaltic patroleum hydrocarbor; and ethylene glycol. Acceptable waste at any NTS landfill: $\square$ Paper $\square$ Rocks / unaitered deologie materlals $\square$ Empty containers
$\square$ Asphalt
7 Metal
[D Wod
$\square$ Sail
$\square$ Rubper (excluding tires)
$\exists$ Tire
$\square$ Cable . $\square$ Cloth
$\square$ insulation (non-Asbestosform)
7 Cemolition debris
$\square$ Plastic

$\square$ Manufactured items: (swamp coders, furniture, rugs, carpet, electronic components, PPE, etc.)

Addltional waste accepted at the Area 23 Mercury Landfill: $\square$ Office waste $\square$ Foqd Waste $\square$ Animal Careasses

$\square$ Asbestos: $\square$ Friable $\square$ Non-Friable (contact swo lf regulated load) Quantity:

Additional waste accepted at the Area 9 U10c Landfill:

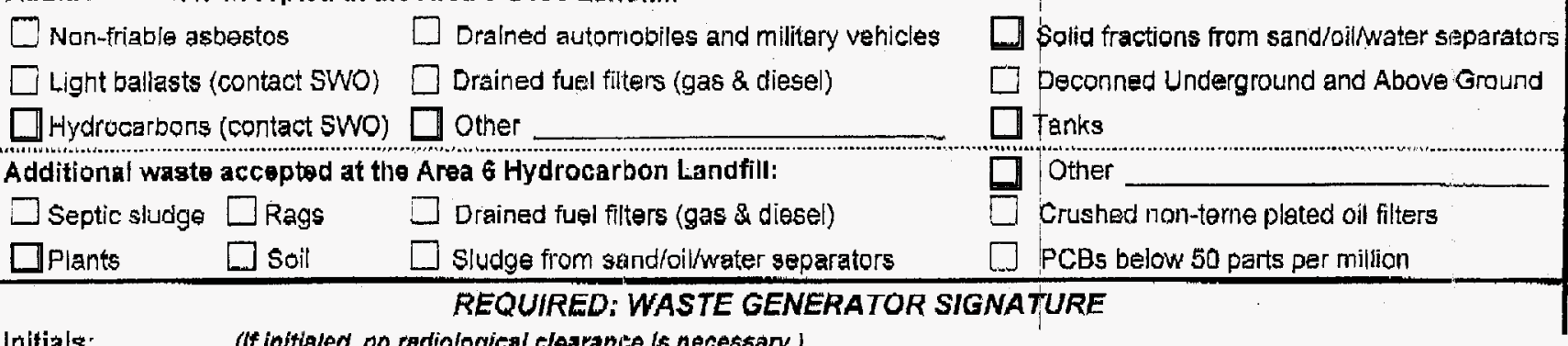

Initials: (If initialed, no radiological clearanie is necessary.)

The above mentioned waste was generated outsicle of a Controlled Was! knowledge, does not contaln radiological materials.

To the best of my knowledge, the waste described above contains only site. I hav verified this through the waste characierlzation method iden prohlbited and allowable wasto itoms.

Print Name Robert KMurdock

Radiation Survey Release for Waste Disposal RCT Initials

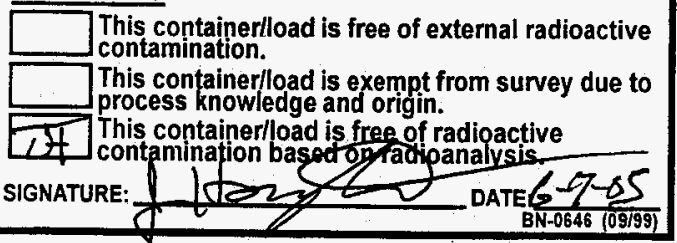

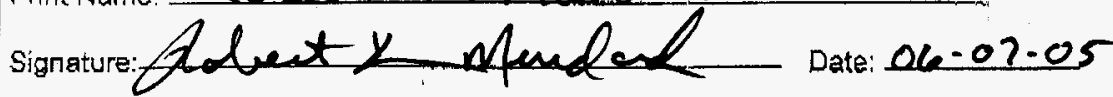

Note: Food waste, office trash and/or animal carcasses are considered not to contain added radioactivity, and therefore to not require a radiological clearance.

sWo USE ONLY
Load Weight (net from soale a estimate) $22(2)$ Signature of Certifier

r.

Hols 
Dsiver:

NTS Landfill Load Verification

(Waste clefinitions are avalable on page 2)

\begin{tabular}{|c|c|c|c|c|}
\hline SWO USE (Circle One Area) AREA & 23 & 6 & (9) & LANDFILL \\
\hline
\end{tabular}
REQUIRED: WASTE GENERATOR INFORMATION

(This form is for rolloffs, dump trugks, and other onsite disposal of materials.)

waste Generator: BN Construction (mil. Kunic) Phone Number: 5-7396 Location /Origin: Area 25 Test Cell A CAClls

Waste Category: (check one) $\quad \square$ commercla

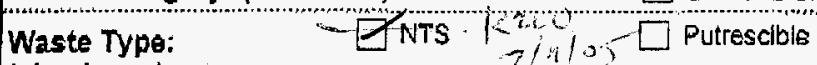

(check one)

$\square$ Nen-Putrescible

$\square$ Asbestos Containing Materia!

7 Industrial

Pollutlon Prevention Category: (check one)

Pollution Prevention Category: (check one)

7 Environmental management

4 FFACO-onsite

FACO-onsite

7 Clean-Up

Method of Characterization: (check one)

7 Sampling \& Analysis

$\square$ Defense Projects

$\square$ Rputine

Prohibited Waste $\quad$ Radloactive waste; RCRA waste; Hazandous waste; Frea liquids PCBs above TSCA

at all three NTS landfills: wastes (needles; sharps, bloody clothing).

Additional Prohibited Wagte Sevwage Sludge; Animal carcasses-, Wat garbage (food waste); and Frlable asbestos

at the Area 9 U10c Landffll:

REQUIRED: WASTE CONTENTS ALLOWABLE WASTES

Check all allowable wastes that are contained within this load:

NOTE: Waste disposed at the Area 6 Hytrosarbon Landfill must have come Into contact with petroleun hydrocarbons or coolants such as:

........... gsoline (no benzene, lead); jet fuel: dlesel fuel; lubrloants and hydraulics; kerosene; asphaltic potroleum hydrocorbor; and ethylene glycol.

Acceptable waste at any NTS landfill: $\square$ Piaper $\square$ Rocks / unaitered geologic materlals

$\square$ Asphalt $\quad \square$ Metal $\quad \square$ Wood $\square$ soll $\square$ Rubber (excluding tires)

$\square$ Plastic $\quad$ Wwire $\quad \square$ Cable $\square$ Cloth $\square$ Insulation (nom-Asbestosform)

$\square$ Manufactured items; (swamp coolers, furniture, rugs, carpet, electronic components, PPE, etc.)

Additional waste accepted at the Area 23 Mercury Landill: $\square$ Office waste $\square$ Food Waste $\square$ Animal Careasses

$\square$ Asbestos: $\square$ Friable $\square$ Non-Friable (contact swo lf regulated load) Quantity:

Additional waste accepted at the Area 9 U10c Landill:

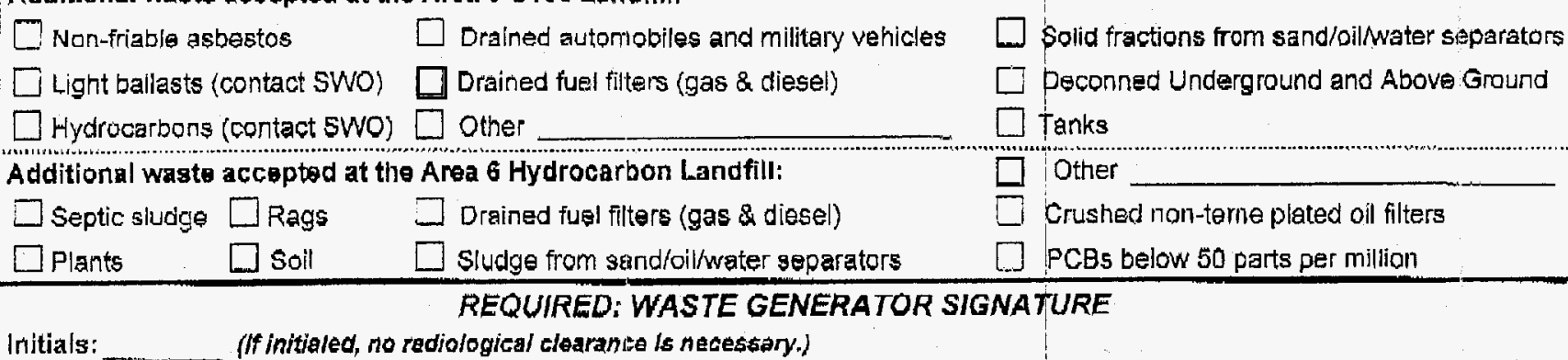

The above mentioned waste was generated outsicle of a Controlled Wasta Mana knowledge, does not contaln rediological materials.

To the best of my knowledge, the waste described above contains only thase $m$ site. I have verifiod this through the waste characterlzation method identified at prohlblted and allowable waste items.

Print Name: Robert $k$ Murdock

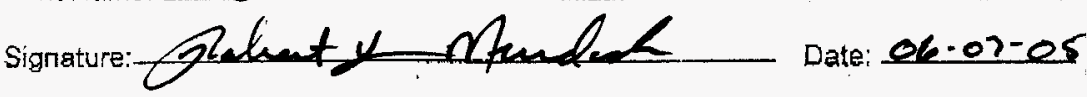

Radiation Survey Release for Waste Disposal RCT Initials

$\square$ This container/load is free of external radioactive contamination.

This container/load is exempt from survey due to process knowledge and origin.

य This containerlload is free of radioactive

SIGNATURE: 1 tor

Note: Food waste, office trash and/or animal carcasses are considered not to contain added radioactivity, and therefore do not raquire a radiological clearince.

SWO USE ONLY

Load Weight (net from scale or estima): 2950

Signature of Certifier 


\begin{tabular}{llllll}
\hline SWO USE (Circle One Area) AREA & 23 & 6 & (9) LANDFILL
\end{tabular}

For waste characterization, approva!, and/or assistance, contact Solid Waste Operation (SWO) at 5-7893.

REQUIRED: WASTE GENERATOR INFORMATION
This form is for rolloffs, dimp trucks, apd other onsite disposal of ma

Waste Generator: Location / Origin: ON Construction

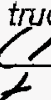

Waste Category: (check one) Area 25 net mike heric)

(check one)

$\square$ Commerclal

$47 n d u s t r i a l$

Pollutlon Prevention Category: (check one)

Pollution Prevention Category: (check one)

Method of Charactorizatjon: (chock one)

$\square$ Putrescible

2 FPACO-onslte

$\square$ Asbeslos Containing Materlal

$\square$ FffACD-offsite

C. Environmental management

$\square$ Clean-Up

7 Sampling \& Analysis

$\square$ Defense Projects

$\square$ Routlne

Prohibited Waste

Radloactive waste; RCRA waste; Hazandous

4 Process Knowledge

Phone Number: $5-7396$

at all three NTS landfills: wastes (needles, shorps, bloody clothing).

Additional Prohibitad Waste Sewage Sludge; Animal carcasses-, Wat garbage (food waste); and Frlable asbeatlos at the Area 9 U10c Landfill:

\section{REQUIRED: WASTE CONTENTS ALLOWABLE WASTES}

Check all allowable wastes that are contained within this toad:

NOTE: Waste disposed at the Area 6 Hydrocarbon Landfill must have come into contact with petroleum hydrocarbons or coolanis such as: gasoline (no benzene, lead); jet fuel: dlesel fuel; lubrlcants and hydraulics; kerosene; asphaltic potroteum hydrocarbon; and ethylene glycol

Acceptable waste at any NTS landfill: $\square$ Fiper $\square$ Rocks / unaitered deologic materlals

$\square$ Asphalt $\square$ Metal $\square$ wood $\square$ soil

$\square$ Plastic

$\square$ Wire

$\square$ Cable $\square$ Cloth

$\square$ Rubber (excluding tires)

$\square$ Insulation (non-Asbestosform)

$\square$ Empty containers (1) Demolition debris

$\square$ Manufactured items: (swamp coolers, furniture, rugs, carpet, electronic components, PPE, etc.)

Additional waste accepted at the Area 23 Mencury Landfill: $\square$ Office waste $\square$ Food Waste $\square$ Animal Cancasses $\square$ Asbestos: $\square$ Friable $\square$ Nor.Friable (contact $s$ Wo If regulated load) Quantity:

Additional waste accepted at the Area 9 U10c Landfill:

Non-friable asbestos $\quad \square$ Drained automobiles and military vehicles

$\square$ Light ballasts (contact SWO) $\square$ Drained fuel filters (gas \& diesel)

$\square$ Hydrocarbons (contact 5WO) $\square$ Other

Additional waste accepted at the Area 6 Hydrocarbon Landfill:

$\square$ septic sludge $\square$ Rags $\square$ Drained fuel filters (gas \& diesel)

$\square$ Plants $\quad \square$ soil $\square$ sludge from sund/oil/water geparators REQUIRED: WASTE GENERATOR SIGNATURE

Initials: (If initialed, no radiological clearanive is necessory.)

The above mentioned waste was generated outsicle of a Controlled Wasta 1 knowledge, does not contain rediological materials.

To the best of my knowledge, the waste descrlbed above contains only tha site. I have verified this through the waste characterlzation method identiffe prohlblted and allowable waste itams.

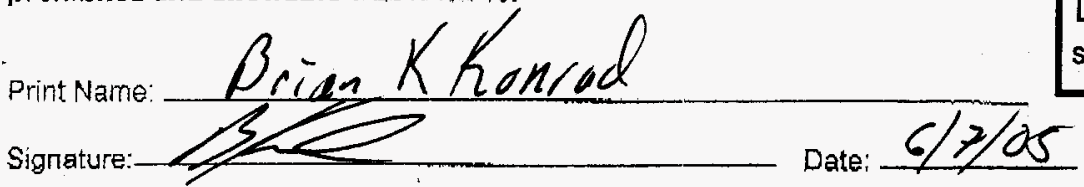

Radiation Survey Release for Waste Disposal RCT Initials

This container/load is free of external radioactive contamination.

This container/load is exempt from survey due to process knowledge and origin.

It This containerlload is free of radioactive Lt contamination based on radioanatysis. SIGNATURE: : Lation:

Note: Food waste, office trash and/or animal carcasses are considered not to contain added radioactivity, and therefore do mot require a radiological clearance.

SWO USE ONLY
Lozd Weight (net from scale of estimate) $201000 \quad 1500$ TB 


\section{NTS Landfill Load Verification Waste clefinitions are avallable on page 2! \\ Bechtel Nevada \\ 23 \\ 6 \\ 9 LANDF \\ SWO USE (Circle One Area) AREA}

For waste characterization, approva!, and/or assistance, contact Solld Waste Operation (\$WO) at 5-7898.

REQUIRED: WASTE GENERATOR INFORMATION

(This form is for rolloffs, dump trucks, and other onsite disposal of materials.)

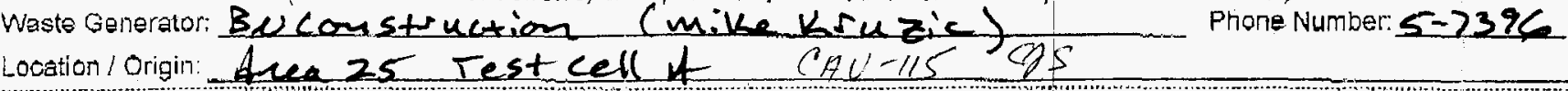

Waste Catagory: (check one)

Waste Type:

$\square$ commerclal

7 Thidustrial

Waste Typo:
(check one)

$\exists$ ITS

$\square$ Putrescible

TFACO-Onslte

$\square$ Non-Putrescible $\square$ Asbestos Containing Material

Pollution Prevention Category: (check one) oK $=$ Envmomentmagement

Pollution Provention Catsgory: (check one)

Method of Characterization: (check one)

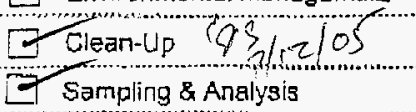

$\square$ FFACD-offsite

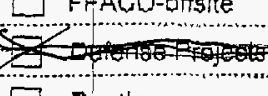

Routlne

Process knowledge

WAC Exception

HIstorle DOENV ans YMP

Prohibited Wagt

Radloactive waste; RCRA waste; Hazandous waste; Free liquids, PCBs above TSCA regulatory levels", and Medical at all throe NTS landfills:

wastes (needles; sharps, bloody clathing).

Additional Prohibited Waste Sewage Sludge; Animal carcasses-, Wet garbage (food waste); and Friable asbestos at the Area 9 U $10 \mathrm{C}$ Landfill:

REQUIRED: WASTE CONTENTS ALLOWABLE WASTES

Check all allowable wastes that are contained within this load:

NOTE: Waste disposed at the Area 6 Hyerocarbon Landfill nust have come Into contact with petroleum hydrocarbons or coolants such as:

gasoline (no benzene, lead); jet fuel: dlesel fuel; lubrlcants and hydraulics kerosene; asphaltic pefoleum fydrocarton; and ethylene glycol.

Acceptable waste at any NTS landfill:

$\square$ Asphalt $\square$ Metal $\square$ Wood $\square$ Soil $\square$ Rubber (excluding tires)

GPlastic $\square$ wire $\square$ Cable $\square$ cloth $\square$ Insulation (non-Asbestosform)

Empty containers

$\square$ Demolition debris

$\square$ Manufactured items: (swamp coolers, furniture, rugs, carpet, electronic components, PPE, etc.)

Additional waste accepted at the A rea 23 Mencury Landfil: $\square$ Office waste $\square$ Food Waste $\square$ Animal Caneasses

$\square$ Asbastos: $\square$ Friable $\square$ Non-Friable (contact SWO If regulated load) Quantity:

Additional was te accepted at the Area 9 U10c Landfill:

Non-friable asbastos $\square$ Drained automiobiles and military vehicles
$\square$ Light baliasts (contact swo) $\square$ Drained fuel filters (gas \& diesel)
$\square$ Hydrucarbons (contact swo) $\square$ Other Perlite

De Solid fractions from sand/oil/water separators

$\square$ Deconned Underground and Above Ground

Additional waste accepted at the Area 6 Hydrocarbon Landfill:

\begin{tabular}{|c|c|c|}
\hline$\square$ septic sludige & $\square$ Rags & $\square$ Drained fuel filters (gas \& diesel) \\
\hline Q Plants & soil & $\square$ sludge from sand/oiliwater separators \\
\hline
\end{tabular}

$\square$ Tanks

$\square$ Other

$\square$ Crushed non-terne plated oil filters

(] PCBs below 50 parts per million

Initials: __ _ (ffinitialed, no rediological clearanise is necessary.)

The above mentioned waste was generated outsicle of a Controlled Waste Manag knowledge, does not contaln radiological matariais.

To the best of my knowledge, the waste described above contains oniy those ma site. I have verified this through the waste characterlzation method identified abe prohlblted and allowab/e waste items.
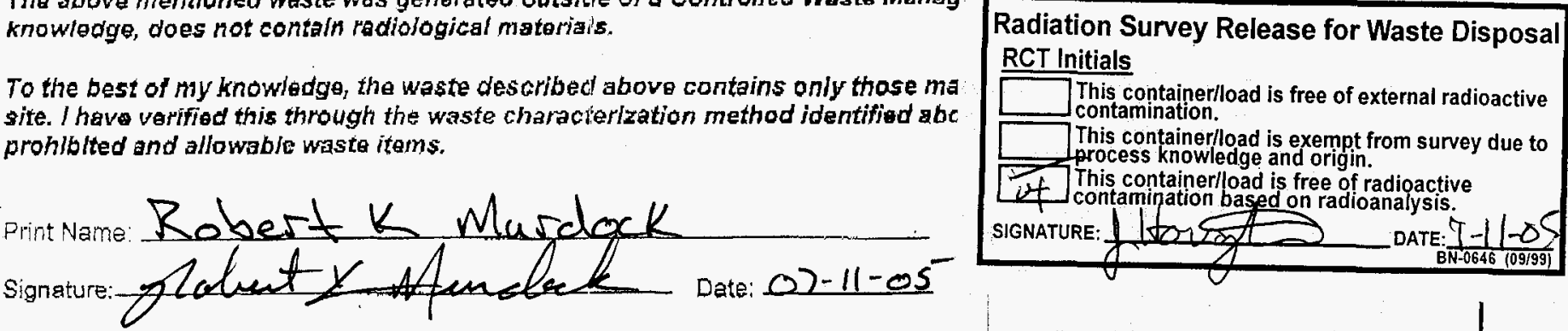

Note: Food waste, ofice trash andior a nimal carcasses are considered not to contrin added radioactivity, and therefore do ndt require a raciological clearance.

SWO USE ONLY

Load Weight (net from scale or estimate): 15000 Signature of Certifier 
For waste characterization, approval, and/or assistance, contact Solld Waste Operation (\$WO) at 5-7898.

$$
\text { REQUIRED: WNASTE GENERATOR INFORMATION }
$$

(This form is for rolloffs, dump trucks, and other onsite disposal of materials.)

\begin{tabular}{|c|c|c|c|c|}
\hline \multicolumn{5}{|c|}{ 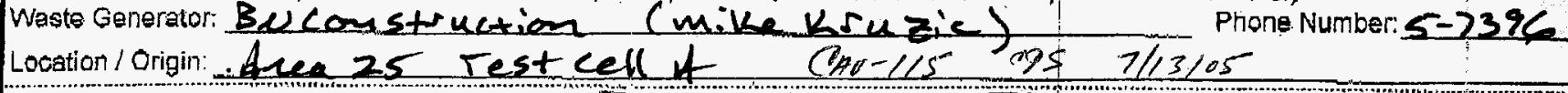 } \\
\hline \multicolumn{2}{|c|}{ Waste Category: (check one) } & $\square$ commerclal & \multicolumn{2}{|l|}{7 Thdustrial } \\
\hline \multirow{2}{*}{$\begin{array}{l}\text { Waste Type: } \\
\text { (check on } \theta \text { ) }\end{array}$} & YNTS & $\square$ Putrescible & GFFACO-Onsite & WAC Exception \\
\hline & $\square$ Non-Putrescible & $\square$ Asbestos Containing Matertal & $\square$ FfACO-Dfssite & Historle $\mathrm{DOE} / \mathrm{NV}$ \\
\hline \multicolumn{2}{|c|}{ Pollution Prevention Category: (check one) } & E Environmental management & $\square$ Defense Projects & $\square$ YMP \\
\hline \multicolumn{2}{|c|}{ Pollution Provention Category: (check one) } & $\Xi$ Cleari-Up & $\square$ Routhe & \\
\hline \multicolumn{2}{|c|}{ Method of Characterization: (check one) } & T Sampling \& A Aralysis & 7 Process knowledge & Contents \\
\hline
\end{tabular}

at all three NTS landfills: $\quad$ wastes (needles, sharpg, bloody clothing)

Additional Prohibited Waste Sewage Siudge; Aninal carcasses-, Wet garbage (food Waste); and Frlable asbeslos at the Area 9 U106 Landfill:

REQUIRED: WASTE CONTENTS ALLOWABLE WASTES

Check all allowable wastes that are containsd within this load.

NOTE: Waste disposed at the Area 8 Hyerocarbon Landfill must have come Into contact with petroleum hydrocarbons or coolants such as: gasbline (no benzene, lead); jet fuel: dlesel fuel; lubrlcants and hydraulics; kerosene; asphaltic pelroleum lydrocarbon; and ethylene glycol

Acceptable waste at any NTS landfill: $\square$ Paper $\square$ Rocks / wnaitered geologic materlals

$\square$ Asphalt $\square$ Metal $\square$ wood $\square$ soil $\square$ Rubber (excluding tires)

EPlastic $\square$ wire $\square$ Cable $\square$ Cloth

$\square$ Insulation (non-Asblestosform)

Empty containers

$\square$ Demolition debris

$\square$ Manufactured items: (swamp coolers, furniture, rugs, carpet, electronic components, pPE, atc.)

Additional waste accepted at the Area 23 Mercury Landfill: $\square$ Office waste $\square$ Food Waste $\square$ Animal Careasses

$\square$ Asbestos: $\square$ Friable $\square$ Non-Friable (contact SWO lf regulated load) Quantity:

Additional waste accepted at the Area 9 U10c Landfill:

Won-friabie asbestos $\quad \square$ Drahed autonobiles and militery vehicles

$\square$ Light ballasts (contact sWO) $\square$ Drined fuel filters (gas \& diesel)

D Hydrocarbons (contact SWO) POther Perlite

Additional waste accepted at the Area 6 Hydrocarbon Landfill:

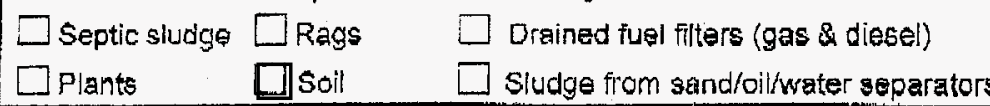

REQUIRED: WASTE GENERATOR SIGNATURE

Initials: (If initialed, no rediological clearanive is necessary.)

Tha abova mentioned waste was generated outsicle of a Controlled Waste Managenunt Area (CWMA) and to the best of my knowledge, does not contaln radiological materials.

To the best of my knowledge, the waste described above contains only those ma: site. I have varified this through the waste characterlzation method identified abo prohiblted and allowable waste items.
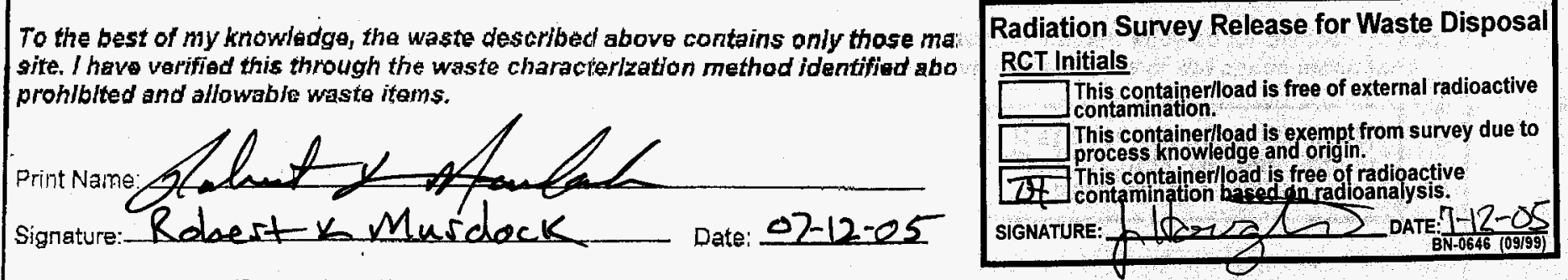

Note: Food waste, office trash and/or animal caroasses are considered not to contain abuci raviuactivicy, ano thererore eo not require a radiological clearance.

SWO USE ONLY

Load Weight (net from scale or

$$
\text { Roll off - } 009
$$
60.00

Solid fractions from sand/oil/water separator $[$ Deconned Underground and Above Ground $\square$ Tanks

[] Other

G Crushed non-terne plated oil filters

[] PCBs below 50 parts per millian

\section{Cemerst \& concroto}




\section{Bechtel Nevada NTS Landfill Load Verification} (Waste clefinitions are available on page 2.

\begin{tabular}{llllll}
\hline SWO USE (Circle One Area) AREA & 23 & 6 & 9 & LANDFILL
\end{tabular}

For waste characterization, approval, and/or assistance, contact Solld Waste Operation (SWO) at 5-7898.

REQUIRED: IWASTE GENERATOR INFORMATION

(This form is for rolloffs, dump trucks, and other onsite disposal of materials.)

Waste Generator: Bucoustuction (mike Kruzic) Phone Number:5-7396 Location / Origin: Area 25 Test cell \&

Waste Category: (check one)

CAU $1 / 15$ $\cos 1 / 20105$

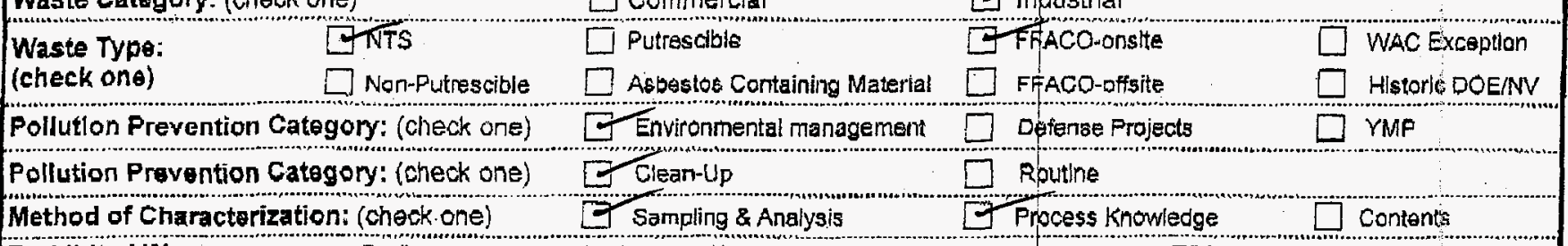

Prohibited Waste Radloactive waste; RCRA waste; Hazardous waste; Free liquids, PCBs above TSCA regulatory levels, and Medica! at all thrae NTS landfills: wastes (needles, sharps, bloody clathing).

Additional Prohibited Waste Sewage Sludge; Animal carcasses-, Wet garbage (food waste); and Frlable asbestos at the Area 9 U10e Landfill:

REQUIRED: WASTE CONTENTS ALLOWABLEIWASTES

Check all allowable wastes that are containod within this load:

NOTE: Waste disposed at the Area 6 Hycrocarbon Landfill must have come Into contzot with petroleum hydrocarbons or coolants such as: gesoline (no benzene, lead); jet fuel: dlesel fuel; lubrloants and hydraulics; kerosene; asphaltic patrolsum hydrocarbon; and ethylene glycol.

Acceptable waste at any NTS landfill: $\square$ Paper $\square$ Rocks / unaitered deologic materlals $\square$ Empty containers
$\square$ Asphat
$\square$ Metal
$\square$ wood
$\square$ Soil
$\square$ Rubper (excluding tires)
Dinsulation (nom-Asbestosform)
$\square$ Demolition debris
PFastio
$\square$ Wire
$\square$ Cable $\square$ Cloth

$\square$ Manufactured items: (swamp coolers, furniture, rugs, carpet, electronic components, ppe, etc.)
Additional wagte accepted at the Area 23 Mencury Landfill: $\square$ Office waste $\square$ Fopd Waste

Additional waste accepted at the Area 23 Mercury Landfill: $\square$ Office waste $\square$ Food Waste $\square$ Animal Careasses

$\square$ Asbestos: $\square$ Friable $\square$ Non-Friable (contact Swo if regulated load) Quantity:

Additional waste aceepted at the Area $\$$ UHOc Landfill:
Q Non-friable asbestos
$\square$ Drained autoriobiles and militery vehicles
Solid fractions from sand/oilwater separators
$\square$ Light ballasts (contact SWO) $\square$ Drained fuel filters (gas \& diesel)
Hydrocarbons (contact swo) Fother Perlite
Additional waste acceptod at the Area 6 Hydrocarbon Landfill:
$\square$ septic sludge
$\square$ Plants Rags $\quad \square$ Drained fuel filters (gas \& diesel)
$[$ Deconned Underground and Above Ground $\square$ Tanks
REQUIRED: WASTE GENERATOR SIGNATURE
Imitials:
(ff initialed, no radiological clearanite is nacestary.)

The above mentioned waste was generated outsicle of a Controlled Waste Management Area (CWMA) and to the best of my knowlodge, does not contaln rediological materiais.

To the best of my knowledge, the waste desaribed above contains only those site. I have verified this through the waste characlerization method identified prohlbited and allowable waste itams.
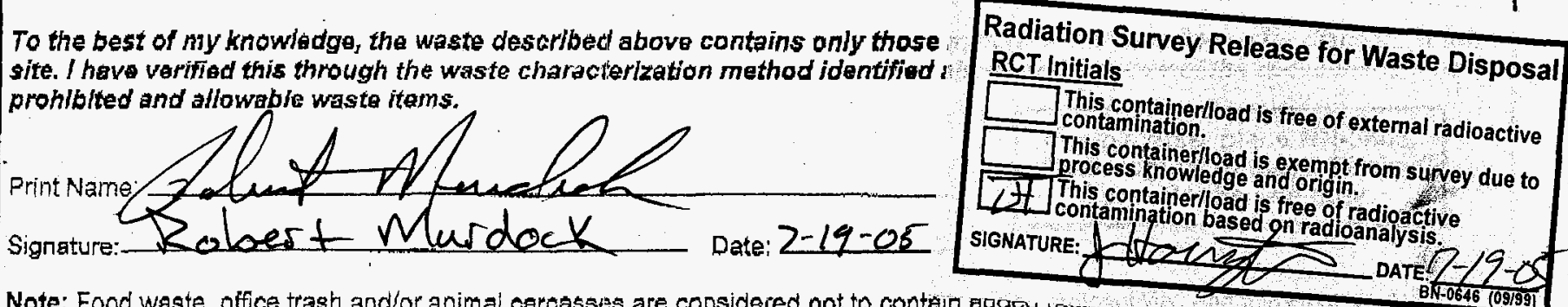

Note: Food waste, office trash and/or a nimal carcasses are considered not to conten angou ia... require a radiological clearance.

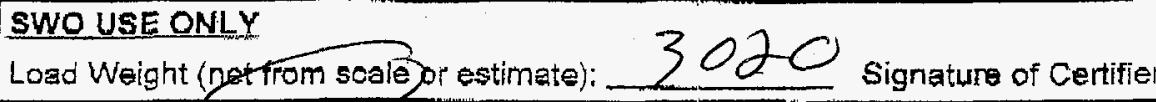

Traitar 00091120

Truck - E106400 
For waste characterization, approva!, and/or assistance, contact Solld Waste Operation (5WO) at 5-7898.

$$
\text { REQUIRED: WASTE GENERATOR INFORMATION }
$$

(This form is for rolloffs, dump trucks, and other onsite disposal of materials.)

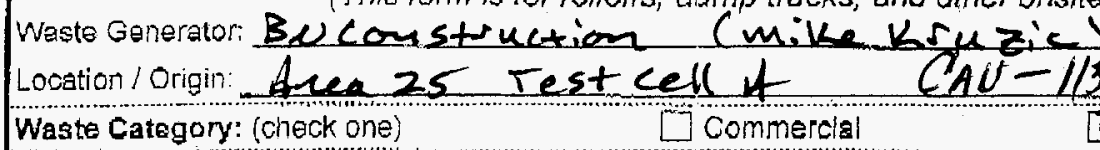

Waste Type:

7 NTS

(check one)

$\square$ Non-Putrescible

$\square$ Putrescible

Pollution Prevention Category: (check one)

Pollution Provention Category: (check one)

A Asbasloe Containing Material

Thdustria

Method of Characterization: (check one)
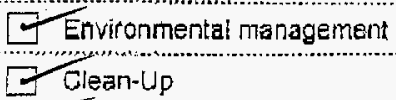

Prohibited Wasto

7 Sampling \& Analysis

Phone Number: $5-2396$

at all throe NTS landfills:

Process Knowledge

Radloaclive waste: RCRA waste; Hazando
wastes (needles: sharps, bloody clothing).

Additional Prohibitad Waste Sewage Sludge; Animal carcasses-, Wat garbage (food waste); and Friable asbestos at the Area 9 U10C Landfill:

\section{REQUIRED: WASTE CONTENTS ALLOWABLE WASTES}

Check all allowable wastes that are contained within this load:

NOTE: Waste disposed at the Area Hydrocarbon Landfill must have come into contact with petroleum hydrocarbons or coolants such as: gaspline (no benzene, lead); jet fuel; dlesel fuel; lubricants and hydraulics; kerosene; asphaltic petroleum hydrocarbon; and ethylene glycol

Acceptable waste at any NTS landfill: $\square$ Plaper $\square$ Rocks / unaltered geologic materlals $\square$ Empty containers
$\square$ Asphalt
$\square$ Metal
$\square$ wood
$\square$ Soil
$\square$ Rubber (excluding tires)
$\square$ Insulation (non-Asbestosform)
$\square$ Demolition debris
PFlastic
$\square$ Wire
$\square$ Cable $\square$ Ciloth
Cement $\&$ concrete

Z Manufactured items: (swamp coolers, furniture, rugs, carpet, electronic components, PPE, atc.)

Additional waste accepted at tho Area 23 Mercury Landill: $\square$ Ofice waste $\square$ Food Waste $\square$ Animal Careasses

$\square$ Asbestos: $\square$ Friable $\square$ Non-Friable (contact SWO If regulated load) Quantity:

Additional waste accepted at the Area $\$$ U10c Landfill:

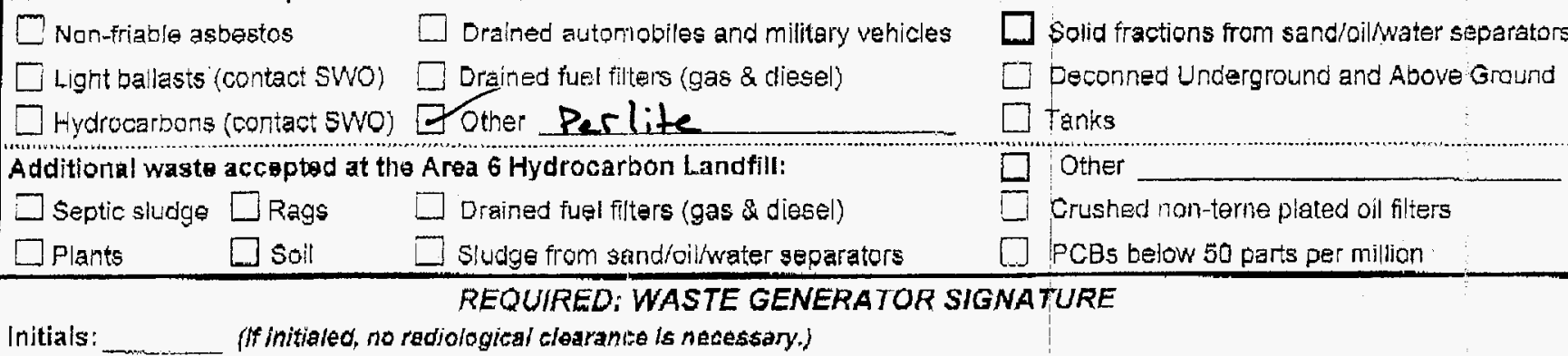

The above mentioned waste was generated outsicle of a Controlfed Waste Manage knowledge, does not contalt radiological materials.

To the best of my knowledge, the waste described above contains only thase mat site. I have verified this through the waste characierlzation method identified abov prohlbited and allowable waste items.
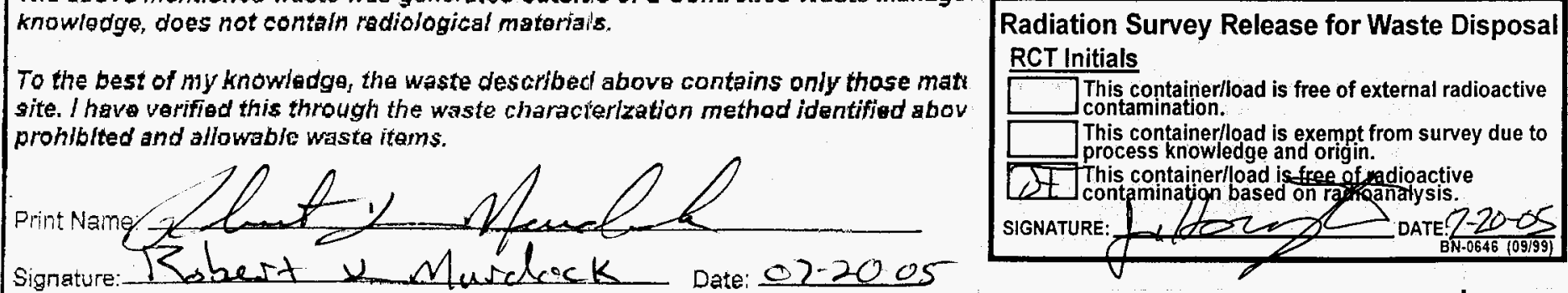

Note: Food waste, office trash and/or animal careassas are considered not to contain added radioactivity, and therefore do not require a radiological clearance.

SWO USE ONLW Load We ght (net from scale br estimate): 8280 signature of Certifier Truck $=E 106400$ (O) $4: 00$

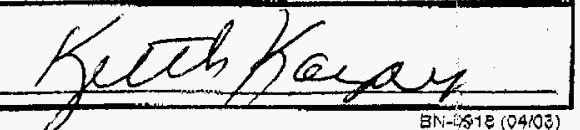

Trailer $=42$ 


\section{Bechtel Nevada \\ and}

NTS Landfill Load Verification

(Waste clefinitions are available on page 2)

\begin{tabular}{llllll}
\hline SWO USE (Circle One Area) AREA & 23 & 6 & (9) LANDFILL
\end{tabular}

For waste characterizetion, approva!, and/or assisfance, contact Solid Waste Operation (SWO) at 5-7898.

REQUIRED: INASTE GENERATOR INFORMATION

(This form is for rolloffs, diump trucks and other onsite disposal of materials.)

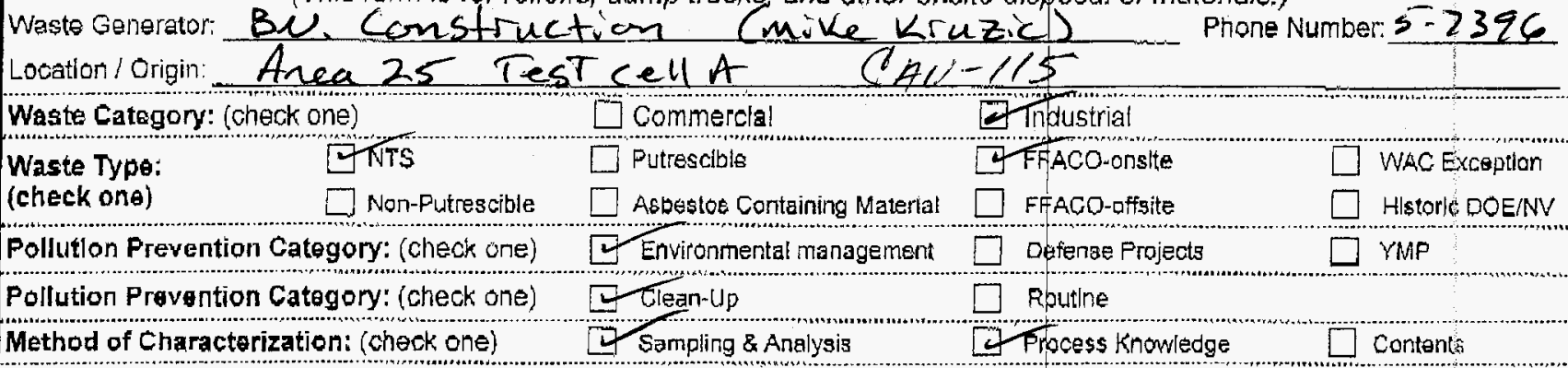

Prohibited Waste $\quad$ Radlaactive waste; RCRA waste; Hazandous waste; Free liquids, PCBs above TSCA regulatory levelsw, and Medical at all three NTS landfills: wastes (needies, sharps, bloody clothing).

Additional Prohibited Waste Sevage Sludge; Animal carcasses-, Wet garbage (food waste); and Frlable asbeslos at the Area 9 U10c Landfill:

\section{REQUIRED: WASTE CONTENTS ALLOWABLE WASTES}

Check all allowable wastes that are containod within this load

NOTE: Waste disposed at the Area 6 Hydrocarbon Landfill nust have come Into contact with petroleum hydnocarbons or coolants such as: gasbline (no benzene, lead); jet fuel: dlessl fuel; lutrescants and hydraulics; kerosene; asphattic petroloum hudrocarbon; and ethylene glycol

Acceptable waste at any NTS landfill: $\square$ Paper $\square$ Rocks / unaitered geologic materlals $\square$ Empty coltainers

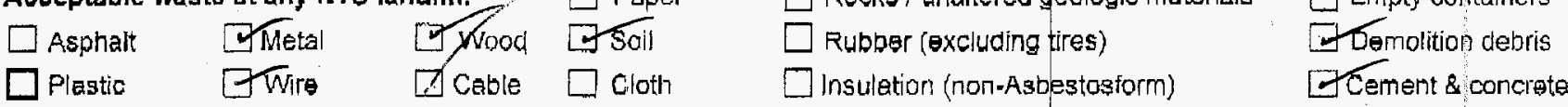

$\square$ Manufactured items: (swamp coolers, furniture, rugs, carpet, electronic components, PPE etc.)

Additional waste accepted at the Area 23 Mercury Landfill: $\square$ Office waste $\square$ Food Waste $\square$ Animal Careasces

$\square$ Asbestos: $\square$ Friable $\square$ Non-Friable (contact SWO lf regulated load) Quantity:

Additional waste accepted at the Area $\$$ U10c Landfill:

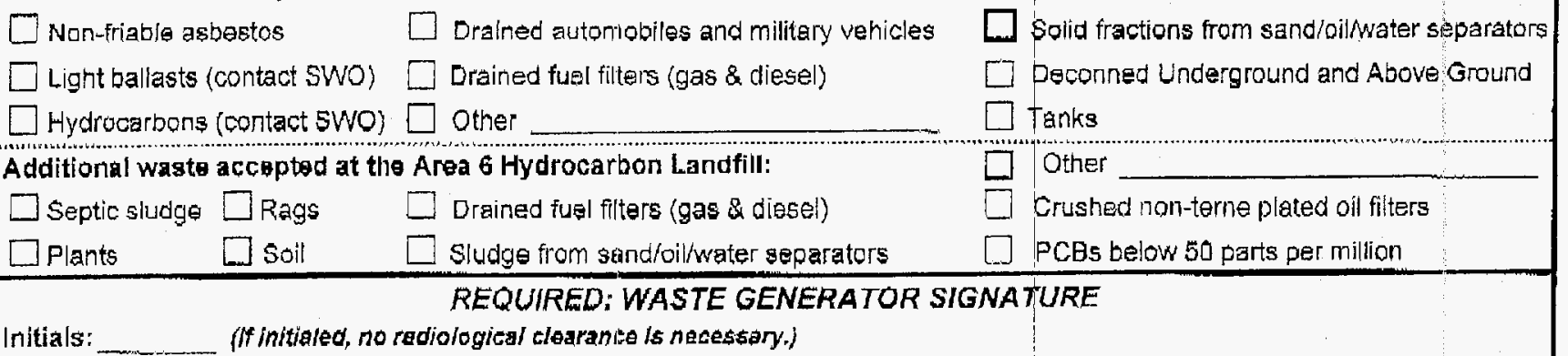

The above mentioned waste was generated outs/cle of a Controlled Waste Management Area (CWhA) and to the best of my knowledge, does not contaln radiological materia's.

To the best of my knowledge, the waste descrfbed above contains only site. I have verified this through the waste characterlzation method ider prohibited and allowabic waste items.

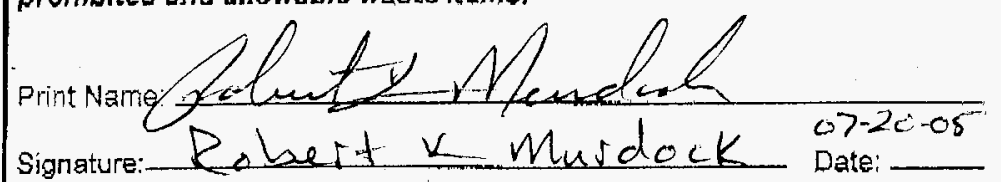

Note: Food waste, office tragh andior animal carcasses are considered not t require a radiological clearance.

SWO USE ONLY

Losd Weight (net from soaie (or estimate) 20,000 signatura of Certifier:

Trailer - E102925

(2) $11: 40$
Radiation Survey Release for Waste Disposal RCT Initials

This container/load is free of external radioactive contamination.

This container/load is exempt from survey due to process knowledge and origin.

This container/load is free of radioactive 21 contamination baseg on radioanalysis signature:

Truck - G820688A 


\section{Bechtel Nevada NTS Landfill Load Verification} (Waste clefinitions are availab/e on page 2)

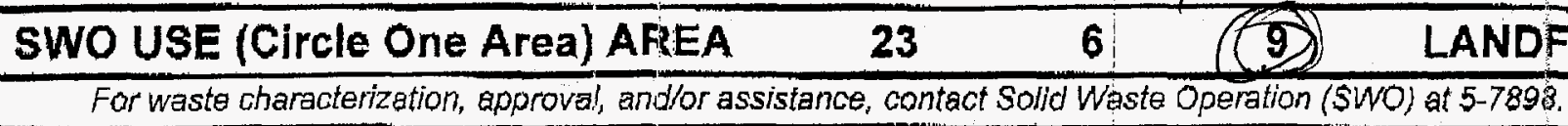
REQUIRED: WASTE GENERATOR INFORMATION

(This form is for rolloffs, dump trucks, and other onsite disposal of materials.)

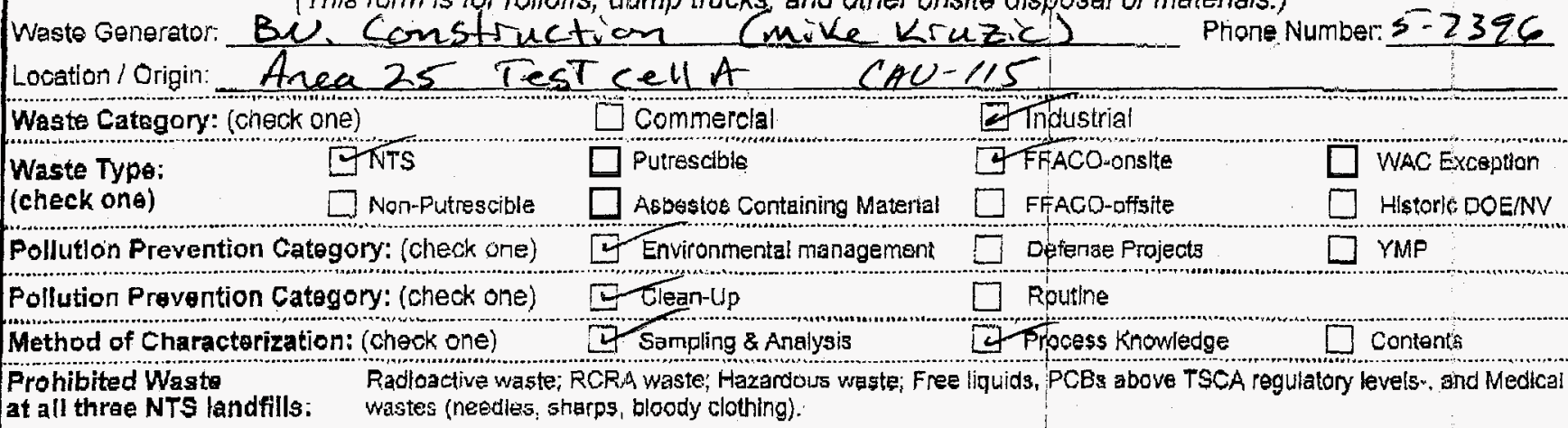
at all three NTS landfills: wastes (needles, sharps, bloody clothing).

Additional Prohibited Waste Sewage Sludge; Animal carcasses-, Wat garbage (food waste); and Frlable asbestos at the Area 9 U10c Landfill:

REQUIRED: WASTE CONTENTS ALLOWABLE WASTES

Check all allowable wastes that are contained within this load:

NOTE: Waste disposed at the Area Hydrocarbon Landfill nust have come Into contact with petroleum hydnocarbons or coolants such as: gasdine (no benzene, lead); jet fuel: dlesel fuel; lubrlcants and hydrautics; kerosene; asphaltic patroleum hydrocarbon; and ethylene glycol. Acceptable waste at any NTS landfill: $\square$ Paper $\square$ Rocks / whaltered geologic materlals $\square$ Emply containers

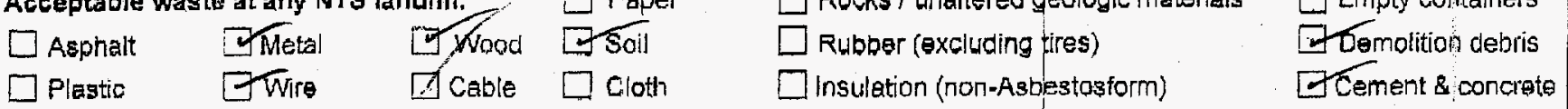

$\square$ Manufactured items: (swamp coolers, furniture, rugs, carpet, electronic components, PPE, etc.)

Additional waste accepted at the Area 23 Mercury Landfill: $\square$ Offico weste $\square$ Food Waste $\square$ Animal Careasses

$\square$ Asbestos: $\square$ Friable $\square$ Non-Friable (contact sWo if regulated load) Quantity:

Additional waste accepted at the Area $\$$ U 10c Landfill:

$\begin{array}{lll}\square \text { Non-friable asbestos } & \square \text { Drained automobiles and military vehicles } & \square \text { polid fractions from sand/oil/water saparators } \\ \square \text { Light ballasts (contact swo) } \square \text { Drained fuel filters (gas \& diesel) } & \square \text { peconned Underground and Above Ground } \\ \square \text { Hydrocarbons (contact SWO) } \square \text { Other } & \square \text { Tanks }\end{array}$ Additional waste acceptod at the Area 6 Hydrocarbon Landfill:
$\square$ septic siudge $\square$ Rags
$\square$ Drained fuel fitters (gas \& diesel)
$\square$ plants
Qu soil
$\square$ sludge from sandioil/water separators
E Crushed non-terne plated oil filters

$\square$ Other REQUIRED; WASTE GENERATOR SIGNATURE

Initials: (ff initialed, no rediological clearanica is necessary.)

The above mentioned waste was generated outsicle of a Controlfed Wast knowlodge, does not contaln radiological materiais.

To the best of my knowledge, the waste described above contains only th site. I have verified this through the waste characterization method identi prohlblted and allowabje waste items.

Print Name: Robert K Murdock

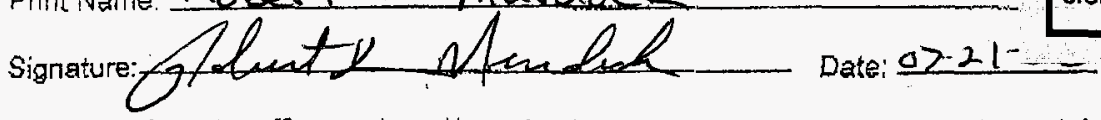

Radiation Survey Release for Waste Disposal RCT Initials

RCTInitials This containerloar

This containerlload is exempt from survey due to This containerload is exempt from
process knowledge and origin. process knowledge and orig in
This container/load is free of radioactive
contamination based on radioanalysis. SIGNATURE: RE: DATE: $: 22$ -

Note: Food waste, office trash and/or animal carcasses are considered not to contain added radioactivity, and therefore do not require a radiological clearance.

SWO USE ONLY
Lozd Weight (net from scale G estimate) $10 ; 00 \mathrm{C}$

Tru, G 6206884

STaler E 102925

(a) 9:50 


\section{Bechtel Nevadg NTS Landfill Load Verification $1,11^{-05}$}

\begin{tabular}{lllllll}
\hline SWO USE (Circle One Area) AREA & 23 & 6 & 9 & LANDFILL
\end{tabular}

For waste characterizotion, approva! and/or assistance, contact Solid Waste Operation (SWO) at 5-7898. REQUIRED: WASTE GENERATOR INFORMATION

(This form is for rolloffs, dump trucks, and other onsite disposal of materials.)

waste Generator: Buconstruction (mike Kruzic) Phone Number:5-7396) Location / Origin: Area 25 Test cell \& Waste Category: (check one)

Waste Type:

(check one)

THTS Commerclal

(AU -115 nos $7 / 27 / 05^{\prime}$ 7 Tndustrial

$\square$ Putrescible 4 FFACO-onslte

Pollutlon Prevention Category: (check one)

Pollution Provention Catagory: (check one) Method of Characterization: (check one)

$\square$ Asbestoo Containing Materia!

$\square$ FFAGD-offsite WAC Exception Environmental management $\square$ Defenae Projects HIstor) DOE/NV $\exists$ Clean-Up 7 Sampling \& Analysis $\square$ Routine Prohibited Waste at all thrae NTS landfills:

7 Process knowledge VMP

Additional Prohibited Waste Sewage Sludge; Animal carcasses-, Wet garbage (food waste); and Frlable asbeslos at the Area 9 U10C Landfill:

\section{REQUIRED: WASTE CONTENTS ALLOWABLE WASTES}

Check all allowable wastes that are contained within this load.

NOTE: Waste disposed at the Area 8 Hydrocarbon Landfill must have come into contact with petroleum hydrocarbons or coolants such as: gaspline (no benzene, lead); jet fuel: dlesel fuel; lubrlcants and hydraulics; kerosene; asphaltic petroleum hydrocarbon; and ethylene glycol.

Acceptable waste at any NTS landfill:

$\square$ Asphalt $\square$ Metal $\square$ wood $\square$ soil

TPlastio

$\square$ Wire

$\square$ Cable $\square$ Cloth

$\square$ Rocks / unaitered geologie materlals

$\square$ Empty containers

$\square$ Rubber (excluding tires)

$\square$ Insulation (non-Asbestosform)

Demolition debris

$\square$ Manufactured items: (swemp coolers, furniture, rugs, cappet, electronic components, PPE, atc.)

Additional waste accepted at the Area 23 Mencury Landfill: $\square$ Office waste $\square$ Fopd Waste $\square$ Animal Cancases

$\square$ Asbestos: $\square$ Friable $\square$ Non-Friable (contact SwO lif regulated load) Quantity:

Additional waste accepted at the Area $\$$ U10c Landfill:

$\square$ Non-friable asbestos $\square$ Drained automobiles and militery vehicles

$\square$ Light baliasts (contact SWO) $\square$ Drained fuel filters (gas \& cliesel)

$\square$ Hydrucarbons (contact 5Wo) TOther Perlite

Additional waste acceptod at the Area 6 Hydrocarbon Leandfill:

$\square$ septic sludge $\square$ Rags " $\square$ Drained fuọl filters (gas \& diesel)

$\square$ plants $\square$ soil $\square$ sludge from sand/oilwater separators

[.] PCBs below 50 parts per million

Solid fractions from sand/oil/water separators

[C] Deconned Underground and Above Ground

QTanks

$\square$ Other

$\square$ Crushed mon-terne plated oil filters

Initials: (If initialed, no tediological clearanio is necestary.)

The above mentioned waste was generated outsicle of a Controlled Wasto Managi knowledge, does not contalin radiological materiais.

To the best of my knowledge, the waste described above contains only those ma: site. I have varified this through the waste characierlzation method identified abo prohlbited and allowable waste items.
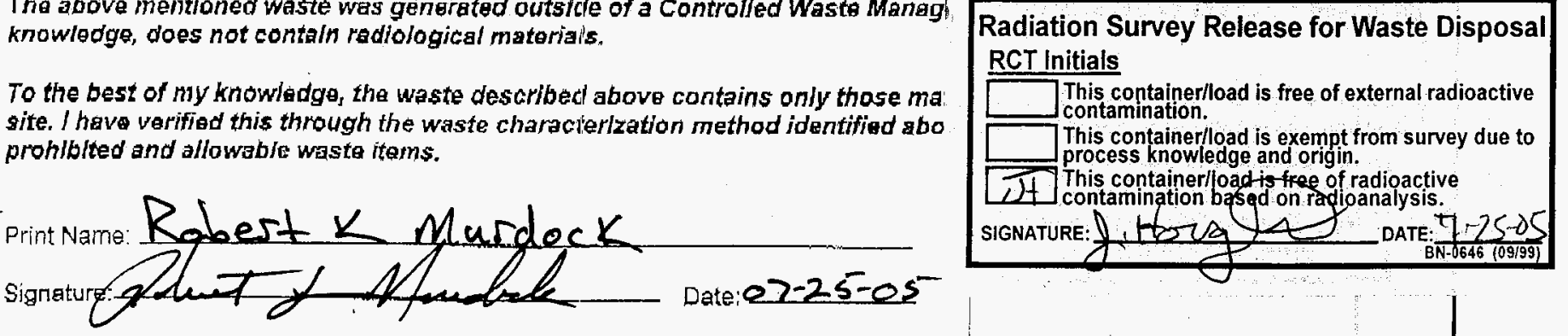

Note: Food waste, office trash and/or animal carossses are considered not to contain added radioactivity, and therefore do not reguirs a radiological clearance.

SWO USE ONLY
Load Weight (net from scale of estimate): 7600

Signature of Certifier:

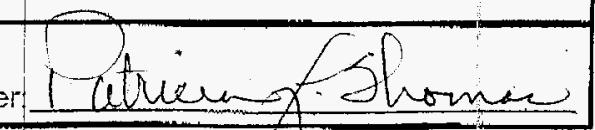

T5uck - G820688A

(2:30

Trailes - E102925

\section{Retantlon Code: ENVE.:}

BN- 


\section{Bechtel Nevada NTS Landfill Load Verification \\ (Waste clefinitions are available on page 2 )}

SWO USE (Circle One Area) AREA $23 \quad 6 \quad 60$ LANDFILL
For waste characterization, approval, and/or assistance, contact Sold Waste Operation (SWO) at 5-7898.

For waste characterization, approval, and/or assistance, contact Solld Waste Operation (SWO) at 5-7898.

REQUIRED: INASTE GENERATOR INFORMATION

(This form is for rolloffs, dump trucks, and other onsite disposal of materials.)

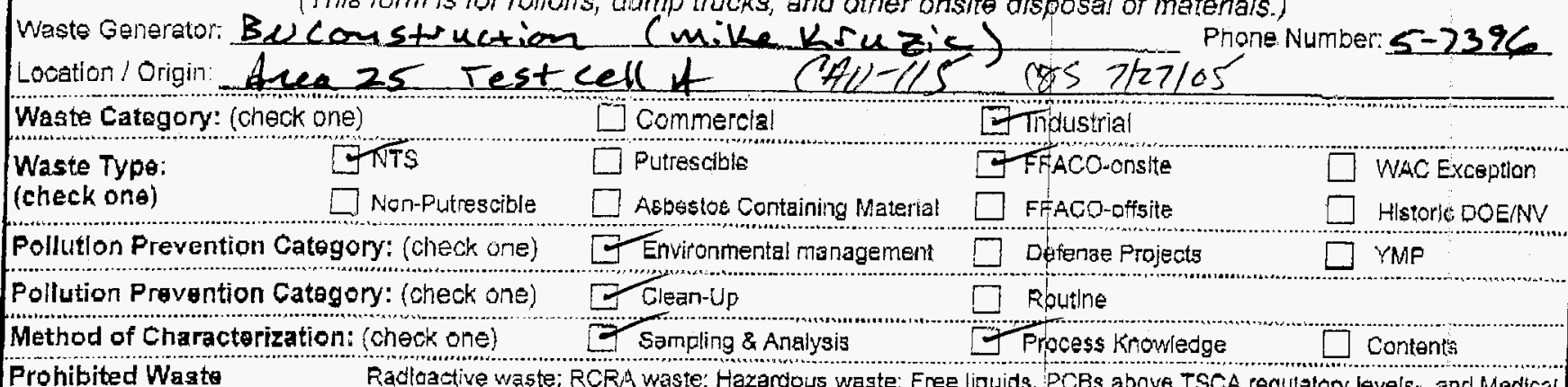
Prohibited Wagte Radloactive waste; RCRA waste; Hazardous waste; Free liquids, PCBs above TSCA regulatory levels., and Medical
at all three NTS landfills:

Additional Prohibited Wazte Sewage Sludge; Animal carcasses-, Wat garbage (food waste); and Frlable asbestoa at the Area 9 Utoc Landffli:

REQUIRED: WASTE CONTENTS ALLOWABLE WASTES

Check all allowable wastes that are contained within this load:

NOTE: Waste disposed at the Area 6 Hydrocarbon Landfill must have come Into contact with petroleum hydrocarbons or coolants such as: gaspline (no benzene, lead); jet fuel: dlesel fuel; lubrloants and hyoraulics; kerosene: asphaltic potroleum irydrocarbon; and ethylene glycol. Acceptable waste at any NTS landfill: $\square$ Paper $\square$ Rocks/ unaitered geologic materlals $\square$ Empty containers $\square$ Asphalt $\square$ Metal $\square$ wood $\square$ Soil $\square$ Rubor (excluding tires) $\quad \square$ Demolition debris Deastic $\square$ wire $\square$ Cable $\square$ ciloth $\square$ insulation (non-Asbestosform)

- Manufactuled items: (swamp coolers, furniture, rugs, carpet, electronic components, PPE, eto.)

Additional waste accepted at the Area 23 Mercury Landfit: $\square$ Office waste W Foọd Waste $\square$ Animal Careasces $\square$ Asbestos: $\square$ Friable $\square$ Non-Friable (contact SWO If regulated load) Quantity:

Additional wasto accepted at the Area 9 U10c Landfill;
Non-friable aspestos
$\square$ Drained automobiles and military vehicles
$\square$ Light ballasts (contact SWO)
D. Drained fuel filters (gas \& diesel)
— Hydrocarbons (contact SWO)
Tother Perlite
—. Solid fractions from sand/oil water siparators
Additional waste acceptad at the Area 6 Hydrocarbon Landfill:
$\begin{array}{ll}\square \text { Septic siudge } \square \text { Rags } & \square \text { Drained fuel filters (gas \& diesel) } \\ \square \text { plants } & \square \text { Soll }\end{array}$
Li Peconned Underground and Above Ground
Tanks
$\square$ Other
$\square$ Crushed non-terne plated oil filters
PCBs below 50 parts per million REQUIRED; WASTE GENERATOR SIGNATURE
Initiais:
(If initialed, no rediological clearanive is necessary.)

The above mentioned waste was generated outsicle of a Controlled Waste Management Area (CWMA) and to the best of my knowledge, does not contaln radiological materials.

To the best of my knowledge, the waste described above contains only those materlals that are allowed for disposal at this

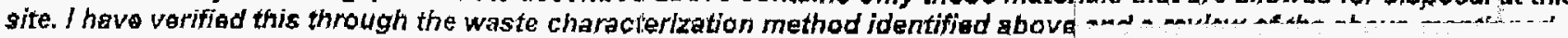
prohiblted and allowable waste itams.

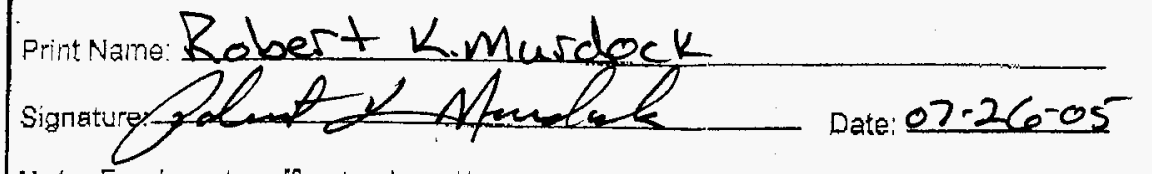

Note: Food waste, office trash andlor animal carcasses are considered not to contein add require a radiological clearance.

SWO USE ONLY
Load Weight (net from soaie ofestimate): $6 / 000$ signature of Certifier

Truck - Elo6400
Radiation Survey Release for Waste Disposal RCT Initials

This container/load is free of external radioactive contamination.

This container/load is exempt from survey due to process knowledge and origin.

This container/load is free of radioactive

Ature contamination based on radioanalysis.

SIGNATURE: 2 a 


\section{Bechtel Nevada NTS Landfill Load Verification (Waste definitions are avaifable on page 2)}

\begin{tabular}{llllll}
\hline SWO USE (Circle One Area) AREA & 23 & 6 & 9 & LANDFILL
\end{tabular}

For waste characterization, approval, and/or assistance, contact Solid Waste Operation (\$WO) at 5-7898. REQUIRED: NASTE GENERATOR INFORMATION

(This form is for rollofis, dump trucks, and other onsite disposal of materials.)

Waste Generator: Bucoustuction (mike Kruzic)
Location / Origin: Area 25 Test cell 4

Prohibited Wagte $\quad$ Radoaetive waste; RCRA waste; Hazandous waste; Free liquids, PCBs above TSCA iegulatory levelsw, and Medical at all throe NTS landfills: wastes (needles sharps, bloody clothing).

Additional Prohibitad Waste Sevage Siudge; Animal carcasses-, Wat garbage (food waste); and Friabls asbeslos at the Area 9 Uioc Landfili:

\section{REQUIRED: WASTE CONTENTS ALLOWABLE WASTES}

Check all allowable wastes that are containod within this load:

NOTE: Waste disposed at the Area 8 Hydrocarbon Landfill must have come into contact with petroleum hydnocarbons or coolants such as: gasbline (no tenzene, lead); jet fuel; dlessl fuel; lubrloants and hydraulics; kerosene: asphaltic petroleum indrocarbon; and ethylene glycol

Acceptable waste at any NTS landfill $\square$ Faper $\square$ Rocks / unatered deologic materlals $\square$ Empty containers
$\square$ Asphalt
$\square$ Metal
L Wood
[.] Soil
$\square$ Rubper (excluding tires)
$\square$ insulation (non-Asbestosform)
Demolition debris
Flastic $\square$ wire $\square$ cable $\square$ ciloth

7 Manufactured items: (swamp coolers. furniture, rugs, carpet electronic components, ppe, etc.)

Addltional waste accepted at the Area 23 Mercury Landfill: $\square$ office waste $\square$ Food Waste $\square$ Animal Carcasses

$\square$ Asbestos: $\square$ Friable $\square$ Non-Friable (contact SWO if regulated load) Quantity:

Additional waste accepted at the Area $\$$ U10c Landfill:

Won-friable asbastos
$\square$ Lignt baliasts (contact SwO) $\square$ Drained autoniobiles and military vehicles
$\square$ Hydrucarbons (contact SWO) $\square$ Other Per liters (gas \& diesel)

Additional waste acceptod at the Area 6 Hydrocarbon Landfill:

$\square$ septic sludge $\square$ Rags $\square$ Drained fugl filters (gas \& diesel)
$\square$ plants $\square$ soll $\square$ siudge from seand/oil/water separators

REQUIRED; WASTE GENERATOR SIGNATURE

Solid fractions from sand/oilwater siparators

[Deconned Underground and Above Ground

$\square$ Tanks

$\square$ Other

I Crushed non-terne plated oil filters

Ińtials:

(If initialed, no rediological cloaranive is neacssary.)

The above mentioned waste wes generated outside of a Controlfed Waste Management Area (CWMA) and to the best of my knowlodge, does not contaln radiological materiais.

To the best of my knowledge, the waste described above contains only those mi site. I have verified this through the waste characterlation method identified abr prohlbited and allowable wasto itoms.

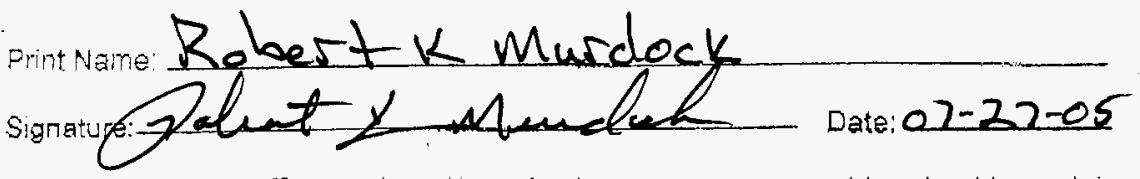

Radiation Survey Release for Waste Disposal RCT Initials

This containerlload is free of external radioactive This container
contamination

This container/load is exempt from survey due to process knowledge and origin.

T This container/load is free of radioactive it This container.oad is ree of radioactive SIGNATURE: tamination based on ran DATE: $17-20$

Note: Food waste, office trash and/or animai corassss are considered not to contain $\varepsilon$ raquire a raciological elearance.

SWO USE ONLY

Trailez - $\$ 2$

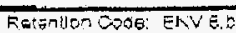

(a) 3:00

Truak. E Elo6400 
Bechtel Nevade

SWO USE (Circle One Area) AREEA
NTS Landfill Load Verification

(Waste definitions are available on page 2)

For waste characterization, approva!, and/or assistance, contact Soljd Waste Operation (SWO) at 5-7898.

REQUIRED: NASTE GENERATOR INFORMATION

(This form is for wlloffs, dimp trucks, and other onsite disoosal of materials.)

Waste Generator: BUconstruction (mike kruzic)

Waste Category: (check one) [ Commercial

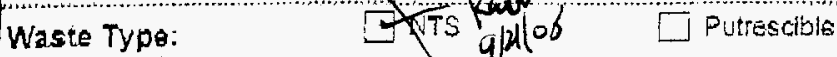

(check one)

Nen-Futrescible

Pollution Prevention Category: (check one)

Pollution Provention Catagory: (check one)

Method of Characterization: (check one)

Astostos Containing Materia!

Environmental management

Clean-Up

7 Sompling \& Analysis

6

$(4,-6)$

$r$

9

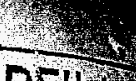

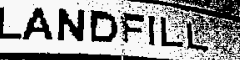

Phone Number: $5-2396$

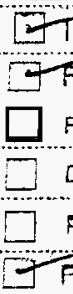

Thidustrial

TFACo-onsite

WAC Exception

FFACD-Dffsite

HIstorlc DOE/NV

Dofenre Projects

YMP

Routlme

Process Knowledge $\square$ Contents

Prohibited Waste

Radloactive waste; RCRA waste; Hazardous waste; Free liguids, PCBs above TSCA pegulatory leveis", and Medice at all throe NTS landfilis: wastes (needles; sharps, bloody clothing).

Additional Prohibited Waste Sewage Sludge; Aninal carcasses-, Wat garbage (food waste); and Frlable asbeslos at the Arsa 9 U10c Landfill:

\section{REQUIRED: WASTE CONTENTS ALLOWABLE WASTES}

Check all allowable wastes that are contains within this load.

NOTE: Waste disposed at the Area 8 Hydrocarbon Landill nust have come into contact with petroleum hydrocarbons or coolants such as: gafeline (no benzene, lead); jet fuel: dlesel fuel; luorlcants and hydraulics; kerosene: asphaltic potroloum hydrocarbon; and ethylene glycol

Acceptable waste at any NTS landfill: $\square$ Faper $\square$ Rocks / unaltered geologie materlals $\square$ Empty containers

$\square$ Asphalt $\quad \square$ Metal $\square$ wood $\square$ soil $\square$ Rubber (excluding tires)

ZFlastic $\square$ Wire $\square$ Cable $\square$ Cloth $\square$ insulation (mon-Asbestosform)

Demolition debris

Cement \& soncrete

D Manufactured itams: (swamp coolers, furniture, rugs, carpat, electronic components, PPE. etc.)

Additional waste accepted at the Area 23 Mereury Landfill: $\square$ Offico waste $\square$ Food Waste $\square$ Animal Carcasces

$\square$ Asbestos; $\square$ Friable $\square$ Non-Friable (contact SWO If regulated load) Quantity.

Additional waste acsepted at the Area 9 U10c Landfill:

[Non-friable asbastos $\square$ Drained automobiles and militery vehicles

Ш Light ballasts (contact SWO) $\square$ Dreined fuel filters (gas \& diesel)

$\square$ Hydrocarbons (contact SwO) ZOther Perlite

Additional waste acceptod at the Area 6 Hydrocarbon Landfill:

$\square$ septic sludige $\square$ Rags

- Diained fuel filters (gas \& diesel)

$\square$ Sludge from send/oil/water separators

REQUIRED: WASTE GENERATOR SIGNATURE

Initials fif initialed, no rediological elearanice is necestary.

The above mentioned waste was generated outside of a Controlled Waste Mananamant a noa /Clumal and to the hael nf mu knowledge, does not contaln radiological materiais.

O the best of my knowledge, the waste described above contains only thase $n$ site. I have verified this through the waste characlerization method identifiad a. prohlolted and allowab/e waste itams.

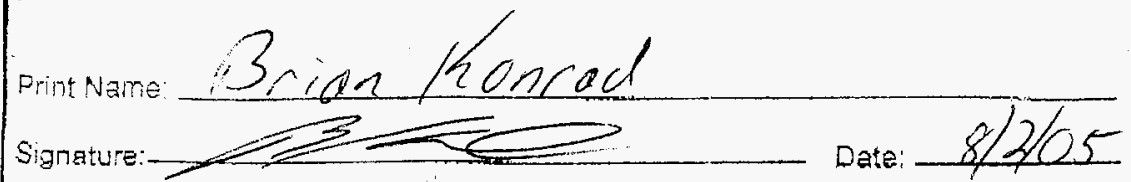

Radiation Survey Release for Waste Disposal RCT Initials

This container/load is free of external radioactive contamination.

This containerload is exempt from survey due to process knowledge and origin.

II This containerlload is free of radioactive

If contamination based trimadioanatysis.

SIGNATURE:

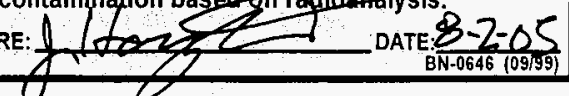

Note: Food waste office trash andior animal caroassas are consideied not to contain added radioactivity, and therafcre do net raguire a radiological clearsnce.

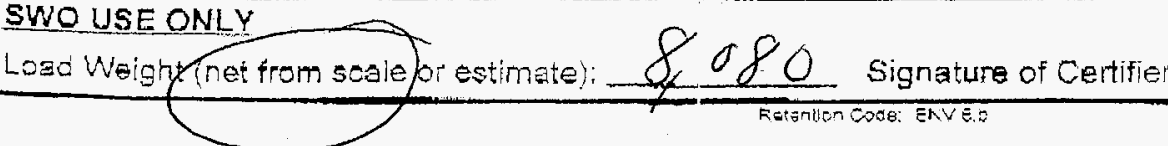

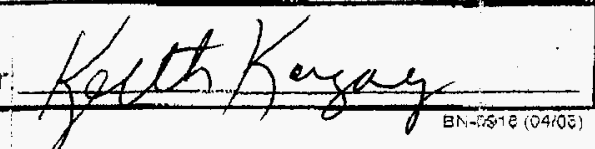


Bechtel Nevada

SWO USE (Circle One Area) AREEA
NTS Landfill Load Verification

(Waste definitions are avaliable on page 2)

For waste characterization, approva! andror assisfance, contact Solid Waste Operation (\$WO) at 5-7898.

REQUIRED: NASTE GENERATOR INFORMATION

(This form is for milofis, dump trucks, and other onsite disposal of materials.)

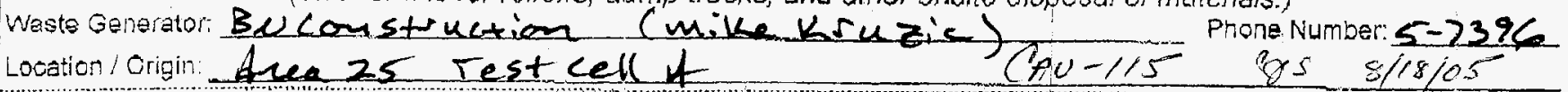

Waste Category: (check one)

Waste Type: WNS

(check one)

Non-Futrescible

[.] Commerela!

$\square$ Putrescibie

tindustrial

FFACO-nsite

WAC Excoption

Pollution Prevention Catagory: (check une)

Pollution Provontion Catogory: (check one)

Method of Characterization: (check one)

I Aspaslos Containing Material

$\square$ FFACO-Ditsite

$\checkmark$ Environmental management

- Glean-Up

7 Sampling \& Analysis

Defenge Projects

HIstoris: DOENV YMF

\section{Prohibited Wasto}

at all thrae NTS landfils:

Contents

Additional Prohibited Waste Sewage Siudge; Animal carcasses-, Wat garbage (food waste): and Frlable asbeslos at the Area 9 U10e Landfili:

\section{REQUIRED: WASTE CONTENTS ALLOWABLE WASTES}

Check all allowable wastes that aro containod within this toad

NOTE: Waste disposed at the Area 9 Hydrocarbon Landfil nust have come Into contact with petroleum hydnacarbons or coolants such as: gaspline (no benzene, lead) jet fuel desel fuel; lubricants and hydraulics kerosene: asphaltic potroleum hydrocarbon; and ethylene glycol

Acceptable waste at any NTS landfill $\square$ Faper $\square$ Rocks / unaltered geologie materlals $\square$ Empty containers

E Asphalt $\square$ Metal $\square$ wood $\square$ soil $\square$ Rubber (excluding tires)

Plastic $\square$ wire $\square$ cable $\square$ ciloth $\square$ Insuletion (non-Asbestosform)

$\square$ Demolition debris

7 Manufactured items: (swemp coolers, fumiture, rugs, carpet, electronic components, PPE, etc.)

Additional waste accepted at the Area 23 Mercury Landfil: $\square$ Offico Waste $\square$ Food Waste $\square$ Animal Carcasses

[ Asbestos: $\square$ Friable $\square$ Non-Friable (contact SWO lf regulated load) Quantity:

Additional waste aceepted at the Area 9 U10c Landfill:

\begin{tabular}{|c|c|c|}
\hline [Non-friable asbabtos & $\square$ Drained automobiles and military vehicles & $\square$ Solid fractions from sand/oil/water seperators \\
\hline$[$ Lignt ballasts (contact SWO) & Drained fuel filters (gas \& diesel) & [ Poconned Underground and Above Ground \\
\hline Hydrocarbons (contact SWO) & Other & $\square$ Tanks \\
\hline Additional waste accoptod at the & a Area 6 Hydrocarbon Landfill: & $\square$ Other \\
\hline$\square$ septic sludge $\square$ Rags & $\square$ Diained fuel filters (gas a diesel) & Crushed non-teme plated oil filters \\
\hline Dplants $\quad \square$ soil & [ Siudge from sandroiliwater separators & PCBs below 50 parts per million \\
\hline
\end{tabular}

The above mentioned waste was generated outsicle of a Controlled Waste Mane knowleoge, does not contaln rediological materials.

To the best of my knowledge, the waste described above contains only those $m$ site. I have verified this through the waste characrerlzation method identified ab prohlolted and allowabie wasto itams.

Print vame: Robert $k$ Murdock

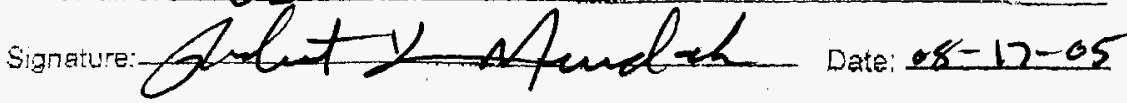

Radiation Survey Release for Waste Disposal RCT Initials

This container/load is free of external radioactive contamination.

This container/load is exempt from survey due to process knowledge and origin.

TH This containerlload is free of radioactive

LI T contamination haseton radioanalysis.

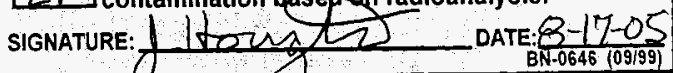

Note: Food waste, office trash andror animal carcassiss are considered not to contein added radioactivity, and tharefore do nct requins a radiological clearance.

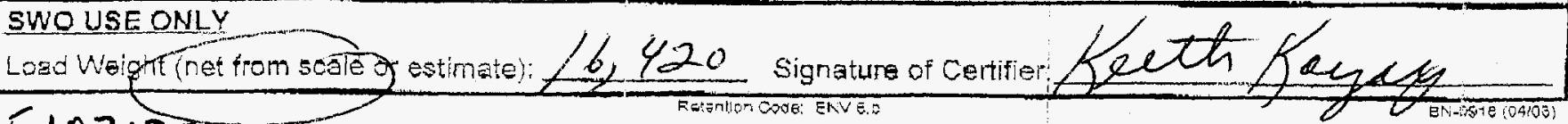

E107170-TSuck

33 - Trailen 
NTS Landfill Load Verification (Waste tefinitions are available on page 3)

SWO USE (Circle One Area) AREA 23

6

9

LANDFILL

For waste characterization, approva!, and/or assistance, contact Solid Waste Operation (SWO) at 5-7898. REQUIRED: NASTE GENERATOR INFORMATION

(This form is for rollofis, dump trucks, and other onsite disposal of materials.)

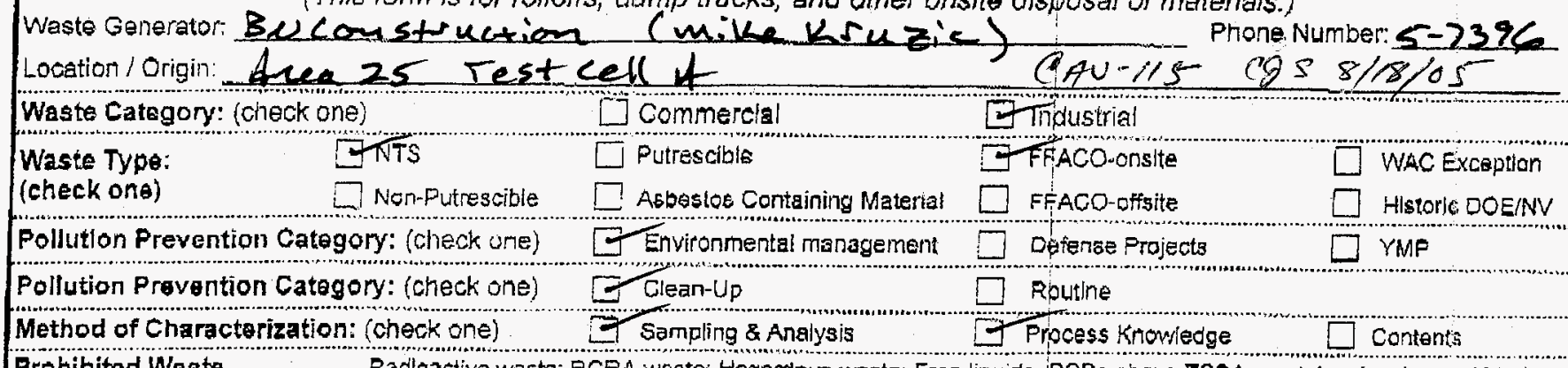
Frohibited Wagte
at all three NTS landfills;

Adtitional Prohibited Waste Sewage Sludge; Animal carcasses-, Wat garbage (food waste); and Frlable asbestos at the Area 9 U10c Landfill:

REQUIRED: WASTE CONTENTS ALLOWABLE WASTES

Chrock all allowable wastes that ar contained within this load:

NOTE: Waste disposed at the Area 8 Hyerocarbon Landfill must have come into contact with petroleum hydnocarbons or coolants such as: gaspline (no benzene lead); jet fuel: desel fuel; lubricants and hydraulics; kerosene: asphaltic pefjolaum hydrocarbon; and ethylene glycol.

Acceptable waste at any NTS landfill: $\square$ Paper $\square$ Rocks / unaitered geologie materlals

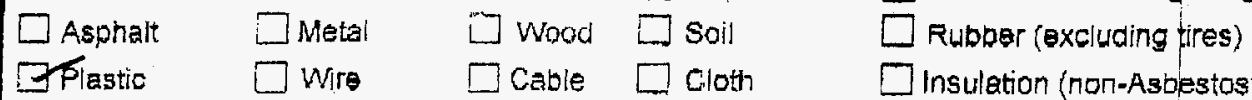

$\square$ insulation (non-Asbestosform)

Empty containers

$\square$ Demolition debris

7 Manufactured items: (swemp coolers, furniture, rugs, carpet, electronic components, PpE, etc.)

Additional waste accepted at the Area 23 Mercury Landfill: $\square$ Office waste $\square$ Food Waste $\square$ Animal Careasces

$\square$ Asbestos: $\square$ Friable $\square$ Non.Friable (contact Swo if regulated load) Quantity;

Additional was to accepted at the Area 9 U10c Landfill:

Non-friable asbastos $\square$ Drained autoniobiles and military vehicles

$\square$ Light ballasts (contact SWO) [ Drained fuel filters (gas \& diesel)

Hydrucarbons (contact swo) TOther Perlite

Additional waste acceptod at the Area 6 Hydrocarbon Landfill:

$\square$ Septic sludge $\square$ Rags $\square$ Drainad fuel fiters (gas \& diesel)

$\square$ Plants $\quad \square$ soil $\square$ sludge from sand/oil/water separators

REQUIRED: WASTE GENERATOR SIGNATURE

initials:

(If initialed, no rediologtical cloaranive Is necestary.)

Tha above mentioned waste was generated outsicie of a Controlled Waste Manage knowledge, does not contaln radiological materiais.

To the best of my knowledge, the waste described above contains only those mat site. I have varifiad this through the waste characterlzation method jdentified abov prohlbited and allowable waste items.

Print Name: Robert K. Murdock signature: Tobut y Mardack Date: 08-17-05

Radiation Survey Release for Waste Disposal RCT Initials

This containerlload is free of external radioactive contamination.

This container/load is exempt from survey due to process knowledge and origin. it This containerlload is free of radioactive

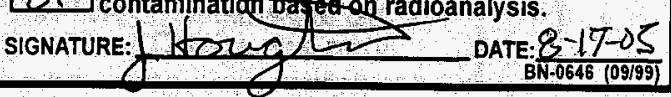

Note: Food waste, office trash andlor animal carcasses are considered not to contain added radioactivity, and therpfore to net raguire a radiological elearance.

LWO USE ONLY

Truck - E107170

Rerenllon Code: ENve.

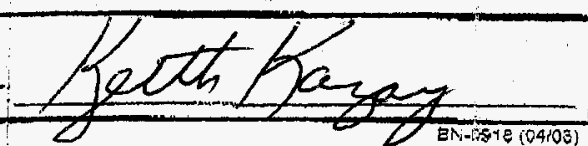

Trailes - 08 
NTS Landfill Load Verification

(Waste tefinitions are avaliable on page 2)
Bechtel Nevade

SWO USE (Circle One Area) AFEA
23
6

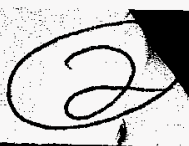

For waste characterizetion, approva!, andfor assistance, contact Solio Waste Operation (SWO) at 5-7898.

REQUIRED: NASTE GENERATOR INFORMATION

(This form is for rollofis, dimp trucks, and other onsite disposal of materials.)

Waste Generator: BUConstuation (mike Lruzici)

Lowation/Origin: Area 25 Test cell 4

Waste category: (check one)

Waste Type:

\section{NTS}

D. Nen-Futrescible

(check one)

Pollution Prevention Category: (check une)

Pollution Prevontion Catagory: (check one)

Method of Characterization: (check one)

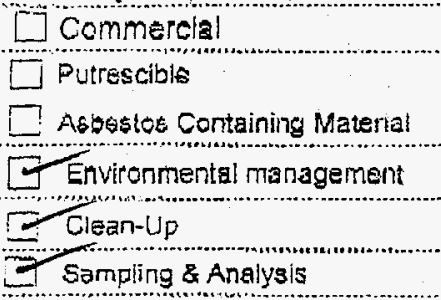

\section{os}

Thdustria

Prohibited Waste
at all thrae NTS landfills:

Phone Number: $5-23 \%$

LANDFILL

9

\begin{tabular}{ll}
\hline FFACO-ansite & $\square$ WACException \\
$\square$ FFACO-Difsite & $\square$ HIstoric DOENV \\
$\square$ Defenge Projeds & $\square$ YMP \\
$\square$ Routhe & $\square$ Contents
\end{tabular}

Additional Prohibitod Wagte Sevage Sludge; Animal carcasaes-, Wat garbage (food waste); and Frlable asbeslos

at the Area 9 Utoc Landfill:

REQUIRED: WASTE CONTENTS ALLOWABLE WASTES

Chock all allowable wastes that are contains d within this load:

NOTE: Waste disposed at the Area E Hydrocarbon Landfill nust have come into contact with petroleum hydrocarbons or coolants such as:

gaspline (no benzene, lead); jet fuel: desel fuel; lubrlcents and hydraulics; kerosene: asphalfic petrolaum hydrocarbon; and ethyleng glycol.

Acceptable waste at any NTS landfill: $\square$ Faper $\square$ Roeks / unaltered geologic materlals $\square$ Empty coritainers

$\square$ Asphalt $\square$ Metal $\square$ wood $\square$ soil $\square$ Rubber (excluding tires) D Demolition debris

Bplestio $\square$ Wiro $\square$ cable $\square$ cioth $\square$ insulation (non-Asbestosform)

Cement 8 concrete

Wianufactured items: (swamp coolars. furniture, rugs, carpat, electronic components, PPE, atc.)

Additional waste accepted at the Area 23 Mercury Landfill: $\square$ Offica waste $\square]$ Food Waste $\square$ Animal Careasses

$\square$ Asbastos: $\square$ Friable $\square$ Non-Friable (contact SWO If regulated load) Quantity

Additional waste acepted at the Area 9 U10c Landfill:

Non-friable asbastos $\square$ Drained automobiles and militery vehicles

W Light ballasts (contact swo) T Draned fuel filters (gas \& diesel)

ZHydrucarbons (contact swo) TOther Perlite

Additional waste acceptad at the Area 6 Hydrocarbon Landfill:

$\square$ septic sludgs $\square$ Rags $\square$ Drained fual filters (gas \& diesel)

$\square$ Plants $\square$ soll $\quad$ Siludge from sandioilwater separators

REQUIRED; WASTE GENERATOR SIGNATURE

Initials:

(If initialed, no rediologicgl doaranite is necestary.)

The above mentioned waste was generated outside of a Controlied Waste Management Area (CWMA) and to the best of my

knowledge, does not contaln radiologital materiais.

To the best of my knowiedge, the waste described above contains only those mat site. I heve verified this through the waste characierization method identified abo: prohlibled and allowabie wasto itams.
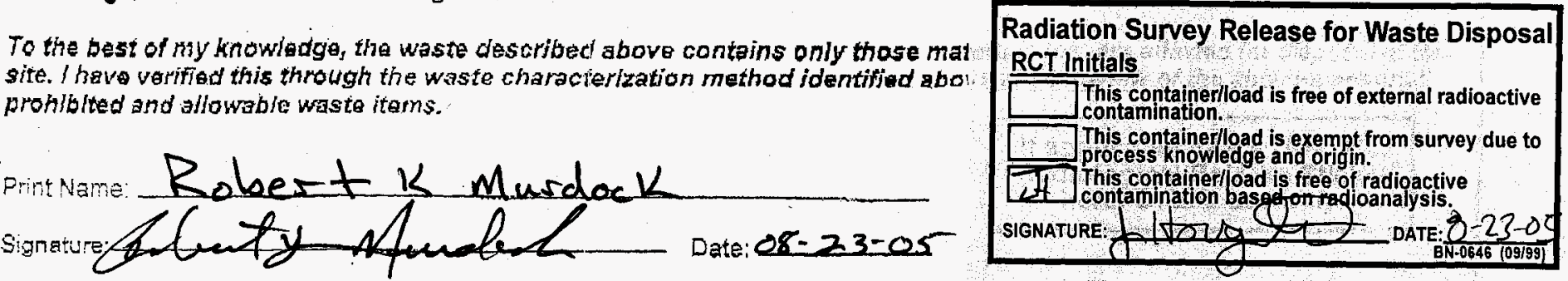

Note: Food waste, office tresh andior animal carosssas are considered not to contain added radioactivity, and thereiore wo he? rsquire a raciologica clearance.

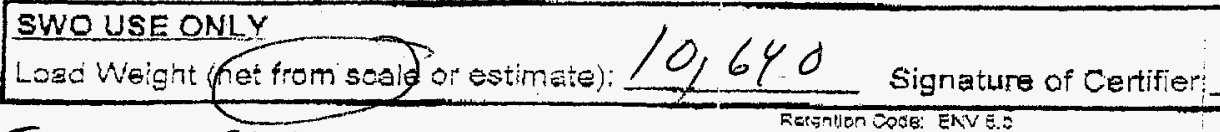

Tsuck E107170

Solid fractions from sand/oilwater separators

L Deconned Underground and Above Ground

$\square$ Tanks

Other

- Crushed non-tarne plated oil filters

PCBs bejow 50 parts per million 
For wasto characterization, approval, andror assistance, contact Solid Waste Operation (SWO) at 5-7893.

REQUIRED: NASTE GENERATOR INFORMATION

(This form is for rallofis, clump trucks, and other ansite disposal of materials.)

Wast Generator Buconstruction (mike bruzic) phone Number: $5-2396$ Lotation/Origin Area 25 Test cell 4 Cav-Cl Waste Category: (check one)

Waste Type:

(check one)

$\longrightarrow$ NTS

- Non-Putrescitle

Pollution Prevention Catagory: (check une)

Pollution Provantion Catogory: (check one) Method of Charactorization: (chack one)

Prohibited Waste

at all thrae NTS andfilis:

Radloactive waste. FCP A............

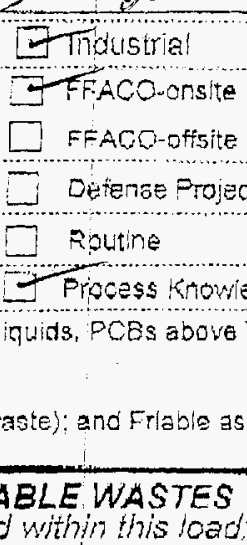

Additional Frohibitad Waste sewage Siudge; Alinal carcasses., Wet garbage (food waste); and Frlable astestos at the Area 9 U10c Landfill:

\section{REQUIRED: WASTE CONTENTS ALLOWABLE WASTES
CiJeck all allowable wastes that are containsd within this load:}

NOTE: Waste disposed at the Area 6 Hydroserbon Lendfill must have come into contat with petroleum hydrocarbons or coolants such as:

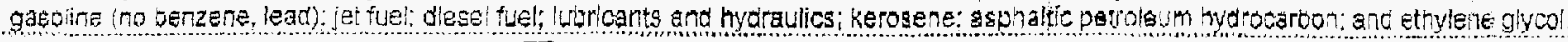
Acceptable waste at any NTS landfill: $\square$ Faper $\square$ Rocks/Hnaitered geologie materials

\section{$\square$ Asphalt $\quad \square$ Metai $\square$ wood $\square$ soil $\square$ Ruboer (excluding tires)}

JPlastio

L Wire

$\square$ caile $\square$ Cloth

Dinsulation (non-Asbestosform)

$\square$ Empty containers

$\square$ Demolition debris

$\square$ Cement concrate

- Manuactured tems: (swamp coolers. furniture rugs, carpat, electronic components, PPE etc.)

Additional waste accepted at the Area 23 Mereury Lanelfill: $\square$ Office waste D Food Waste I Animal Careasses

[ Asbestos: EFriable E Non-Friable (contact Swo if regulated load) Quantity:

Additional wast aceepted at the Area $\$$ U10c Landfill:

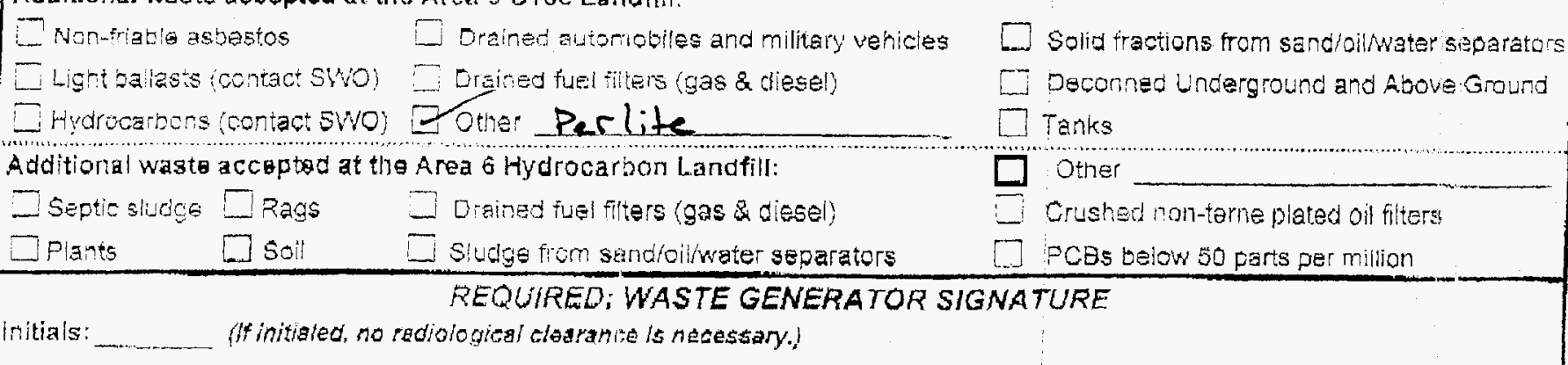

The above mentioned waste was generated outsicie of a Controlled Waste Manag knowledge, does not contain radiological materiais.

To the best of my knowladge, the waste described above contains only those ma site. I have varified this through the waste characierlzation method identified abc prohilited and allowable wasto itams.
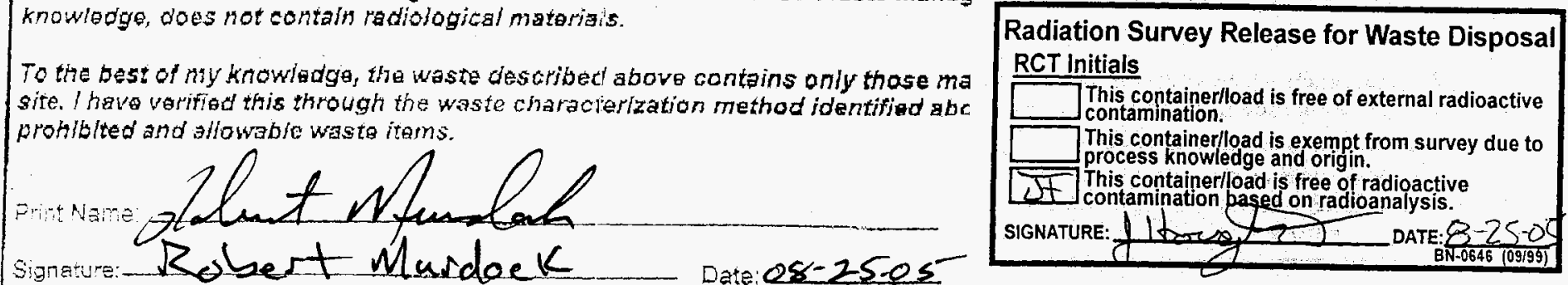

Note: Food waste, office trash andior animal carasses are consideied not to contrin added radioactivity, and tharefore do met require a radiological clearince

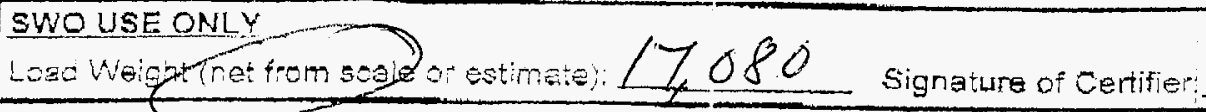

Truck - E107170

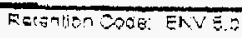

Truilen- 
$04 / 25 / 2065 \quad 15: 26 \quad 17020957562$

Bechtel Nevadg NTS Landfill Load Verification

Waste clefinitions are avaliable on page 2)

SWO USE (Circle One Area) AREA $\quad 23 \quad 6$

9

LANDFILL

For waste characterization, Gpprova! andror assistance, contact Solid Waste Operation (\$WO) at 5-7896.

REQUIRED: NASTE GENERATOR INFORMATION

(This form is for rollofis, clump trucks, and other onsite disposal of materials.)

waste Generator Buconstruction (mike ksuzic)

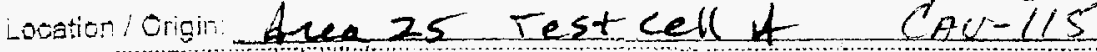
Waste Category: (chack one) / kad [D Commercial Waste Type: $\quad \exists$ TIS $10 / 105$ Q (check one) L Nen-Futrescible

Pollutlon Prevention Gategory: (check ume)

Pollution Pravention Catagory: (check one)

Method of Charactorization: (check one)

I Aspestoe Containing Material

2 Environmental management

Ciean-Uo

2 C

Sampling \& Analysis

Phone Number: $5=2396$

Prohibited Waste

Padloative waste; RCRA weaste; Hazandous waste; Fre liquids, PCBS above TSCA requiatory leveis, and Medical

at all throe NTS landfills: wates (needies sharps, bloody clothing)

Additional Prohibited Waste Sevage Siudge; Animal carcassas-, Wat garbage (food waste): and Frlable asbeslos at the Area 9 U10C Landfill:

\section{REQUIRED: WASTE CONTENTS ALLOWABLE WASTES}

Chach all allowable wastes that ars containsd within this load

NOTE: Waste disposed at the Area 5 Hycrocarbon Landfil must have come mo contast with petroleum hydrocarbons or coolants such as:

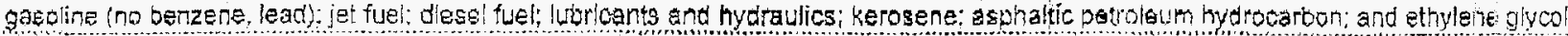

Acceptable waste at any NTS Iandfill: $\square$ Figer $\square$ Rocks/ wnaitered geologie materlals $\square$ Ëmpty containers

[ Aspralt $\square$ Metal $\square$ wood $\square$ soil $\square$ Ruboer (excluding tires)

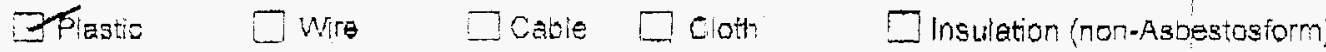

Demolition debris

I Manutactured items: (swemp coolers, furniture, rugs, carpet, electronic components, PPE, atc.)

Additional waste accepted at the Araa 23 Mereury Landill: $\square$ ofice waste $\square$ Food Waste $\square$ Animal Careasses

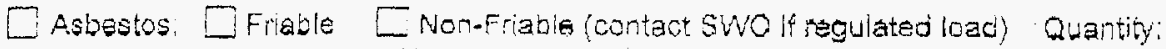

Additional waste aceepted at the Area 9 U10c Landifli:
INenfriable asbestos
- Dreined automobiles and military venicles
Zugnt bailasts icontact Swo
Eraned fuel filters (gas \& diesel)
[Hydrocarbons (contact Ewo)
- Other Perlite
Additionel waste acceptod at the Area 6 Hydrocarbon Landfill:

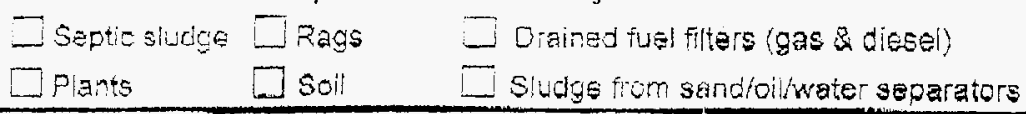
D. Solid tractions from sand/oilhater siparator
F Daconned Underground and Above Ground
$\square$ Tanks
E Other
Crushed non-terne plated oil filters

REQUIRED; WASTE GENERATOR SIGNATURE

Intials (ff initialed, no rediological cloaranise is Metestary.)

The above mentfoned waste was generated outside of a Controlled Waste Managemant Area (CWMA) and to the best of my knowledge, oles not contain radiologital materiais.

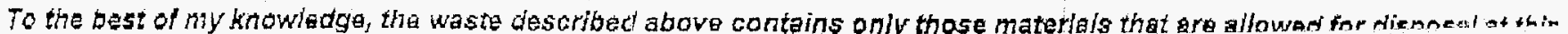
site. I heve verified this through the waste characterlation method identified a pronlbled and allowable wasta itams.

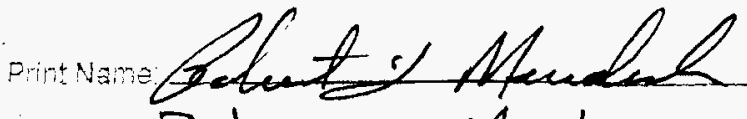

Signature: Robert K Musdock Date: $q-0 /-05$

Note: Food waste, office trash andiry animal carabsos are considered not to contair recuire a rodiological clearance.

\section{SWO USE ONLY}

Load Weight inet from soale or estimatei 6,60 signature of Certifier:
TIK: $E 106400$
$8: 20 \mathrm{fin}$

Radiation Survey Release for Waste Disposal

RCT Initials This container

This container/load is exempt from survey due to This container/load is exempt
process knowledge and origin

This container/load is free of radioactive

SIGNATURE: Tontamination based on radioanalysis. $9 / 1 / 05$

TH: 29 


SWO USE (Circle One Area) AREA $23 \quad 6 \quad 20$ LANEFILL

For waste characterization, approva!, andjor assistance, contact Solid Waste Operation (SWO) at 5 -7898.

REQUIRED: NASTE GENERATOR INFORMATION

(This form is for rollofis, dump trucks, and other onsite disposal of materials.)

waste Generator BuConstuction (mike bruzic) Phone Number: $5-23 \%$

Location / Origin Acea 25 Test cell \& $C A i-1 / 5$

Waste Category: (check one)

Waste Type:

(check one)

3 KTS Kawjotos
$\square$ Non-Putrescible

[] Commerela!

$\square$ Putrescibis

Pollution Prevention Category: (check une)

Pollution Provention Category: (check one)

Method of Characterization: (check one)
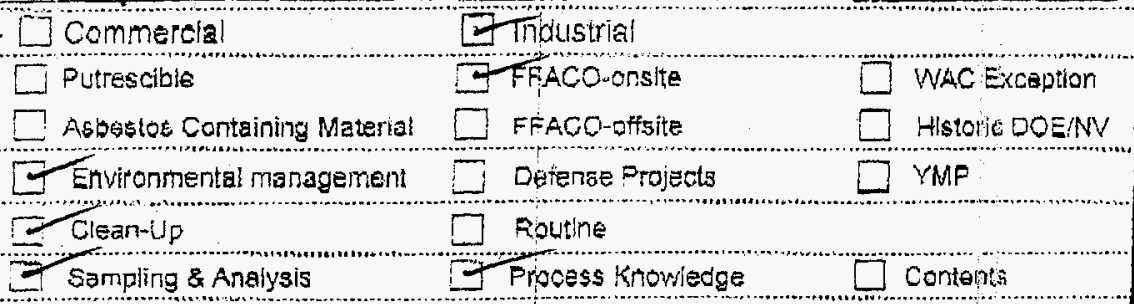

Prohibited Waste

Radloative waste; RCRA waste; Hazanous waste; Free liquids, PCBs above TSCA pegulatory levels-and Medical at all three NTS landfills:

wastes (needles smarps, bloody clothing).

Additional Prohibito Whaste Sevage Sludge; Animal carcasses-, Wet garbage (food waste); and Frlable asbestos

at the Area o U106 Landfill:

REQUIREO: WASTE CONTENTS ALLOWABLE WASTES

Check all allowable wastes that are contains d within this load:

NOTE: Waste disposed at the Area 0 Hydrocarbon Lendfill must have come into contact with petroleum hydrocarbons or coolants such es:

gaspline (no benzene, lead): jel fuel: dlesel fuel; ludploants and hydraulics; kerosene: asphalic potroleum hydrocarbon; and ethyleite glycol

Acceptable waste at any NTS Iandfill: $\square$ Faper $\square$ Rocks / unaitered geologie materlals $\square$ Enpty obntainers
$\square$ Asphait
$\square$ Metal
$\square$ word
$\square$ soil
$\square$ Rubper (excluding tires)
- Demolitian debris

Flestio

$\square$ Wiro

$\square$ Cable

Lioth

Onsulation (non-Asbestosform)

I Manuactived items: (swamp coolers. fumiture, rugs carpet, electronic components, ppe, etc.)

Addlional waste accepted at the Araa 23 Mercury Landfill: $\square$ Offico waste $\square$ Food Waste $\square$ Animal Careasses

$\square$ Asbestos: $\square$ Friable $\square$ Non-Friable \{contact 5 WO if negulated load $\}$ Quantity:

Additional was acoepted at the Area $\$$ U10c Landfill:

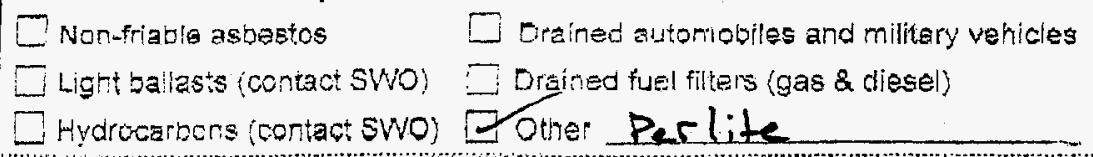

Additional waste acceptod at the Area 6 Hydrocarbon Landfill:

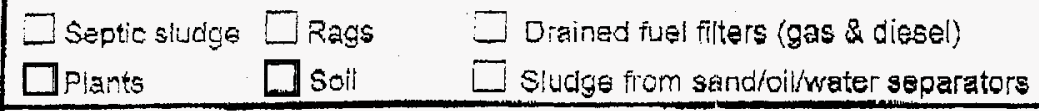
REQUIRED: WASTE GENERATOR SIGNATURE

Initials: (If initialed, no rediological cloaranie is necestary.)

Tha above mentioned waste was generated outside of a Controlled Waste Management Area (CWWA) and to the best of my knowledge, does not contaln radiological materiais.

To the best of my knowiadge, the waste described above contains only those materlels that are allowed for disposal at this site. I have varified this through the waste characierization method identifiad above and a revlew of the above-mantioned prohlbited and allowable wasto itams.

Print Name: require a radiological clearance.
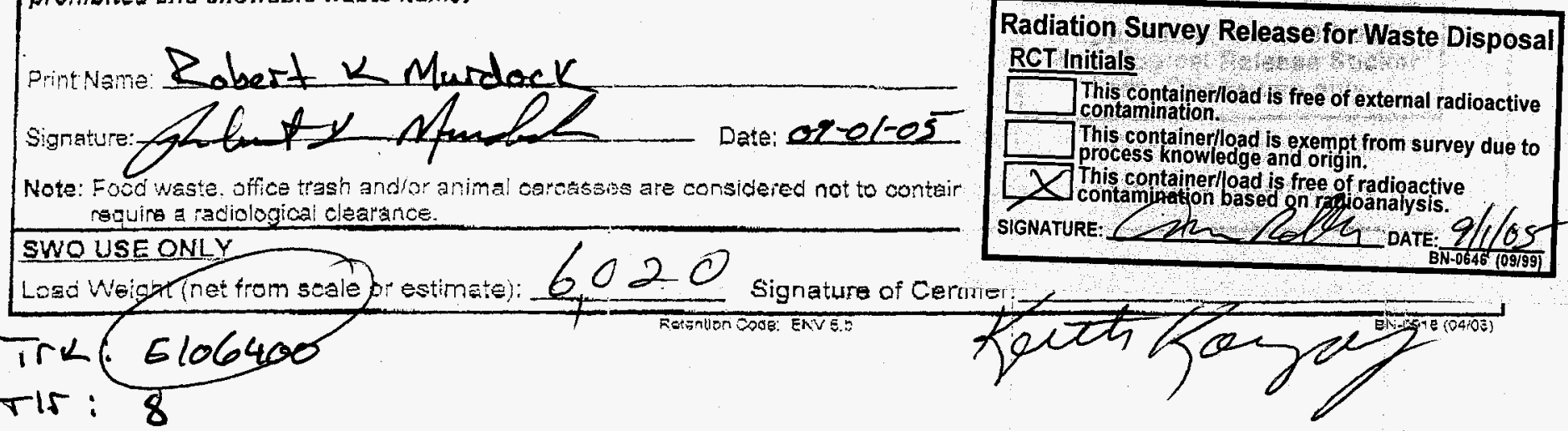

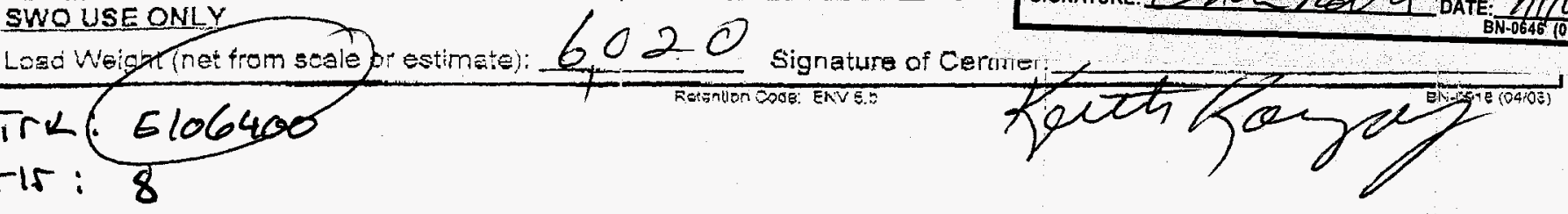


Please print or type. (Form designed for use on elite (12-pitch) typewriter.)

\section{UNIFORM HAZARDOUS WASTE MANIFEST}

3. Generator's Name and Mailing Address

4. Generator's Phone ( $702,295-0311$

5. Transporter 1 Company Name MP Environmental Services

7. Transporter 2 Company Name 9. Q Us ignated Facility Name and Site Address

Hwy 95, 12 miles South of Beatty

Beatty, NV 89003
1. Generator's US EPA ID No.

NV3890090001

Form Approved. OMB No. 2050-0039.

Bechtel Nevada for US DOE
P.O. BOX 98521 , m/s NTS 110
Las Vegas, NV 89193

Manifest

Document No.

05020

2. Page 1 Information in the shaded areas of 1 is not required by Federal law.

A. State Manifest Document Number

Las Vegas, NV 89193

B. State Generator's ID

C. State Transporter's ID

D. Transporter's Phone (305) 458-3036

E. State Transporter's ID

F. Transporter's Phone T

G. State Facility's ID

10. US EPA ID Number

H. Facility's Phone

( 800$) 239$ - 3943

11. US DOT Description (Including Proper Shipping Name, Hazard Class and ID Number)

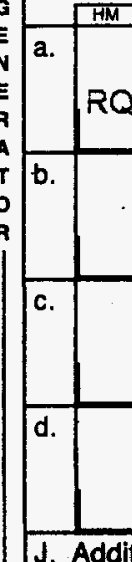

NVT 33000100000

\begin{tabular}{|c|c|c|c|c|}
\hline \multicolumn{2}{|c|}{$\begin{array}{c}\text { 12. Containers } \\
\text { No. }\end{array}$} & $\begin{array}{c}\text { Type } \\
\text { Total } \\
\text { Quantity }\end{array}$ & $\begin{array}{c}\text { Un } \\
\text { Whit }\end{array}$ & Waste No. \\
\hline 1 & CM & 3000 & $P$ & 0006 \\
\hline & & & & \\
\hline & & & & \\
\hline & & & & \\
\hline
\end{tabular}

J. Additional Descriptions for Materials Listed Above

K. Handling Codes for Wastes Listed Above

A: ERG 171;EN-NTS-05-0087; 13-3388

B:

C:

D:

15. Special Handling Instructions and Additional Information

Shipment origin: BX for TSDOE. Wevada Test Site, Hay 95 . Mercury, NV 89023.

24-hour emergency contact \# (702) 295-0311 (call collect).

Use Proper PPE when handing containers.

Certificate or Destruction is requitred.

16. GENERATOR'S CERTIFICATION: I hereby declare that the contents of this consignment are fully and accurately described above by proper shipping name and are classified, packed, marked, and labeled, and are in all respects in proper condition for transport by highway according to applicable international and national government regulations.

If I am a large quantity generator. I certify that I have a program in place to reduce the volume and toxicity of waste generated to the degree I have determined to be economically practicable and that 1 have selected the practicable method of treatment, storage, or disposal currently available to me which minimizes the present and future threat to human health and the environment; OR, if I am a small quantity generator, I have made a good faith effort to minimize my waste generation and select the best waste management method that is available to me and that $I$ can afford.

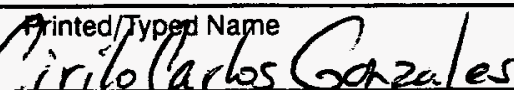

T 17. Transporter 1 Acknowledgement of Receipt of Materials

Printed/Typed Narno

: 2014

18. Transporter 2 Acknowledgement of Receipt of Materials

Printed/Typed Name

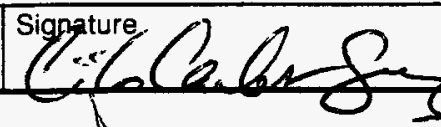

Month. Day/ Year $101499+1015$

E

\section{Discrepancy Indication Space}

f. 13a) Weight Received $2220 \mathrm{P} M$

20. Facility Owner or Operator: Certification of receipt of hazardous materials covered by this manifest except as noted in Item 19.
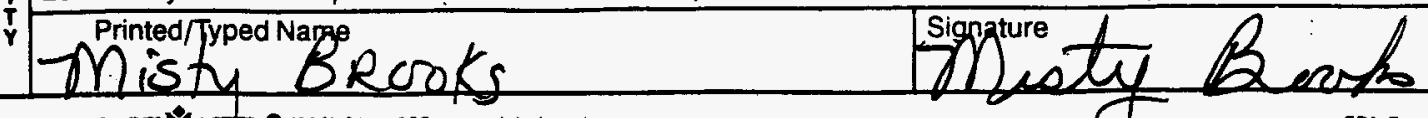

Month Day Year

Style F15 LABELY LASTER (800) 621-5808 umw.labelmaster.com

EPA Form 8700-22 (Rev 9-88) Previous editions are obsolete. 
Please print or type. (Form designed for use on elite. tch) typewriter.)

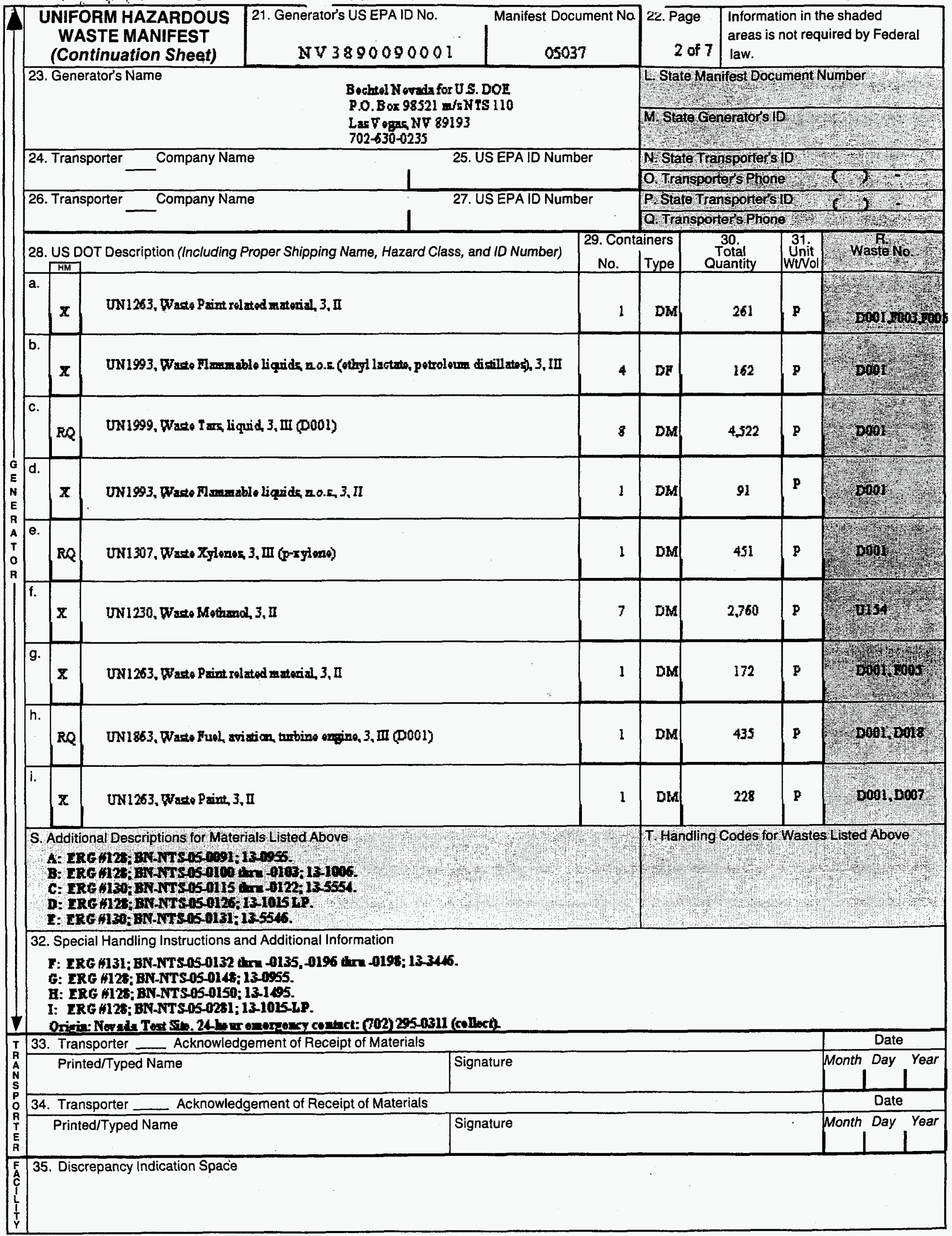




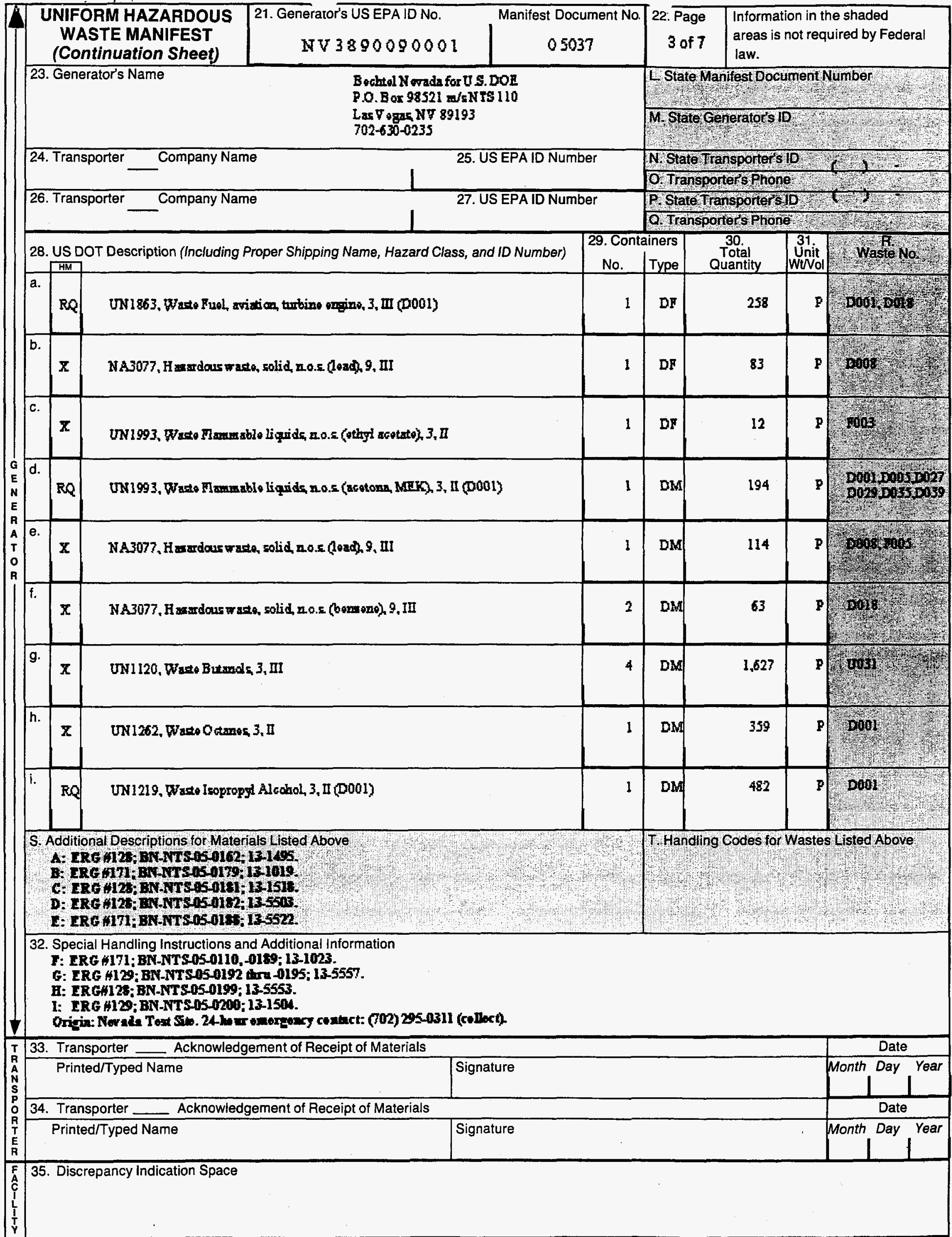




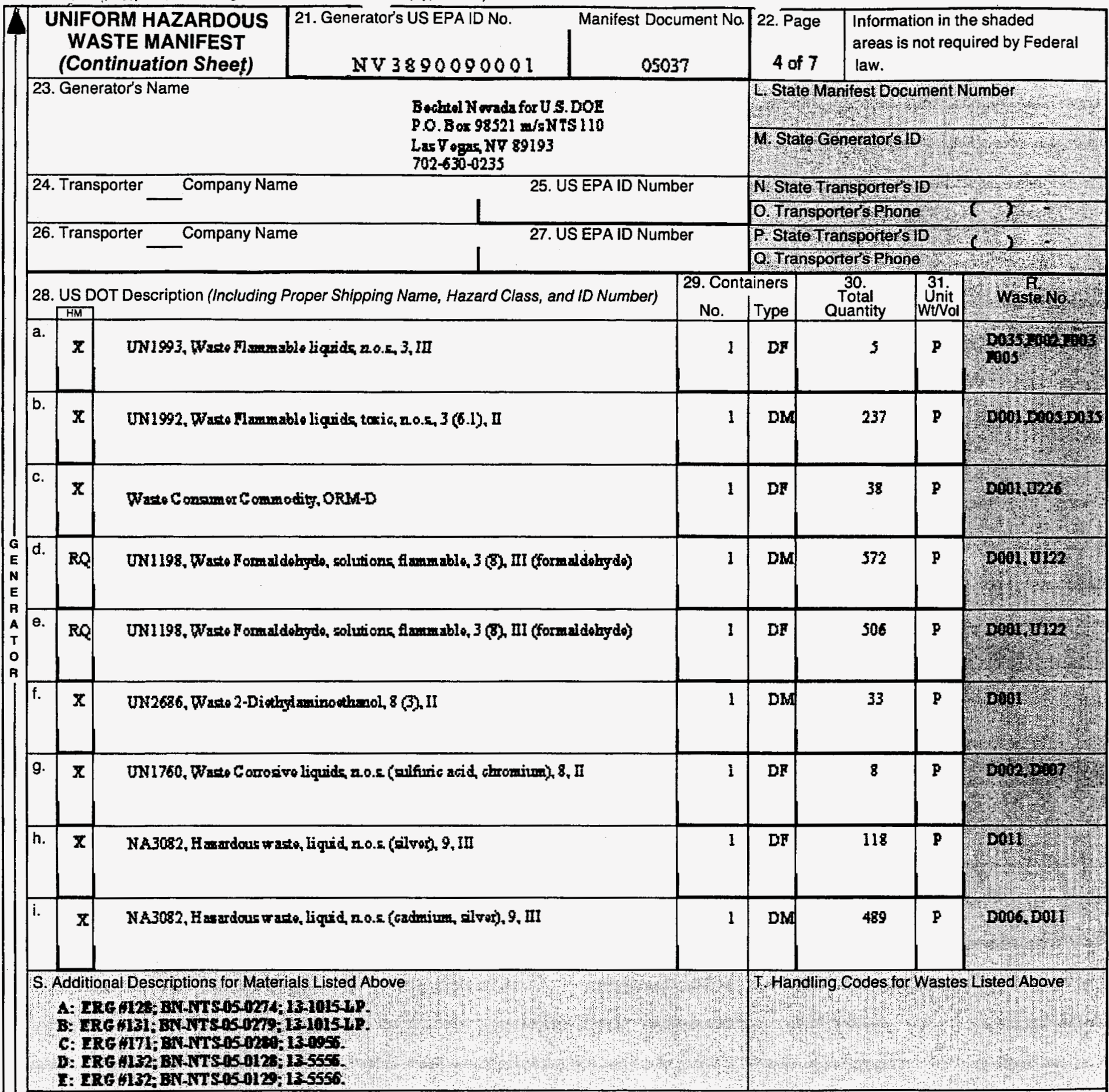

32. Special Handling Instructions and Additional Information
F: LRG 4132; BN-NTS-050130; 13-5544.
G: ERG 4154; BN-NTSOS-0167; 135015
H: LR EA171; BN-NTSL050092; 13-1013.
I: IRG A171; BN-NTS050093; $13-1024$.

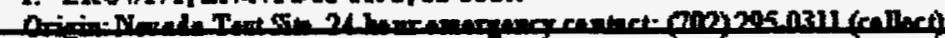

\begin{tabular}{|c|c|c|}
\hline 33. Transporter __ AC & & Date \\
\hline Printed/Typed Name & Signature & Month Day Year \\
\hline \multicolumn{2}{|c|}{ 34. Transporter ___ Acknowledgement of Receipt of Materials } & Date \\
\hline Printed/Typed Name & Signature & Month Day \\
\hline 35. Discrepancy Indicatio & & \\
\hline
\end{tabular}


Please print or type. (Form designed for use on elite ) itch) typewriter.)

Form Approved. OMB No. 2050-0039.

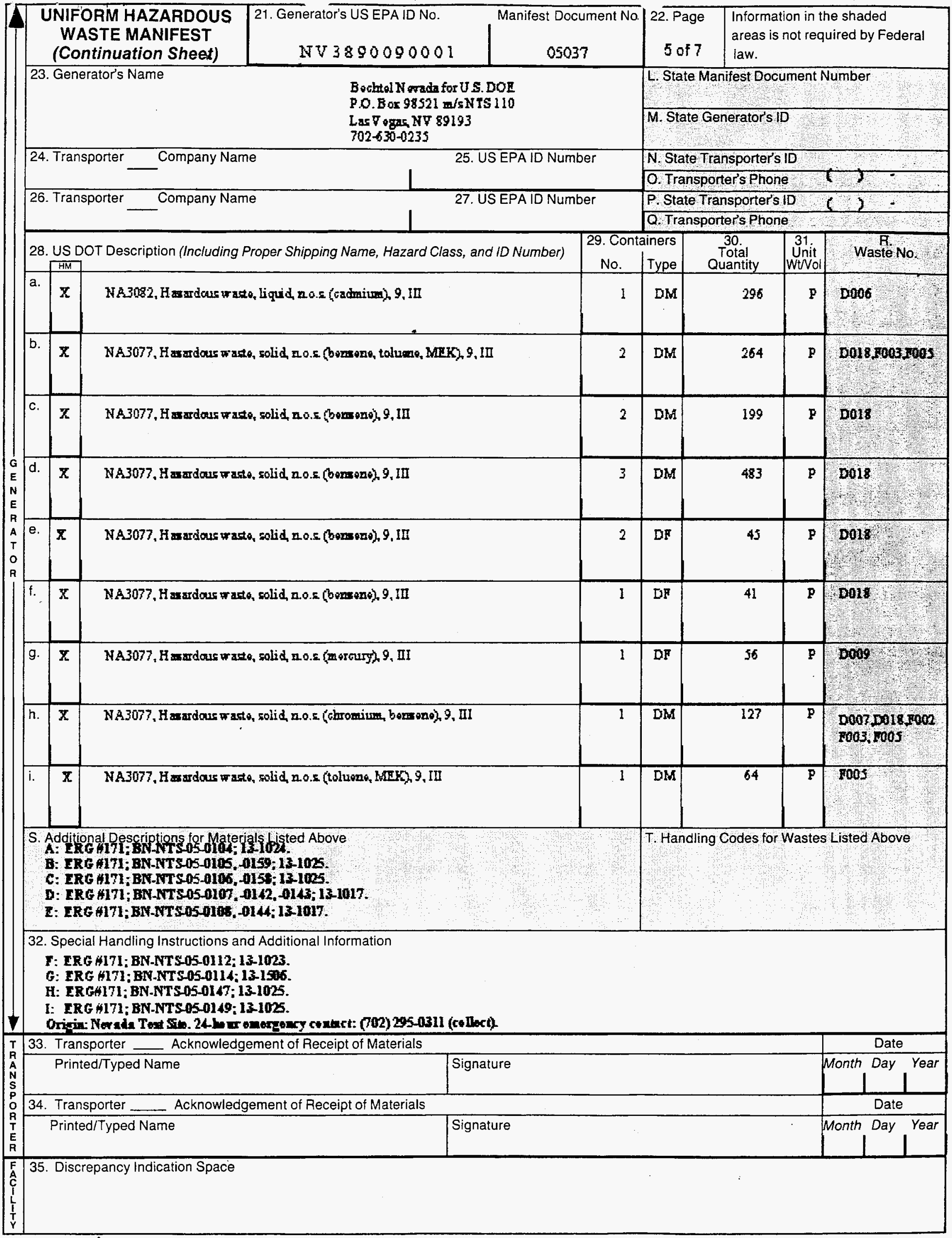


Pleáse print or type. (Form designed for use on elite litch) typewriter.)

Form Approved. OMB No. 2050-0039.

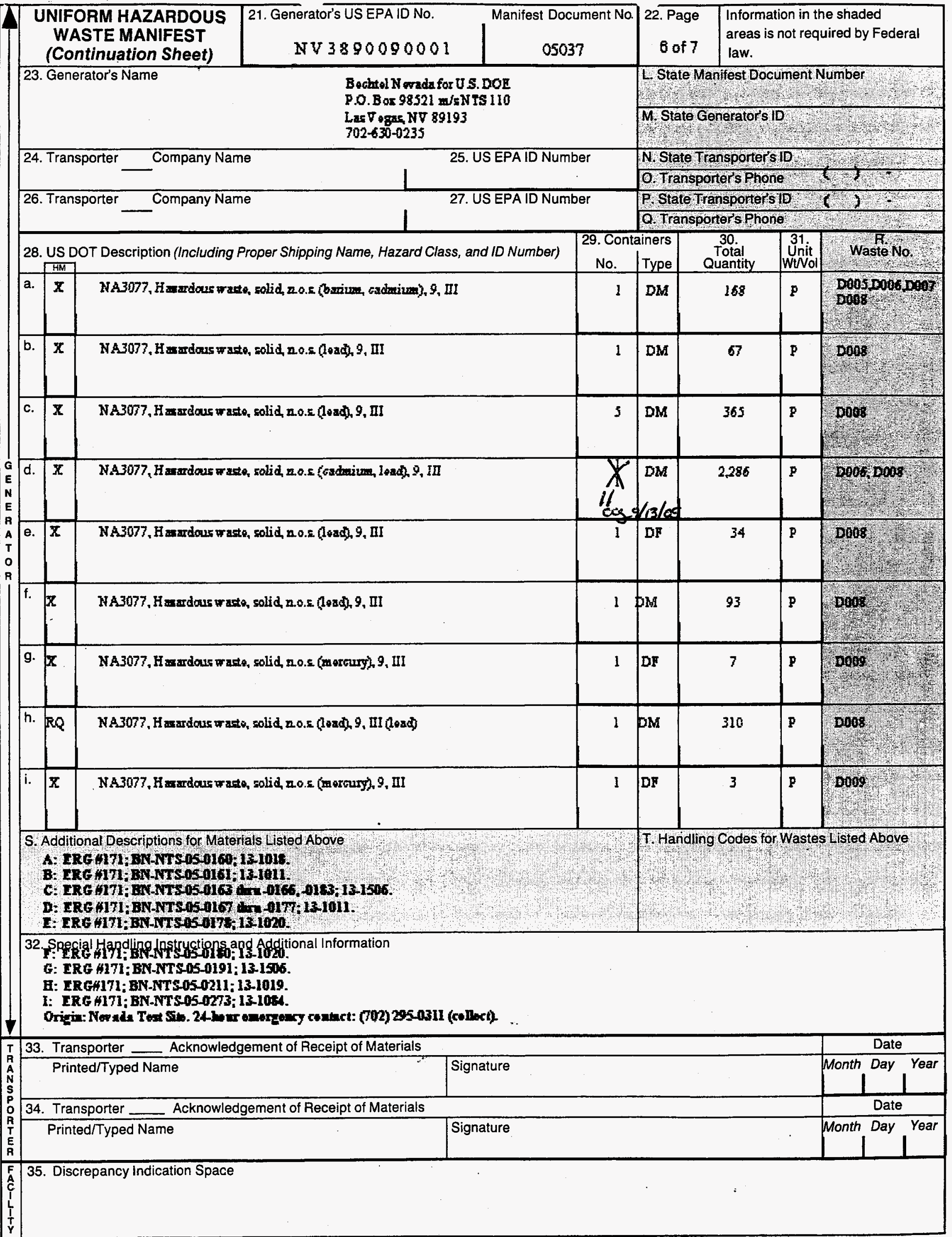




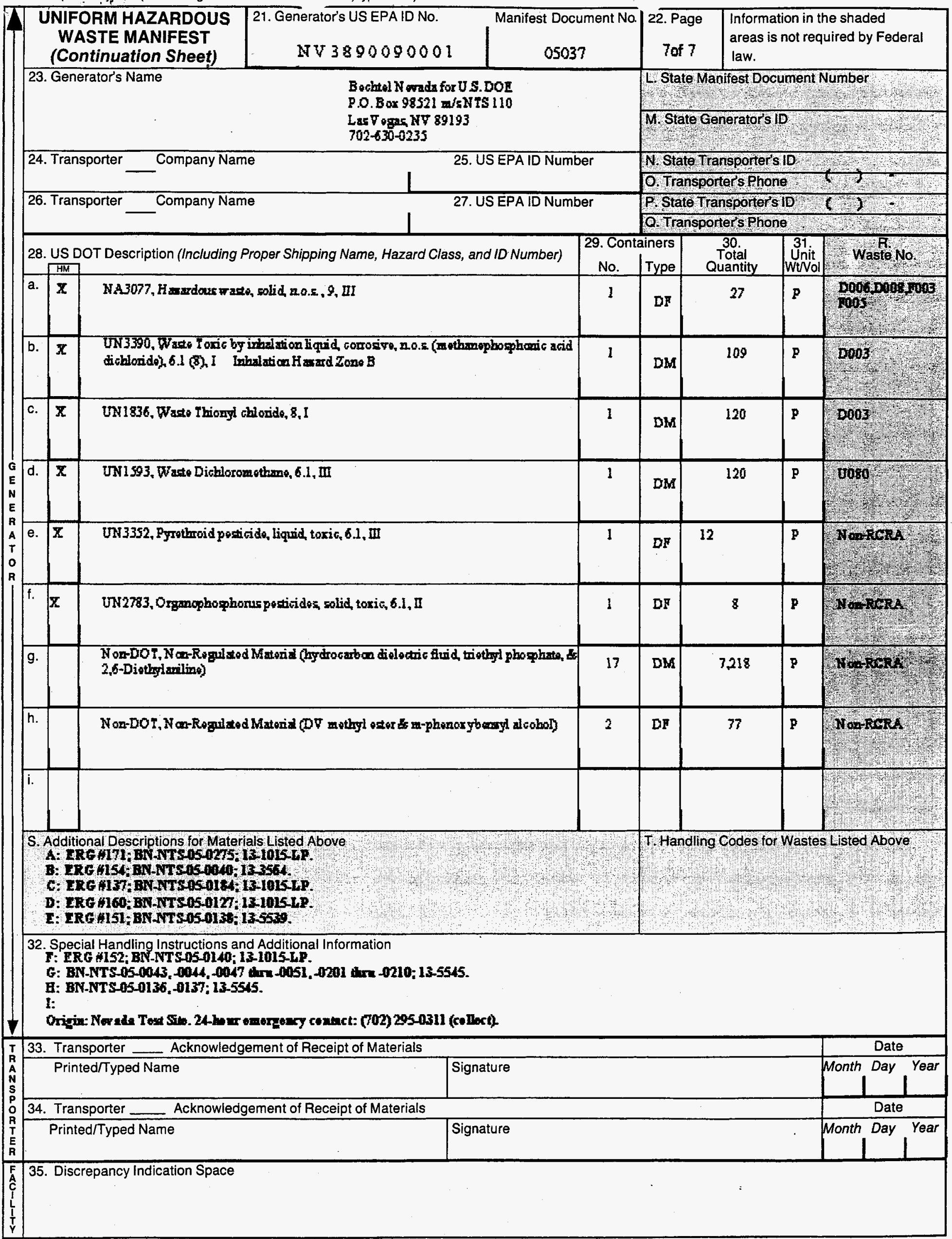


Carrier No.

Page 1 of 1
FINCHEM Company (Name of carrier)

(SCAC)

Date BecriteI Hengda for US DUE

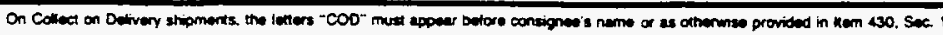

TO:

FUNCHEM Campany, inc

6133 Edith Blvd, NE

Consignee

Abuquerque, Nhy

Zip Code

87107

\begin{tabular}{|c|c|}
\hline $\begin{array}{l}\text { FaON: } \\
\text { Shipper }\end{array}$ & $\begin{array}{l}\text { Berite Ner ada for US DUE } \\
\text { Atm: C. Carlos Gonales }\end{array}$ \\
\hline Streot & $\begin{array}{l}\text { P.O. Bor } 58521, \text { msNTSTlo } \\
\text { Las V egas, Nex ada } 89193-8521\end{array}$ \\
\hline City & Physical Locatior state Jevada Teto Site \\
\hline
\end{tabular}

City

Slate

Vehicle

Route

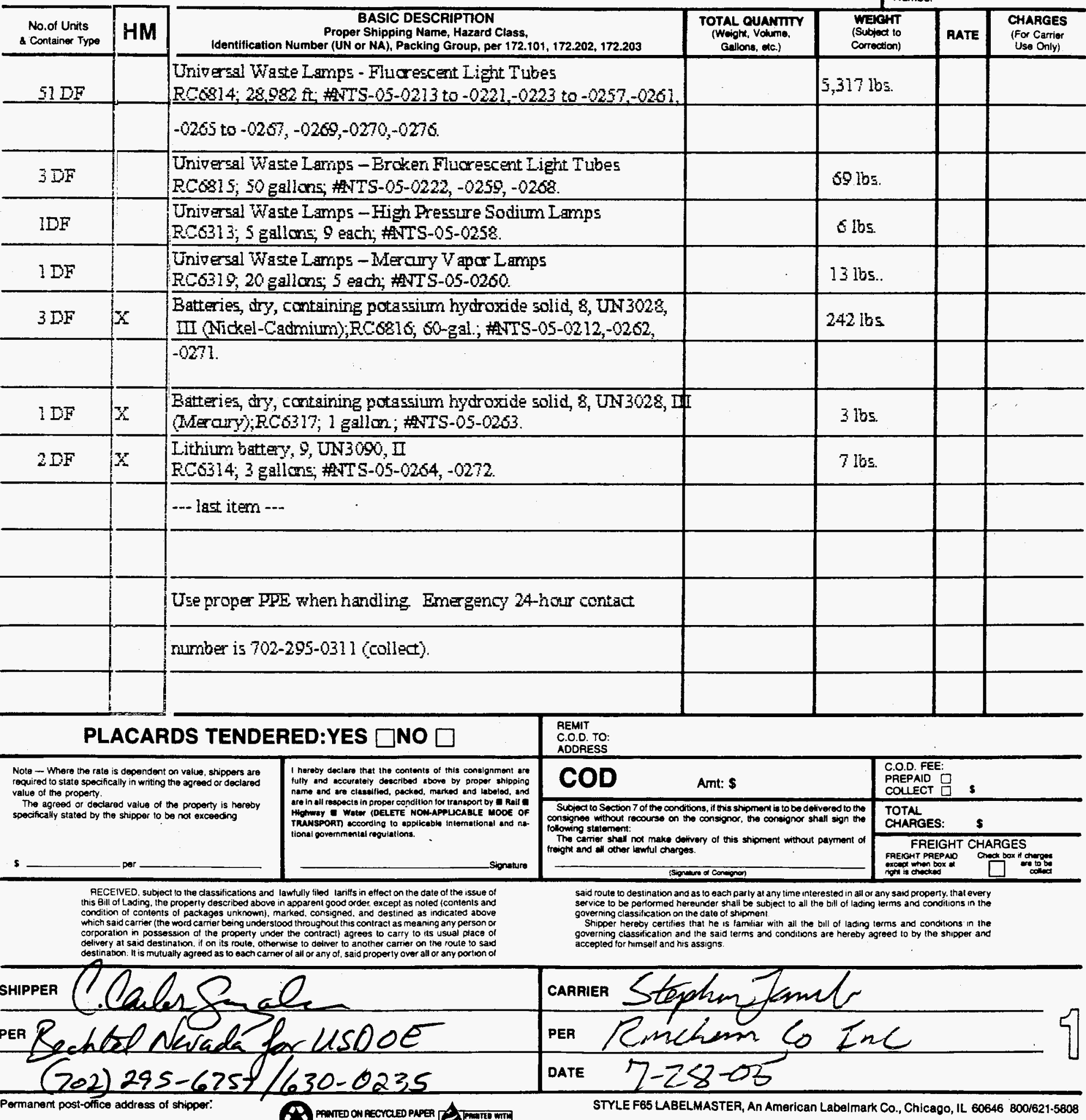




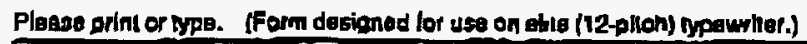

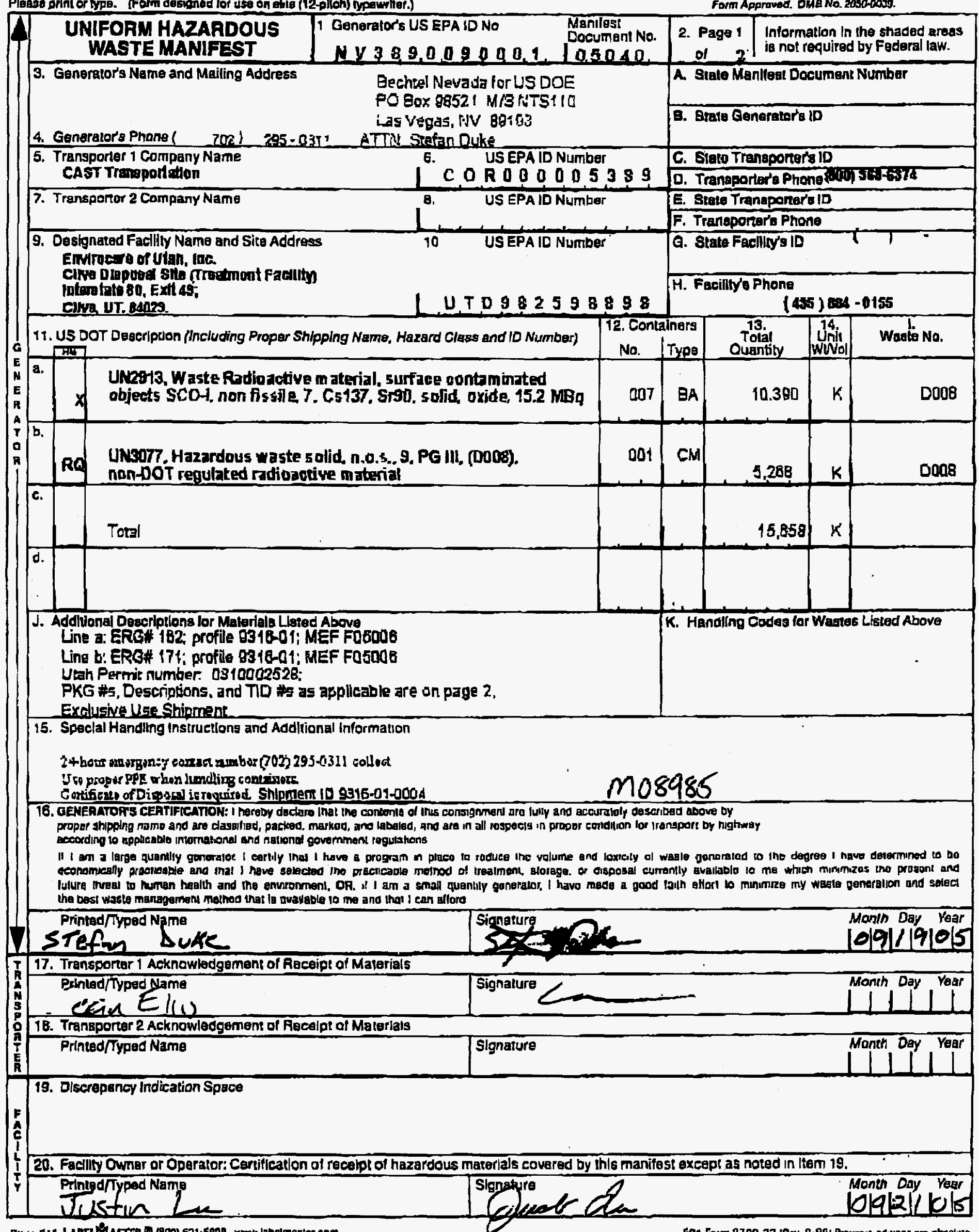


UNIFORM HAZARDOUS WASTE MANIFEST

3. Generator's Name and Mailing Address

4. Generalor's Phonel and

5. Transporter 1 Company Name CAST Traneportalion

7. Transporter 2 Company Name

9. Designaled Facilly Name and Bite Addrass Envifoer fo of uten, inc.

Give olapoas Gik (Treatrient Facindy interatalo 80, Exit 4t:

cina UIT RAMre.
2. Page 1 Inlormation in the shaded areas Document No.
2. Page 2 is not requlred by Federal law.

\section{A. Stare Manileat Document Number}

Gechtel Nevada for US DOE

PO Box ga521 MIS NTS1tO

Las Vegas, NV 89:63

\section{5 . 0.51 ATTh. Stafan Duk}

Siofan Duks

9. State Generator's ID

C. State Transponer's ID

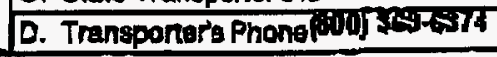

E. Stale Trangporters ID

F. Trenepontere Phone

G. Stape Fachlin's ID

H. Facll'ty's Phone

UTD932598B98

(45) 8 - 0455

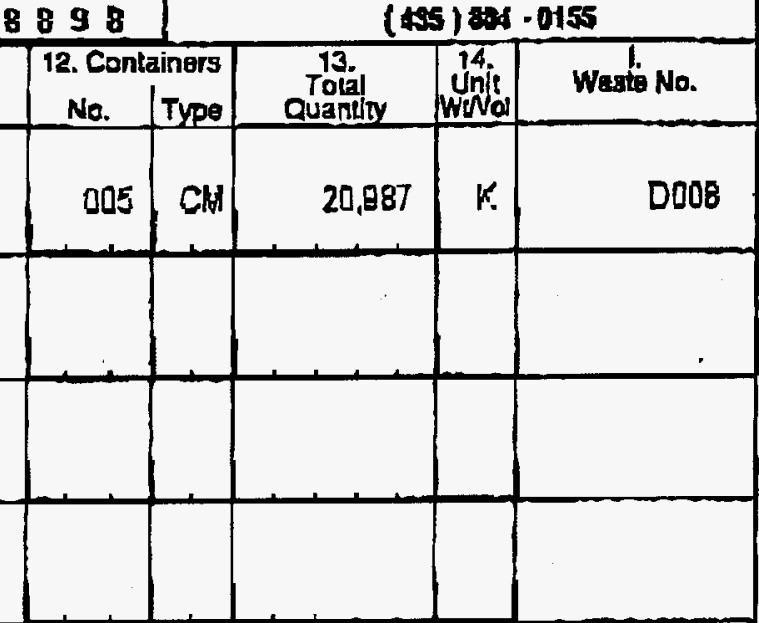

. K. Handling Codes for Wastes Latad Above

J. Addilional Descriptions for Materiala Liated Above

UNRA77, Hazardaus wa ste solid. n.0.5., 8, PG III, (DOO8).

11. US DOT Descriplion (Ineluding Proper Shipping Name, Hazerd Class and ID Number)

\begin{tabular}{|lll}
\hline a. & & \\
& &
\end{tabular}

b.

a.

c.

Line a: ERG\# 171; profile 9316-Q1; MEF F05000

Uah Permit number, 0310002528.

PKO W, Descripdons, and $7 D$ \&as as applioable are on page 2,

15. Speclal Handing Instruetions and Additional Intormation

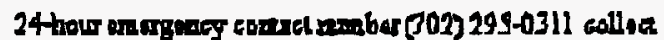

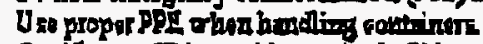

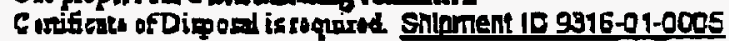

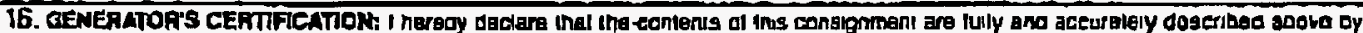

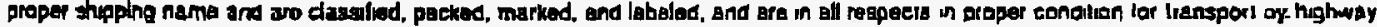

acuording lo apduczole intornatianal and namonal gavernmant ragulatians

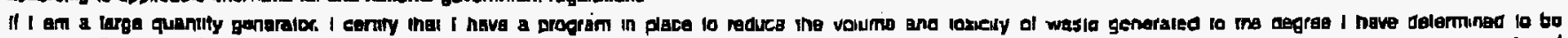

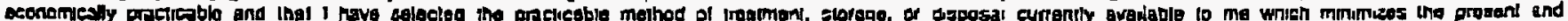

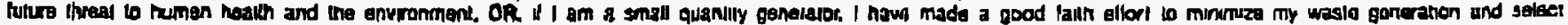
the bast waste managenerd moshod that is available to me and inat I can aflord.

Printed/Typed Name

Srefin Duke

T 17. Traneporter 1 Acknowtedgement of feceipt of Materials

Printad/Typed Name

H Pavald D Mathias

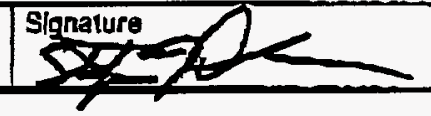

Wonth Day Year

18. Transponer 2 Acknowledgement of Recelpt of Materlals

Princed/lypod Namo

19. Discrepancy Indiostion Space

i.

20. Facilly Owner or Operator: Cerriflcation of receipt of hazardous materials covered by this manilest except as noted in Item 19.

PrincedTyped Namo J. Gordhar

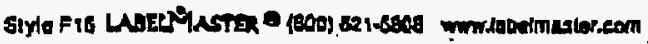

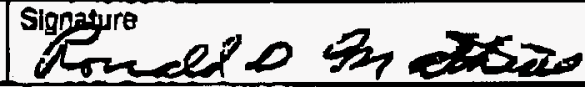

Slgnature 
Fab-08-2006 12:07pm From-WASTE OPERATIONS

\section{NON-HAZARDOUS WASTE MANIFEST}

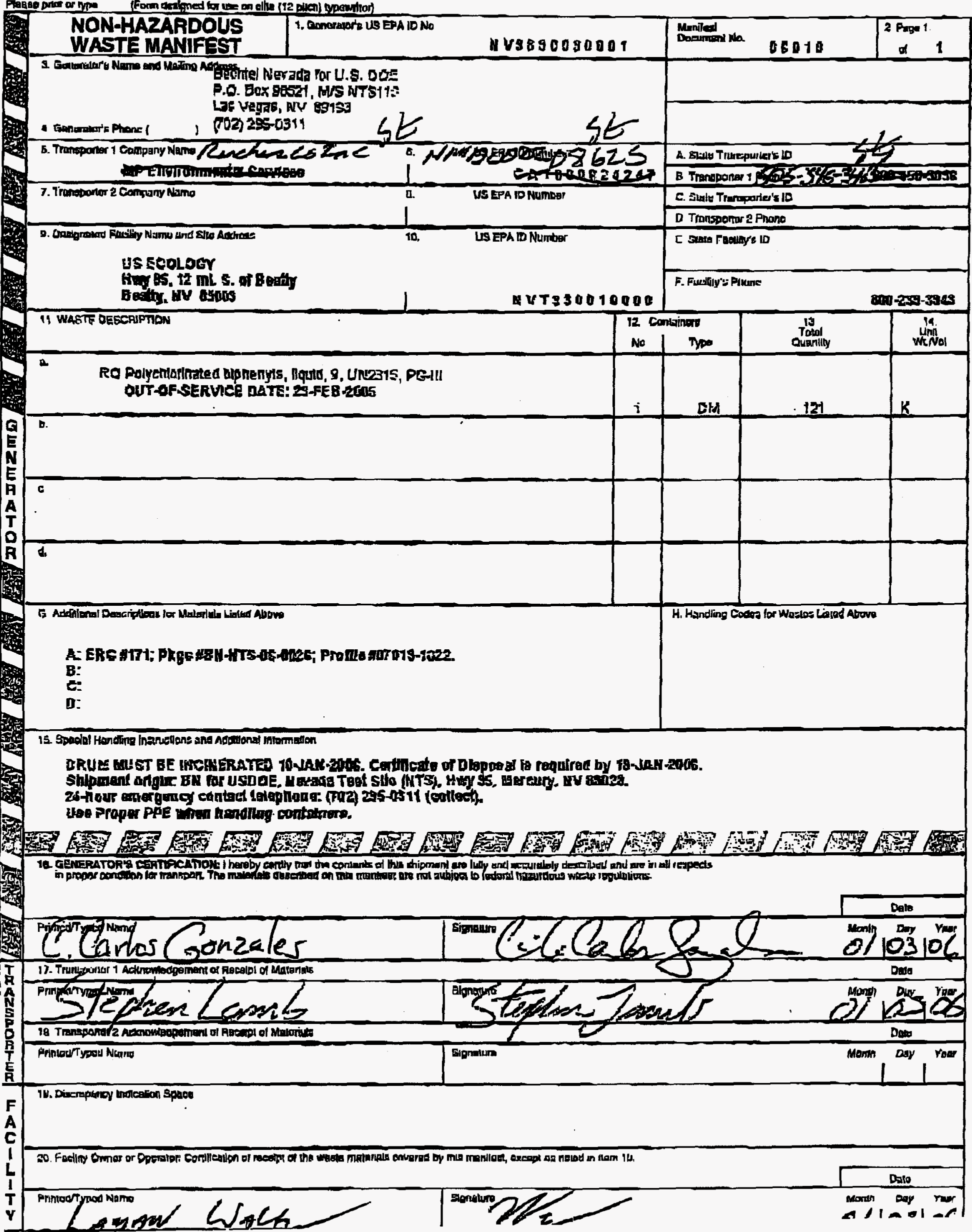




\section{Certificate of Recaipt and DIsposal of PCE Wasfe}

\section{Disposal Facility}

US Eealogy line.

P.D. Bax 578

Bantty, NV. 89003

\section{EPA identivication Number}

NVT330010000

\section{Disposal Certification}

\section{Generatar Neme: Bechtel Nevada for US DOE}

I HEREBY CERTIFY THAT PCE WASTE MATERIAL LISTED ON MANIFEST NO: AND ANY ATTACHMENTS WAS;

[X] Raceived at the US Ecology, Inc. Beatty, Nevada facility on $1 / 3 / 200 B$

I i Landfilled al the US Eoology, inc., Beatty, Nevada faeility on

1 ] Stored pending shipment for Off-Slle Treatment/neineration

I. Shlpped for Incineration to Onyx P.A. on Manifest Na. 1- 55 Gal. Drum Lg. Caps (Line 1a.)

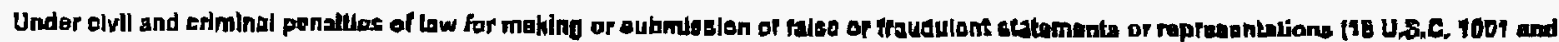

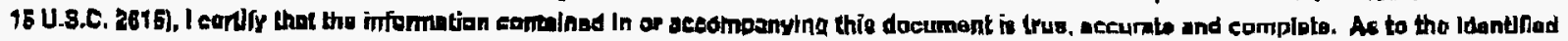

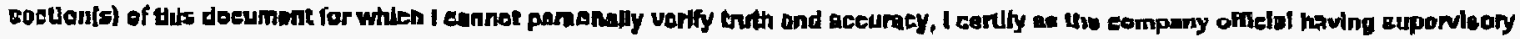

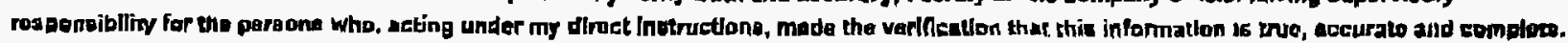

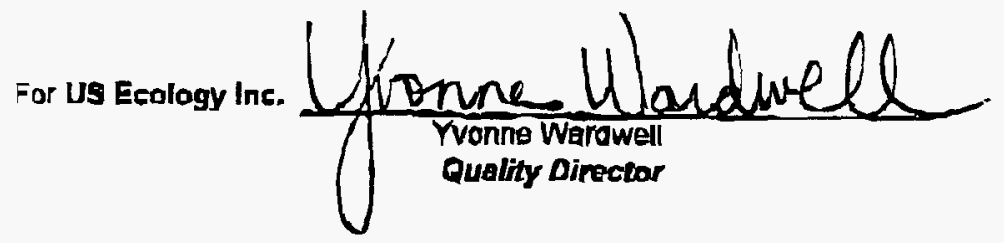

Data: 1/23/2006 
ENMRONMENTAL SERVICES, L.L.C.

Federal EPA ID: TXD000838896

State EPA ID: 50212-001

Highway 73

Port Arthur, TX 77643

(409) 736-2821

US ECOLOGY INC

ATTN: MANIFEST SECTION

NVT330010000

HWY 9511 MI SO OF BEATTY

BEATTY, NV 89003-0578

\section{CERTIFICATE OF DESTRUCTION}

Onyx Environmental Services, L.L.C. has recelved waste material from US ECOLOGY INC an 1/6/2006 as deseribed on [State Manifest or Unlform] Hazardous Waste Manifest number 0003441183, Onyx Environmental Services, L.L.C., hereby certifies that the above described material was inclnerated, and thereby destroyed, in accordance with the 40 CFR, part 761, as it pertains to the incineration of Poly-Chlorinated Biphenyl contaminated materials.

Sequence 1

Profile Number: CM2730

Onyx Tracking 1D: $\quad 588281-01$

Process Onnx Unlt ID Treatment Date Generator \#

$\begin{array}{llll}\text { INCINERATION } & 1 & 1 / 22 / 2006 & \text { OED10 }\end{array}$

Under civll and criminal penalties of law for the making or submission of false or fraudulent statements or representations (18 U.S.C. 1001 and 15 U.S.C. 2615), I certfy that the information contained in or accompanying this document is true, accurate, and complete. As to the identffed section(s) of this document for which I cannot personally verify truth and accuracy. I certify as the company official having supervisoly responsibility for the persons who, acting under my direct instructions, made the verification that thls information is true, accurate, and complete.

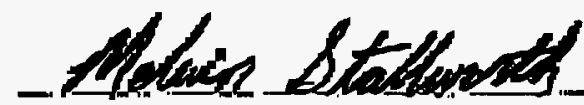

23-Jan-06 
Closure Report - CAU 115

Section: Appendix D

Revision: 0

Date: March 2006

THIS PAGE INTENTIONALLY LEFT BLANK 
Closure Report - CAU 115

Section: Appendix E

Revision: 0

Date: March 2006

\section{APPENDIX E}

\section{USE RESTRICTION DOCUMENTATION}


Closure Report - CAU 115

Section: Appendix E

Revision: 0

Date: March 2006

\section{THIS PAGE INTENTIONALLY LEFT BLANK}




\section{CAU Use Restriction Information}

CAU Number/Description: CAU 115: Area 25 Test Cell A Facility

Applicable CAS Numbers/Descriptions: CAS 25-41-04, Test Cell A Facility

Contact (organization/project): NNSA/NSO Industrial Sites Project Manager

Surveyed Area (UTM, Zone 11, NAD 27, meters):

\begin{tabular}{|c|c|c|}
\hline CORNER & NORTHING & EASTING \\
\hline \hline Northeast & $4,076,007.5$ & $566,256.7$ \\
\hline Southeast & $4,075,962.9$ & $566,256.7$ \\
\hline Southwest & $4,075,962.9$ & $566,230.3$ \\
\hline Northwest & $4,076,007.5$ & $566,230.3$ \\
\hline
\end{tabular}

Survey Date: $\underline{07 / 12 / 2005}$ Survey Method (GPS, etc): GPS

Site Monitoring Requirements: Visual Inspections

Required Frequency (quarterly, annually?): Annually

If Monitoring Has Started, Indicate last Completion Date: $\underline{\mathrm{N} / \mathrm{A}}$

\section{Use Restrictions}

The future use of any land related to this Corrective Action Unit (CAU), as described by the above surveyed location, is restricted from any DOE or Air Force activity that may alter or modify the containment control as approved by the state and identified in the CAU Closure Report or other CAU documentation unless appropriate concurrence is obtained in advance.

Comments: See the Closure Report for additional information on the condition of the site(s) and any monitoring and/or inspection requirements.

Submitted By: Sabine Cutis Date: $3-2-06$

cc with copy of survey map (paper and digital (dgn) formats):

CAU Files ( 2 copies) 


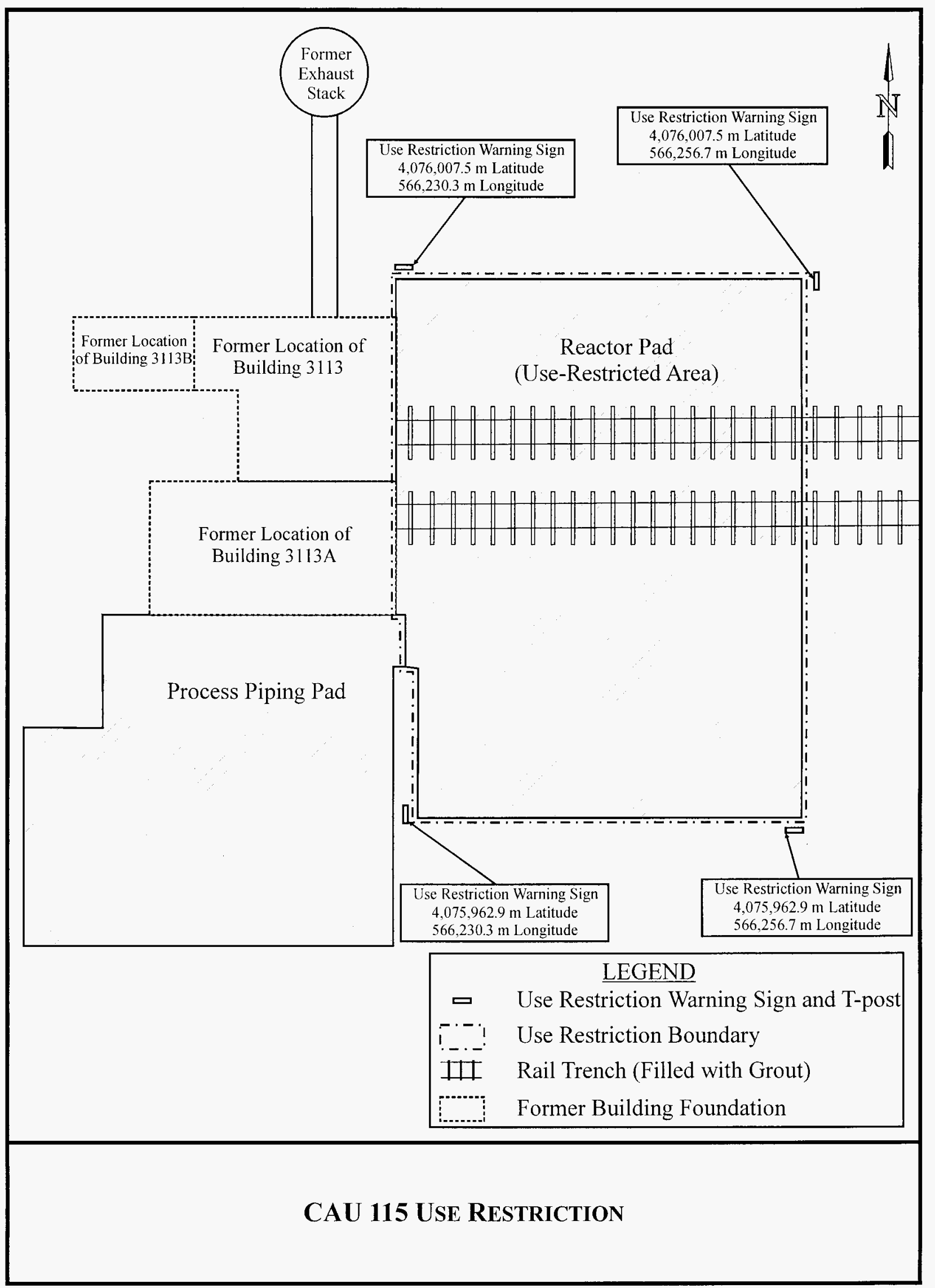


Closure Report - CAU 115

Section: Appendix F

Revision: 0

Date: March 2006

\section{APPENDIX F}

\section{SITE CLOSURE PHOTOGRAPHS}

F-1 
Closure Report - CAU 115

Section: Appendix F

Revision: 0

Date: March 2006

THIS PAGE INTENTIONALLY LEFT BLANK 
PHOTOGRAPH LOG

\begin{tabular}{|c|c|c|c|}
\hline $\begin{array}{l}\text { PHOTOGRAPH } \\
\text { NuMBER }\end{array}$ & DATE & PERSPECTIVE & DESCRIPTION \\
\hline 1 & $01 / 03 / 2005$ & Facing Northwest & Test Cell A Facility Before Closure Activities \\
\hline 2 & $01 / 03 / 2005$ & Facing Southeast & Test Cell A Facility Before Closure Activities \\
\hline 3 & $01 / 03 / 2005$ & Facing Southwest & Process Piping Pad Before Closure Activities \\
\hline 4 & $01 / 03 / 2005$ & Not Applicable & Cadmium Foil Covered Piping \\
\hline 5 & $02 / 24 / 2005$ & Not Applicable & Lead Brick Removal \\
\hline 6 & $03 / 08 / 2005$ & Not Applicable & Equipment Reservoir Fluid Sampling \\
\hline 7 & $04 / 20 / 2005$ & Facing East & Railroad Trench Grouting \\
\hline 8 & $04 / 20 / 2005$ & Facing East & Grouted Railroad Trenches \\
\hline 9 & $04 / 21 / 2005$ & Facing Northeast & $\begin{array}{l}\text { Radiologically Impacted Soil Removal near NW Corner of } \\
\text { Reactor Pad }\end{array}$ \\
\hline 10 & $06 / 02 / 2005$ & Facing West & Decontamination Activities on Roof \\
\hline 11 & $06 / 02 / 2005$ & Facing West & Exhaust Stack Demolition Debris \\
\hline 12 & $06 / 02 / 2005$ & Facing Southeast & Moveable Shed Demolition Debris \\
\hline 13 & $06 / 02 / 2005$ & Facing North & After Demolition of Building 3113B \\
\hline 14 & $06 / 02 / 2005$ & Facing North & After Removal of Process Piping \\
\hline 15 & $06 / 06 / 2005$ & Facing East & Demolition of Building 3113A \\
\hline 16 & $06 / 07 / 2005$ & Facing North & Building 3113A Demolition Debris \\
\hline 17 & $06 / 07 / 2005$ & Facing East & Demolition of Building 3113 \\
\hline 18 & $06 / 15 / 2005$ & Facing East & Demolition of Building 3113 \\
\hline 19 & $06 / 15 / 2005$ & Facing Southeast & Demolition of Building 3113 \\
\hline 20 & $06 / 15 / 2005$ & Facing East & Building 3113 Demolition Debris \\
\hline 21 & $06 / 15 / 2005$ & Facing South & Shield Wall After Demolition of Building 3113 \\
\hline 22 & $06 / 15 / 2005$ & Facing Northwest & Shield Wall After Demolition of Building 3113 \\
\hline 23 & $06 / 21 / 2005$ & Facing Southwest & Drilling of Shield Wall for Emplacement of Explosives \\
\hline 24 & $06 / 23 / 2005$ & Facing West & Shield Wall Prepared for Explosive Demolition \\
\hline 25 & $06 / 23 / 2005$ & Facing West & Explosive Demolition of Shield Wall \\
\hline 26 & $06 / 29 / 2005$ & Not Applicable & Grouting of Penetrations \\
\hline 27 & 07/07/2005 & Facing North & Low-Level Waste Currently Staged Onsite \\
\hline 28 & $07 / 09 / 2005$ & Facing Northeast & Perlite Removal From Dewar \\
\hline 29 & $08 / 16 / 2005$ & Facing Southwest & Demolition of Dewar \\
\hline 30 & $08 / 22 / 2005$ & Facing East & Final State of Remaining Concrete Foundations \\
\hline 31 & $08 / 22 / 2005$ & Facing Southeast & $\begin{array}{l}\text { Radiological Posting and Use Restriction Warning Sign for } \\
\text { Reactor Pad }\end{array}$ \\
\hline
\end{tabular}


Closure Report - CAU 115

Section: Appendix F

Revision: 0

Date: March 2006

THIS PAGE INTENTIONALLY LEFT BLANK 


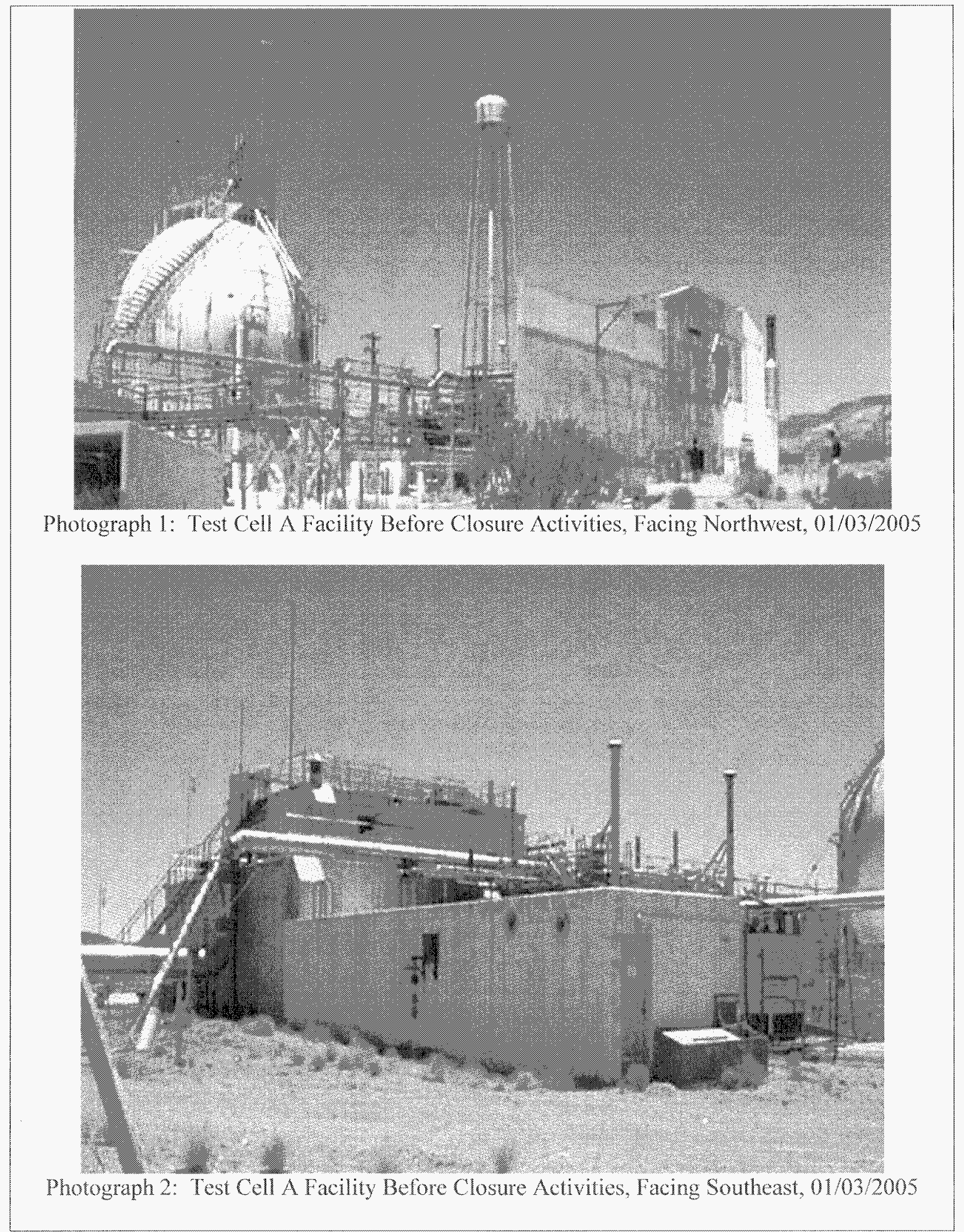




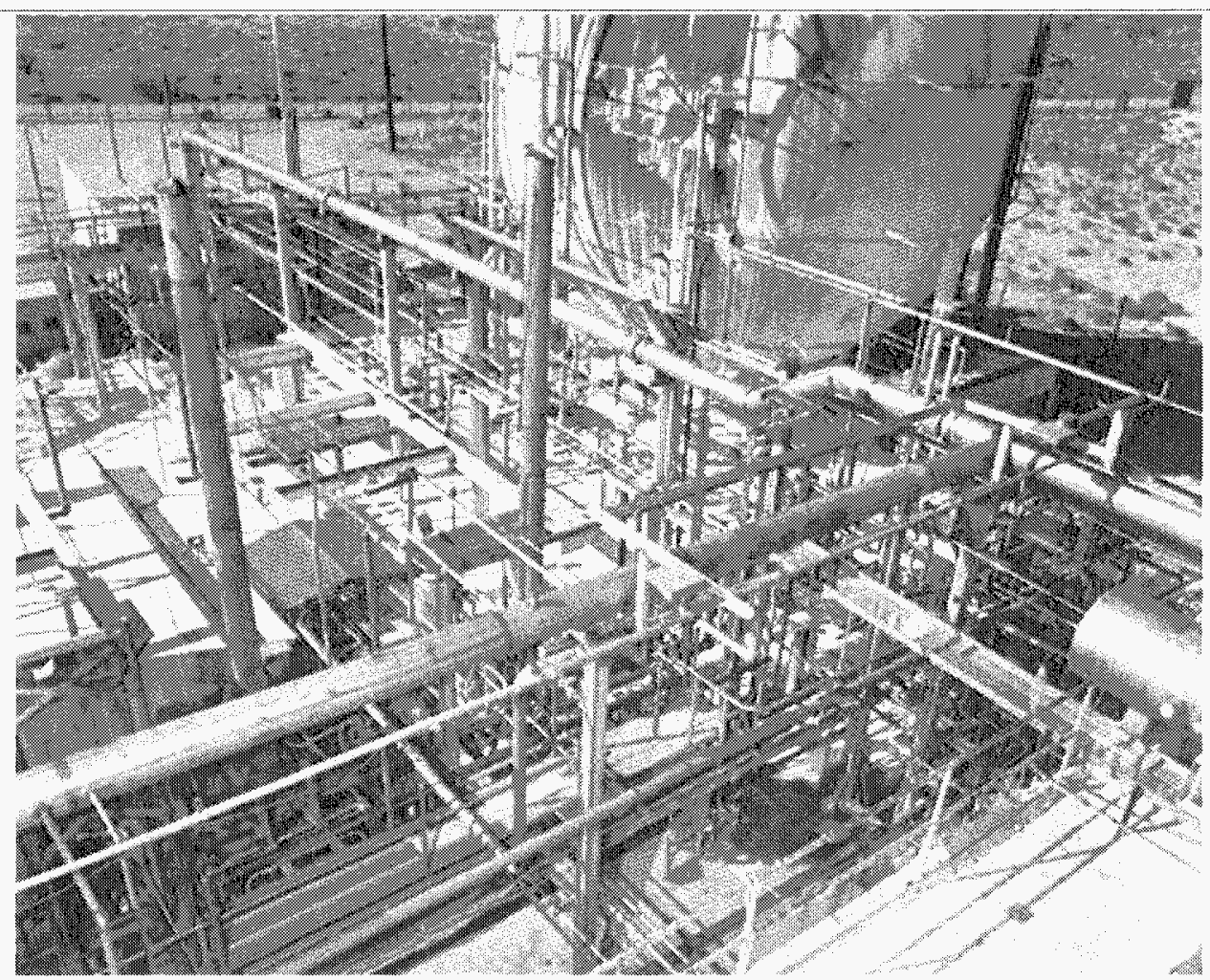

Photograph 3: Process Piping Pad Before Closure Activities, Facing Southwest, 01/03/2005

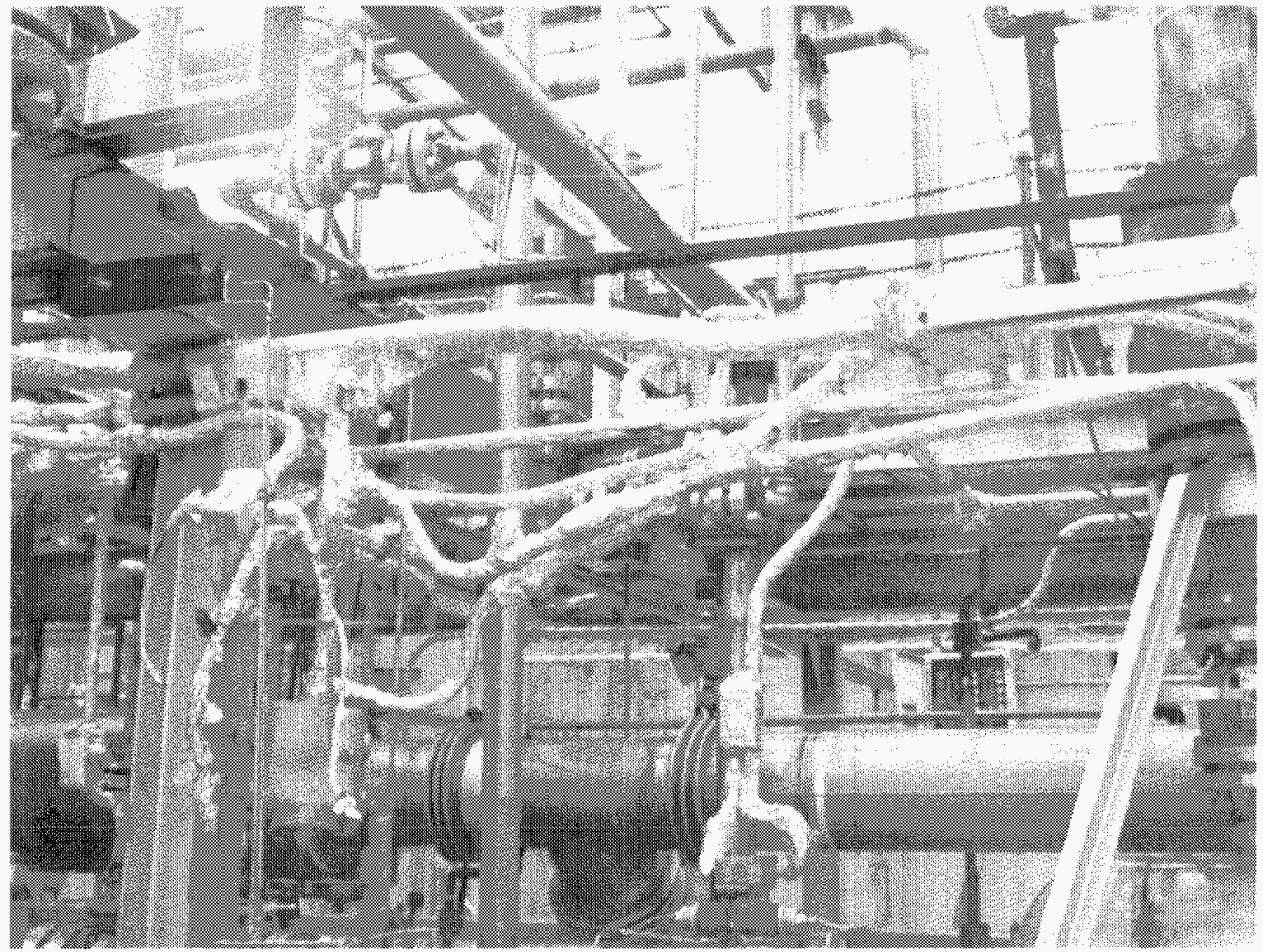

Photograph 4: Cadmium Foil Covered Piping, 01/03/2005 


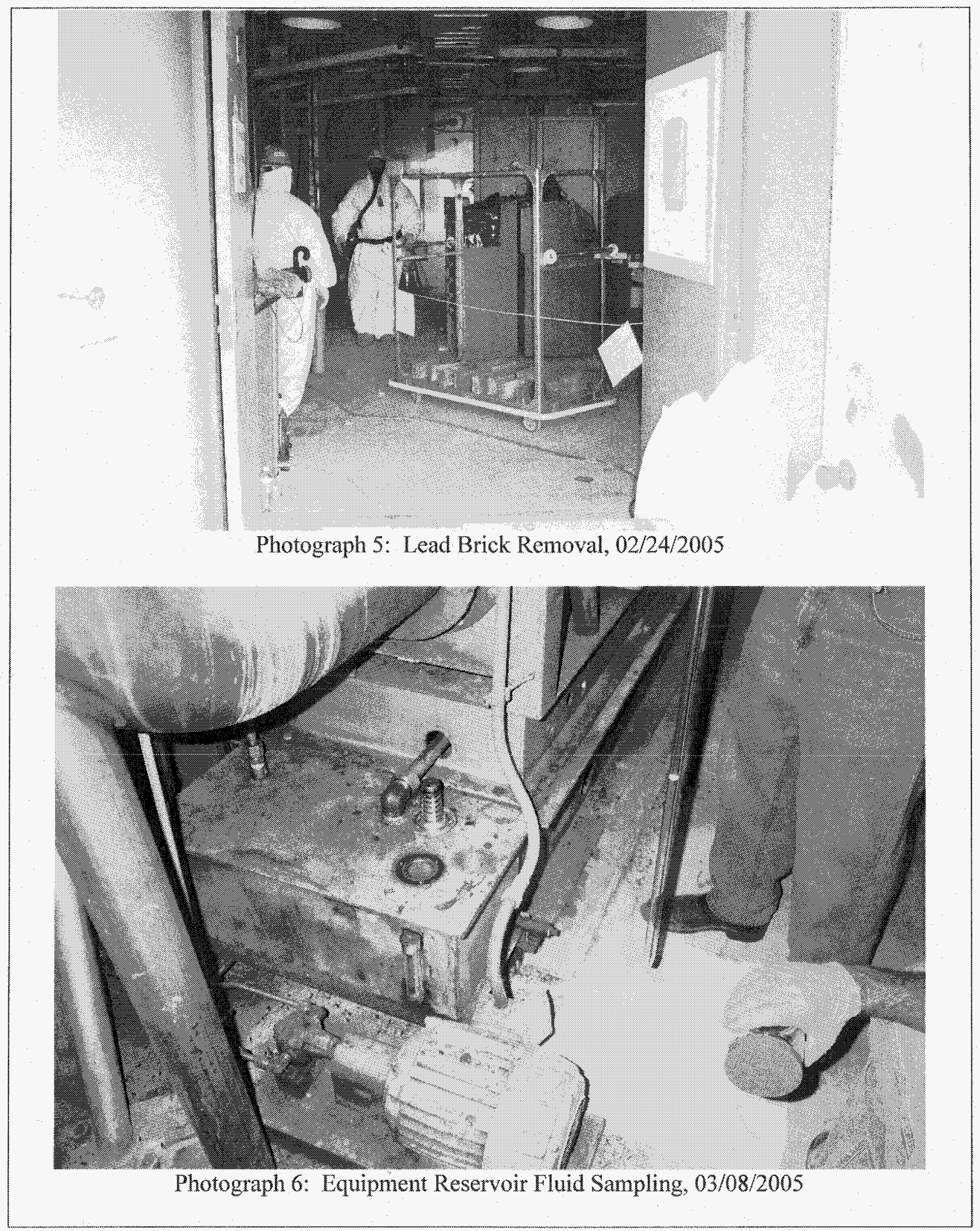




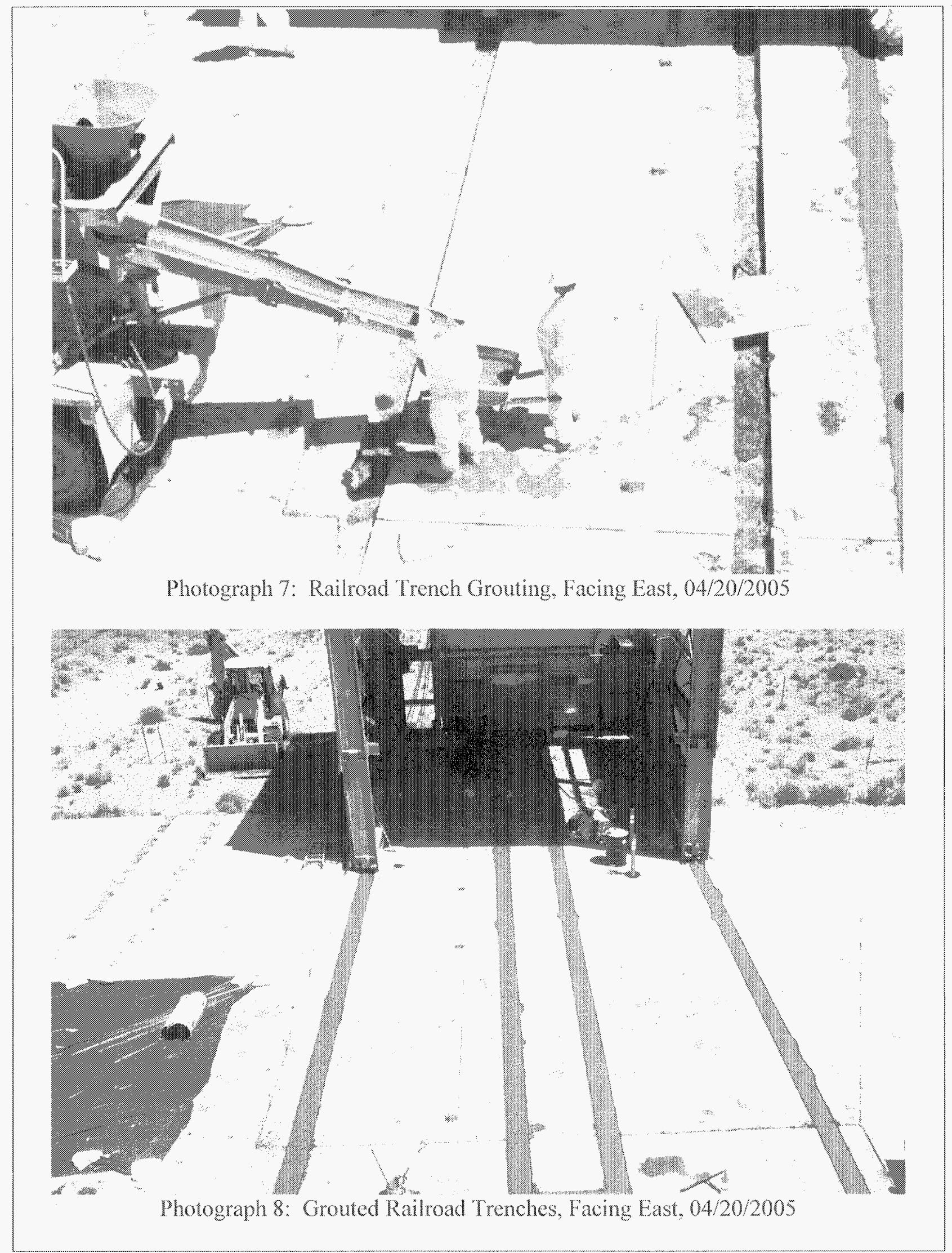




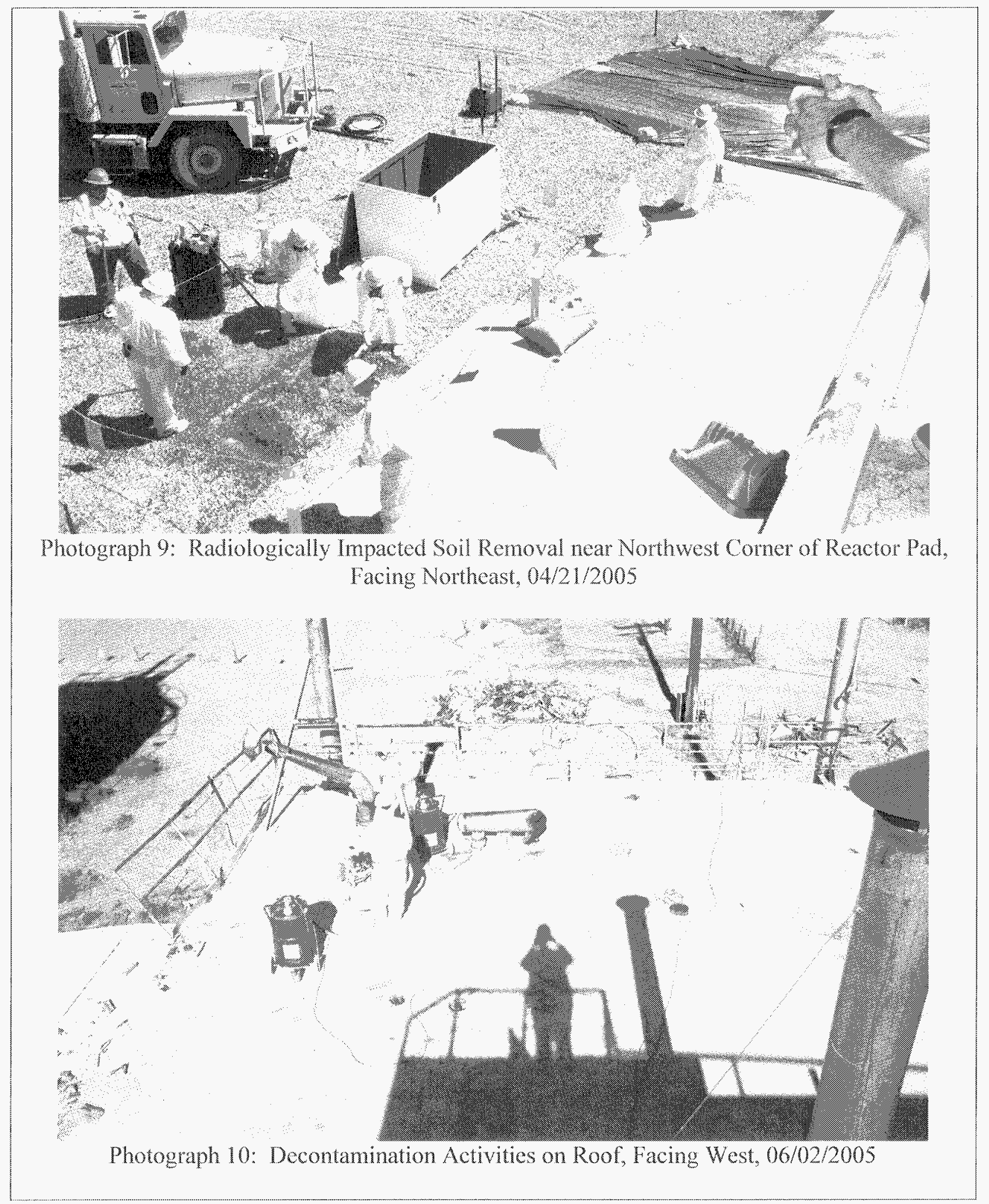




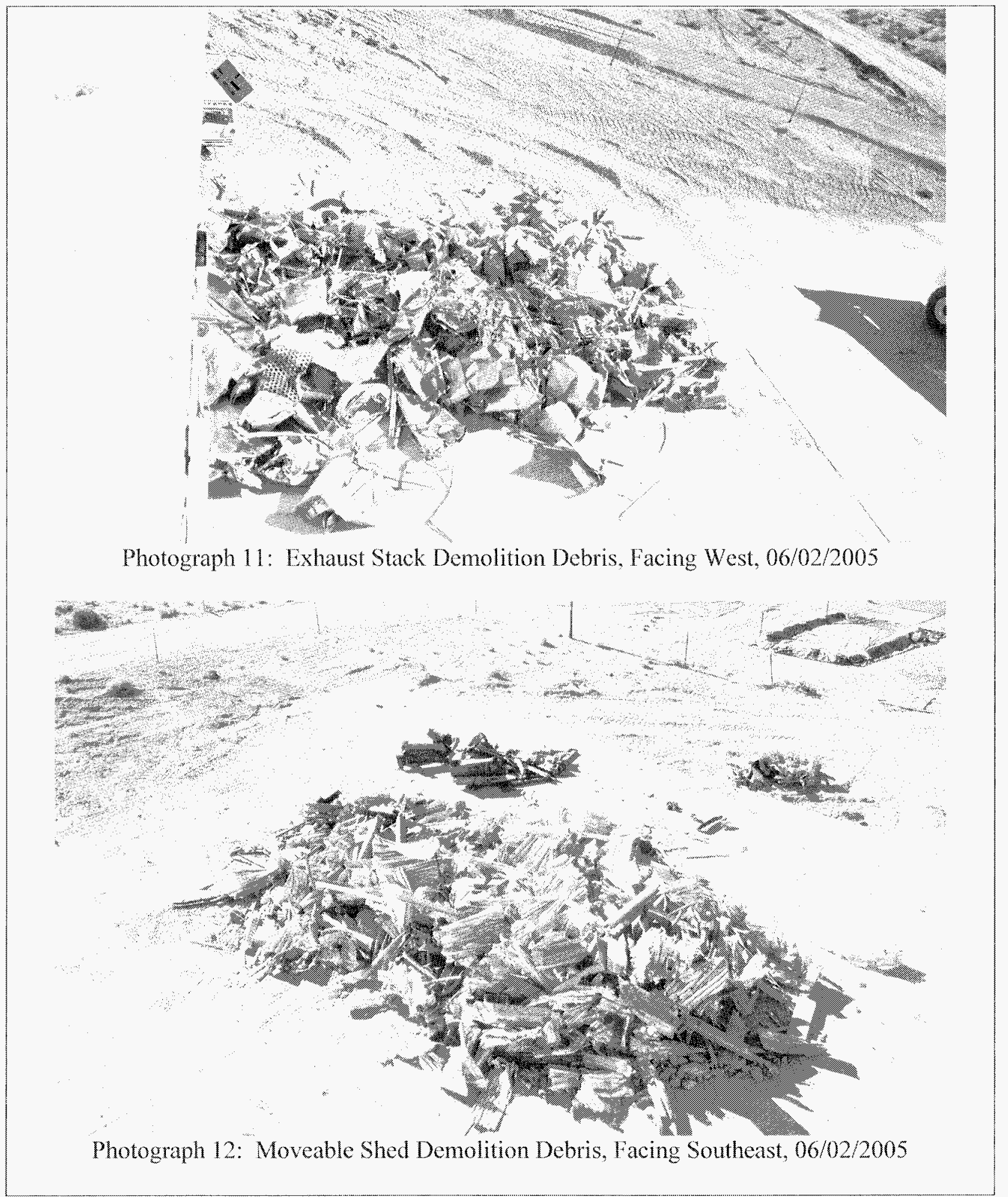




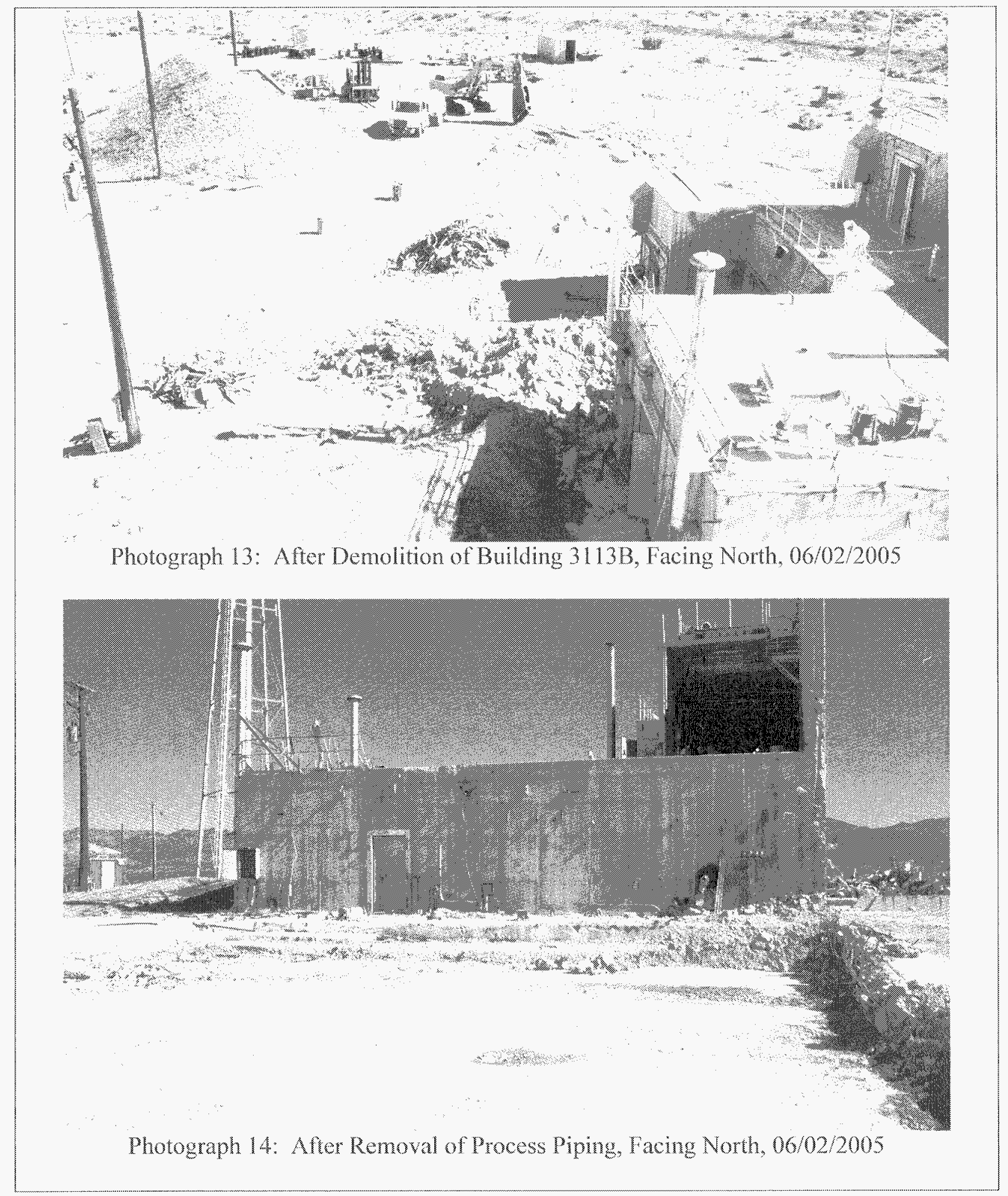




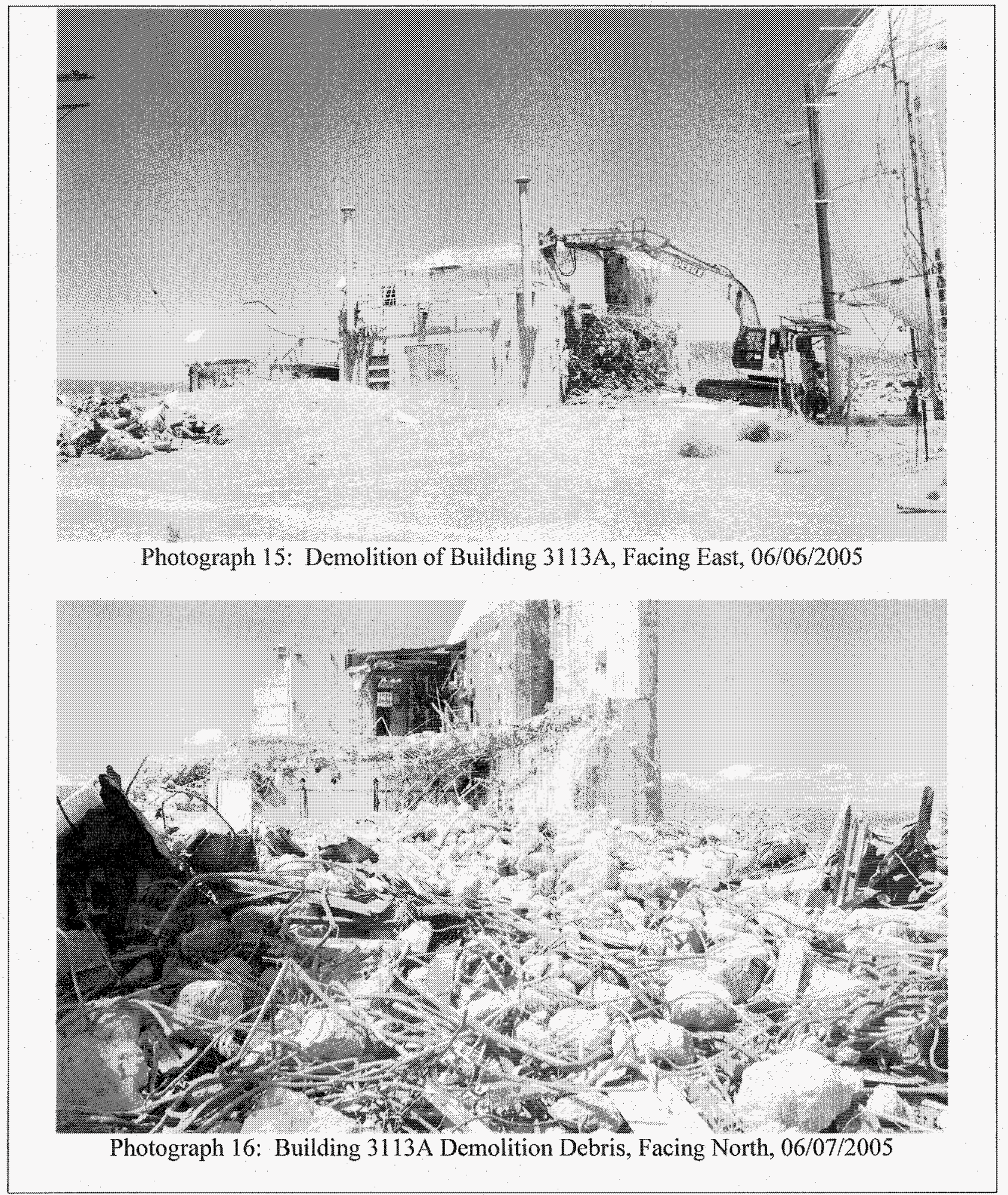




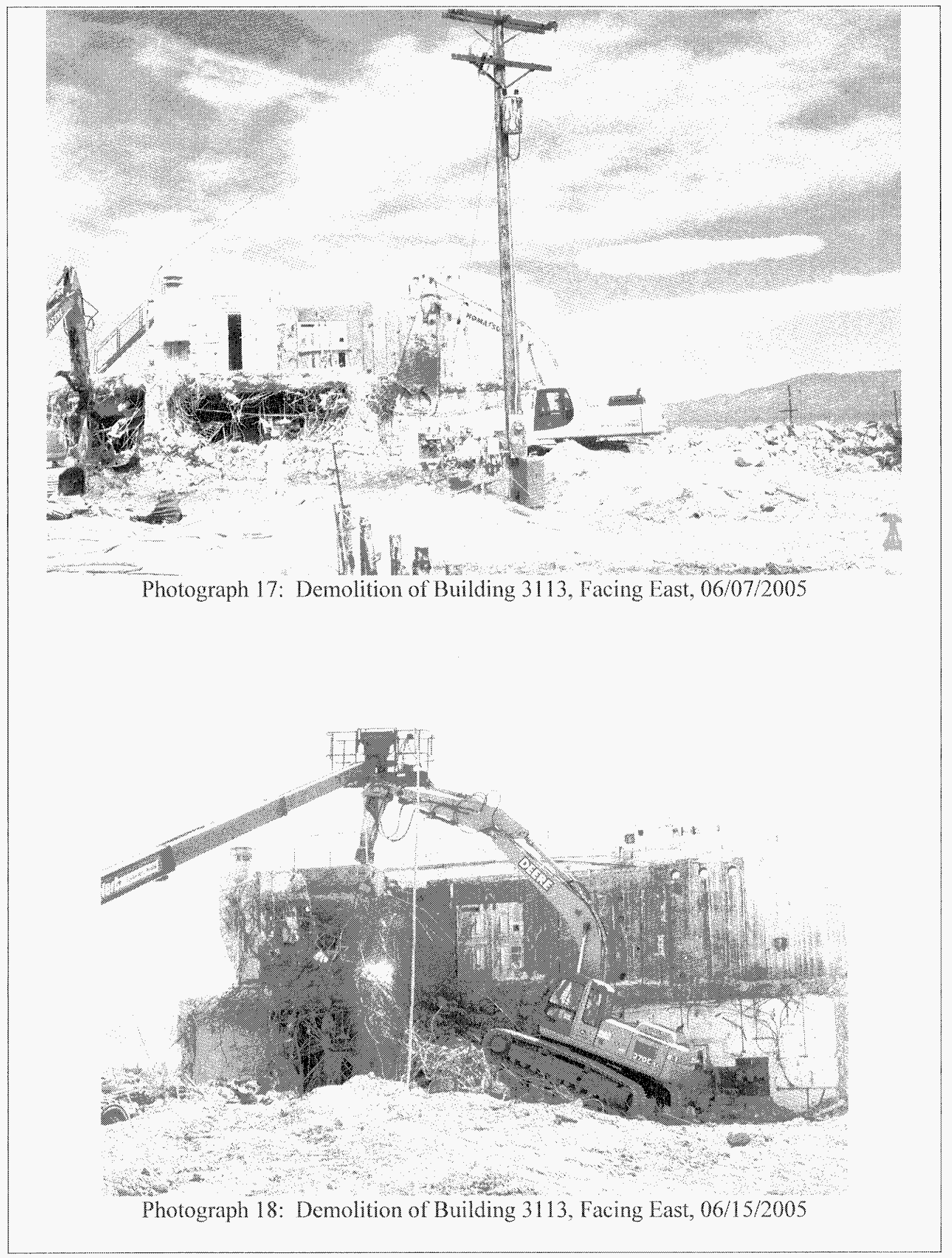




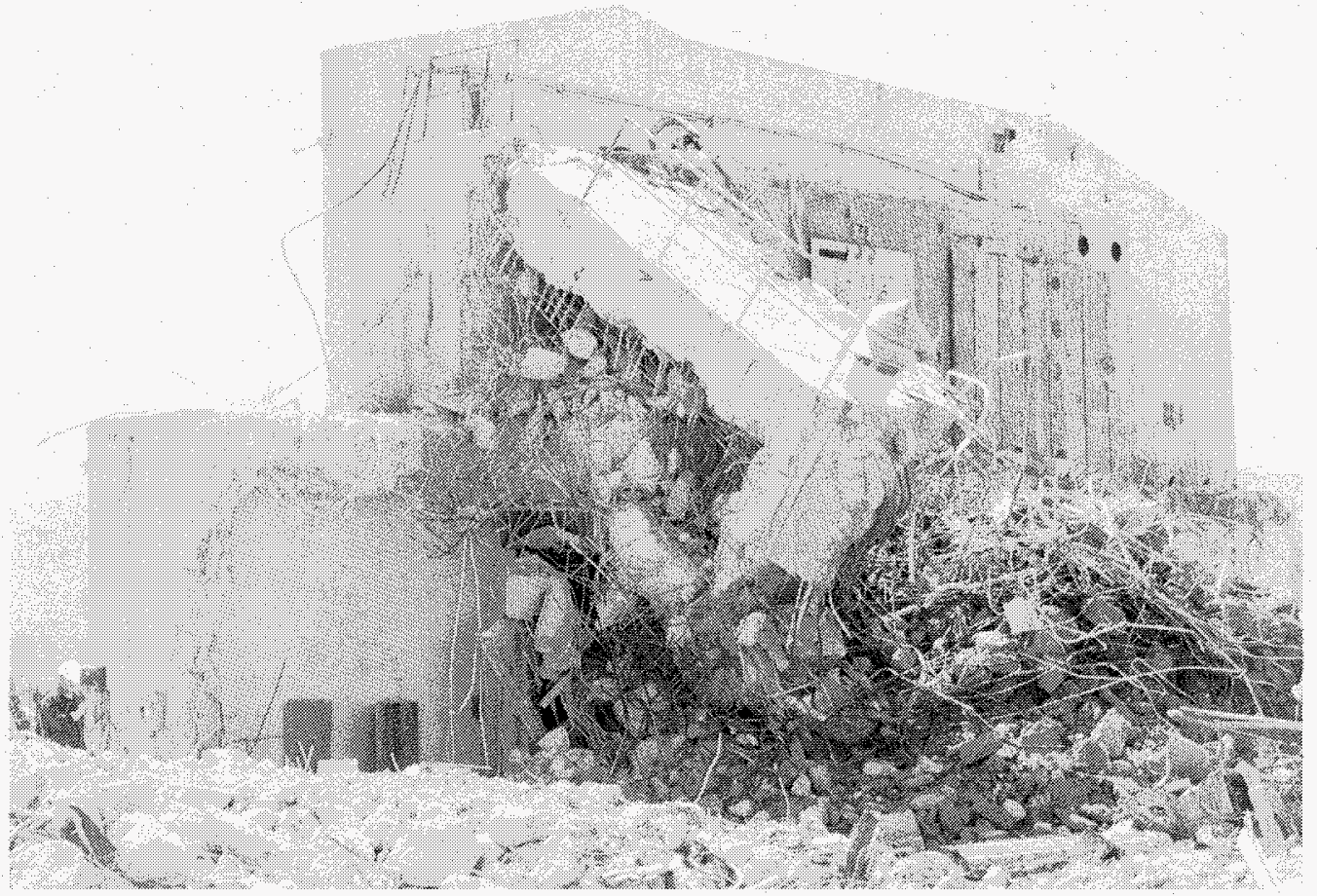

Photograph 19: Demolition of Building 3113, Facing Southeast, 06/15/2005

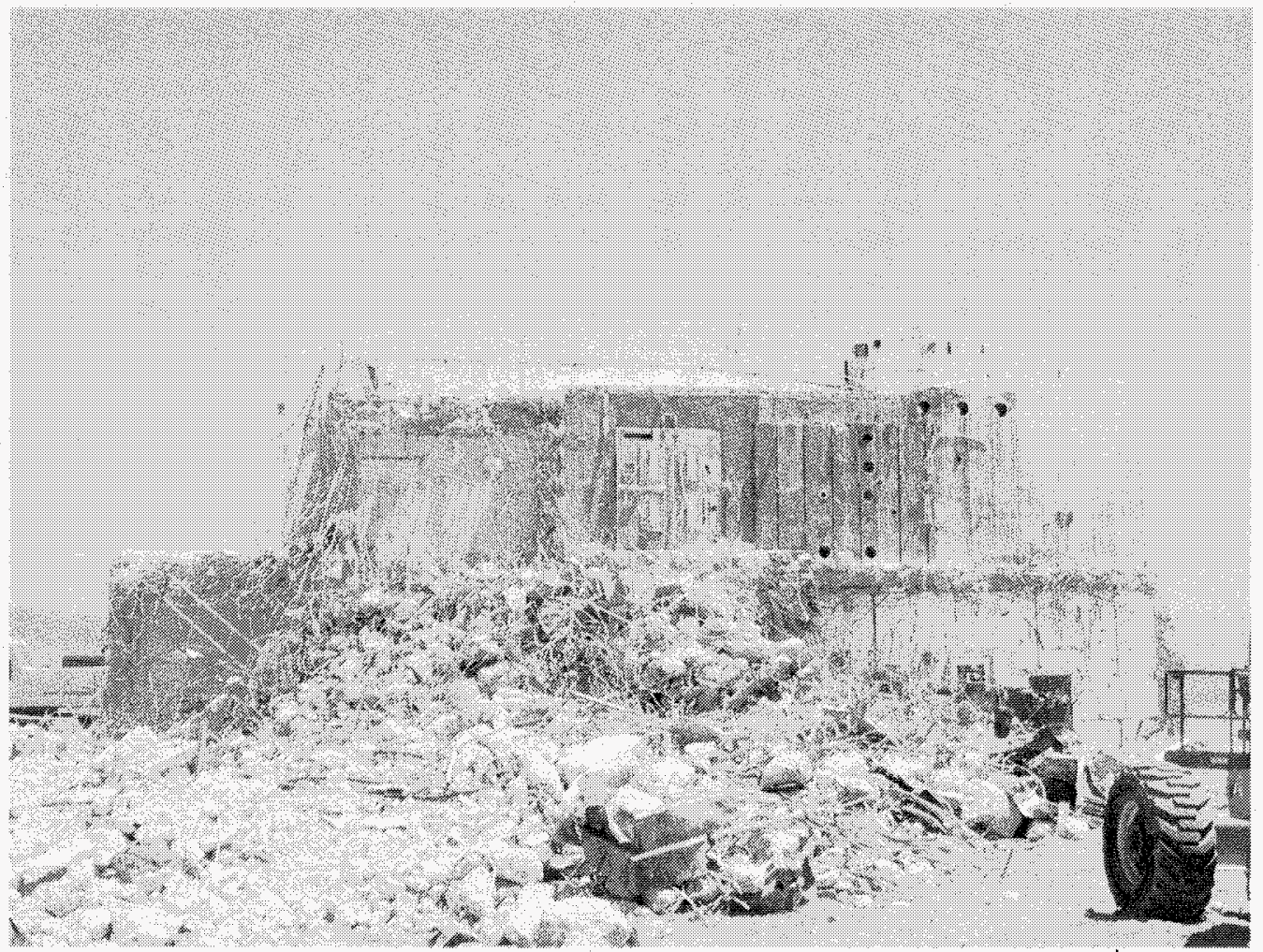

Photograph 20: Building 3113 Demolition Debris, Facing East, 06/15/2005 


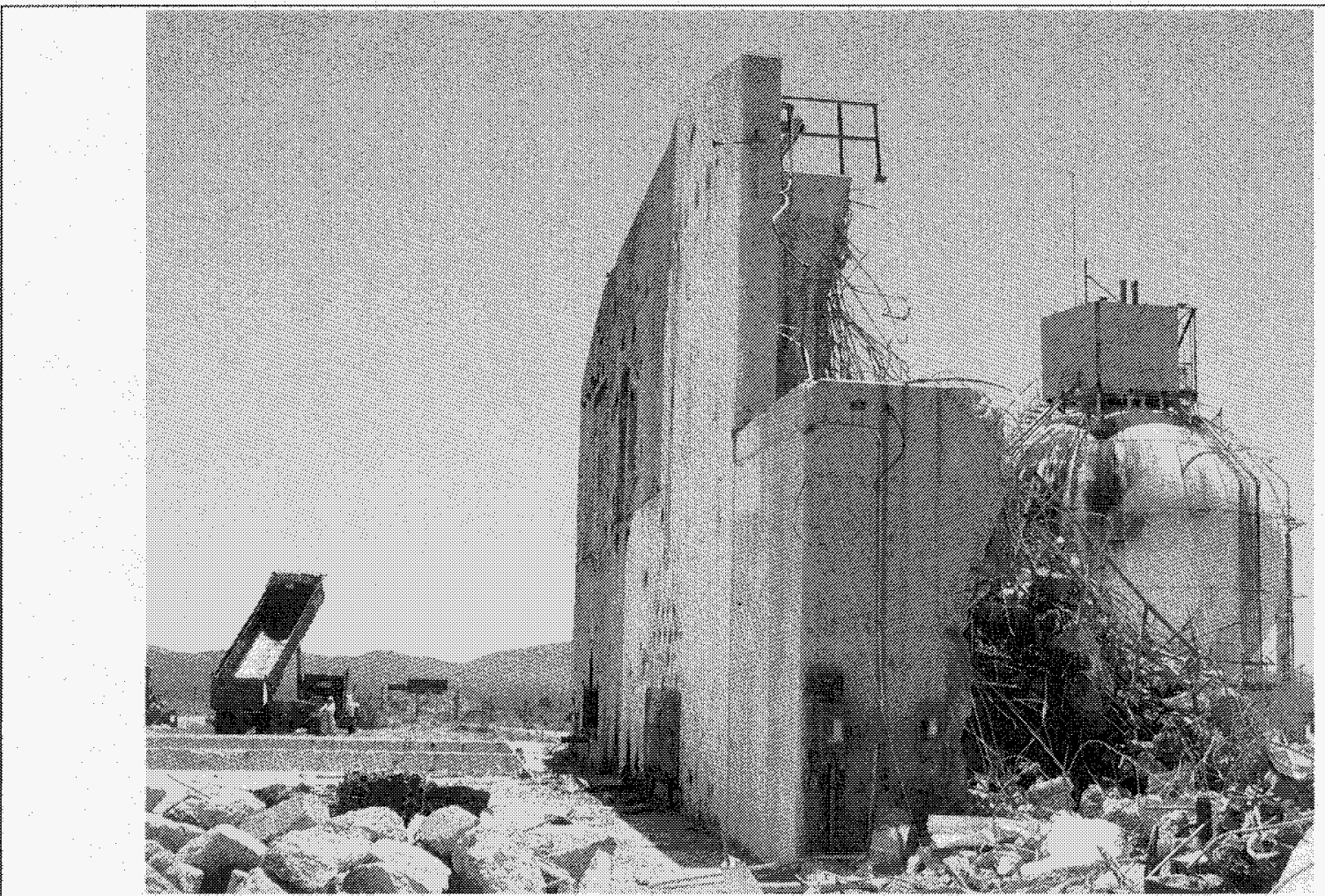

Photograph 21: Shield Wall After Demolition of Building 3113, Facing South, 06/15/2005

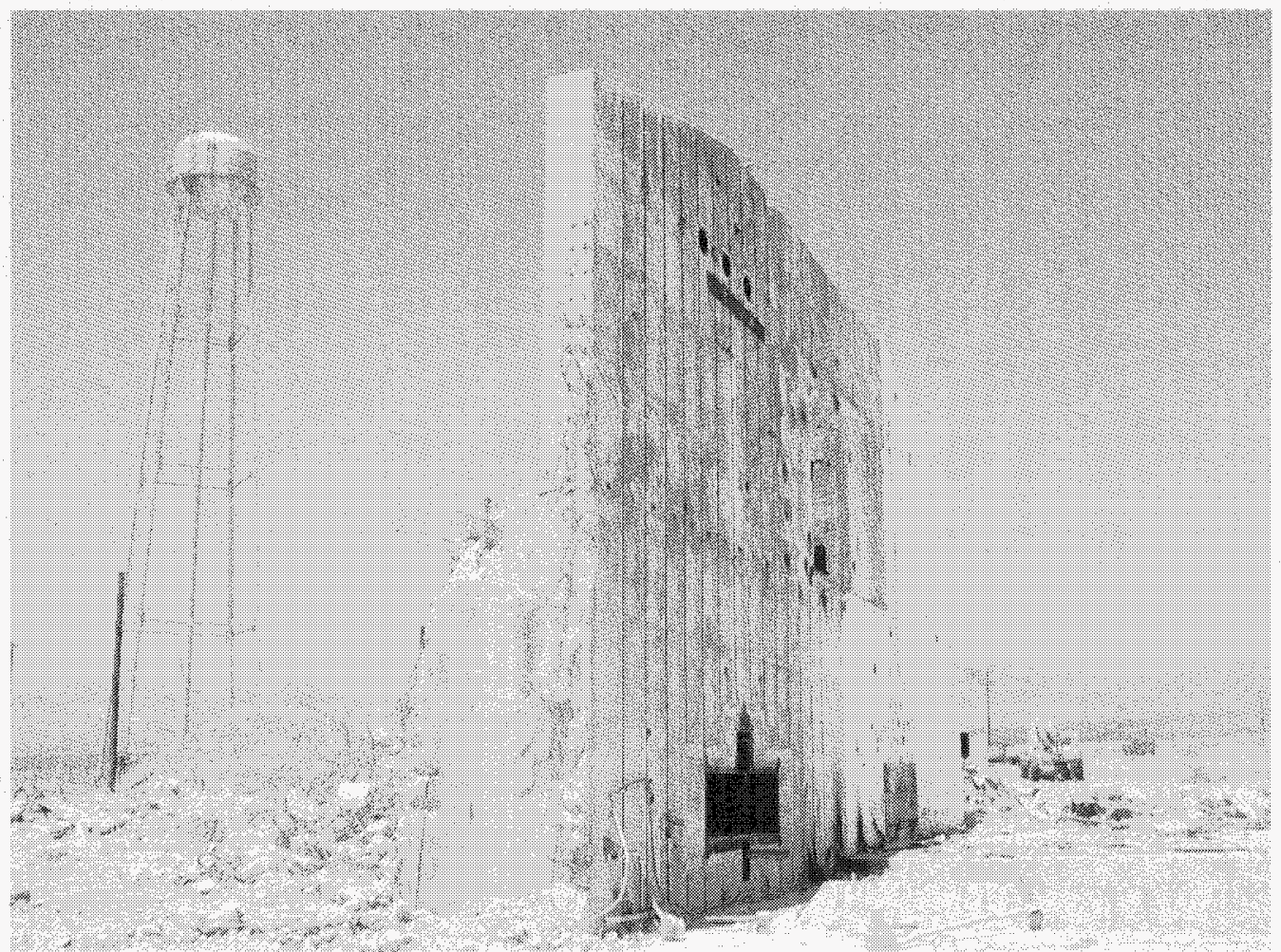

Photograph 22: Shield Wall After Demolition of Building 3113, Facing Northwest, 06/15/2005 


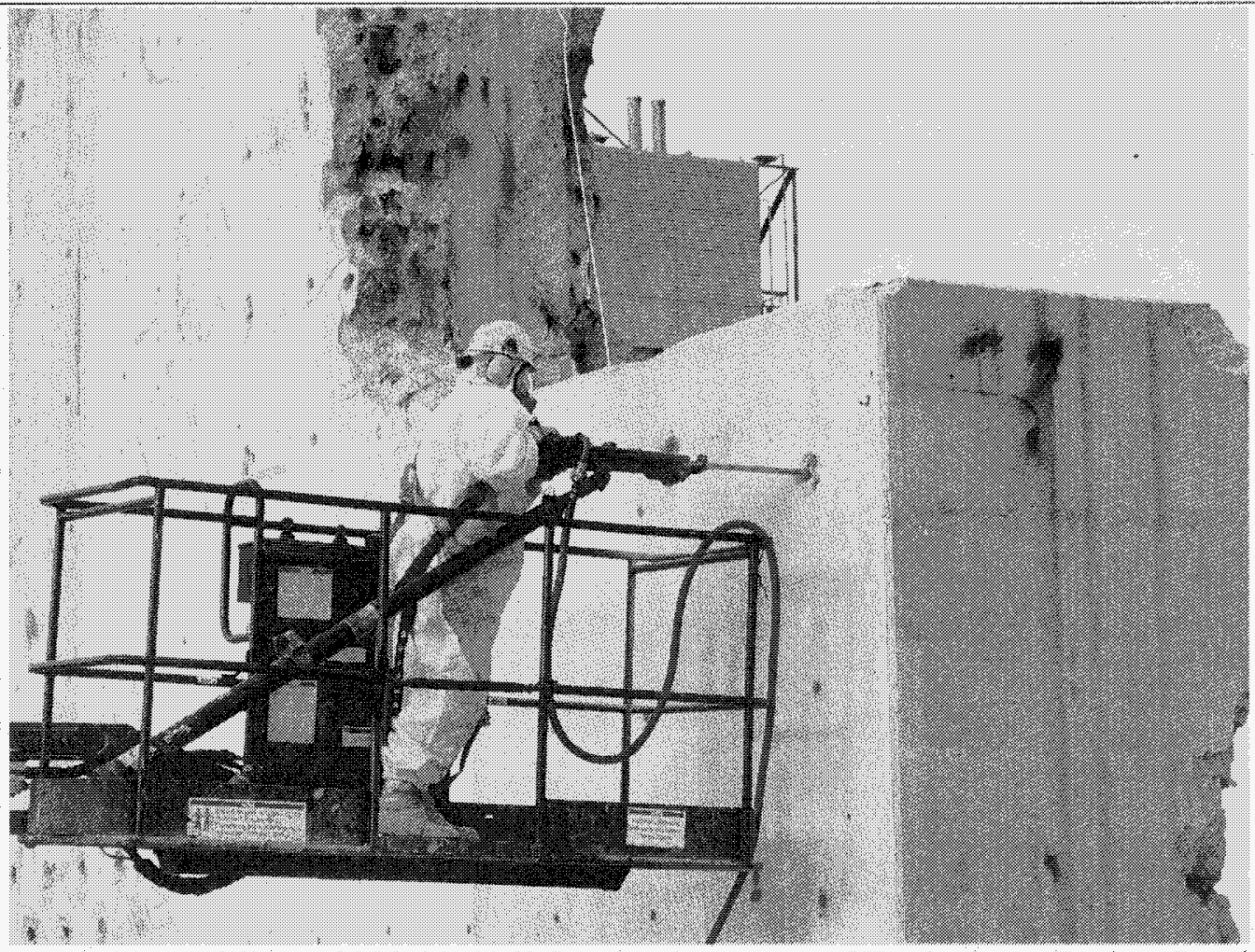

Photograph 23: Drilling of Shield Wall for Emplacement of Explosives, Facing Southwest, $06 / 21 / 2005$

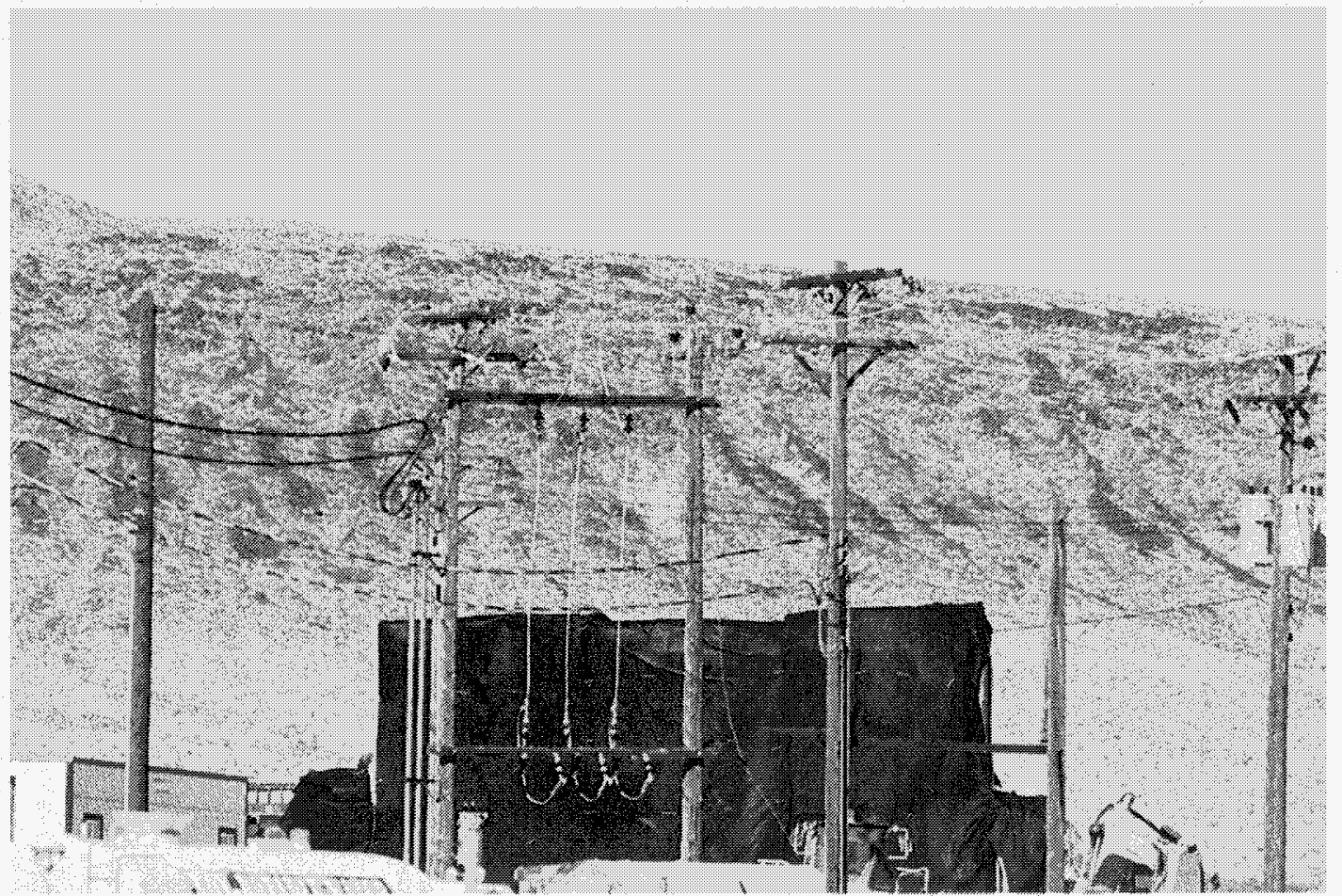

Photograph 24: Shield Wall Prepared for Explosive Demolition, Facing West, 06/23/2005 


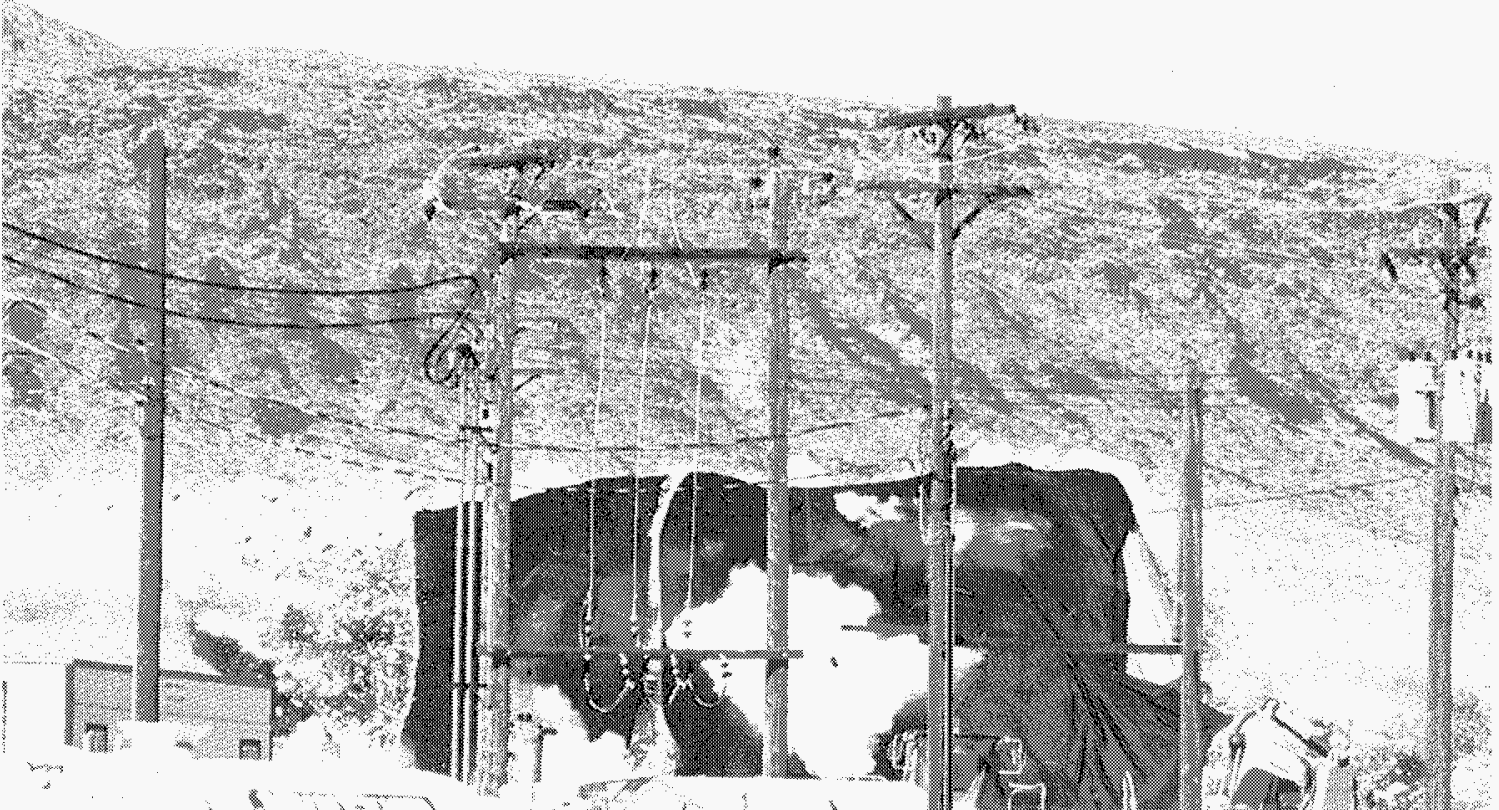

Photograph 25: Explosive Demolition of Shield Wall, Facing West, 06/23/2005

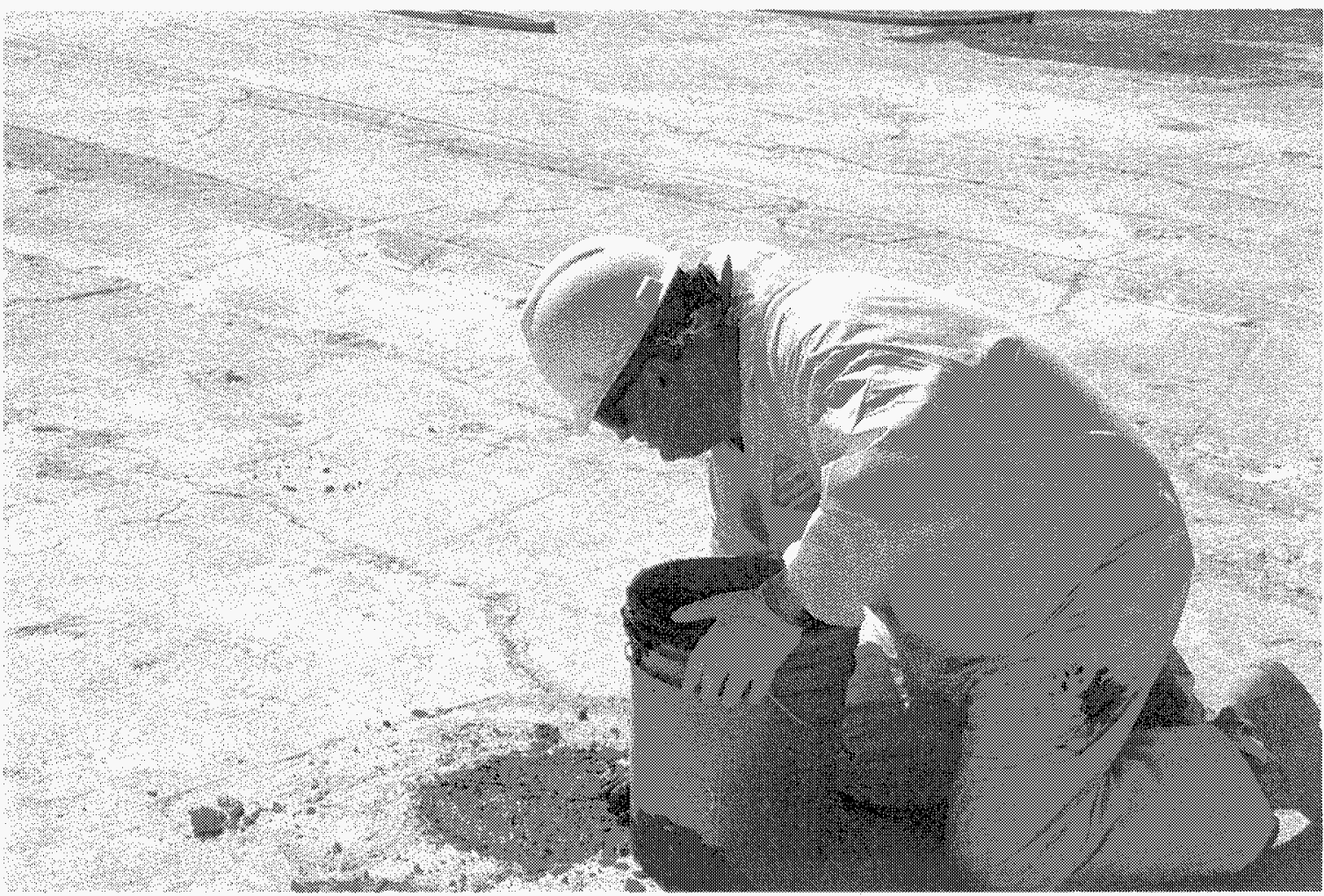

Photograph 26: Grouting of Penetrations, $06 / 29 / 2005$ 


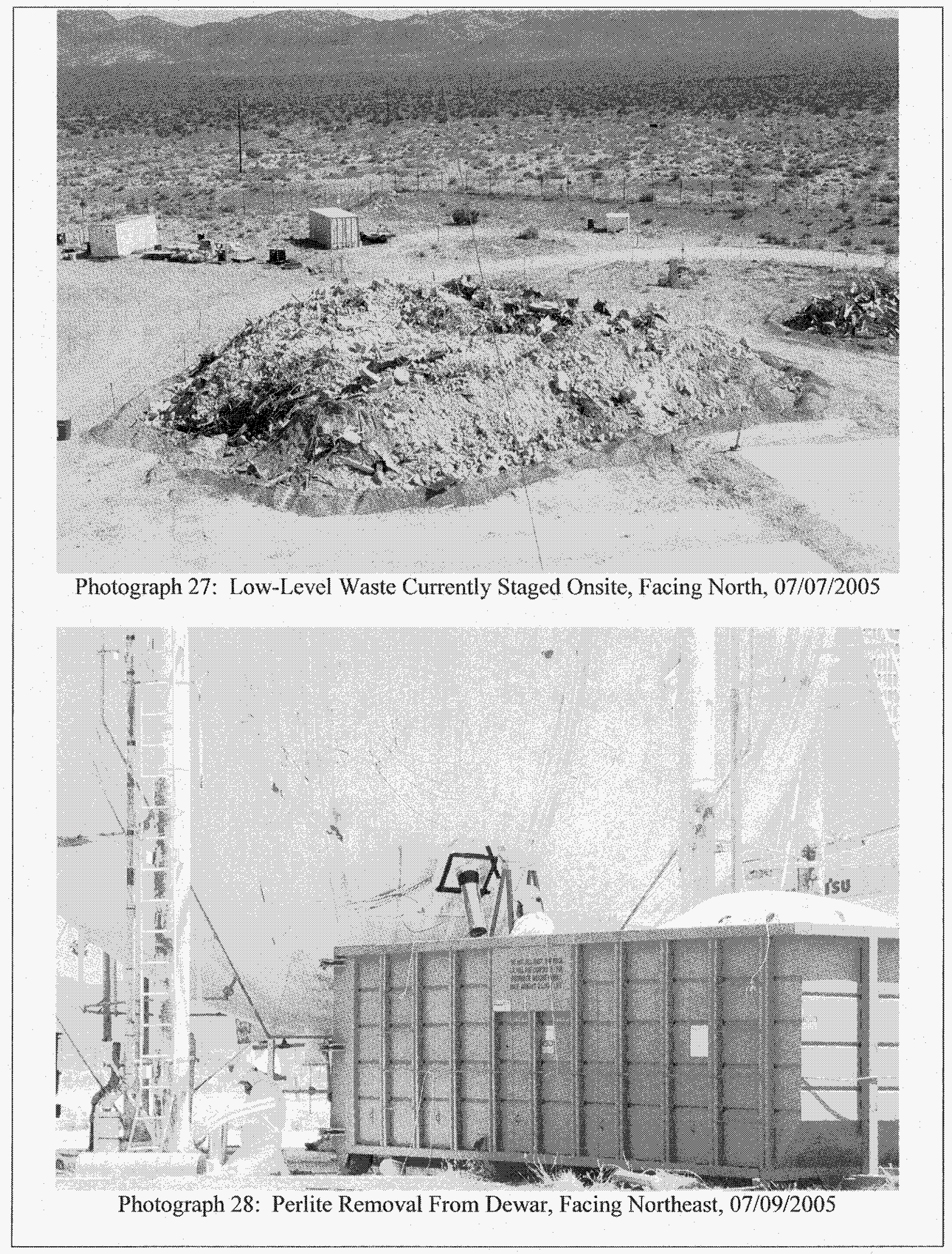




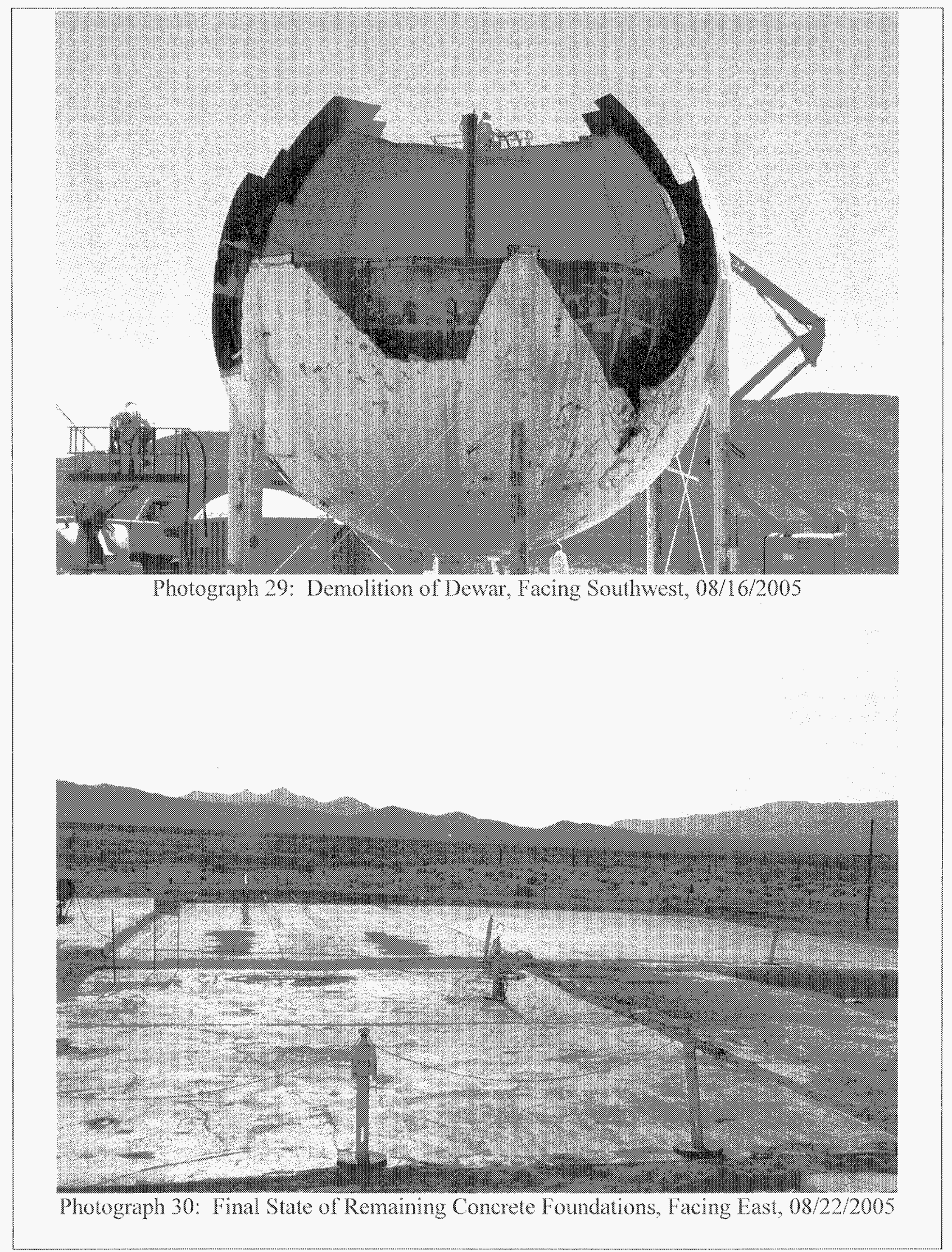




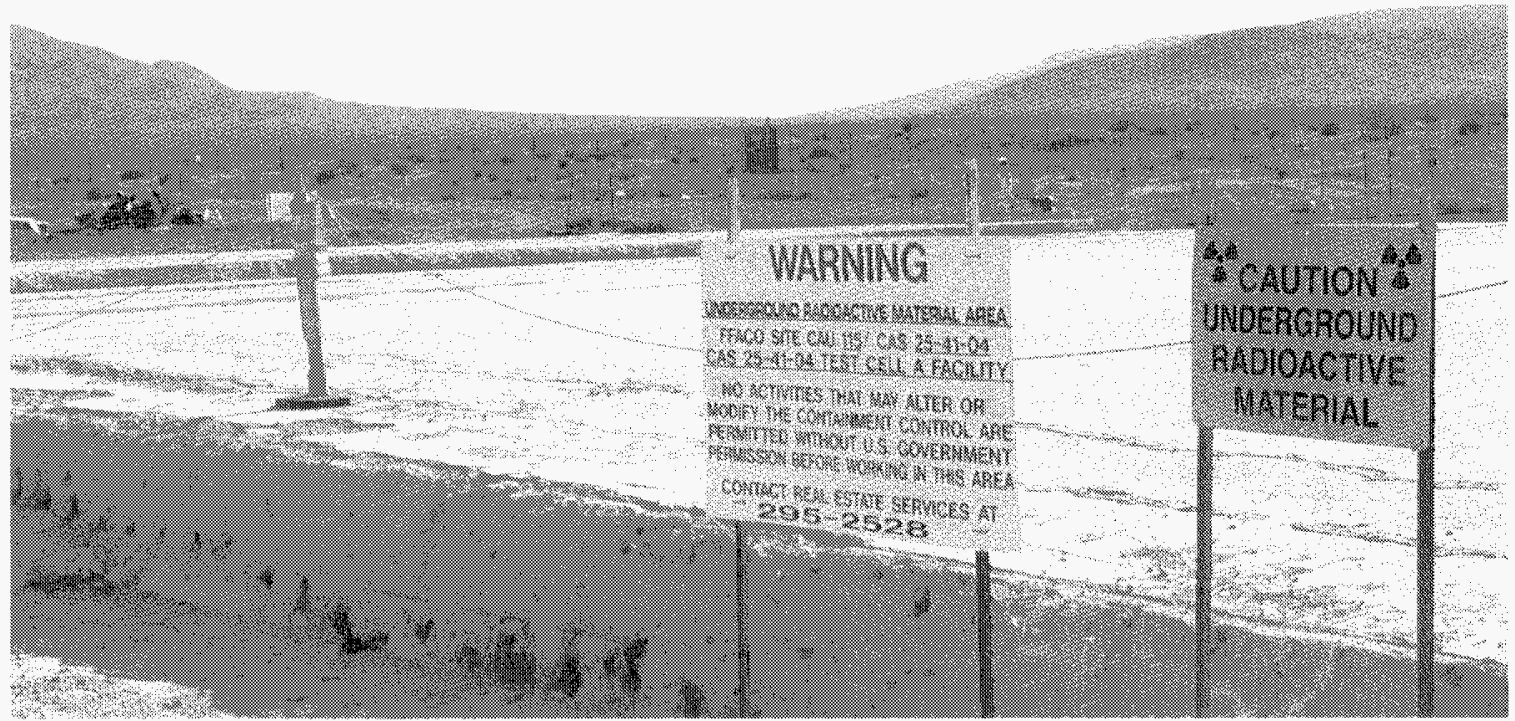

Photograph 31: Radiological Posting and Use Restriction Warning Sign for Reactor Pad, Facing Southeast, 08/22/2005 


\section{APPENDIX G}

\section{RECORD OF TECHNICAL CHANGE TO THE SAFER PLAN}


Closure Report - CAU 115

Section: Appendix G

Revision: 0

Date: March 2006

THIS PAGE INTENTIONALLY LEFT BLANK 


\section{RECORD OF TECHNICAL CHANGE}

Technical Change No. SAFER-1

Project/Job No. CAU 115

Page $\frac{1}{1}$ of

of $\quad 8$

Date $4 / 15 / 05$

Project/Job Name Corrective Action Unit 115 Test Cell A Facility SAFER Plan

The following technical changes (including justification) are requested by:

Michael Kruzic

(Name)

Bechtel Nevada Task Manager

(Title)

Description of Change:

1. Page xi, $4^{\text {th }}$ paragraph. Replace the third and fourth sentences with the following:

Removal of the dewar and concrete structures south and west of Building $3113 \mathrm{~A}$ are not part of the CAU 115 closure activities, but may be removed as a best management practice. Closure of CAU 115 will be accomplished by completing the following activities:

Justification - This change is needed because the dewar and liquid hydrogen station may be removed as a best management practice. Clean closure will not be obtained. The Radiological Controls Department has determined that the concrete reactor pad, and potentially the soil under portions of the pad, has been radiologically activated. This decision was based on ISOCS analysis conducted on the reactor pad. The ISOCS surveys showed activation products that exceed the criteria for a radiological materials area. The activation products included europium-152 and - 154 with maximum concentrations of 21 and 6.4 picocuries per gram ( $\mathrm{pCi} / \mathrm{g}$ ), respectively, and cobalt 60 with a maximum concentration of $10.3 \mathrm{pCi} / \mathrm{g}$. Because of this activation, the reactor pad will need to be posted as an underground radioactive material area (URMA) to warn of radiation associated with the matrix of the concrete and potentially activated soil beneath the thin portion of the pad.

2. Page xi, $7^{\text {th }}$ bullet. Replace the seventh bullet with the following sentence:

Fill all trenches and large cracks on the reactor pad with grout or similar material.

Justification - The newly proposed closure methods for the reactor pad include grouting all of the trenches present on the reactor pad, rather than removing the fixed contamination present on the interior surface. This includes the soil and rail ties located on the eastern portion of the reactor pad and the concrete bottoms of the remainder of the rail trenches and two large trenches. This will cover and shield the fixed contamination located in the trenches, reduce worker exposure to radionuclides during closure activities, and shorten the duration of decontamination activities. There is additional clarification on the change in decontamination activities in Change 26 of this ROTC. Because the reactor pad will already be posted as a URMA from the activation of the concrete and potentially activated soil beneath it, covering the fixed contamination with grout will not change the final radiological posting. The radiological posting will be necessary unless all of the activated concrete and any activated soil beneath it are removed.

3. Page xi, $8^{\text {th }}$ bullet. Delete the second sentence: Dewar not included in the closure of CAU 115.

Justification - This change is needed because the dewar and liquid hydrogen station may be removed as a best management practice. In addition, it has already been mentioned that the dewar may be removed as an option to the demolition subcontract in Change 1 to this ROTC. 
4. Page xi. $9^{\text {th }}$ bullet. Replace the sentence with the following:

Perform final radiological surveys to establish proper controls of concrete foundations and reactor concrete pad.

Justification - Final radiological surveys will be conducted in the same method as originally planned, but the reactor pad will not be free released. The concrete foundations will still be surveyed and decontaminated as necessary to be free released. The radiological surveys are discussed further in Change 27 of this ROTC. After reviewing ISOCS results with the Radiological Controls Department, it has been determined that the reactor pad will not be able to be free released as originally indicated in the SAFER Plan. It has been determined that the pad has been activated from tests conducted on the reactor pad. The ISOCS surveys showed activation products that exceed the criteria for a radiological materials area. The activation products included europium-152 and -154 with maximum concentrations of 21 and 6.4 picocuries per gram (pCi/g), respectively, and cobalt-60 with a maximum concentration of $10.3 \mathrm{pCi} / \mathrm{g}$. This has caused the concrete and potentially the soil under the 6" portions of the concrete pad to become radioactive. This includes the surface and the interior composition of the concrete/soil. Because of this, it will not be possible to remove the fixed contamination from the surface of the pad as originally planned. Therefore, unless the entire pad and any activated soil underneath it is removed, it will be radiologically posted. Additional information on radiological posting is located in Change 26 of this ROTC.

5. Page 1, Section 1.0, $1^{\text {st }}$ paragraph. Replace the first sentence with the following:

This Streamlined Approach for Environmental Restoration (SAFER) Plan identifies the activities required for the closure of Corrective Action Unit (CAU) 115, Area 25 Test Cell A (TCA) Facility.

Justification - The proposed changes to the SAFER Plan will require radiological postings around the perimeter of the concrete reactor pad. With this change, this CAU would no longer be considered clean closed. Because of the radiological hazard present within the matrix of the concrete, a use restriction will need to be implemented to prevent intrusive activities. Closure in place with administrative controls will be the required closure alternative because of the radiological posting and use restriction.

\section{Page 2, Section 1.2. Replace the first sentence with the following:}

Closure of CAU 115 will be accomplished by completing the following activities:

Justification - As indicated in Change 5, the proposed changes to the SAFER Plan will require radiological postings around the perimeter of the concrete reactor pad. Because of the radiological hazard present within the matrix of the concrete, a use restriction will need to be implemented to prevent intrusive activities. Closure in place with administrative controls will be the required closure alternative since the site will require radiological posting and a use restriction.

7. Page 2, Section 1.2. Replace the third bullet with the following sentence:

Fill all trenches and large cracks on the reactor pad with grout or similar material.

Justification - The newly proposed closure methods for the reactor pad include grouting all of the trenches present on the reactor pad, rather than removing the fixed contamination present on the interior surface. This includes the soil and rail ties located on the eastern portion of the reactor pad and the concrete bottoms of the remainder of the rail trenches and two large trenches. This will cover and shield the fixed contamination located in the trenches, reduce worker exposure to radionuclides during closure activities, and shorten the duration of decontamination activities. Additional clarification on the change in decontamination activities in Change 26 of this ROTC. Because the reactor pad will already be posted as a URMA from the activation of the concrete and potentially activated soil beneath it, covering the fixed contamination with grout will not change the final radiological posting. 


\section{Page 2, Section $1.2,4^{\text {th }}$ bullet. Replace the second sentence with the following:}

Removal of the dewar and concrete structures south and west of Building 3113A are not part of the CAU 115 closure activities, but they may be removed as a best management practice.

Justification - This change is needed because the dewar has been added as an option to the demolition subcontract, but its removal is not included in the fee milestone.

9. Page 2, Section 1.2. Replace the fifth bullet with the following sentence:

Perform final radiological surveys to establish proper controls of concrete foundations and reactor concrete pad.

Justification - Final radiological surveys will be conducted by the same method as originally planned, but the reactor pad will not be free released. The concrete foundations will still be surveyed and decontaminated as necessary to be free released. The radiological surveys are discussed further in Change 27 of this ROTC. After reviewing ISOCS results with the Radiological Controls Department, it has been determined that the reactor pad will not be able to be free released as originally indicated in the SAFER Plan. It has been determined that the pad has been activated from tests conducted on the reactor pad. This has caused the concrete and potentially the soil under the 6" portions of the concrete pad to become radioactive. This includes the surface and the interior composition of the concrete/soil. Because of this, it will not be possible to remove the fixed contamination from the surface of the pad as originally planned. Therefore, unless the entire pad and any activated soil underneath it is removed, it will be radiologically posted. Additional information on radiological posting is located in Change 26 of this ROTC.

\section{Page 2, Section $1.2,2^{\text {nd }}$ paragraph. Change item 4 "provides for clean closure, and" to read as follows:}

4) downgrades the site's radiological controls

Justification - The site will not be able to be clean closed, as described in Change 5 to this ROTC, however the radiological controls currently present at the site will be downgraded. Currently the reactor pad is posted as a Contamination Area. After the trenches and cracks are filled with grout, the soil from the NW corner of the pad is removed, and all removable contamination greater than levels listed in Table 4-2 of the NV/YMP Radiological Control Manual is removed from the surface of the reactor pad, the reactor pad can be downgraded to a URMA, as described in Change 26 of this ROTC.

\section{Page 2, Section $1.2,2^{\text {nd }}$ paragraph. Change item 5 to read as follows:}

(5) reduces long-term surveillance and maintenance costs.

Justification - Closure activities at Test Cell A, including demolition of the facility and reducing radiological controls, will reduce costs required to conduct yearly surveillance and maintenance activities. This includes radiological, structural, and hantavirus surveys and corrective activities. Once the facility is brought to slab-on grade conditions, only yearly radiological surveys will be required to ensure that use restriction signs and radiological postings remain in good condition. Therefore, closure activities will reduce the long-term surveillance and maintenance costs but not entirely eliminate them.

\section{Page 2. Section $1.2,3^{\text {rd }}$ paragraph. Replace the last two sentences with the following:}

Radiologically controlled areas outside of CAU boundaries do not require use restrictions. CAU 115: Area 25

TCA Facility will be demolished to slab-on-grade and closed with administrative controls. A use restriction will be imposed for the footprint of the concrete reactor pad.

Justification - The first sentence was modified to clarify that the soil posted around the facility, currently an RMA, will not require a use restriction after closure, since this area is not part of CAU 115. The second sentence was changed because of the radiological hazard present within the matrix of the concrete, the underground drain line under the pad, and the potentially activated soil under the thinner portions of the reactor pad. Closure in place with administrative controls will 
be the required closure alternative since the concrete reactor pad will require radiological posting and use restriction. The use restriction will be implemented to prevent intrusive activities on the pad.

13. Page 2 Section $1.3,3^{\text {td }}$ bullet. Replace the sentence with the following:

Fill all trenches and large cracks on the reactor pad with grout or similar material.

Justification - The proposed closure methods for the reactor pad include grouting all of the trenches present on the reactor pad rather than removing the fixed contamination present on the interior surface. This includes the soil and rail ties located on the eastern portion of the reactor pad and the concrete bottoms of the remainder of the rail trenches and two large trenches. This will cover and shield the fixed contamination located in the trenches, reduce worker exposure to radionuclides during closure activities, and shorten the duration of decontamination activities. Additional clarification on the change in decontamination activities in Change 26 of this ROTC. Because the reactor pad will already be posted as a URMA from the activation of the concrete and potentially activated soil beneath it, covering the fixed contamination with grout will not change the final radiological posting.

\section{Page 3, Section $1.3 .5^{\text {th }}$ bullet. Replace the sentence with the following:}

Performance of final radiological surveys to establish proper controls of concrete foundation and pads.

Justification - Final radiological surveys will be conducted in the same method as originally planned, but the reactor pad will not be free released. The radiological surveys are discussed further in Change 27 of this ROTC. After reviewing ISOCS results with the Radiological Controls Department, it has been determined that the reactor pad will not be able to be free released as originally indicated in the SAFER Plan. It has been determined that the pad has been activated from tests conducted on the reactor pad. The activation products identified during ISOCS analysis included europium-152 and -154 with maximum concentrations of 21 and 6.4 picocuries per gram $(\mathrm{pCi} / \mathrm{g})$, respectively and cobalt- 60 with a maximum concentration of $10.3 \mathrm{pCi} / \mathrm{g}$. This has caused the concrete and potentially the soil under the 6" portions of the concrete pad to become radioactive. This includes the surface and the interior composition of the concrete/soil. Because of this, it will not be possible to remove the fixed contamination from the surface of the pad as originally planned. Therefore, unless the entire pad and any activated soil underneath it is removed, it will be radiologically posted. Additional information on radiological posting is located in Change 26 - Section 3.2.8 of this ROTC.

15. Page 3, Section 1.4. Replace the first sentence with the following:

This SAFER Plan has been developed to support the closure of CAU 115 as required by the FFACO, DQOs, and Project Organization (presented in Appendices A.1 and A.2).

Justification - The proposed changes to the SAFER Plan differ from those originally presented in DQOs. CAU 115 will not be clean closed, rather closed in place with administrative controls. Closure in place with administrative controls will be required because the site will be radiologically posted around the perimeter of the concrete reactor pad. In addition, a use restriction will be required to prevent intrusive activities from being conducted on the pads.

16. Page 4, Figure 1. Replace the box following hold point \#3, "Decontaminate radiologically contaminated pad to DOE 05400.5" with the following:

Fill all trenches and large cracks on reactor pad with grout or similar material.

Justification - As described in Change 7 of this ROTC, decontamination activities on the reactor pad will not be conducted to remove fixed surface contamination. Because the matrix of the concrete is activated, it will not be possible to remove the contamination without removing the entire concrete pad and any potentially activated soil beneath it. Therefore, if the trenches are filled with grout, covering the fixed contamination present at the bottom, the final pad posting will be the same. In addition, the grout will cover and shield the fixed contamination located in the trenches, reduce worker exposure to radionuclides during closure activities, and shorten the duration of decontamination activities. 
17. Page 12. Section 2.3.1.1, last paragraph. Replace the last sentence with the following:

Details on decontamination of the reactor pad are discussed in Section 3.2.8.

Justification - Change number 26 of this ROTC adds detail into Section 3.2.8 of the SAFER Plan to identify how and where the proposed decontamination activities will take place. This change is necessary since the only planned decontamination activities on the concrete reactor pad will be conducted after demolition activities, as described in Change 26 - in Section 3.2 .8 of this ROTC.

18. Page 15, Section $2.4,2^{\text {nd }}$ paragraph Replace the second sentence with the following:

Removal of the dewar and concrete structures south and west of Building 3113A are not part of the CAU 115 closure activities, but they may be removed as a best management practice.

Justification - This change is needed because the dewar has been added as an option to the demolition subcontract, but its removal is not included in the fee milestone.

19. Page 15, Section 2.4.1 Replace the first two paragraphs with the following:

Prior to demolishing the facility, all of the trenches and large cracks on the reactor concrete pad will be grouted or filled with similar material to minimize personnel exposure to fixed contamination within the trenches. After demolition of the moveable shed, Building 3130, additional grouting of two trenches partially covered by the shed will be conducted. After grouting and demolition activities are complete, the reactor concrete pad (Figure 11) and concrete foundation will be radiologically surveyed. Post-demolition surveys of the remaining concrete foundations and pads will be conducted as described in Section 3.2.9.

Justification - This section has been modified from the original SAFER Plan because it has been proposed to grout the fixed contamination present in the bottom of the trenches, rather than chipping these portions out. Reasons for this proposed method are detailed in Change 2 of this ROTC.

20. Page 17, Section $2.4 .2,2^{\text {nd }}$ paragraph. Delete the first two sentences from the paragraph to read as follows: Below-grade piping under the foundation of Buildings $3113,3113 \mathrm{~A}$, and $3113 \mathrm{~B}$, the concrete reactor pad, or below- or above-ground piping beyond the boundaries of this $\mathrm{CAU}$, will not be addressed in the closure of CAU 115.

Justification - This paragraph has been modified to concur with the proposed modification of the closure activities on the reactor pad. Because the concrete reactor pad has been activated, it will remain radiologically posted after closure activities. The removal of the underground pipeline will not affect this posting. By not removing the drain pipeline under the reactor concrete pad, there is less potential for personnel to be exposed to unknown quantities of radionuclides present within the pipe. Also the amount of time spent conducting removal activities on the reactor pad and the quantity of LLW generated will be reduced.

21. Page 19, Section 3.0. Replace the third paragraph with the following:

Closure of CAU 115 will be accomplished by removal of hazardous or regulated materials, demolition and disposal of the above-grade structures (Buildings $3113,3113 \mathrm{~A}, 3113 \mathrm{~B}$ ), filling of all trenches and large cracks on the reactor pad with grout or similar material, and decontamination of remaining concrete foundations, if necessary. Building 3130, the Movable Shed, will also be demolished as part of the closure. Removal of the dewar and concrete structures south and west of Building 3113A are not part of the CAU 115 closure activities, but they may be removed as a best management practice.

Justification - As described in Change 5 of this ROTC, CAU 115 will not be able to be clean closed, but rather closed in 
place with administrative controls due to the reactor concrete pad. This paragraph was modified to remove the word "clean closure" for this reason. In addition, grouting of the trenches on the reactor pad was added to the list of closure activities. This activity was added because grouting activities on the reactor pad is a necessary step to reach the closure alternative proposed in this ROTC. This involves covering the fixed contamination in the bottom of the trenches with grout to keep personnel and debris from demolition activities from entering. In addition, the last sentence regarding the dewar and concrete structures south and west of B. $3113 \mathrm{~A}$ was modified to allow their removal during demolition activities as a best management process if funding and manpower are available.

22. Page 24. Section 3.2. Replace the third bullet with the following sentence:

Fill all trenches and large cracks on the reactor pad with grout or similar material.

Justification - The newly proposed closure methods for the reactor pad include grouting all of the trenches present on the reactor pad rather than removing the fixed contamination present on the interior surface. This includes the soil and rail ties located on the eastern portion of the reactor pad and the concrete bottoms of the remainder of the rail trenches and two large trenches. This will cover and shield the fixed contamination located in the trenches, reduce worker exposure to radionuclides during closure activities, and shorten the duration of decontamination activities. Additional clarification on the change in decontamination activities is given in Change 26 of this ROTC. Because the reactor pad will already be posted as a URMA from the activation of the concrete and potentially activated soil beneath it, covering the fixed contamination with grout will not change the final radiological posting.

23. Page 24 , Section $3.2,4^{\text {th }}$ bullet. Delete the second sentence: Dewar not included in the closure of CAU 115.

Justification - This change is necessary to clarify that the dewar has been added as an option to the demolition subcontract, but its removal is not included in the fee milestone. If funding and manpower are available, this option may be pursued.

24. Page 24, Section 3.2. Replace the fifth bullet with the following:

Perform final radiological surveys to establish proper controls of concrete foundation and reactor pad.

Justification - Final radiological surveys will be conducted by the same method as originally planned, but the reactor pad will not be free released. The concrete foundations will still be surveyed and decontaminated as necessary to be free released. The radiological surveys are discussed further in Change 27 of this ROTC. Additional detail on why the reactor pad will not be free released is discussed in Change 4 to this ROTC.

25. Page 26. Section 3.2.5. Delete the last paragraph of this section.

Justification - The deleted paragraph discussed the removal of the piping from the concrete reactor pad. Because the reactor pad has been activated, it will remain radiologically posted after closure activities; therefore removal of the underground pipeline will not affect this posting. By not removing the pipeline, there is less potential for personnel to be exposed to unknown quantities of radionuclides present in the pipe. Also the amount of time spent conducting activities on the reactor pad and the quantity of LLW generated will be reduced.

26. Page 28. Section 3.2.8. Replace the first paragraph with the following:

Radiological contamination will be removed primarily by scabbling of concrete surfaces, removal of concrete with either hand tools or heavy equipment, or by wiping or vacuuming methods. All concrete surfaces left after demolition activities will be surveyed as described in Section 3.2.9.

ISOCS analysis conducted at nine locations on the reactor pad has shown that the concrete has been radiologically activated. ISOCS analysis detected activation products, including europium- 152 and -154 and cobalt- 60 , which exceed the criteria for a radiological materials area. Concrete activation is where the matrix of the concrete, rather than just the surface, has become radioactive from neutrons passing into it during reactor test activities. Because of this activation, hand-held radiological survey instruments indicate contamination levels above those listed in Table 1 for fixed contamination. This radiation cannot be removed by chipping off the surface layer of concrete, because the entire pad down to soil is expected to be activated, if similar to the activation depth seen in 
core samples from the shield wall. However, any areas with removable contamination detected above Table 1 limits, from swipe surveys, will be decontaminated. Fixed contamination is present in the form of soil and hot particles deposited in the base of the large trenches, rail trenches, and within porous railroad ties on the east side of the reactor pad. These areas of the concrete reactor pad will be filled/covered with grout or similar material to minimize the amount of worker exposure to the elevated levels of radioactivity. The fixed contamination at the bottom of the trenches will remain in place and will not affect the final posting of the reactor pad. The final posting of the concrete reactor pad will be a URMA due to the activated concrete and potentially activated soil beneath the thinner portions of the pad.

Justification - This section has been added to the work package to detail the proposed changes to the decontamination of the reactor pad trenches. As indicated in the change above, the pad will remain a URMA after closure, and the removal of the fixed contamination in the rails will not affect the site closure of the pad. Once this was determined, the risk associated with removing the fixed contamination from the rail trenches was re-evaluated. The above change has been proposed to reduce the amount of potential exposure to the workers from high levels of fixed contamination in the rail trenches and rail ties located on the east side of the reactor pad. In addition to reducing worker exposure, the amount of low-level waste generated from the pad will be significantly reduced, along with the number of days spent conducting decontamination activities and packaging waste in this area.

27. Page 28, Section $3.2 .9,1^{\text {st }}$ paragraph. Replace second and third sentences with the following:

Surveys will be completed using survey instruments and methods described in Section 3.2.7.1 and Section 3.2.7.2. If surveys indicate the presence of radiological contamination, excluding fixed contamination on the reactor pad, decontamination and subsequent verification surveys will be completed.

Justification - The referenced Sections in the first sentence have been changed since the Section numbers in the SAFER Plan were incorrect. They have been changed to reference the correct sections. The second sentence was changed to match the changed decontamination activities on the reactor pad described in Change 26 of this ROTC.

28. Page 28 , Section $3.2 .9,3^{\text {rd }}$ paragraph. Replace the last sentence with the following:

Radiological signs will be placed on four sides around the reactor pad no further than $30.5 \mathrm{~m}$ (100 ft) apart.

Because the reactor pad will be posted as a URMA and is covered under CAU 115, a use restriction for this area will be required. Use restriction signs will be posted at the four corners of the reactor pad. Post-closure monitoring and posting requirements will be defined in the CR. Figure 14 shows the most likely posting scenario for the concrete reactor pad. Any changes to this configuration during closure activities will be documented in the $\mathrm{CR}$.

Justification - This sentence has been added to the last paragraph to provide detail on how the site will be posted after closure. This can be seen on the attached figure.

30. Page 29. Section 3.5. Replace the fifth bullet with the following:

Decontamination of the remaining concrete foundations and filling all trenches and large cracks on the reactor pad with grout or similar material.

Justification - The second half of the sentence was changed to include the change in closure activities on the concrete reactor pad. Removable contamination on the pad will be removed, but the fixed contamination present in the trenches will be covered by grout, as discussed in Change 26 to this ROTC.

31. Page 33. Section 5.2. Replace the first sentence with the following:

The waste streams that will be generated during closure of CAU 115 are discussed in the following sections.

Justification - The words "clean closure" have been removed from this sentence because the site will closed with administrative controls under the proposed changes to the SAFER Plan, as described in Change 5 - Section 1.0 of this ROTC. 
The project tine will be decreased by approximately 6 days.

Applicable Rroject-Specific Documont(s): Streanlined Apprasch for Envtronmental Rectoration Plan for Correcrive Action Unit 115: Aree 25 Test Coll A Pactisy, Neveda Tort Sie, Nevado, Rovision I

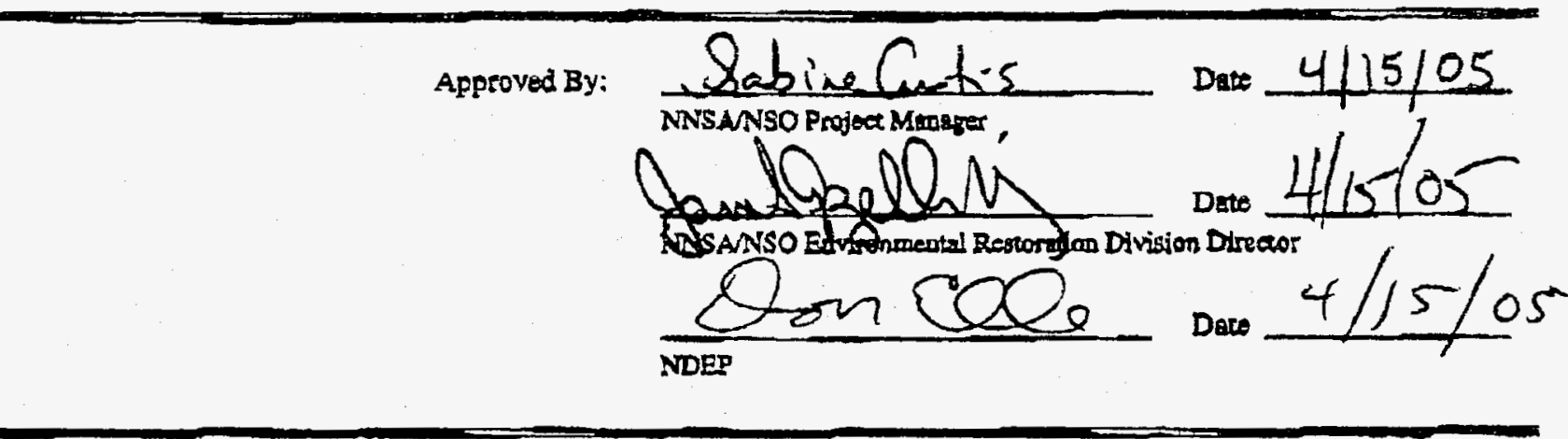




\section{LIBRARY DISTRIBUTION LIST}


Revision: 0

Date: March 2006

THIS PAGE INTENTIONALLY LEFT BLANK 


\section{LIBRARY DISTRIBUTION LIST}

U.S. Department of Energy

National Nuclear Security Administration

Nevada Site Office

Technical Library

P.O. Box 98518 , M/S 505

Las Vegas, NV 89193-8518

U.S. Department of Energy

Office of Scientific and Technical Information

P.O. Box 62

Oak Ridge, TN 37831-0062

Southern Nevada Public Reading Facility

c/o Nuclear Testing Archive

P.O. Box 98521, M/S 400

Las Vegas, NV 89193-8521

Manager, Northern Nevada FFACO

Public Reading Facility

c/o Nevada State Library \& Archives

Carson City, NV 89701-4285
1 (Uncontrolled)

1 (Uncontrolled, electronic copy)

2 (Uncontrolled, electronic copies)

1 (Uncontrolled, electronic copy) 
Closure Report - CAU 115

Section: Library Distribution List

Revision: 0

Date: March 2006

THIS PAGE INTENTIONALLY LEFT BLANK 